\title{
Le Bison des steppes, Bison priscus Bojanus, 1827, de la grotte d'Habarra à Arudy (Pyrénées-Atlantiques)
}

The Steppe Bison, Bison priscus Bojanus, 1827, from Habarra Cave, Arudy (Pyrénées-Atlantiques)

François Prat, Françoise Delpech, Nicolas Cancel, Jean-Luc Guadelli et René Slott-Moller

\section{OpenEdition}

\section{Journals}

Édition électronique

URL : http://journals.openedition.org/paleo/1362

DOI : $10.4000 /$ paleo.1362

ISSN : 2101-0420

\section{Éditeur}

SAMRA

\section{Édition imprimée}

Date de publication : 1 décembre 2003

Pagination : 1-102

ISSN : 1145-3370

\section{Référence électronique}

François Prat, Françoise Delpech, Nicolas Cancel, Jean-Luc Guadelli et René Slott-Moller, «Le Bison des steppes, Bison priscus Bojanus, 1827, de la grotte d'Habarra à Arudy (Pyrénées-Atlantiques) », PALEO [En ligne], 15 | 2003, mis en ligne le 04 août 2010, consulté le 07 juillet 2020. URL : http:// journals.openedition.org/paleo/1362 ; DOI : https://doi.org/10.4000/paleo.1362

Ce document a été généré automatiquement le 7 juillet 2020 .

\section{(c) (i) (9)}

PALEO est mis à disposition selon les termes de la licence Creative Commons Attribution - Pas d'Utilisation Commerciale - Pas de Modification 4.0 International. 


\section{Le Bison des steppes, Bison priscus Bojanus, 1827, de la grotte d'Habarra à Arudy (Pyrénées-Atlantiques)}

The Steppe Bison, Bison priscus Bojanus, 1827, from Habarra Cave, Arudy

(Pyrénées-Atlantiques)

François Prat, Françoise Delpech, Nicolas Cancel, Jean-Luc Guadelli et René Slott-Moller

\section{Les gisements d'Habarra}

Ce sont les membres du Groupe Spéléologique de la Vallée d'Ossau (G.S.V.O.) qui, les premiers, en 1984, observèrent les ossements affleurant au sommet du remplissage sédimentaire de la grotte. Aussitôt, ils firent part de leur découverte à E. de Valicourt du Groupe Archéologique des Pyrénées-Occidentales qui alerta Geneviève Marsan, alors correspondante de la Direction des Antiquités Préhistoriques d'Aquitaine et le regretté paléontologiste $\mathrm{A}$. Clot. Ces derniers se rendirent sur les lieux où ils constatèrent la présence de vestiges d'animaux, les uns d'âge holocène, les autres d'âge pléistocène. A. Clot identifia sur place plusieurs mammifères (Cerf, Renne, Bison, Bœuf, Ovicaprin, Cheval, Rhinocéros et Chien) représentés soit par des os isolés, soit par leur squelette presque entier ou très partiellement conservé. Ultérieurement il procéda à une première évaluation du nombre minimum d'individus de chaque espèce. Peu après, des fouilles de sauvetage furent entreprises par le Groupe Spéléologique de la Vallée d'Ossau, le Groupe Archéologique des Pyrénées-Occidentales, la Direction des Antiquités Préhistoriques d'Aquitaine (Dir. J.-Ph. Rigaud) et des chercheurs de l'Institut du Quaternaire de l'Université de Bordeaux I (Rigaud 1986). Les renseignements concernant les travaux effectués in situ et les premiers examens ont été consignés dans un rapport rédigé en 1985 par G. Marsan qui avait assuré la liaison des divers intervenants. Ce rapport est accompagné d'un inventaire par secteur des pièces 
déterminées et de relevés topographiques réalisés par A. Clot, E. de Valicourt et J. Coucouron.

2 La grotte d'Habarra, longue de 35 mètres environ, s'ouvre près du village d'Arudy dans le département des Pyrénées Atlantiques (fig. 1). Elle est orientée sud-est/nord-ouest suivant son axe principal. On y accède par un petit gouffre situé à une altitude de 770 mètres mais un deuxième puits perfore la voûte 20 mètres plus bas. Comme il est dit dans le rapport cité précédemment, ces deux ouvertures ont joué et, peut-être, jouent encore le rôle de piège naturel, ce qui explique la présence de nombreux restes de carcasses d'animaux d'âges divers dans le remplissage et sur le sol de la caverne. Il existe deux zones de forte concentration en ossements, elles-mêmes subdivisées en secteurs (fig. 2) :

- le gisement supérieur qui, dans sa partie actuellement connue, s'étend le long de la pente sur une dizaine de mètres environ de l'entrée amont jusqu'au deuxième puits ; il comprend les secteurs A, B, C, D, E, F, G et O ;

- le gisement inférieur déterminé par des découvertes effectuées sur quelques mètres en aval du deuxième puits ; secteurs I, J, K, L et M.

Figure 1 - Localisation géographique de la grotte d'Habarra. Figure 1 - The location of Habarra Cave.

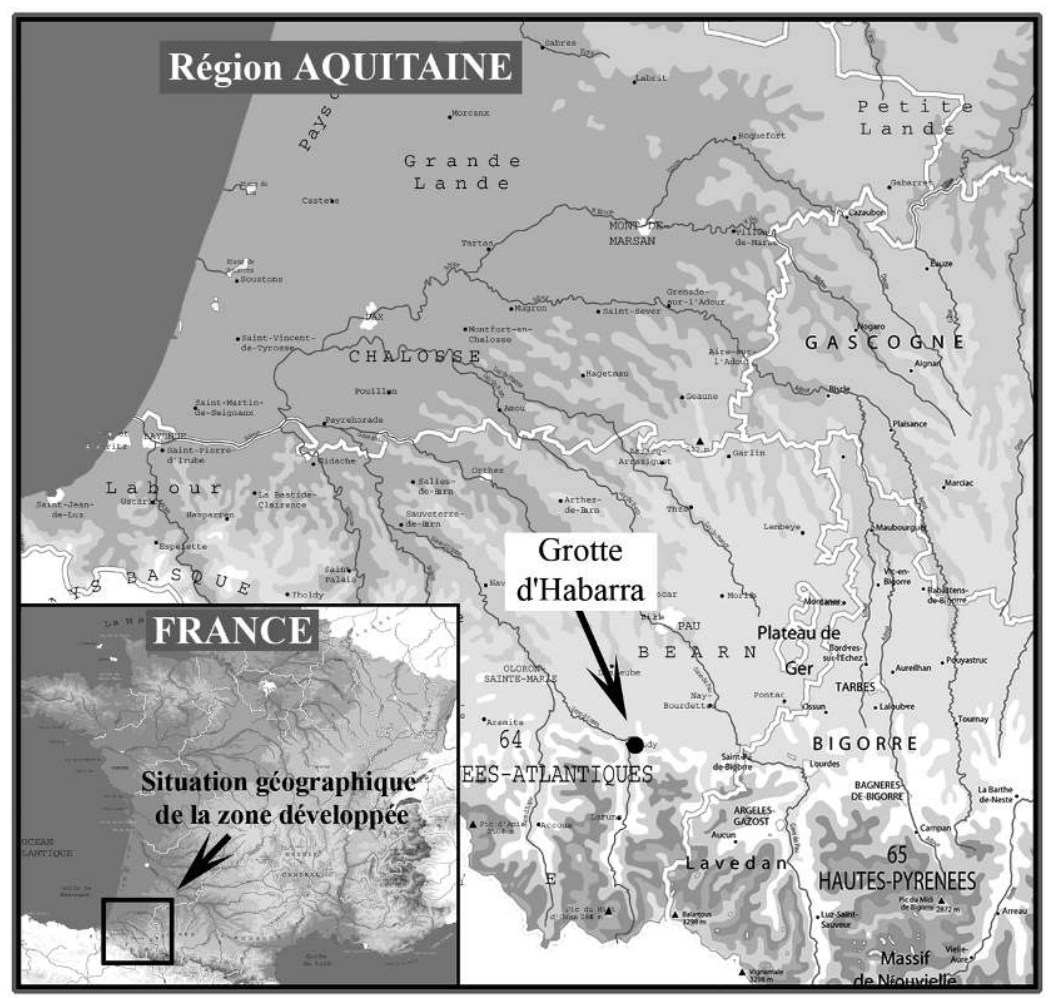


Figure 2 : Grotte d'Habarra, topographie d'après relevés de A. Clot, E. de Valicour et J. Coucouron (in Marsan 1985).

Figure 2: The topography of Habarra Cave.

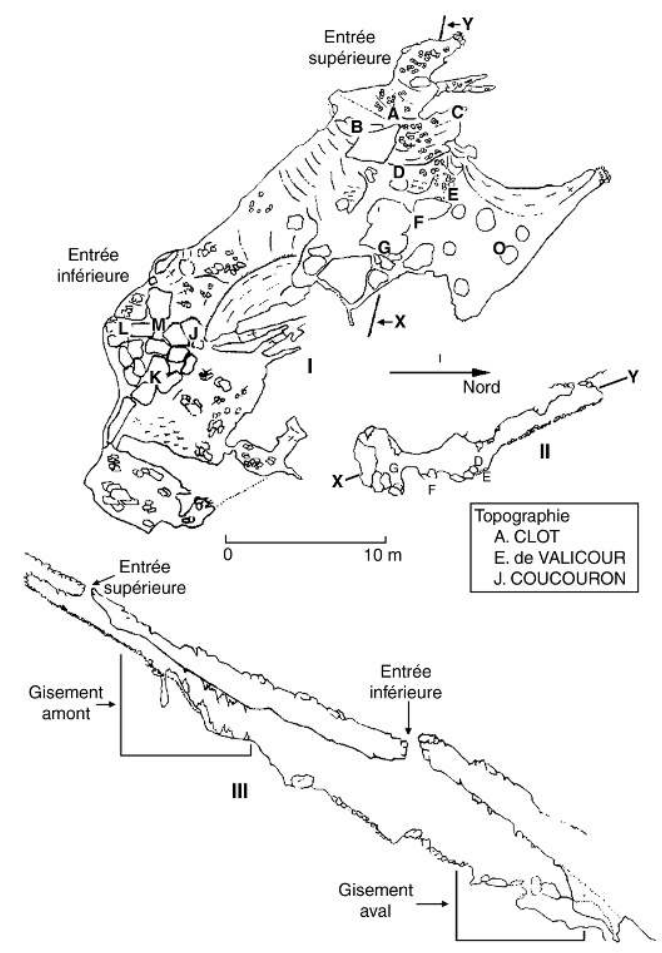

3 Des restes de chevaux, trouvés dans le secteur $\mathrm{K}$, ont été datés par la méthode $\mathrm{du}{ }^{14} \mathrm{C}$ de $28000 \pm 700$ ans BP (Gif-7105), le radius d'un bison du secteur E de $14500 \pm 260$ ans BP (Gif 7106) tandis que des ossements de Cerf du secteur L donnent un âge de $28200 \pm 700$ BP (Ly-4480) ${ }^{1}$.

4 Seules les pièces de ces gisements se rapportant à l'espèce Bison priscus Bojanus, 1827 ont retenu ici notre attention. Elles ont fait l'objet de comparaisons avec des documents appartenant à la même espèce mais de provenances autres ainsi qu'avec des os appartenant aux deux espèces actuelles : Bison bonasus Linnaeus, 1758, le Bison d'Europe et Bison bison Linnaeus, 1758, le Bison d'Amérique. A cette date aucun ossement de Bison $^{2}$ n'a été découvert dans les secteurs $\mathrm{G}, \mathrm{H}, \mathrm{O}, \mathrm{K}$ et $\mathrm{L}$.

\section{Identification des individus du genre Bison. Les données du terrain. Mise en connexion anatomique des pièces}

5 Le plan de répartition des ossements par locus effectué par les fouilleurs a, évidemment, beaucoup facilité le regroupement des pièces se rapportant au même individu. Les résultats les plus probants ont été obtenus à partir d'os des membres découverts dans les secteurs $\mathrm{A}, \mathrm{B}$ et $\mathrm{E}$ particulièrement riches et assez peu bouleversés après décomposition des cadavres. Des connexions anatomiques s'y trouvaient parfois presque conservées et, par chance, les bisons dont les os se mêlaient plus ou moins sur une aire réduite différaient sensiblement soit par la taille (bisons $n^{\circ} 1$ et $n^{\circ} 2$ ) soit par l'âge (bisons $n^{\circ} 4$ et $n^{\circ} 5$ ). En revanche, dans le secteur $D$, autre point de forte 
concentration, les vestiges de plusieurs sujets représentés chacun par quelques pièces seulement n'ont permis que de rares essais de mise en connexion.

Nous rapportons au même animal: 1 ) deux éléments contigus du squelette (ex. fémurtibia, tibia-talus) dont les dimensions s'harmonisent et qui s'ajustent parfaitement l'un à l'autre ; 2) deux os homologues (humérus droit-humérus gauche) dont les mesures respectives traduisent une taille et des proportions comparables et dont les détails anatomiques révèlent une symétrie satisfaisante. Sont aussi attribués au même sujet les os d'un membre antérieur et d'un membre postérieur quand les conditions de gisement (position dans le secteur), la compatibilité de leurs dimensions, leur aspect et leur état de fossilisation nous y invitent. Un exemple en est donné à propos du bison $n^{\circ} 1$. L'attribution de pièces du squelette axial (souvent incomplet et mal conservé), voire des scapulas, à un animal déterminé à partir des os des membres s'est révélée parfois malaisée. Il faut également rappeler que les fouilles pratiquées en 1984 avaient surtout pour but de recueillir les pièces les plus exposées aux prédateurs en tous genres, les chercheurs ayant bien noté que le remplissage de la caverne est fossilifère sur une forte épaisseur.

\section{Mensurations des pièces}

7 La prise en considération des dimensions des ossements a joué un rôle important dans l'identification des individus (évaluation de la stature, mise en évidence du caractère plus ou moins massif des formes, détermination du sexe). Nous indiquons sur les figures 3 à 8 la façon dont le crâne et les os des membres et des ceintures ont été mesurés. En général, elle rappelle celle conseillée par d'autres auteurs tels Von den Driesch (1976) et, pour les crânes, Mc Donald (1981). On notera que les saillies de la région proximale des humérus et fémurs se trouvant souvent endommagées, la longueur de ces os a été le plus souvent mesurée de l'extrémité distale au sommet de la tête articulaire. Nous avons fréquemment calculé, comme beaucoup d'autres, un indice de robustesse : c'est le rapport centésimal de la largeur ( souvent largeur minimum ou largeur au milieu) à la longueur de l'os considéré. Dans les tableaux, le numéro de chaque mensuration correspond à celui qu'elle porte sur la figure destinée à montrer comment l'os est mesuré. Cependant, pour éviter des retours en arrière nous avons cru utile d'y rappeler, en termes très abrégés, la façon dont la mesure a été prise.

Les squelettes de Bison d'Habarra devant être mis en comparaison avec ceux d'autres bisons actuels et fossiles, nous avons examiné et mesuré des documents conservés dans divers établissements. Nous remercions ici vivement les responsables de collections qui nous ont accueillis :

- Natural History Museum, London. Osteology (Mammal Section) : squelettes mesurés par N. Byrne. Dans nos tableaux, les éléments qui s'y rapportent présentent la mention B.M. suivie du numéro d'inventaire.

- Museum National d'Histoire Naturelle (Paris), Laboratoire d'Anatomie comparée. Les pièces examinées portent la mention M.P. suivie du numéro d'inventaire.

- Muséum d'Histoire naturelle de Bordeaux.

- Institut de Préhistoire et de Géologie du Quaternaire de l'Université Bordeaux I, UMR 5808 du CNRS. 


\section{Détermination du sexe}

9 Chez les diverses espèces du genre Bison connues à ce jour, dans l'ensemble les mâles sont plus grands, plus lourds surtout, que les femelles et leur tête, relativement plus large, s'orne de cornes plus développées. La seule observation des troupeaux d'individus vivant actuellement en Europe Centrale et en Amérique du Nord permet de noter de telles différences et les études statistiques conduites par Mc Donald (1981) en apporte la preuve non seulement en ce qui concerne le Bison américain actuel mais plusieurs formes fossiles. Il n'en reste pas moins vrai que la détermination du sexe à partir de pièces fossiles isolées et/ou endommagées se révèle fréquemment aléatoire. En revanche, lorsque certaines parties de la carcasse sont conservées, les chances d'aboutir à cette détermination semblent plus sérieuses, même en l'absence de tête osseuse.

10 En reconstituant les squelettes ou portions de squelettes découverts dans les secteurs $\mathrm{A}, \mathrm{B}$ et $\mathrm{E}$ de la grotte d'Habarra, on remarque que les rapports de dimensions entre certains os diffèrent sensiblement d'un sujet à l'autre, notamment la longueur du métacarpe en fonction de celle de l'humérus et du radius et la longueur du métatarse en fonction de celle du fémur et du tibia. Ceci nous a conduit à calculer la valeur de ces rapports chez des bisons actuels de sexe connu en utilisant les données de la littérature et les résultats de mensurations effectuées sur des spécimens conservés au British Museum, sur des squelettes conservés les uns au Laboratoire d'Anatomie Comparée du Muséum National, les autres à l'Institut de Préhistoire et de Géologie du Quaternaire de l'Université Bordeaux I.

11 Effectif utilisé :

12 Bison bonasus L. 1758 (Bison d'Europe) : 4 mâles et 4 femelles Bison bison L. 1758 (Bison d'Amérique) : 3 mâles et 4 femelles.

13 Chez les deux espèces considérées il apparaît que l'indice métacarpo-huméral et l'indice métacarpo-radial sont plus élevés chez les femelles et que l'indice métatarsofémoral et l'indice métatarso-tibial le sont souvent. Ci-après nous donnons les valeurs de ces indices calculées à partir de données des tableaux 1 et 2 , les deux espèces étant confondues :

\begin{tabular}{|l|l|l|}
\hline & Mâles & Femelles \\
\hline Indice métacarpo-huméral & $58,2-63,3$ & $64,5-74,1$ \\
\hline Indice métacarpo-radial & $58,7-62,5$ & $62,8-68,4$ \\
\hline Indice métatarso-fémoral & $58,3-65,5$ & $64,9-70,9$ \\
\hline Indice métatarso-tibial & $56,9-63,8$ & $60,2-68,3$ \\
\hline
\end{tabular}

14 Il est hautement probable que si nous avions disposé d'un nombre plus élevé d'individus de chaque groupe, les différences apparaîtraient moins nettes: des intervalles, ici bien distincts, pourraient alors se recouper tandis que d'autres se chevaucheraient davantage. 
15 Des diagrammes tracés selon la méthode de Simpson (1941 et Simpson et al, 1960) mettent bien en évidence les dissemblances entre mâles et femelles en ce qui concerne les rapports de longueur des grands os longs, en particulier la brièveté plus accusée des os canons chez les mâles (fig. 9 à 12). Sur ces diagrammes, on a utilisé comme valeur standard le logarithme de la longueur de l'os homologue chez le bœuf primitif (Bos primigenius) de la Prévalerie (Vallées des Eaux-Claires, Charente) étudié par Massaud (1984) auquel nous avons emprunté les termes de référence. Chaque point illustre la différence entre le logarithme de la longueur de l'os long considéré et le logarithme de l'os homologue standard. Le diagramme de la figure 13, construit de la même façon que les précédents, se rapporte aux squelettes ou portions de squelettes d'Habarra. Par comparaison avec les diagrammes des figures 9 à 12 , nous pouvons conclure sans plus attendre et sans risques excessifs que le bison $n^{\circ} 2$ des secteurs $A$ et $B$, particulièrement robuste d'ailleurs, était un mâle tandis que le bison $n^{\circ} 1$ des secteurs $A$ et les bisons $n^{\circ} 4$ et $n^{\circ} 5$ du secteur E étaient des femelles. Cela se trouvera corroboré par d'autres observations.

16 La lecture de ces diagrammes fait également apparaître des différences entre B. bison , B. bonasus et le Bison fossile d'Habarra mais elles seront traitées dans un chapitre ultérieur.

17 Un tableau élaboré par Mc Donald (1981, tabl. 30, p. 97) dans lequel figure la longueur moyenne des os longs chez divers bisons d'Amérique, actuels et fossiles, permet de calculer la valeur des quatre indices considérés plus haut. Les résultats, qui portent sur de très nombreux documents ( par ex. 164 métacarpes et 177 métatarses de femelles de l'espèce B. bison) confirment ceux obtenus à partir des mesures que nous avons prises, mais ils ne leur sont pas directement comparables parce que les humérus, radius, fémur et tibia n'ont pas été mesurés de la même manière.

\section{Détermination de l'âge}

Elle n'a été qu'approximative. Quand cela se révélait possible, nous avons pris en compte, outre le stade d'évolution de la dentition, le degré d'usure des prémolaires et molaires. A ce sujet nous soulignons qu'à ce jour très peu de dents isolées ont été recueillies à Habarra. Celles qui demeurent implantées dans les maxillaires et les mandibules, en général très usées, n'autorisent que des observations limitées.

19 Le degré d'usure des dents dépend de la nature des aliments. En effet, les végétaux riches en phytolithes (herbes de la prairie) usent plus vite la denture que les jeunes pousses d'arbres. Haynes (1984) a montré que l'abrasion des molaires est presque deux fois plus rapide chez les bisons américains des plaines que chez ceux du parc national de Wood Buffalo près d'Alberta et des territoires du Nord-Ouest qui vivent dans un environnement plus boisé. Chez ces derniers, la hauteur de la M1 inférieure diminue seulement de 1,5 mm par an. Le même auteur remarque aussi que la forme de la face occlusale des molaires diffère chez les deux populations. Chez les bisons des grands plaines elle tend, dans une certaine mesure, à s'aplanir alors que chez leurs congénères plus forestiers le sommet des cuspides reste, du moins plus longtemps, bien dégagé, le sillon vestibulo-lingual médian étant ainsi fortement encaissé (fig. 4, H1 et I3, H2 et I4). Se fondant sur des observations de Haynes, Guthrie (1990, p. 181) a calculé que chez le bison Blue Babe, découvert à l'état de momie près de Fairbanks en Alaska, dans des limons pléistocènes intercalés dans une couche tourbeuse, l'usure dentaire devait être 
particulièrement intense : 5,1 mm par an. Ce fossile, daté de $36425+2575 /-1974$ BP (QC-891), serait pratiquement identique au Bison des steppes eurasiatiques (loc. cit., p. 198). En l'état actuel des recherches nous ignorons dans quelle mesure l'environnement des bisons d'Habarra était compatible à celui de Blue Babe qui selon Guthrie (loc.cit. p. 183) se nourrissait essentiellement de plantes herbacées.

Une indication concernant l'âge d'un individu découle aussi parfois de la détermination du stade d'ossification du squelette : épiphyses soudées ou non à la diaphyse, ligne de suture des épiphyses, plus ou moins nette, continue ou discontinue. Nous considérons également la vigueur des reliefs d'insertion des muscles et ligaments qui s'affirme de plus en plus avec l'âge.

\section{Les bisons d'Habarra, BISON priscus Bojanus, 1827}

\subsection{Les secteurs A et $B$, gisement amont (fouilles, relevés et déterminations in situ André Clot pour le secteur A, J.-M. Barthe pour le secteur B)}

\subsubsection{Bison $\mathrm{n}^{\circ} 1 \mathrm{AB}$ femelle}

Dans le secteur A le bison était associé à Coelodonta antiquitatis; dans le secteur B, à ce même Rhinocéros et au Renne, Rangifer tarandus.

\subsubsection{Situation dans le gisement. Identification}

Le bison $n^{\circ} 1$ est essentiellement représenté par des os de son squelette appendiculaire qui, en majorité, gisaient dans le secteur $\mathrm{A}$, près de l'entrée supérieure, à l'intérieur d'une zone de forme à peu près carrée de $75 \mathrm{~cm}$ de côté. Ces pièces, ainsi que d'autres provenant du secteur $B$, ne se trouvaient pas en connexion anatomique et si quelques éléments de la patte antérieure gauche étaient bien groupés, dans l'ensemble les relevés de plan donnent l'image d'un certain désordre. A l'évidence tous ces restes se distinguent de ceux d'un autre bison, dit $\mathrm{n}^{\circ} 2$ nettement plus grand et plus puissant, découverts, eux aussi, partie dans le secteur A, partie dans le secteur B. Parmi les os manquants il faut citer les fémurs et le crâne dont on ne possède seulement, peut-être, qu'une portion de processus cornual (A 8) et des fragments non identifiables avec précision. Nous proposons d'attribuer au même bison $n^{\circ} 1$ deux mandibules du secteur B (B 5 et B 18) et une omoplate (B 6).

Si pour chaque membre les essais de mise en connexion anatomique des pièces sont limités en raison de l'absence de quelques éléments, ceux qui sont possibles se réalisent avec la précision souhaitable. Certes, le métacarpe gauche (A 38) ne peut être mis en rapport direct avec le radius et l'ulna gauches (A 37, A 6) parce que la plupart des carpiens de la même patte font défaut. Toutefois, parmi les ossements qui se trouvaient presque en contact de ces os longs, le pyramidal gauche $\mathrm{A} 40$ correspond bien au radioulnaire A 37-A 6. D'autre part les premières phalanges A 44 et A 45, présentes là aussi, s'ajustent parfaitement au métacarpe A 38. La comparaison des os homologues des pattes antérieures permet de conclure à une symétrie indiscutable, que l'on considère dimensions ou détails anatomiques, et il en est de même pour les os des pattes postérieures. Nous ne pouvons démontrer avec la même rigueur que les os des 
membres antérieurs et ceux des membres postérieurs se rapportent au même sujet puisqu'il n'existe pas de relations articulaires entre les uns et les autres. Nous retenons pourtant cette hypothèse pour les raisons suivantes :

- les rapports de longueur ( et autres dimensions) entre les os des membres antérieurs et ceux des membres postérieurs sont ceux que l'on s'attend à trouver chez un même individu ;

- chaque élément du squelette ne figure que par un spécimen unique ou deux spécimens symétriques ;

- ces différents os indiquent un animal robuste, de taille modeste comparativement à celle de la plupart des autres bisons d'Habarra, la vigueur des reliefs d'insertion dénotant un âge avancé;

- dans le lot d'ossements identifiés nous n'en voyons aucun donnant à penser qu'il existait dans les secteurs $\mathrm{A}$ et $\mathrm{B}$ les restes d'un autre bison à la fois moins grand que le bison $\mathrm{n}^{\circ} 2$, dont la présence a déjà été évoquée, et aussi vieux et massif que le bison $\mathrm{n}^{\circ} 1$. Les mandibules B 18 et B 5 (pl. 4) portent des dents très usées;

- toutes ces pièces associées en gisement possèdent incontestablement des analogies en ce qui concerne la densité et l'aspect.

24 Il est hautement probable que le bison $\mathrm{n}^{\circ} 1 \mathrm{AB}$ était une femelle comme tendent à le prouver les indices métacarpo-radial et métatarso-tibial respectivement égaux à 66,0 et 65,2 (tabl. 1, fig. 9 et 13).

L'identification du bison $\mathrm{n}^{\circ} 1$ se fonde sur le résultat de l'examen des os des membres. L'hypothèse selon laquelle des os crâniens et quelques vertèbres appartiendraient à cet individu semble très plausible mais nous ne possédons pas d'arguments irréfutables pour l'admettre sans réserve. Les dimensions de l'atlas (A19), bien conservé, figurent tableau 39.

Nous reviendrons ultérieurement sur le cas d'une mandibule gauche (B 88) aux dents relativement peu usées qui représente un sujet plus jeune et sans doute plus gracile que le bison $\mathrm{n}^{\circ} 1 \mathrm{AB}$ et sur celui d'un semi-lunaire gauche (A4) qui n'a pu, lui aussi, appartenir à cet individu.

\subsubsection{Eléments crâniens et vertèbres}

\subsection{Le processus cornual droit A 8}

Sa partie proximale et l'apex manquent mais la fracture distale paraît ne s'être produite qu'à une faible distance de ce dernier. Au total, la longueur de la pièce, dont la courbure est assez accentuée, atteint $192 \mathrm{~mm}$ en ligne droite. Quant à sa section, elle est franchement elliptique : à chaque extrémité le diamètre dorso-ventral l'emporte sur le diamètre rostro-caudal. Ils mesurent respectivement 59 et $50 \mathrm{~mm}, 43$ et $37 \mathrm{~mm}$. Des cannelures longitudinales bordées de crêtes plus ou moins accusées, visibles sur tout le pourtour mais plus larges et plus profondes sur la face ventrale et la face caudale, indiquent un sujet relativement âgé. Si au niveau de la fracture distale on n'observe pas de sinus, de telles cavités existent dans la région proximale dont la paroi compacte a une forte épaisseur : $18 \mathrm{~mm}$ du côté ventral.

\subsection{Les mandibules B 18 et B 5 (tabl. 7)}

Elles gisaient l'une près de l'autre dans le secteur $B$, donc un peu en contre-bas par rapport aux os des pattes du bison $\mathrm{n}^{\circ} 1$. On ne peut les replacer en connexion 
anatomique car elles sont détériorées dans leur partie rostrale mais leurs dimensions comparables, malgré quelques petits écarts, la morphologie et le degré d'usure des prémolaires et molaires homologues, identiques, prouvent qu'elles constituaient la mâchoire inférieure d'un même sujet: leur symétrie est parfaite dans ce qu'elles ont conservé en commun.

B 18 droite : une forte épaisseur de calcite recouvre sa branche montante et sa région angulaire. Le condyle et le processus coronoïde sont très endommagés.

B 5 gauche : mieux conservée que la précédente. Son processus coronoïde, relativement long et large, à peine entamé à son extrémité caudale, décrit une courbe accentuée audessus de l'incisure mandibulaire et du condyle qu'il dépasse très légèrement vers l'arrière. En revanche, la partie correspondant à l'angle de la mandibule a souffert. On remarque le relief prononcé de la zone d'insertion du masséter, en particulier la saillie de son bord rostral. Comme celui de la mandibule B 18 d'ailleurs, le corps est relativement haut et épais. Il convient de souligner la forte abrasion des dents jugales, toutes présentes. Elle avait atteint le fond des fossettes de M1 ce qui correspondrait chez les bisons d'Amérique actuels ou récents au stade d'usure 4 décrit et figuré par Skinner et Kaisen (1947, p. 146, pl. 13-4A “ old Age "). Si la face occlusale de la M1 est à peu près plane, celle des $\mathrm{M} 2$ et, surtout des $\mathrm{M} 3$, possède des cuspides en saillie, de part et d'autre du sillon vestibulo-lingual médian.

\subsection{Vertèbres déterminées}

$31 \quad 1$ atlas (A 19) bien conservé (tabl. 39), une 3ème( ?) cervicale fragmentaire (A 25-1), une 4ème cervicale (A 25-2), une 5ème cervicale (A 26) et une 6ème cervicale (A 27). Peutêtre faut-il rapporter au même bison une demi-thoracique T2 ou T3 ou T4 (A 17).

\subsubsection{La scapula gauche B 6 (tabl. 13)}

Elle a probablement appartenu au bison $n^{\circ} 1$ mais n'a pu être entièrement reconstruite à partir des quelques pièces (B 6, B 48, B 60) lui appartenant découvertes à moins de 15 $\mathrm{cm}$ des mandibules B 5 et B 18. La partie ventrale de l'os dont la face latérale disparaît sous une couche de calcite indique un animal de taille médiocre, comparativement à celle d'autres bisons figurant dans la caverne, mais ses dimensions semblent compatibles avec celles des éléments du bison $n^{\circ} 1$ (tabl. 13). Le bord de la cavité glénoïde s'infléchit en direction du centre de celle-ci, modérément mais de façon nette, tandis que le tubercule supra-glénoïdal apparaît relativement peu proéminent par rapport au bord crânial de la face articulaire, deux dispositions jugées comme caractéristiques du Bison (Stampfli, 1963). La partie dorsale est bordée d'un cartilage ossifié, ce qui dénote ( comme l'usure des dents des mandibules B5 et B18) un âge avancé.

\subsubsection{Les os des membres antérieurs}

\subsection{L'humérus droit A 32 (tableau 14)}

Il se trouvait sous l'atlas A 19, presque en contact avec son symétrique, l'humérus gauche incomplet A 10. 
distalement par rapport à sa gorge et par rapport au condyle (capitulum) ce qui, selon Stampfli (1963, fig. 9), est une des caractéristiques des humérus de Bison. En vue caudale, de part et d'autre de la fosse olécrânienne, l'épicondyle médial (épitrochlée) et surtout l'épicondyle latéral se révèlent plus arqués que sur les humérus de Bos auxquels nous les comparons. Cette remarque correspond à des observations de Stampfli (loc. cit), Massaud (1984) et Slott-Moller (1988) mais Brugal, dans le cadre de son étude sur les nombreux restes du Bœuf pléistocène de Lunel Viel (1983, p . 119), note que chez ce dernier la fosse olécrânienne est large avec des bords arrondis alors qu'elle est étroite avec des bords à peu près parallèles chez les bisons. Sur ce dernier point Brugal serait donc en désaccord avec les autres auteurs. En vue médiale, l'axe de l'os étant vertical, tandis que chez Bos la trochlée apparaît moins proéminente vers le bas que l'épicondyle médial, sur notre spécimen, ainsi que chez les bisons en général, l'une et l'autre se situent à peu près au même niveau. De ce fait le bord distal de l'humérus dessine, dans l'ensemble, une ligne presque droite sub-perpendiculaire à l'axe longitudinal de la diaphyse (fig. 6b). Ce critère de discrimination ainsi que le suivant ont été indiqués par Olsen (1960). En vue latérale, la dépression qui affecte le capitatum et qui loge l'extrémité proximale de l'extenseur du doigt latéral est relativement peu profonde et plutôt allongée dans le sens crânio-caudal. Le relief qui la limite vers le haut et l'arrière est peu proéminent. Chez le Bœuf cette fosse est profonde et a une forme circulaire. Le caractère observé chez le bison $n^{\circ} 1$ se retrouve en particulier sur les humérus des bisons $n^{\circ} 2$ et $n^{\circ} 4$ d'Habarra représentés figure $14-1$ et 2-. Comme dans la région proximale aucune ligne de suture des épiphyses n'est visible.

\subsection{L'humérus gauche A 10 (tableau 14)}

36 Au-dessus de la tubérosité deltoïdienne, sa région crâniale est détruite. Au-dessous il apparaît comme le symétrique parfait de l'humérus A 32.

\subsection{Radius et ulnas (tableaux 15 et 16)}

37 Le radius et l'ulna droits A 11 constituent un ensemble radio-ulnaire complet. Le radius et l'ulna gauche A 6 ont leur partie distale détruite. L'union des deux os est généralement moins intime chez le Bison que chez le Bœuf. Cela se manifeste surtout dans la région proximale, au-dessus et un peu au-dessous de l'espace interosseux, l'extrémité distale de celui-ci se situant au niveau du trou nourricier. En ce qui concerne les deux spécimens d'Habarra qui, rappelons le, représentent un sujet âgé, les deux os, indépendants au niveau de la face articulaire pour l'humérus, ne se soudent que $84 \mathrm{~mm}$ plus bas environ. La rainure, plus ou moins nette qui, assez souvent, sur la face articulaire sépare le radius de l'ulna chez les Bovinés, fait défaut sur le radioulnaire A 11.

\subsection{Les radius A 11 et A 37 (tableau 15, figure 15)}

38 Région proximale: En vue dorsale, le bord antérieur de la face articulaire est modérément mais franchement sinueux, comme cela se voit fréquemment chez les 
bisons (fig. 15-5), conformément à la remarque de Lehman (1949, fig. 8 et 9). Au sujet de la cavité glénoïde latérale, Delpech (1983, p.187) observe que chez Bos son bord palmaire et son bord dorsal (ou antérieur) sont presque parallèles tandis que chez Bison ils divergent du côté latéral. Sur les radius A 11 et A 37 d'Habarra c'est la disposition «Bison » qui prévaut mais le bord palmaire étant moins oblique que le bord dorsal la divergence des deux bords s'en trouve atténuée. Le relief latéral d'insertion, quelque peu anguleux vers l'extérieur, se révèle saillant tandis que sa face supérieure est déprimée, comme Bibikova (1958) et Stampfli (1963) l'ont constaté chez les bisons. Nous remarquons en outre que sur ces deux radius, comme sur les autres spécimens d'Habarra, la face proximale de ce relief, précisément celle sur laquelle se fixe le ligament collatéral médial du coude, regarde surtout vers le haut et du côté externe. Chez les bœufs elle s'oriente nettement du côté dorsal (fig. 15-5).

Région distale : Sur la face dorsale, la dépression longitudinale pour l'extenseur radial du carpe est peu encaissée; les deux reliefs qui la limitent ne sont guère plus proéminents l'un que l'autre. Sur la face palmaire, comme il est de règle chez les bisons, le bord médial du condyle interne est relativement peu oblique de bas en haut dans le sens médio-latéral : les deux bords de cette face articulaire qui répond au scaphoïde sont sub-parallèles alors qu'ils convergent vers l'arrière chez les bœufs (Olsen 1960).

\subsection{Les ulnas A 11 et A 6}

En vue latérale et médiale, l'axe principal de l'olécrâne décrit avec celui du radius un angle moins ouvert que chez Bos. Caractère observable sur l'ulna gauche dissocié du radius A 37 mais bien conservé dans sa région proximale : les deux facettes dorsales en contact avec les facettes correspondantes de l'échancrure palmaire du radius réalisent un angle obtus (aigu chez le Bœuf d'après Stampfli, loc. cit.) alors que la facette en relation avec l'humérus forme un angle droit. En vue latérale ou médiale, le bord dorsal de l'olécrâne (A 11 et A 6) (tabl. 16) décrit une large convexité et n'ébauche au niveau du bec qu'une faible convexité, ce qui constitue un caractère "Bison " selon Stampfli.

\subsection{Le pyramidal gauche A 40 (tableau 17)}

41 En vue latérale (ou dorsale) le bord proximal du condyle en relation avec le radius paraît arrondi et non anguleux comme il devrait l'être, en principe, chez un bison ( Stampfli, loc. cit., fig. 19) mais il faut dire que le carpien A 40 est quelque peu endommagé dans la région considérée. D’autre part, le bord latéral de la face articulaire qui rencontre l'os crochu décrit une concavité modérément prononcée, juste en avant de la saillie disto-palmaire.

\subsection{Le semi-lunaire gauche A 104 (tableau 18, figure 16-3)}

En vue dorsale, la face articulaire proximale apparaît assez fortement bombée et légèrement asymétrique et le bord latéral de la face antérieure est concave. D'après Stampfli (1963, p.137, fig. 17) cette disposition ne serait pas précisément caractéristique du Bison. Le rapport centésimal hauteur-largeur a souvent été calculé pour les semi-lunaires mais une certaine confusion a longtemps régné en ce qui concerne l'interprétation des résultats car les comparaisons entre les genres Bos et Bison ne portaient pas toujours sur des indices évalués de la même façon : termes du rapport inversés, mesures non concordantes... Sans revenir longuement sur la 
question, nous suivrons Slott-Moller (1988, p. 46 ; tabl. 19 et 20) qui a bien clarifié la question. Si l'on considère la hauteur dorsale et le diamètre transversal dorsal proximal, on constate qu'en général les semi-lunaires des bisons sont plus larges que hauts. C'est le cas du semi-lunaire A 104 et, à une exception près, des autres spécimens d'Habarra. Quant aux semi-lunaires des bœufs ils sont, en moyenne, plus hauts que larges. Un autre semi-lunaire gauche, A4 (tabl. 18), se trouvait parmi les ossements du bison $n^{\circ} 1$ mais il doit être rapporté à un individu plus petit.

\subsection{Le métacarpe gauche A 38 (tableau 22, figures 17-4 et 17-9)}




\subsection{Deuxième phalange antérieure A 43 (tableau 24, figure 19-4, 5 et 6)}

48 connexion anatomique au moment de leur découverte. Elle présente les caractères propres aux deuxièmes phalanges antérieures des Bovinés. Elle est courte, massive et la dissymétrie de l'os se révèle accusée, notamment en vue distale, le condyle abaxial étant plus développé par rapport à l'axial que sur les deuxièmes phalanges postérieures.

\subsubsection{L'os coxal gauche A 1 (tableau 25)}

La seule partie mesurable se situe au niveau du col de l'ilium mais on remarque que les os coxaux ont été séparés avant leur découverte par cassure et non par désarticulation et que l'épiphyse de la tubérosité ischiatique était parfaitement soudée

\subsubsection{Les os des membres postérieurs}

\subsection{Les tibias A 15 droit et A 16 gauche (tableau 27, fig. 20)}

Ils gisaient l'un sur l'autre. A 15, dont l'extrémité proximale était sous-jacente au radioulnaire A 11, recouvrait partiellement la partie distale de l'humérus gauche A 10. Tous deux ont subi certains dommages surtout dans la région latérale du plateau tibial, ce qui n'empêche pas de constater l'absence de tout rudiment de fibula à extrémité libre aiguë au-dessous du condyle externe. On sait qu'un petit vestige de péroné adhérant à ce condyle, la pointe dirigée vers le bas, existe très souvent chez les bœufs mais pas chez les bisons. En tout cas nous ne l'avons jamais observé chez Bison priscus.

51

Notre attention s'est portée sur les crêtes d'insertion du poplité et des fléchisseurs des doigts qui ornent la face caudale. Olsen (1960, p. 12), qui les a numérotées de 1 à 4 dans le sens latéro-médial, note que chez Bos les trois premières convergent vers un même point ( sans vraiment l'atteindre) situé assez près du bord proximal du plateau tibial. Massaud (1984, p. 43) constate à peu près la même chose chez l'aurochs de la Prévalerie mais signale une petite différence entre le tibia droit et le tibia gauche, ce dernier se singularisant par une crête $n^{\circ} 3$ moins allongée vers le haut. Selon ces auteurs, chez le Bison, la crête $\mathrm{n}^{\circ} 3$ est nettement plus courte que les crêtes $\mathrm{n}^{\circ} 2$ et $\mathrm{n}^{\circ} 1$, son extrémité proximale restant plus éloignée du plateau tibial. Sur la face caudale des spécimens A 15 (fig. 20-1) et A 16 d'Habarra, nous observons quatre crêtes vigoureuses relativement allongées ( surtout les $n^{\circ} 1$ et 3 ) et bien nettes. L'extrémité proximale des crêtes $n^{\circ} 2$, $\mathrm{n}^{\circ} 3$ et $\mathrm{n}^{\circ} 1$ aboutissent relativement près du plateau tibial (aussi près que chez Bos) mais celle de la crête $n^{\circ} 4$ en est plus éloignée. Aucun de ces quatre reliefs principaux ne fait fortement saillie dans la moitié distale de l'os. Cependant, la plus longue, la crête $\mathrm{n}^{\circ} 1$, se prolonge vers le bas, timidement, puis disparaît presque totalement dans la région correspondant à l'épiphyse distale. La crête $\mathrm{n}^{\circ} 2$, courte mais proéminente dans sa partie proximale, s'efface vers le milieu de l'os, réapparaît un peu plus bas sur une courte distance en restant parallèle à la crête $n^{\circ} 1$. Lorsqu'on compare les tibias A 15 et A 16 aux trois tibias de Bison actuel d'Amérique conservés à l'Institut de Préhistoire et de Géologie du Quaternaire de l'Université Bordeaux I (fig. 21-2 à 4) on constate que sur les deux os fossiles, dans la région d'insertion du poplité, les crêtes $n^{\circ} 4$ et $n^{\circ} 3$ sont plus courtes et beaucoup plus obliques de haut en bas dans le sens latéro-médial. L'extrémité distale de la crête $\mathrm{n}^{\circ} 4$ rencontre le bord médial de la diaphyse nettement

PALEO, 15 | 2003 
plus haut que le milieu de l'os et celle de la crête $n^{\circ} 3$ un peu au-dessous de ce niveau. Sur les tibias de Bison d'Amérique précédemment cités, la crête $\mathrm{n}^{\circ} 4$ rejoint le bord médial plus bas que le milieu du tibia tandis que la crête $n^{\circ} 3$ l'atteint plus bas encore ou même ne l'atteint pas (fig. 21-2 à 4). Néanmoins il n'en est pas toujours ainsi puisque sur les tibias d'un squelette de Bison bison exposé dans la galerie du Muséum d'Histoire Naturelle de Paris, les crêtes $n^{\circ} 4$ et $n^{\circ} 3$ sont aussi courtes et aussi obliques que chez le bison $n^{\circ} 1$ d'Habarra. En revanche, les tibias d'une petite femelle de Bison américain dont le squelette appartient aux collections du Laboratoire d'Anatomie comparée du Muséum National présentent une crête $n^{\circ} 4$ relativement longue et peu oblique qui forme avec la crête $n^{\circ} 3$ une longue dépression longitudinale assez semblable à celle que l'on voit sur les trois spécimens de Bison américain représentés figure 21. L'ensemble des examens que nous avons pratiqués ne nous permet certes pas d'affirmer qu'il existe une différence entre B. priscus et B. bison en ce qui concerne la zone d'implantation du poplité mais il invite à poursuivre les observations à ce sujet. Chez le Bœuf (fig. 21-1) les crêtes $n^{\circ} 4$ et $n^{\circ} 3$ sont au moins aussi courtes et aussi

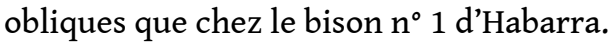

A l'extrémité du tibia A 15 les deux facettes pour l'os malléolaire, non décalées en hauteur l'une par rapport à l'autre, sont profondément séparées l'une de l'autre (caractères "Bison " d'après Bibikova et Lehmann) et non reliées par un " pont " (caractère "Bos"). Cependant d'après Brugal (1983) il arrive que chez le Bœuf les dites facettes " communiquent ". Cet auteur, à l'appui de sa remarque, donne des exemples précis (p. 267, fig. 50) et signale aussi que chez le Bison la facette antérieure est " ronde et bombée tandis que chez le Bœuf elle est plate et s'incline sur le côté".

\subsection{Les os du tarse}

De nombreux auteurs, Lehmann (1949), Bibikova (1958), Olsen (1960), Stampfli (1963) ont comparé le tarse des bisons à celui des bœufs et mis en évidence un certain nombre de différences entre les deux genres. Nous n'en rappellerons qu'un petit nombre à propos de 3 tarsiens attribuables au bison $n^{\circ} 1$ d'Habarra.

\subsection{Talus droit A 23 et talus gauche A 24 (tableau 32)}

Le bord proximo-médial de la dépression située entre la trochlée plantaire et la trochlée distale est arrondi en arc de cercle ( caractère « Bison »).

\subsection{Calcanéum droit A 12 et calcanéum gauche A 39 (tableau 33)}

Juste au-dessus de la facette articulaire répondant au cubo-naviculaire, la face plantaire est régulièrement arrondie vers le bas et l'arrière. Chez le Bœuf, au même endroit, elle présente un méplat orienté latéralement.

\subsection{Cubo-naviculaire gauche A 18 (tableau 30)}

Découvert sous le tibia gauche A 16, à $12 \mathrm{~cm}$ du talus A 24 avec lequel il s'articule bien, il est endommagé au-dessous de l'angle dorso-médial de la face articulaire proximale. 


\subsection{Métatarse droit A 34 et métatarse gauche A 13 (tableau 34)}

57 Le métatarse A 34 était en partie recouvert par l'atlas A 19 et l'humérus A 32 euxmêmes sous-jacents aux tibias A 15 et A 16. Le métatarse droit est sérieusement détérioré à son extrémité distale: sa longueur totale ne peut être mesurée. Son symétrique a seulement subi quelques petits dégâts dans la même région, du côté latéral. La plupart des mensurations ont été effectuées mais nous ne pouvons juger du développement des tubercules distaux sus-articulaires par rapport à la largeur de la face articulaire. Cependant le calcul de l'indice de robustesse traduit, comme dans le cas de l'os canon antérieur, le caractère massif de la patte. En vue dorsale, on remarque la forte saillie vers l'avant de la partie qui correspond au métatarsien III. Cette proéminence longe le sillon médian qui se trouve ainsi accentué.

\subsection{Premières phalanges postérieures A 35, A 84 et A 21 (tableau 35, figure 18)}

Toutes trois ont des dimensions comparables. A 35 (fig. 18-8 et 9) et A 84 s'ajustent parfaitement au métatarse A 13.

\subsection{Deuxième phalange postérieure A 30}

Ses dimensions apparaissent sur le tableau 36 et sa morphologie sur la figure 19-10.

\subsubsection{Le sujet $n^{\circ} 1 \mathrm{AB}$, conclusions}

60 Sa caractérisation reste incomplète. De nombreux éléments de son squelette font défaut et, parmi eux, le crâne et vertèbres qui jouent un rôle important dans la définition des espèces. Ce qui est indubitable c'est son appartenance au genre Bison (et à l'espèce priscus) puisque ses os en présentent les caractéristiques. Il n'est pas moins sûr qu'il s'agit d'une femelle dont l'âge avancé est attesté par le degré d'usure des molaires, les fortes traces d'insertion des muscles et ligaments et la minéralisation du cartilage scapulaire de l'omoplate $\mathrm{B} 6$, dans l'hypothèse où cet os se rapporte au même sujet que les autres vestiges que nous venons d'évoquer. Si l'on s'en tient aux os pour lesquels la littérature offre le plus de renseignements précis, les métacarpes et métatarses par exemple, on est tenté de dire, en se référant aux chiffres fournis par McDonald (1981, tabl. 30, p. 97), que la femelle $1 \mathrm{AB}$ d'Habarra était aussi grande que les plus grandes femelles du Bison des plaines actuel d'Amérique du Nord. Nous constaterons ultérieurement que, en comparaison de celles des autres bisonnes d'Habarra, sa stature se révèle seulement médiocre. Un des traits les plus remarquables $\mathrm{du}$ squelette $\mathrm{n}^{\circ} 1$ réside dans le caractère massif de ses divers composants, plus spécialement celui des os canons, plus accusé que chez les bisons femelles d'Amérique du Nord et d'Europe (tabl. 1).

\subsubsection{Bison $n^{\circ} 2 \mathrm{AB}$ mâle}

\subsubsection{Situation dans le gisement. Identification}

61 Les pièces du squelette du bison $n^{\circ} 2$, parmi lesquelles le crâne (A55-B1) et une mandibule (A 83), ont été recueillies en nombre assez important, disséminées dans les secteurs $A$ et $B$, mêlées par endroits aux ossements du bison $n^{\circ} 1^{3}$. Cependant, la lecture des plans montre que leur dispersion s'est réalisée dans un certain ordre. En effet elles 
se sont disposées suivant la pente de l'éboulis, dans le sens nord-sud, de telle sorte que les os des membres postérieurs se trouvaient dans le secteur A et ceux des membres antérieurs, ainsi que le crâne, soit dans ce même secteur mais en des points de la déclivité situés plus bas, soit dans le secteur B.

Nous rappelons que la distinction des deux individus bien représentés dans la zone A-B s'est révélée facile tant les ossements du bison $n^{\circ} 2$ diffèrent de ceux du bison $n^{\circ} 1$ par leurs dimensions beaucoup plus fortes. Cet animal à coup sûr était un mâle dont les os canons, surtout les antérieurs, sont particulièrement trapus. L'indice de robustesse du métacarpe droit atteint 25,1 et celui du métacarpe gauche 25,3 ce qui correspond à des valeurs élevées chez les bisons mâles y compris ceux de l'espèce priscus ( tabl. 2 et 22 ; fig. 17-1). L'indice métacarpo-radial égal à 60,5 et l'indice métatarso-tibial égal à 60,9 sont faibles (tabl. 2), plus faibles qu'ils ne le sont chez les femelles. D'autre part, les dimensions de la tête osseuse (A 55-B 1) s'harmonisent nettement mieux avec celles des os des membres du bison $n^{\circ} 2$ qu'avec celles des os du bison femelle $n^{\circ} 1$. Utilisant les chiffres publiés par McDonald (1981, tabl. 29 et 30) pour l'espèce B. bison, nous avons évalué le rapport centésimal moyen de la longueur du métatarse (par exemple) à la longueur du crâne. Il diffère assez peu en moyenne de 47,6 chez les mâles et de 50,5 chez les femelles. Pour les os correspondants des quelques bisons d'Europe ayant fait l'objet du même calcul et dont les dimensions figurent dans nos tableaux 1, 2, 7 et 8 nous avons obtenu des valeurs comprises entre 47,8 et 54,5 chez les mâles et entre 51,7 et 53,9 chez les femelles. En ce qui concerne le bison $n^{\circ} 2 \mathrm{AB}$, si l'on admet que le crâne A55-B1 lui appartient, l'indice égale 45,6, valeur peu éloignée de la valeur moyenne chez B. bison. En revanche, si nous associons le crâne aux os des membres du bison $n^{\circ} 1$ $A B$, nous obtenons une valeur certainement trop faible : 41,6.

6.1.2.2 Le crâne A55-B1 (tableaux 3, 4, 5 et 6) (pl. 1, 2 et 3) 
Planche 1 - Bison priscus Habarra. Crânes en vue frontale de cinq individus : 3 mâles (2AB, 12Jd, $14 \mathrm{Jc}$ ) et 2 femelles (4E, 6F).

Plate 1 - Bison priscus Habarra. Frontal view of the skulls of five individuals: 3 males (2AB, 12Jd, 12Jc) and 2 females (4E, 6F).

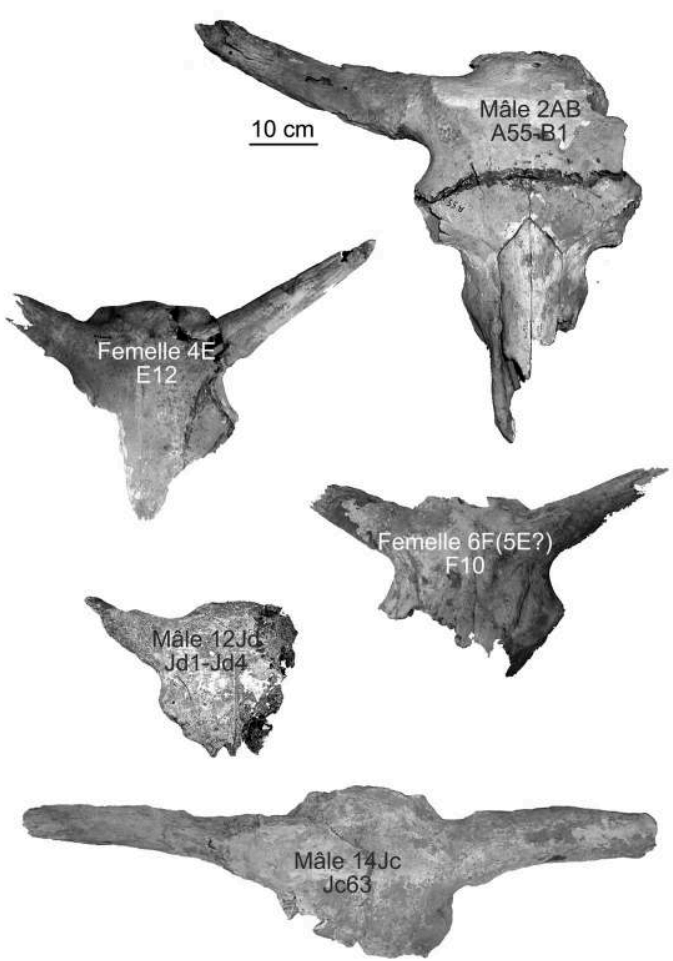

A leur extrémité distale, les processus cornuaux ne montrent pas d'ébauche de torsion vers l'arrière comme fréquemment chez B. bonasus et B. bison. Noter aussi que les processus cornuaux réalisent au départ, un angle caudal relativement au plan sagittal plus ouvert chez le mâle que chez la femelle. The distal ends of the horn cores show no trace of the twisting toward the rear that is common in $B$. bonasus and B. bison. Also note that, in relationship to the sagittal plane, the base of the horn cores of the males is marked by a broader caudal angle than is that of the females. 
Planche 2 - Bison priscus Habarra. Crânes en vue nucale de cinq individus : 3 mâles (2AB, 12Jd, $14 \mathrm{Jc}$ ) et 2 femelles (4E, 6F).

Plate 2 - Bison priscus Habarra. Nuchal vie of the skulls of five individuals: 3 males $(2 \mathrm{AB}, 12 \mathrm{Jd}, 12 \mathrm{Jc})$ and 2 females (4E, 6F).

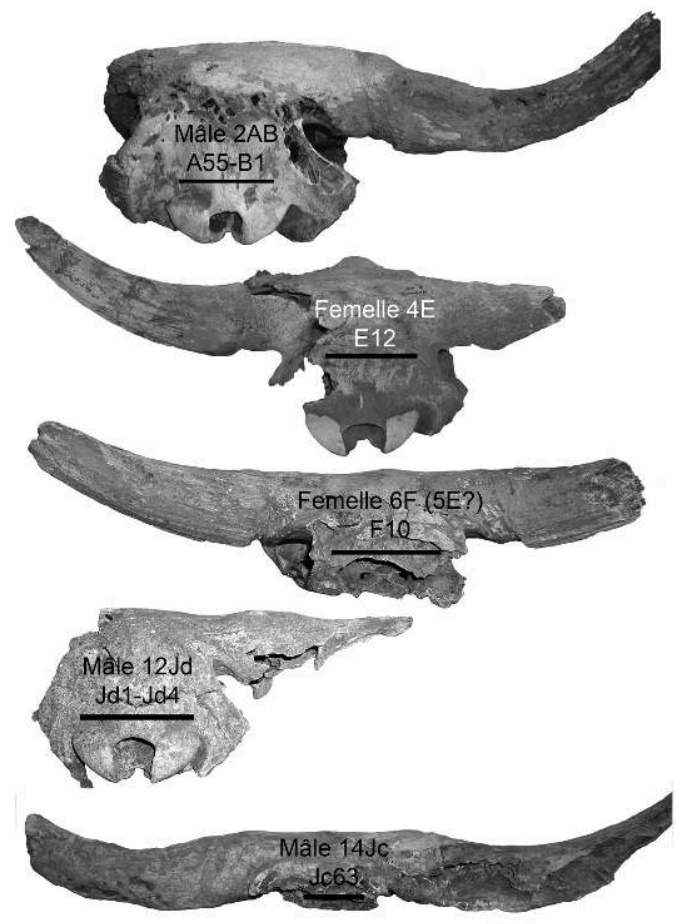

Echelle différente selon l'individu : le trait noir tracé sur chacun des crânes représente $10 \mathrm{~cm}$. Les chevilles osseuses de cornes, moins développées chez la femelle, sont aussi plus nettement dressées dès leur base.

The scales differ from individual to individual; the black line on each represents $10 \mathrm{~cm}$. Compared to those of the male, female horn cores are less well developed and emerge more horizontally from their base. 
Planche 3 - Bison priscus Habarra. Crânes en vue de profil de trois individus : 1 mâle (2AB) et 2 femelles (4E, 6F).

Plate 3 - Bison priscus Habarra: Profile views of the skulls of three individuals: 1 male (2AB) and 2 females $(4 E, 6 F)$.

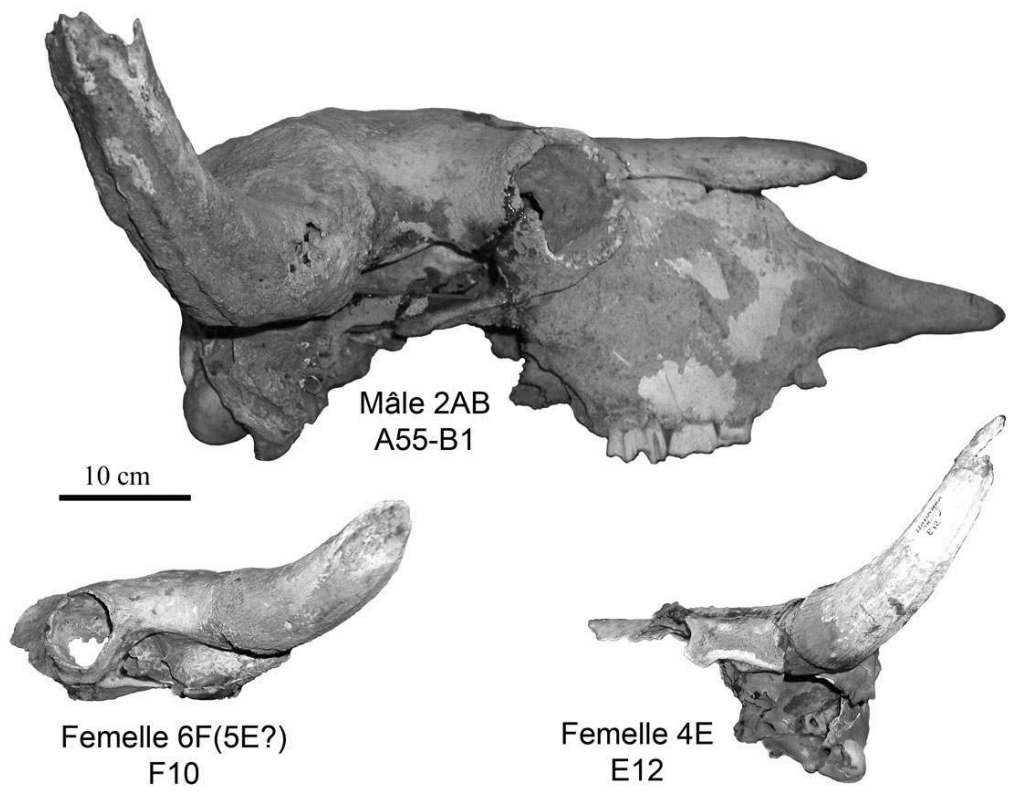

Chez le mâle, les processus cornuaux dans leur partie proximale, tout en s'écartant du plan sagittal, se dirigent vers l'arrière et le bas comme ployant sous leur propre poids. Les points les plus ventraux de la grande concavité médio-dorsale dépassent vers le bas le plan des frontaux; ce n'est pas le cas chez la femelle.

In leaving the sagittal plane, the proximal aspect of the male hore core is directed down and toward the rear, as if bending from its own weight. The ventral-most points of the large medio-dorsal concavity fall beneath the frontal plane in the male, but not in the female.

Il n'a pas été recueilli entier mais, pour l'essentiel, en trois parties. Deux d'entre elles, qui se trouvaient dans le secteur A (A55), ont été dégagées par André Clot. Chacune correspondait à une " moitié " du massif facial aujourd'hui reconstitué après réajustement des deux pièces suivant le plan sagittal. L'une, la gauche, comprenait notamment l'orbite (complète) mais pas l'os incisif et l'autre l'os incisif et les deux tiers de l'orbite. En fait l'os incisif gauche, fortement concrétionné, adhérait à la paroi interne du maxillaire supérieur droit. La partie crânienne proprement dite (B 1) gisait dans le secteur B où J.-M. Barthe la mit au jour. Elle a rejoint le bloc facial reconstruit en arrière du bord supra-orbitaire gauche, suivant un plan à peu près perpendiculaire au plan sagittal. La jonction des deux ensembles a permis de compléter le pourtour de l'orbite droite. Les deux os nasaux, à peine enterrés non loin des pièces précédentes, ont retrouvé leur place.

Du côté ventral les dégâts concernent la région comprise entre les palatins, dont les lames perpendiculaires n'existent plus, et la partie basilaire de l'occipital. Du côté gauche le maxillaire supérieur est détruit en avant de la quatrième prémolaire. Cette dent, ainsi que M1, M2 et M3 sont conservées mais restent informes sous un épais revêtement de calcite. Du côté droit, la couronne des M1, M2 et M3, seules dents présentes, a été dégagée. L'emplacement du bord mésial de P2 est déterminable. 

la partie basale de son occipital endommagé et la face ventrale des condyles.
Dimensions (à l'exclusion des processus cornuaux). Proportions.

66 cornuaux (tabl. 3-1 et 11) on constate qu'elles dépassent respectivement celles relevées par McDonald (1981, tabl. 29 et 34) sur des crânes de l'espèce B. bison, les deux sexes confondus. De même, aucun des crânes de Bison d'Europe, B. bonasus, que nous avons mesurés ou dont nous rapportons les dimensions d'après Degerbol et Iversen (1945, tabl. 1), n'est aussi grand (tabl. 7 et 8). Cependant, des crânes de B. priscus, plus grands que le crâne d'Habarra, ont été signalés en Europe. Nous citerons le spécimen de la grotte des Bisons à Lurbe Saint-Christau dans les Pyrénées Atlantiques (tabl. 3, 4 et 5), celui de Riverenert (Ariège) examiné par Crouzel et al. (1982) et ceux (dont certains mesurés par La Baume), à sexe non indiqué, publiés par Degerbol et Iversen (1945, tabl. 1) (tabl. 6).

67 D'autre part, dans ses proportions, le crâne A55-B1 se rapproche plus, semble-t-il, du crâne des bisons mâles de l'espèce européenne actuelle que de celui, relativement plus large, des bisons d'Amérique actuels. C'est en tout cas ce qui ressort de la comparaison de l'indice de la largeur frontale entre orbites et processus cornuaux en fonction 1) de la longueur saillie nuchale-bords antérieurs des os incisifs, 2) de la longueur saillie nuchale-épine frontale (tabl. 3, 8 et 10).

\section{Les os frontaux-processus cornuaux}

68 l'ensemble conformément à ce que l'on observe souvent chez Bison priscus d'après McDonald (loc. cit., p. 114). Ceux de la plupart des crânes des bisons actuels apparaissent fréquemment plus ou moins bombés. En fait, un examen du profil supérieur du calvarium A55-B1 (pl. 3) montre, de l'arrière vers l'avant, à partir du col du processus cornual, une légère convexité qui ne dépasse pas le milieu de l'orbite puis une faible ensellure fronto-nasale. Cette dernière précède une convexité nasale modérée mais nette. En vue frontale (pl. 1), on décèle un très léger bombement transversal entre les deux sillons supra-orbitaires. Entre chacun de ces sillons et le bord supérieur de l'orbite adjacente l'os frontal est tout à fait plat. qu'une faible partie de son extrémité distale mesurée suivant la courbure supérieure, $\mathrm{du}$ bord dorsal de la couronne à l'apex, égale $430 \mathrm{~mm}$. C'est plus que la longueur moyenne correspondante des chevilles osseuses des sujets mâles $(n=36)$ de l'espèce priscus calculée par McDonald (loc.cit., tabl. 38) : 362,7 $\pm 5,9$ (tabl. 6). C'est moins que la longueur des chevilles osseuses du crâne découvert dans la grotte des Bisons à LurbeSaint-Christau daté de $20730 \pm 710$ ans BP (Ly 2452) ${ }^{4}$. Elle atteint en effet $542 \mathrm{~mm}$ sur ce spécimen (tabl. 5).

70 Le processus cornual gauche est fortement endommagé. Néanmoins, si l'on suppose que les deux chevilles osseuses étaient à très peu près symétriques, ce qui en l'occurrence semble tout à fait plausible, la distance entre la pointe reconstituée du processus droit et le plan médian matérialisé ne diffère guère de $525 \mathrm{~mm}$; on en déduit que $1050 \mathrm{~mm}$ environ séparaient les deux apex. Nous sommes loin des $2000 \mathrm{~mm}$ correspondant à l'évaluation de cette dimension par Crouzel et al (1982) pour le crâne A incomplet 
recueilli dans la caverne de Riverenert, ou même des 1273 mm du crâne de Lurbe-SaintChristau (tabl. 5).

71 Chez le bison $n^{\circ} 2$ d'Habarra, le processus cornual droit, dans son mouvement de latéralité, se dirige d'abord vers le bas et quelque peu vers l'arrière en réalisant un angle caudal d'environ $72^{\circ}$ avec le plan sagittal : ses points les plus caudaux se situent légèrement au-delà du plan de l'occipital. Il s'élève ensuite fortement en décrivant une courbe accusée à convexité médiale, les points les plus ventraux de celle-ci demeurant pourtant franchement au-dessous du plan des os frontaux (pl. 2). Finalement, l'apex reconstitué se situe relativement haut par rapport à ceux-ci, comme cela se voit sur le spécimen A de la grotte de Riverenert représenté par Crouzel et al (loc.cit., fig. 5). Les chevilles osseuses du crâne de Lurbe-Saint-Christau, plus déployées, ont un apex moins élevé.

$\mathrm{Vu}$ perpendiculairement à sa face latérale (pl. 3), dans son mouvement ascendant, le processus cornual ne s'incline sensiblement ni vers l'avant ni vers l'arrière. Rappelons que le crâne repose sur ses mâchelières et la base de ses condyles occipitaux. Crouzet et al. (loc.cit., p. 78) signalent que le processus cornual du crâne de Riverenert se redresse verticalement en restant dans le même plan.

Le processus cornual droit du crâne A55-B1, dont la longueur a fait l'objet d'une évaluation jugée satisfaisante, a pourtant subi divers dommages. A la destruction de son extrémité apicale s'ajoutent des dégâts affectant sa face dorsale ainsi que ses faces ventrale et caudale. D'autre part, une croûte de calcite, non négligeable par endroits, recouvre sa région proximale. On ne peut donc suivre l'évolution du rapport des diamètres rostro-caudal et dorso-ventral de façon très précise. Il semble cependant que le premier soit à peu près égal ou à peine inférieur au second au niveau de la couronne (respectivement $106 \mathrm{~mm}$ et $107 \mathrm{~mm}$ ) et à $10 \mathrm{~cm}$ de celle-ci $(85 \mathrm{~mm}$ et $92 \mathrm{~mm}$ ). A $23 \mathrm{~cm}$ environ le rapport s'inverse: c'est le diamètre rostro-caudal qui l'emporte: $63 \mathrm{~mm}$ contre $55 \mathrm{~mm}$. Plus haut la différence s'accentue encore dans le même sens $(60 \mathrm{~mm}$ $44,5 \mathrm{~mm}$ ). En même temps le diamètre rostro-caudal maximum, d'abord parallèle au plan sagittal, devient légèrement oblique par rapport à celui-ci, de l'avant vers l'arrière et dans le sens latéro-médial. Cette disposition devait tendre à orienter la région apicale légèrement vers l'avant. Force est d'admettre, nous le répétons, que cela n'apparaît guère en l'état de conservation de la cheville osseuse.

L'appendice cornual étant creux à hauteur de la cassure, il est vraisemblable que le diverticule du sinus frontal devait aboutir à proximité de l'apex.

Quant aux cannelures longitudinales bordées de reliefs plus ou moins vigoureux qui, à partir d'un certain âge, ornent les processus cornuaux, nous en observons plusieurs. L'une d'elles, délimitée par deux crêtes aiguës, parcourt la face ventrale en position médiale. Elle est flanquée de deux cannelures adjacentes relativement larges : l'une rostro-ventrale, l'autre caudo-ventrale. Il existe également deux cannelures moins accusées sur la face caudale.

Les orbites

76 Chacune est située relativement plus près de la base de la cheville osseuse correspondante que chez le Bœuf mais faute de mesures précises nous ignorons si elle en est plus ou moins éloignée que chez Bison bison ou chez Bison bonasus. Son rebord paraît fortement proéminent, surtout dans ses parties dorso-caudale et latérale, même si l'on tient compte de la croûte de calcite qui accentue ce caractère. Les concrétions et 
quelques dommages subis par l'arc caudal de l'orbite droite et l'arc dorsal de l'orbite gauche nous contraignent à ne considérer les dimensions indiquées tableau 3 (8,15 et 16) qu'avec une certaine réserve. On constate également que l'arc de l'orbite réalisé par l'os frontal est plus étroit que celui formé par l'os lacrymal et l'os zygomatique. D'autre part, l'arc dessiné par l'os jugal est plus étroit que celui déterminé par le lacrymal et le frontal.

Les os lacrymaux

77 Leur extrémité rostrale, qui se trouve à l'aplomb de M1, présente une nette ensellure dorso-latérale. Ils se révèlent allongés et leur plus grande largeur semble se situer dans la région orbitaire. On serait tenté de dire qu'ils sont relativement étroits mais on suit mal le bord ventro-caudal en partie dissimulé sous un dépôt de calcite.

\section{Les os nasaux}

sont longs, modérément larges, bombés transversalement; le contour de chacun s'inscrit assez bien dans un rectangle. Le droit, intact, long de $235 \mathrm{~mm}$, est presque aussi large au point où son bord latéral perd tout contact avec le maxillaire supérieur $(92 \mathrm{~mm})$ qu'au point où il touche à la fois l'os lacrymal et l'os frontal $(98 \mathrm{~mm})$. D'après McDonald (1981, p. 114 et 115) les os nasaux des mâles de l'espèce priscus sont larges et rectangulaires tandis que ceux des femelles sont étroits et triangulaires.

L'os nasal droit, bien conservé, possède une extrémité rostrale bilobée et, conformément à ce que l'on constate chez les bisons, le lobe médial, aigu, dépasse vers l'avant le lobe latéral pourtant bien formé ici. On sait que chez le genre Bos la pointe latérale dépasse vers l'avant (parfois de peu) la pointe médiale. L'os nasal gauche est amputé de sa région apicale sur une longueur de $55 \mathrm{~mm}$ environ.

La crête faciale de l'os zygomatique, saillante et aiguë légèrement en arrière de l'orbite, tend peu à peu, vers l'avant, à s'effacer au-dessous de la partie la plus ventrale de cette cavité pour réapparaître timidement à l'aplomb de son bord rostral. Devenant plus nette elle rejoint vers le bas le tubercule facial, en décrivant une convexité dorsorostrale.

81 Lieu d'insertion le plus rostral du masséter, le tubercule facial du maxillaire supérieur, à la fois allongé et épais, proéminent vers l'arrière, se situe en son milieu à $6 \mathrm{~cm}$ environ au-dessus du bord alvéolaire latéral de l'espace interdentaire M1-M2, relativement plus haut et un peu plus en arrière que sur les crânes de Bœuf utilisés pour comparaisons. Il se prolonge vers le bas et l'avant par une crête saillante avec laquelle il dessine une convexité ventro-caudale. Cette crête se termine à $1,5 \mathrm{~cm}$ audessous du foramen infra-orbitaire, à l'aplomb de P2. En avant de la région d'implantation de cette dent le maxillaire supérieur est fortement déprimé.

Les os incisifs

82 L'os incisif gauche est détruit mais le droit est seulement un peu endommagé à son extrémité rostrale. Comme il est de règle chez le Bison et contrairement à ce que l'on voit chez le Bœuf, son extrémité caudale n'atteint pas l'os nasal. Elle parvient seulement, approximativement, à l'aplomb du milieu du foramen infra-orbitaire et de l'espace inter-dentaire P2-P3, à $35 \mathrm{~mm}$ du point le plus rostral de la ligne de contact du maxillaire supérieur avec l'os nasal, à $63,5 \mathrm{~mm}$ du point de contact nasal-lacrymalmaxillaire supérieur et à $228 \mathrm{~mm}$ du point où convergent nasal, lacrymal et frontal. Quant à la distance entre l'extrémité rostrale de chaque os incisif et le point où, vers l'avant, os nasal et maxillaire supérieur se séparent, elle mesure $212 \mathrm{~mm}$. 

de l'os nasal et le bord rostral de l'os incisif : $152 \mathrm{~mm}$. L'os incisif étant détruit, nous avons évalué la largeur de l'ouverture nasale selon la méthode déjà utilisée pour le calcul de la distance approximative entre l'extrémité apicale du processus cornual droit et l'extrémité apicale du processus cornual gauche. Face au foramen infra-orbitaire droit et à l'extrémité caudale de l'os incisif la distance minimum du bord latéral de celui-ci au plan sagittal égale $50 \mathrm{~mm}$. A partir du bord médial du même os elle mesure $40 \mathrm{~mm}$. A ce niveau la largeur rostrale de la cavité nasale, pratiquement à son maximum, atteignait soit $100 \mathrm{~mm}$, soit $80 \mathrm{~mm}$ suivant la mesure pratiquée (bords rostraux des os nasaux ou contre parois internes). Chez le Bœuf la largeur de l'ouverture nasale se place plus en avant.

En vue nuchale (tableau 4, figure 3-B, pl. 2) épais, sont considérablement ébréchés et, du côté droit, des dégâts particulièrement importants ont atteint la crête mastoïdienne. Les mesures les concernant ne sont donc qu'approximatives (tabl. 4). En dépit de ces dommages, l'obliquité des crêtes nuchales et mastoïdiennes, ou ce qu'il en reste, se révèle modérée. De cette façon le rétrécissement vers le haut de la face nucale parait moins accentué qu'il ne l'est en général chez les bisons mâles actuels d'Amérique que nous avons examinés.

Ce qui frappe surtout à première vue ce sont les dimensions relativement faibles $d u$ foramen magnum et, dans une moindre mesure, celles des deux condyles. Ceux-ci, moins proéminents vers l'arrière que les crêtes nucales et mastoïdiennes, ne dépassent caudalement ni le renflement qui surmonte le trou occipital ni même le bord supérieur de ce dernier. Ils sont régulièrement arrondis dans le sens de leur petit diamètre.

En vue ventrale (tableau 4, figure 3-C)

En ce qui concerne le basioccipital, en avant de l'extrémité rostrale du revers ventral de chaque condyle, se développe ventralement et latéralement un relief accusé épais dont le bord latéral diverge sensiblement de celui du relief symétrique. Ainsi la largeur du basioccipital mesurée au niveau de ces reliefs $(88,5 \mathrm{~mm}$ ) (fig. 3-C, 29) dépasse sensiblement la largeur minimum $(71 \mathrm{~mm})$ de la partie rostro-ventrale des condyles (fig. 3-C, 30) qu'ils limitent caudalement. Chez Bos, au contraire, en arrière de ces reliefs, le basioccipital s'élargit.

Quand on considère l'angle latéral de chaque condyle, on remarque qu'il comporte, de façon nette, un prolongement latéral ce qui selon McDonald (1981, pl. 25 et 26) s'observe surtout chez les bisons de grande taille. Longueur basale et condylo-basale ont pu être mesurées.

Les dents jugales supérieures (tableau 11)

88 M1 droite : Ses fossettes sont de dimensions réduites : l'usure, qui a presque atteint leur fond, a fait disparaître complètement la colonnette interlobaire linguale. La face occlusale est plane.

M2 droite: Les fossettes ont conservé chacune leurs deux diverticules mésial et distal mais la colonnette interlobaire est pratiquement arasée. La face masticatrice est presque plane : les cuspides sont très peu saillantes par rapport au sillon vestibulolingual médian.

PALEO, 15 | 2003 
90 M3 droite : On remarque la présence sur la face occlusale d'un îlot qui correspond à la partie vestibulaire isolée $d u$ repli d'émail séparant la colonnette interlobaire du protocône. Les cuspides sont nettement en relief.

91 Une P3 et une P4 supérieures droites portant l'indication de provenance A55, trouvées isolées mais sans doute à proximité du crâne, doivent-elles être attribuées au crâne A55-B1 ? L'usure avait presque atteint leur collet. Le diamètre vestibulo-lingual de P4 au niveau occlusal égal à $25 \mathrm{~mm}$ est légèrement inférieur à celui du lobe mésial de la M1 (26 mm). Selon Skinner et Kaisen (1947, p. 146, pl. 13) un bison d'Amérique actuel dont la denture parvient à un tel degré d'usure (entre les stades 3 et 4 de ces auteurs) a dépassé l'âge de la pleine maturité. En outre, la forme de la face occlusale des M1 et M2, aplanie, semblerait indiquer, d'après les observations de Haynes (1984), que le bison $n^{\circ} 2 A B$ se nourrissait de végétaux fortement abrasifs et que l'usure de ses molaires devait être relativement rapide.

\subsection{La mandibule droite A 83 (tableau 12, pl. 4))}

In situ elle se trouvait à moins de $5 \mathrm{~cm}$ de l'extrémité rostrale du calvarium A55-B1, légèrement plus haut sur le versant de l'éboulis. Sa région antérieure manque jusqu'au foramen mentonnier et, à l'opposé, l'angle mandibulaire est entamé. Ses dimensions, surtout celles qui permettent d'apprécier sa longueur et la hauteur de sa branche montante avant que les dommages n'intervinssent, sont largement supérieures aux dimensions correspondantes des mandibules B18 et B5 du bison $\mathrm{n}^{\circ} 1 \mathrm{AB}$. Cependant on constate avec surprise qu'en ce qui concerne la robustesse du corps (hauteur et épaisseur), du moins sa robustesse relative, c'est ce dernier, une femelle il est vrai âgée et trapue, qui prend “ l'avantage". Pourtant nous ne mettons pas en doute l'appartenance du calvarium A55-B1 et de la mandibule A83 au même individu car l'occlusion des deux séries antagonistes M1-M3 droites se réalise de la meilleure façon.

93 Le degré d'abrasion des molaires inférieures (le fond des fossettes de M1 est presque atteint) se place entre les stades 3 et 4 de Skinner et Kaisen (loc. cit., pl. 13). Il correspond à peu près à celui des dents d'un bison d'Amérique ayant dépassé de peu l'âge de la pleine maturité : conclusion en accord avec celle résultant de l'examen des molaires supérieures.

94 La saillie des cuspides par rapport au sillon qui sépare les deux mésiales des distales est à peu près nulle sur $M 1$, légèrement accusée sur $M 2$, surtout du côté lingual, et assez nette sur M3.

\subsubsection{Les vertèbres et côtes}

\subsection{Vertèbres}

Ont peut-être appartenu au bison $2 \mathrm{AB}$ :

Cervicales : C4 (A25), C6 (A27)

Thoraciques : T2 (B49), T3 (B32), T4 ou T5 (A52), T7 (B22), T8 ou T9 (A89)

Une vertèbre thoracique, A98, représentée sur le plan 5 du secteur A presque en contact avec A89, était parfaitement disposée parallèlement à la vertèbre A89. Elle n'a pas jusqu'ici été retrouvée. Les vertèbres B49, B32, B22, ont été découvertes à proximité du métacarpe B56 attribué au bison $n^{\circ} 2$ AB. Tous ces os ont leurs épiphyses soudées. 


\subsection{Côtes} partie orale du crâne A55-B1 tandis que sa région ventrale supportait l'extrémité proximale du grand radius A20. Sa hauteur, du point le plus bas de la glène jusqu'au sommet de l'épine, mesure $540 \mathrm{~mm}$, valeur supérieure à celles données par Stampfli (1963), Degerbol et Iversen (1945), Millot (1945) pour des bisons d'Europe actuels. Les dimensions de la cavité glénoïde sont plus grandes que celles de l'omoplate du bison $\mathrm{n}^{\circ} 1 \mathrm{AB}$, en particulier son diamètre antéro-postérieur. On remarque l'infléchissement modéré du bord latéral ; en cela la différence avec ce que l'on remarque le plus souvent chez les Bos n'est pas évidente. Cependant cette face articulaire apparaît proportionnellement moins large que chez ces derniers.

\subsubsection{Les os des membres antérieurs}

\subsection{L'humérus droit A111 (tableau 14)}

101 A peu près réduit à sa moitié distale. Recueilli à proximité de la mandibule A83, de la scapula A22 et des vertèbres thoraciques A89 et A98.

\subsection{L'humérus gauche B2 (tableau 14, pl. 5)}

102 Ne figure pas sur le plan. Sa région proximale est détériorée vers l'avant mais on peut, sans gros risque d'erreur, mesurer sa longueur, de son extrémité distale au sommet de la tête articulaire (fig. 6-A) après avoir déterminé l'emplacement de celui-ci au prix d'une reconstitution partielle.

Dans leurs parties comparables les deux humérus ont les mêmes dimensions et présentent les mêmes caractéristiques anatomiques.

Région distale

crâniale, la saillie distale de la lèvre latérale de la trochlée est relativement peu accusée ; la gorge qui la sépare du capitulum est peu encaissée. En vue médiale, l'axe de l'os étant vertical, le bord distal apparaît à peu près rectiligne, trochlée et condyle médial se situant à peu près au même niveau (fig. 6-B). En vue latérale, la dépression du capitulum est bien développée de bas en haut dans le sens crânio-caudal (fig. 14-1). En vue caudale, le bord latéral de la fosse olécranienne est franchement arqué mais son bord médial l'est moins.

106 L'humérus $\mathrm{B} 2$ se distingue des humérus du bison femelle $\mathrm{n}^{\circ} 1 \mathrm{AB}$ à la fois par ses dimensions beaucoup plus fortes et son indice de robustesse encore plus élevé. 


\subsection{Le radio-ulnaire droit A20-B31 (tableaux 15 et 16, pl. 5)}

107 Le radius A20, auquel adhérait une partie de l'ulna, gisait à moins de $20 \mathrm{~cm}$ de la portion A55 du crâne et à $10 \mathrm{~cm}$ environ de l'humérus droit A111. La partie manquante de l'ulna se trouvait dans le secteur B, en contact avec le métacarpe gauche B35 du même individu. Elle a retrouvé sa place de façon à reconstituer le radio-ulnaire A20-B31 qui s'articule avec l'humérus A111.

\subsection{Le radio-ulnaire gauche B4 (tableaux 15 et 16)}

Il ne figure pas sur le plan. De toute évidence il appartenait au même sujet que le radioulnaire A20-B31.

Dans les deux cas (B4 et A20-B31), radius et ulna, indépendants l'un de l'autre au-dessus de l'espace interosseux proximal, sont intimement soudés au-dessous de celui-ci, le bord latéral et le bord médial de la diaphyse de l'ulna demeurant bien distincts, surtout son bord latéral séparé du radius par une fente relativement profonde le long du $1 / 5^{\text {ème }}$ distal de celui-ci. Comme chez le bison $\mathrm{n}^{\circ} 1 \mathrm{AB}$ l'axe principal de l'olécrâne fait avec celui du radius un angle moins ouvert que chez Bos, le sommet de l'olécrâne étant plus nettement renversé vers l'arrière.

\subsection{Les radius (tableau 15)}

Région proximale (figure 15):

110 L'échancrure du bord palmaire en contact avec les facettes articulaires de l'ulna est large mais peu profonde : en direction dorsale elle ne dépasse pas le bord palmaire de la petite cavité glénoïde. Elle forme un angle légèrement supérieur à $90^{\circ}$ qui répond bien à l'angle saillant, quelque peu obtus, réalisé par les deux facettes de l'ulna. Le relief latéral d'insertion, comme chez la femelle $n^{\circ} 1 \mathrm{AB}$, est de type Bison mais on note une très faible tendance de l'aire de fixation du ligament collatéral médial du coude à apparaître du côté dorsal. Quant au bord antérieur de la face articulaire, il se montre " ondulé " en vue dorsale (fig. 15-3).

Région distale :

On y retrouve les caractères signalés sur les radius A11 et A37 du bison $n^{\circ} 1$.

Remarques:

- La dépression longitudinale dorsale pour l'extenseur radial du carpe est très peu accentuée mais le relief qui la limite latéralement est épais et assez proéminent.

- En vue distale on constate que la face articulaire du radius est séparée de celle de l'ulna par une rainure courte et très peu profonde.

\subsection{Les os du carpe (tableaux 17 à 21)}

Plusieurs éléments du carpe droit (le pyramidal A102, le semi-lunaire A103, l'unciforme A103 et le capitato-trapézoïde A100 ont été trouvés bien groupés, au-dessous de la partie proximale du radius droit A20. Ne manquent que le pisiforme et le scaphoïde. Des carpiens ont été mis au jour dans le secteur B ainsi que le radio-ulnaire B4 et le métacarpe B56 correspondant. Il s'agit du pyramidal et du capitato-trapézoïde B46 et du scaphoïde B24. 
113 En vue latérale le bord externe de la face en contact avec l'os crochu (unciforme) décrit une concavité relativement prononcée et étroite ce qui est, habituellement, considéré comme caractéristique du Bison mais, en même temps, le bord proximal de la région proximo-dorsale qui s'articule avec le radius est régulièrement arrondi, ce qui n'est pas précisément reconnu comme une disposition propre à ce genre.

\subsection{Le semi-lunaire droit A103 (tableau 18)}

La concavité du bord latéral de la face dorsale n'apparaît que légèrement accusée ; l'os se révèle plus large que haut.

\subsection{Le scaphoïde gauche B24 (tableau 19, figure 16-6)}

115 Il présente des dispositions considérées par Stampfli (1963, p.138, fig. 18) comme caractéristiques du Bison. En vue médiale, le bord proximal de la face articulaire répondant au processus styloïde du radius dessine une concavité relativement étroite et profonde tandis que le bord dorsal est légèrement convexe. D'après Slott-Moller (1988, p. 44), il convient d'observer les trois facettes articulaires (a , b, c) en relation avec le capitato-trapézoïde. Chez le Bison elles sont bien distinctes et la plus vaste, $b$, présente tous les caractères d'une glène alors que chez le Bœuf " elle constitue une large gouttière oblique dans le sens latéral ". Nous retrouvons sur le scaphoïde B24 d'Habarra les caractères “ Bison " signalés par cet auteur. Signalons enfin que, conformément à ce qu'a noté Bibikova (1958) à propos du même genre, chez le mâle $\mathrm{n}^{\circ} 2$ d'Habarra, comme d'ailleurs chez la femelle $n^{\circ} 3$ présentée plus bas, la hauteur dorsale du scaphoïde surpasse nettement celle du semi-lunaire (tabl. 19 et 18).

\subsection{L'unciforme (hamatum, os crochu) droit A103 (tableau 20, figure 16-11)}

116 En vue proximale, la crête qui sépare la facette articulaire en contact avec le pyramidal de la facette articulaire en contact avec le semi-lunaire, légèrement courbe dans son ensemble, est saillante, surtout dans ses deux tiers palmaires, plus saillante semble-t-il qu'elle ne l'est chez Bos. La zone articulaire touchant le semi-lunaire comporte une partie dorsale déprimée, $a$, et une partie palmaire, $b$, fortement proéminente, en forme de tronc de cône dont la base est bordée par la crête citée plus haut. Ce relief est plus accusé que chez le Bœuf.

\subsection{Les capitato-trapézoïdes A100 (droit) et B46 (gauche) (tableau 21, figure 16-8)}

117 En vue palmaire, ainsi que l'a constaté Slott-Moller (loc. cit., p. 48, pl. 10), chez le Bison la trochlée en relation avec le scaphoïde possède une gorge profonde, $a$, et une lèvre médiale très proéminente, ce qui ne semble pas être le cas chez le Bœuf.

\subsection{Les métacarpes (tableau 22) ; B56 (droit) (figure 17-1), B35 (gauche) (figure $17-6$; pl. 5)}

118 Ils ont été découverts à $70 \mathrm{~cm}$ l'un de l'autre, le premier auprès des mandibules B18 et B5 du bison $n^{\circ} 1$ mais un peu plus profondément enfouis, le second au contact de la portion d'ulna B31 du bison $\mathrm{n}^{\circ} 2$. Ces deux os sont plus longs en valeur absolue que les 
métacarpes du bison femelle $\mathrm{n}^{\circ} 1 \mathrm{AB}$ mais plus courts par rapport à leurs humérus et radius respectifs. Les deux bords de la région moyenne de leur diaphyse apparaissent à peu près parallèles en vue dorsale ou en vue palmaire. Comparés aux os canons antérieurs des autres bisons d'Habarra ils se révèlent particulièrement trapus : leur indice de robustesse égale 25,1 (B56) et 25,3 (B35).

Extrémité proximale (figure 17-6)

119 La face articulaire a un diamètre transversal relativement plus grand que celui des métacarpes de la femelle $\mathrm{n}^{\circ} 1 \mathrm{AB}$ tandis que tous les reliefs d'insertion sont nettement plus proéminents, aussi bien ceux de l'extenseur radial du carpe (face dorsale), de l'extenseur oblique du carpe (côté médio-palmaire) et du muscle ulnaire latéral (côté latéral) que ceux des tendons qui s'insèrent sur la face palmaire. A noter que ces derniers constituent un bourrelet inégalement épais mais pratiquement ininterrompu en arrière de la fossette synoviale, ce qui n'est pas le cas chez le bison femelle $n^{\circ} 1$ pourtant très âgée. D'autre part la dite fossette est nettement plus étroite chez le mâle $\mathrm{n}^{\circ} 2 \mathrm{AB}$.

\section{Région distale}

Les tubercules supra-articulaires latéraux sont globuleux et saillants mais pas plus que chez le bison $\mathrm{n}^{\circ} 1 \mathrm{AB}$; ils le seraient même proportionnellement un peu moins (tableau 22).

\subsection{Phalanges antérieures (tableaux 23, 24 et 37, figures 18 et 19)}

- 1ère phalange B30 (tabl. 23, fig. 18-2 et 3) et 1ère phalange B62 (réduite à sa moitié proximale) : Toutes deux s'articulent bien avec l'os canon droit B56. Si cette association est justifiée, B30 est la 1ère phalange latérale et B62 la 1ère phalange médiale (tabl. 23).

-1ère phalange : B60

- 2èmes phalanges antérieures (tableau 24) : A85 (fig. 19-1,2,3) et A101

-2èmes phalanges (antérieures ?) : B62, B13

- 3èmes phalanges antérieures (tableau 37) : A96 et B65.

\subsubsection{Les os des membres postérieurs}

\subsection{Le fémur droit A114 et le fémur gauche A33 (tableau 26 ; pl. 6)}

Comme les tibias et les métatarses, in situ ils se trouvaient plus haut sur la pente de l'éboulis que les grands os longs des membres antérieurs, que la mandibule A83 et la portion crânienne A55, à une distance de $1,50 \mathrm{~m}$ environ de celle-ci. Le grand trochanter des deux os est détruit et les lèvres de leur trochlée ont été rongées : les dents d'un carnassier y ont laissé leur empreinte, de façon particulièrement nette sur le fémur gauche. Le condyle latéral du fémur droit, trouvé à part, fortement endommagé, n'a pu retrouver sa place. Bien que la tête articulaire elle-même ne soit pas intacte nous donnons, tableau 26, avec risque d'erreur probablement assez faible, la longueur mesurée du sommet de cette face articulaire à la base médiale de la trochlée.

\subsection{Le tibia droit A29 et le tibia gauche A49-124 (tableau 27, figure 20 ; pl. 6)}

Le tibia droit est en parfait état de conservation; le tibia gauche a été découvert en deux morceaux: l'un, A49, correspond à la moitié distale de l'os plus une partie 
proximale de la diaphyse ; l'autre, A124, à la moitié latérale de la région proximale. Les deux portions s'ajustent sans difficulté. Ils gisaient à $50 \mathrm{~cm}$ l'un de l'autre, le gauche, A49-124, presque en contact avec le fémur gauche A33.

En vue crâniale, au-dessous du condyle médial, le bord de la diaphyse décrit de haut en bas d'abord une convexité assez accusée dont le maximum se place vers le milieu de l'os puis une concavité, également prononcée, dont le maximum se situe à $85 \mathrm{~mm}$ environ de l'extrémité distale. Cette disposition s'observe aussi chez le bison femelle $n^{\circ} 1 \mathrm{AB}$ mais de façon moins nette. Le tibia du mâle $n^{\circ} 2 A B$ apparaît plus franchement “ sinueux ". En ce qui concerne le bord latéral, plutôt concave vers le haut, il tend à devenir rectiligne sur un peu moins de la moitié distale.

Région proximale

Il n'existe pas de vestige du péroné comparable en forme et en dimensions à celui que l'on voit fréquemment sous le condyle latéral des tibias de Bos. En vue latérale, le bord supérieur du condyle externe est fortement convexe, ce qui correspond à ce qu'observe Olsen (1960, p. 12).

\section{La face caudale (fig. 20-2) (A29 droit)}

'extrémité proximale des crêtes d'insertion $\mathrm{n}^{\circ} 1$ et $\mathrm{n}^{\circ} 2$ se situe à peu de distance du plateau tibial mais leur extrémité distale, en position médiane sur la diaphyse, atteint à peine le quart inférieur de l'os. La crête $n^{\circ} 2$ est particulièrement vigoureuse dans sa partie inférieure. La crête $\mathrm{n}^{\circ} 3$, très courte et peu saillante, rejoint le bord médial de la diaphyse légèrement au-dessous de la moitié supérieure de l'os. Quant à la crête $\mathrm{n}^{\circ} 4$ qui nous intéresse particulièrement, son extrémité proximale s'implante un peu plus bas que les crêtes $n^{\circ} 1$ et 2 . Encore plus courte, relativement, que la même crête du tibia A15 appartenant au bison $n^{\circ} 1 \mathrm{AB}$, elle rejoint distalement le bord médial de la diaphyse, très largement au-dessus de la moitié inférieure de l'os, contrairement à ce que nous avons observé sur les tibias de B. bison des collections de l'Institut de Préhistoire et Géologie du Quaternaire de l'Université Bordeaux I mais de façon conforme à ce que l'on voit habituellement chez Bos (cf. fig. 21). Toutes ces crêtes d'insertions musculaires sont à la fois moins épaisses et moins saillantes que celles qui ornent la face caudale du bison $n^{\circ} 1 \mathrm{AB}$ plus âgé.

\section{La face articulaire distale}

126 Comme chez le bison $n^{\circ} 1 \mathrm{AB}$, les deux facettes latérales répondant à l'os malléolaire sont complètement séparées et non décalées l'une par rapport à l'autre comme elles le sont chez le Bœuf d'après Stampfli (1963).

\subsection{L'os malléolaire droit A63 (tableau 29)}

En vue latérale, son bord dorsal est convexe comme il l'est selon Stampfli (loc. cit.) chez les bisons tandis que les deux facettes proximales touchant les deux facettes correspondantes du tibia se situent au même niveau (autre caractère "Bison ").

\subsection{Les os du tarse}

Talus droit A67 et talus gauche A116 (tableau 32)

Calcanéum droit A46 et calcanéum gauche A88 (tableau 33)

Naviculo-cuboïde droit A53 et naviculo-cuboïde gauche A117 (tableau 30) 
Grand cunéiforme droit A62 (tableau 31, figure 16-13 et 14)

Tous sont caractéristiques du Bison. Précisions que sur les deux naviculo-cuboïdes, la tubérosité du cuboïde n'est que modérément développée et que le relief articulaire proximal, sur lequel vient buter le bord distal de la lèvre latérale de la trochlée plantaire du talus lors des mouvements d'extension, épouse la forme d'un arc de cercle. Chez le Bœuf ce relief décrit généralement un angle droit (Stampfli, 1963, fig. 26). Enfin, conformément à ce qu'à observé Brugal (1983, p.364) chez les bisons, le grand cunéiforme ne forme pas un angle médio-dorsal comme chez les bœufs mais plutôt un bord médio-dorsal régulièrement arrondi.

\subsection{Les métatarses A47 droit, B35 gauche (tableau 34 ; pl. 6)}

Le métatarse droit, seul bien conservé, surpasse sensiblement en longueur (290 mm) le plus grand des 295 os canons postérieurs de Bison actuel d'Amérique (276 mm) mesurés par McDonald (1981, tabl. 30, p. 97). Sa robustesse n'est pas moins remarquable. Le rapport centésimal de la largeur au milieu de l'os à la longueur totale atteint 16,55, valeur relativement élevée aussi bien chez B. priscus que chez B. bison. Si l'on examine la face proximale, on constate que le bord médial de la facette latérale en contact avec le naviculo-cuboïde touche le bord latéral de la facette médiale en contact avec le petit cunéiforme ce qui pour Olsen (1960, p. 10) est caractéristique du Bison.

\subsection{Les phalanges postérieures (tableaux 35 et 36)}

1ère phalange postérieure latérale A54 (fig. 18-6 et 7) et 1ère phalange postérieure médiale A60. Elles s'articulent avec le métatarse droit A47.

1ère phalange postérieure latérale A122 et 1ère phalange postérieure médiale A119. Elles s'articulent avec le métatarse gauche A115.

2ème phalange postérieure latérale A87 (fig. 19-7, 8 et 9). Elle s'articule avec la 1ère phalange A54.

2ème phalange postérieure médiale A95. Elle s'articule avec la 1ère phalange A60.

2ème phalange postérieure latérale A120. Elle s'articule avec la 1ère phalange A122.

2ème phalange postérieure médiale A121. Elle s'articule avec la 1ère phalange A119.

Les phalanges A122, A119, 120 et 121 se trouvaient groupées, en gisement, à proximité du métatarse gauche A115.

\subsubsection{Le sujet $n^{\circ} 2 A B$, conclusions}

erait difficile, sinon impossible, de découvrir dans un des troupeaux de bisons vivant aujourd'hui en Europe ou en Amérique un individu aussi grand que celui dont nous venons de décrire les principaux éléments du squelette. Pour s'en convaincre il suffit de consulter les tableaux 1 et 2 et 8 à 10 tout en regrettant l'insuffisance des données relatives à B.bonasus. En revanche notre bison fossile pyrénéen ne soutient pas de la même façon la comparaison avec les bisons de son espèce (B. priscus) plus anciens, encore plus imposants, comme ceux de Châtillon-Saint-Jean (Drôme) étudiés par Mourer-Chauviré (1972) ou de Mosbach en Rhénanie (Schertz, 1936) pour ne s'en tenir qu'au seul continent européen (tabl. 22 et 34). 
La tête osseuse du mâle d'Habarra présente les principales caractéristiques de B. priscus, notamment une ampleur considérable des processus cornuaux. Le mieux conservé de ces appendices, à forte courbure principale, ne montre pas à son extrémité distale, malheureusement non intacte, l'ébauche de torsion vers l'arrière fréquente chez $B$. bonasus et $B$. bison. Rappelons aussi que si l'on considère la forme de la section le long de la cheville osseuse frontale, on est conduit à diriger la corne proprement dite vers l'avant, sans préjuger de la dernière direction prise par celle-ci.

Par sa forme générale le crâne se révèle plus proche de celui des bisons d'Europe actuels que de celui des bisons d'Amérique actuels ou sub-actuels. Les séries de $B$. bonasus sur lesquelles nous fondons notre appréciation sont celles étudiées par Empel (Roskoss et Empel, 1961). Cet auteur (p. 63, tabl. 2) a mesuré la longueur basale (mais pas la longueur totale) et la largeur maximum au niveau des orbites de 41 têtes osseuses (22 femelles et 19 mâles). D'après ses chiffres nous avons évalué l'indice de la deuxième de ces dimensions par rapport à la première. Chez les mâles il varie de 56,3 à 70,2, la moyenne égale 64,4. Le même indice chez le "taureau " d'Habarra est très proche de cette moyenne : 63,4 .

En ce qui concerne B. bison, ne disposant pas d'un nombre suffisant de spécimens dont nous connaissons la longueur basale, la largeur maximum aux orbites a été estimée en fonction de la longueur totale. D'après nos propres mesures cet indice atteint :

- 55,6 chez le bison $n^{\circ} 2 \mathrm{AB}$ d'Habarra (tabl. 3),

- respectivement 56,9,62,2 et 55,9 chez 3 sujets mâles d'Europe actuels (tabl. 8),

- respectivement 59,5, 57,1 et 61,6 chez 3 sujets mâles d'Amérique actuels (tabl. 10).

- McDonald (1981, tabl. 29) a établi les valeurs moyennes suivantes, notamment pour les mâles de la forme B. bison bison :

- largeur maximum aux orbites $324,6 \pm 1,2 \mathrm{~mm}(\mathrm{n}=117)$

- longueur maximum : 535,3 $\pm 2,3 \mathrm{~mm}(\mathrm{n}=56)$.

Le rapport centésimal évalué à partir de ces deux moyennes égalerait 60,6 et serait donc assez peu différent du rapport correspondant chez le bison $n^{\circ} 2$ d'Habarra.

La silhouette d'un bison, comme d'ailleurs celle de tout autre quadrupède, est pour une bonne part conditionnée par la forme de la ligne dorsale qui relie les processus épineux du rachis et Poplin (1984) a montré que B. priscus, B. bonasus et B. bison présentent chacun un profil dorsal caractéristique. Malheureusement, en ce qui concerne le bison $\mathrm{n}^{\circ} 2 \mathrm{AB} \mathrm{d}^{\prime} H a b a r r a$ les rares vertèbres découvertes à ce jour et leur état de conservation ne nous fournissent aucune indication à ce sujet.

Il reste que, la plupart des grands os longs du bison $\mathrm{n}^{\circ} 2$ étant souvent en bon état de conservation, nous pouvons évaluer les rapports de longueur entre ceux-ci et tenter des comparaisons avec les deux espèces actuelles dans l'espoir de mettre en évidence des ressemblances et/ou des différences. Pour cela nous reprendrons certains graphiques utilisés lors de la détermination du sexe: figures 9 à 13. Le mode de réalisation de leur tracé a déjà été indiqué dans le chapitre consacré à ce problème.

La figure 10 montre d'indéniables analogies entre les 3 bisons mâles d'Europe et le sujet fossile d'Habarra qui ne se singularise qu'en raison de sa plus haute stature. Les segments correspondants des quatre tracés ne sont certes pas rigoureusement parallèles entre eux mais il s'en faut de peu. Nous soulignerons encore la brièveté des métapodes. 
149 Les diagrammes des figures 10 et 12 font apparaître, au contraire, d'incontestables dissemblances entre les bisons mâles actuels d'Amérique d'une part et, d'autre part, les bisons mâles de l'espèce bonasus et le bison $n^{\circ} 2 A B$ d'Habarra. En effet, chez les premiers, les métatarses sont proportionnellement plus allongés par rapport au tibia et au fémur comme le sont les métacarpes par rapport aux humérus et radius. Au total il n'est pas impossible que malgré des métatarses plus longs la croupe soit relativement plus basse chez B. bison bison.

150 Ces diverses conclusions, résultats d'observations limitées, ne sont peut-être que provisoires : elles attendent d'être confirmées, réfutées ou nuancées. Ce qui semble peu contestable c'est la puissance de l'avant-train du bison d'Habarra : la forme trapue des métacarpes sur laquelle nous avons insisté en témoigne. Le caractère massif de ce sujet est d'autre part révélé par la comparaison de ses talus avec ceux d'autres bisons de la même espèce (fig. 27).

\subsubsection{Bison $n^{\circ} 3 B$ femelle}

La mandibule gauche B88 (tableau $12 ;$ pl. 4)

Planche 4 - Bison priscus Habarra. Mandibules, face latérale, de quatre individus : 3 femelles ( $1 A B$, $3 \mathrm{~B}, 4 \mathrm{E})$ et 1 male (2AB).

Plate 4 - Bison priscus Habarra. The lateral face of the mandibles of four individuals: 3 females (1AB, $3 B$, $4 E)$ and 1 male (2AB).

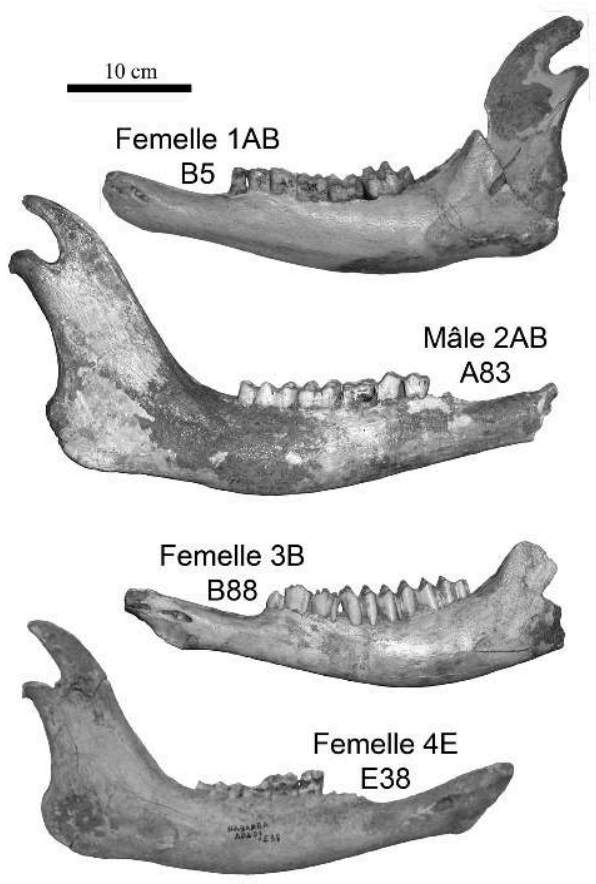

151 Elle est incomplète du fait de la destruction de sa branche montante et de sa région angulaire. Les dents jugales sont présentes mais pas les incisives et la canine. Les quelques mensurations praticables indiquent un animal à la fois moins grand et moins lourd que les sujets $n^{\circ} 1 \mathrm{AB}$ femelle et, à plus forte raison, $\mathrm{n}^{\circ} 2 \mathrm{AB}$ mâle. Il s'agit donc vraisemblablement d'une femelle. 
Le degré d'évolution de la dentition et l'usure des prémolaires et molaires indiquent que ce bison n'avait pas atteint le stade 2 de la maturité selon Skinner et Kaisen (loc. cit. p. 146, pl. 13). La M3 avait terminé son éruption mais son lobe distal est à peine entamé par l'abrasion, ce qui correspondrait au groupe $n^{\circ} 5,4-6$ ans de la classification de Reher (1974) établie par cet auteur lors de ses recherches sur des sites d'abattage de bisons paléoindiens. Le sommet de l'entostyle de M1 “ communique " avec la partie vestibulaire de la couronne tandis que celui de M2, bien qu'un peu usé, reste indépendant, du moins son apex. Quant à celui de M3, il reste distant de la table d'usure.

Les cuspides des trois molaires sont aiguës et saillantes par rapport au sillon vestibulolingual qui sépare les deux mésiales des deux distales. Cela ne surprend guère chez un individu relativement jeune, même dans l'hypothèse où il consommait des plantes abrasives.

L'attribution au même animal du semi-lunaire gauche A4, plus petit que le semi-lunaire du bison $n^{\circ} 1 A B$ (tabl. 18), peut être envisagée.

\subsection{Secteur C, gisement amont (fouilles, relevés et déterminations in situ A. Clot)}

Ce secteur a surtout livré des ossements de Rhinocéros et de Renne. Cependant le Bison y était aussi représenté, notamment par une vertèbre thoracique (3 ${ }^{\text {ème }}$ ou adjacente), $\mathrm{C} 6$, dont nous donnons ci-dessous quelques dimensions. Une $2^{\text {ème }}$ phalange $\mathrm{C} 18$ et des sésamoïdes C19 n'ont pas été retrouvés.

Vertèbre thoracique $\mathrm{C6}$ :

- Longueur approximative du corps : $79 \mathrm{~mm}$,

- Largeur au niveau du processus articulaire caudal : $46 \mathrm{~mm}$,

- Largeur de la face articulaire caudale : $97 \mathrm{~mm}$.

Le processus épineux est fracturé à $47 \mathrm{~mm}$ au-dessus du processus articulaire caudal.

\subsection{Secteur E, gisement amont (fouilles, relevés et déterminations in situ F. Delpech)}

\subsubsection{Bison $\mathrm{n}^{\circ}$ 4E femelle, associé au Rhinocéros Coelodonta antiquitatis}

\subsubsection{Situation dans le gisement. Identification}

Les os du bison $\mathrm{n}^{\circ} 4 \mathrm{E}$ étaient plus ou moins éparpillés à l'intérieur d'un rectangle long de 2,50 $\mathrm{m}$ et large de 1,50 m environ. Les pièces les plus lourdes, comme le fémur E2, le tibia E1 et le crâne E12, gisaient un peu plus bas que les autres le long de la pente, mêlées aux ossements du bison $n^{\circ} 5 \mathrm{E}$ (fig. 24).

En gisement il n'existait pas vraiment d'os en connexion anatomique mais quelques éléments d'une même patte restaient encore en contact, ou presque, les uns avec les autres. C'est le cas du métatarse E73 et de ses premières phalanges E73a et E73b. De même, la pièce la plus proche de l'axis E75 était l'atlas E70. Autre fait remarquable, les sept vertèbres cervicales et les deux premières thoraciques, dispersées in situ, s'ajustent en bon ordre. 
La majorité des ossements du Bison $\mathrm{n}^{\circ} 4 \mathrm{E}$ provient de la partie la plus haute du secteur alors que la plupart de ceux du bison $\mathrm{n}^{\circ} 5 \mathrm{E}$ ont été mis au jour dans la partie la plus basse.

Il s'agit bien des restes d'un bison femelle. L'examen du crâne, dont les résultats sont exposés plus bas, l'atteste ainsi que le rapport des longueurs: métacarpe-humérus, métacarpe-radius, métatarse-fémur et métatarse-tibia (tabl. 1 ; fig. 13).

Exemples :

- indice métacarpo-radial $=65,2$

- indice métatarso-tibial $=65,3$.

Le sujet $\mathrm{n}^{\circ} 4 \mathrm{E}$, contrairement au sujet $\mathrm{n}^{\circ} 5 \mathrm{E}$, avait atteint un âge extrêmement avancé.

De tous les squelettes de bisons d'Habarra, celui du bison $\mathrm{n}^{\circ} 4 \mathrm{E}$ est le mieux conservé, en ce sens qu'il compte moins de parties manquantes que les autres.

\subsubsection{Le crâne E12 (tableaux 3, 4 et 5 ; pl. 1, 2 et 3)}

Il a été découvert en contrebas par rapport à la plupart des autres pièces appartenant au bison $\mathrm{n}^{\circ} 4 \mathrm{E}$ : à $1,80 \mathrm{~m}$ de la mandibule gauche E73, à 1,50 $\mathrm{m}$ de la mandibule droite E38 et à $2 \mathrm{~m}$ de l'atlas E70. Or ses condyles s'adaptent idéalement à la cavité crâniale de cette vertèbre tandis que ses molaires droites, les seules bien dégagées, s'engrènent convenablement avec celles de la mâchoire inférieure antagoniste.

Non entièrement reconstitué, il se présente en plusieurs parties. La plus volumineuse comprend :

- les os frontaux, incomplets, d'où se détachent les processus cornuaux, l'un, le gauche, très bien conservé, l'autre, réduit à sa région proximale sur une longueur de $11 \mathrm{~cm}$,

- les os nasaux dont il ne reste qu'une portion caudale longue de $10 \mathrm{~cm}$,

- le pariétal,

- le temporal droit et une portion du temporal gauche.

Une autre partie correspond au maxillaire supérieur gauche auquel adhèrent notamment le lacrymal et le jugal, l'un et l'autre incomplets. Le maxillaire supérieur droit a entraîné une partie du palatin correspondant. Chaque maxillaire est recouvert d'une couche de calcite si épaisse, en particulier sur sa face externe, qu'il a perdu presque totalement son galbe. A tout cela il faut ajouter l'os incisif droit isolé et certainement quelques petits fragments indéterminés.

Les os frontaux et le pariétal

7 En avant des processus cornuaux, au niveau desquels ils sont légèrement surélevés, les os frontaux, détériorés latéralement, se révèlent à peu près plats. En arrière de ces appendices et en avant de la saillie nucale, le profil du pariétal apparaît légèrement concave. Les deux lignes de suture fronto-pariétales, aux fines dentelures toujours visibles, qui convergent vers la suture interfrontale forment chacune un petit bourrelet qui, probablement, trahit aussi le grand âge atteint par le bison $\mathrm{n}^{\circ} 4 \mathrm{E}$.

8 Les sillons supra-orbitaires sont peu marqués et chaque foramen supra-orbitaire se place au milieu de l'espace compris entre le bord caudal de l'orbite et la cheville osseuse. Ce que l'on voit de la partie antérieure du frontal gauche prouve que les orbites, sensiblement moins proéminentes que sur le crâne du bison mâle $n^{\circ} 2$, étaient aussi relativement plus proches des processus cornuaux. 
Les processus cornuaux (tableaux 5 et 8 à 10 ; figure $25 ;$ pl. 1,2 et 3 )

“Au départ" en amorçant leur mouvement de latéralité, ils se dirigent vers l'arrière et le haut, non vers l'arrière et le bas comme ceux du crâne E55-B1 et réalisent un angle caudal d'environ $59^{\circ}$ (moins ouvert que chez le bison $n^{\circ} 2$ ) par rapport au plan sagittal. Au cours de leur " ascension ", déjà perceptible au niveau du col, ils dépassent quelque peu caudalement le plan de l'occipital et ne se recourbent qu'à peine vers le plan sagittal. Les points les plus ventraux de l'arc supérieur se situent plus haut que les os frontaux. C'est naturellement le processus gauche qui se prête le mieux à un examen détaillé.

170 A environ $13 \mathrm{~cm}$ du bord dorsal de la couronne il semble qu'une légère torsion s'ébauche : en effet la cannelure principale caudale qui prend naissance à $6 \mathrm{~cm}$ environ de la base du processus vient se placer peu à peu en position dorso-caudale. Toutefois cette disposition ne tend pas de façon très évidente à amener l'apex vers l'avant. Cependant, la torsion qui, très fréquemment, chez B. bison et B. bonasus aboutit à orienter la région apicale vers l'arrière n'existe pas.

$171 \mathrm{Au}$ niveau de la couronne, le diamètre dorso-ventral dépasse modérément le diamètre antéro-postérieur. Mais, toutes proportions gardées, l'écart entre ces deux dimensions, mesurées tous les $5 \mathrm{~cm}$, s'accentue jusqu'à l'extrémité libre, de même que la profondeur des cannelures qui parcourent la face caudale et la face ventrale. Voir figure 25 l'évolution (dans le même sens) de la forme du pourtour de la cheville.

A la faveur d'une écornure ancienne on constate que la région apicale du processus cornual gauche est creuse jusqu'au bout.

173 La distance séparant l'apex de la cheville osseuse droite de celui de la cheville osseuse gauche devait atteindre entre 620 et $640 \mathrm{~mm}$ (dimension évaluée suivant le procédé utilisé dans le cas du crâne E55-B1). Même si l'on ne retient que la plus basse de ces valeurs, l'envergure des cornes du bison femelle $\mathrm{n}^{\circ} 4 \mathrm{E}$ d'Habarra reste supérieure :

- à celle des cornes des femelles du Bison d'Amérique actuel (B. bison) (tabl. 9),

- à ce qu'elle est en moyenne chez les mâles de cette dernière espèce (tabl. 10),

- à ce qu'elle était, peut-être, chez les femelles de Bison priscus (tabl. 6).

174 En revanche, même si l'on ne retient que la valeur la plus forte, $640 \mathrm{~mm}$, force est d'admettre qu'une telle envergure demeure très inférieure à celle des cornes des mâles adultes de l'espèce fossile (tabl. 6).

Maxillaires supérieurs et palatins (tableaux 3 et 4 )

175 Le maxillaire supérieur et le palatin droits sont incomplets. M2 et M3 supérieures, toujours en place, face occlusale dégagée, sont usées et la longueur (ou hauteur) du métacône de cette dernière dent n'atteint que $15,5 \mathrm{~mm}$. Le maxillaire supérieur gauche est encore en connexion avec le palatin, le zygomatique, le lacrymal, une petite partie du frontal, du nasal et de l'os incisif. M2 et M3 disparaissent sous la calcite, de même ( ?) que d'hypothétiques vestiges de prémolaires. M1 est absente.

Face nucale et face ventrale (tableau $4 ;$ pl. 2)

176 La comparaison du crâne E12 avec le crâne A55-B1 permet de mettre en évidence des différences importantes, non seulement en ce qui concerne les dimensions, presque toujours à l'avantage du second (tabl. 4). En effet, chez le premier les crêtes bordant dorsalement et latéralement l'occipital se révèlent considérablement moins épaisses et moins saillantes. Elles tendent à dessiner avec la ligne joignant les processus mastoïdes 
un trapèze isocèle à petite base relativement moins courte, les crêtes nucales étant moins fortement obliques de bas en haut dans le sens latéro-médial. La crête latérale droite, bien conservée, dont la face caudale n'est que légèrement bombée, se trouve en retrait par rapport à la protubérance nucale. Au niveau du contact occipital-temporal, elle s'élargit en développant un angle médial épais qui ne semble pas exister chez Bos. D'autre part elle ne prend guère la forme d'une crête aiguë, comme cela se voit sur des crânes de Bison actuel et, sans doute, de B. priscus (quand ils ne sont pas détériorés).

De chaque côté d'un foramen magnum plus vaste, même en valeur absolue, que celui du grand spécimen A55-B1, les condyles, arrondis dans le sens de leur petit diamètre, font saillie en arrière de la protubérance nucale et du plan de l'occipital. Ce n'est pas le cas chez le bison mâle ${ }^{\circ} 2 \mathrm{AB}$.

En vue ventrale, on constate que l'angle latéral de chaque condyle développe un prolongement rostro-latéral un peu moins prononcé que chez le bison $\mathrm{n}^{\circ} 2 \mathrm{AB}$. Quant au relief situé en avant de chacun des condyles s'il est moins vigoureux que chez le mâle $n^{\circ} 2$, il l'est plus que chez le Bœuf. Au niveau de ces reliefs (fig. 2, 29) l'occipital apparaît sensiblement plus large qu'au niveau du rétrécissement de la zone articulaire ventrale (fig. 2, 30), ce qui constitue une différence sensible avec ce que l'on constate chez Bos (tabl. 4). En revanche celui-ci possède des tubercules musculaires beaucoup plus proéminents.

Les dents jugales supérieures (tableau 11)

179 Ne sont guère observables que M2 droite (assez mal) et la M3 adjacente. Elles se révèlent usées à tel point que la hauteur du paracône de $\mathrm{M} 3$ n'égale que $16,5 \mathrm{~mm}$ et celle du métacône 15,5 $\mathrm{mm}$ ainsi que nous l'avons signalé précédemment. Le diamètre mésio-distal de M2 mesure approximativement $26 \mathrm{~mm}$ et celui de M3 $32 \mathrm{~mm}$. Toutefois l'abrasion n'avait pas encore dépassé le fond des fossettes de ces dents. L'usure semble être allée au-delà du stade " Old Age " tel qu'il est représenté par Skinner et Kaisen (1947, pl. 11, 5c).

La mandibule droite E38 (tableau $12 ;$ pl. 4)

180 A quelques exceptions près, ses dimensions diffèrent peu de celles des mandibules B18 et $\mathrm{B} 5 \mathrm{du}$ bison femelle $\mathrm{n}^{\circ} 1 \mathrm{AB}$. En bon état de conservation, y compris dans la région alvéolaire de son extrémité rostrale, elle porte 6 dents jugales mais a perdu ses incisives et sa canine. Seul le bord caudal de la tête du processus condylaire est légèrement endommagé. Le processus coronoïde, intact, est un peu moins long que celui de la mandibule $\mathrm{B} 5 \mathrm{du}$ bison $\mathrm{n}^{\circ} 1 \mathrm{AB}$ : il ne dépasse pas vers l'arrière le bord du processus angulaire.

181 La dépression longitudinale qui parcourt la face latérale du corps, au-dessous des prémolaires, de $\mathrm{M} 1$ et de $\mathrm{M} 2$, se situe probablement au niveau de la région apicale des racines de ces dents. Sa présence est sans doute liée à l'âge avancé du sujet. Le bord antérieur de la symphyse est fortement oblique de haut en bas dans le sens rostrocaudal.

La mandibule gauche E73 (tableau 12)

182 Moins bien conservée que la précédente. La partie antérieure comprenant la région incisive et la région d'implantation des $\mathrm{P} 2$ et $\mathrm{P} 3$ se trouve encastrée dans un bloc stalagmitique. Elle s'ajuste néanmoins assez bien dans sa partie libre avec le reste de la mandibule. Sous P2 et P3 le corps est partiellement détruit latéralement. Si le condyle 
est intact, l'extrémité du processus coronoïde est brisée. 4 dents seulement sont observables : P4, M1, M2 et M3.

Les dents jugales inférieures

Elles aussi témoignent de l'âge très avancé du sujet. Les dessins de leur face occlusale ont disparu. Le lobe distal de M3, lui-même, est arasé et la racine qui lui correspond a été entamée par l'action de la dent antagoniste. Une exception : la $2^{\text {ème }}$ prémolaire n'est usée qu'à son sommet. Quoi qu'il en soit, l'usure dentaire avait atteint et sans doute dépassé le stade le plus avancé représenté par Skinner et Kaisen (loc. cit. p. 146, pl. 13, 5).

\subsubsection{Vertèbres et côtes}

Les côtes, à l'état de fragments, n'ont pu être identifiées avec la précision souhaitable. Les vertèbres font l'objet d'une étude particulière de la part de $\mathrm{N}$. Cancel.

Vertèbres cervicales (tabl. 39 et 40) : Atlas E70 ; C3 E73 ; C4 E73 ; C5 E56 ; C6 E32 ; C7 E31

Vertèbres thoraciques (tabl. 40) : T1 E30; T2 E7 ; T8 ou T9 E44 ; T12 E43 ; T13 E35.

Vertèbres lombaires (tabl. 40) : L1 E50 ; L2 E41 ; L4 E40 ; L5 E65

Sacrum (tabl. 41). : E15

\subsubsection{La scapula gauche E9 (tableau 13)}

Bien conservée dans son ensemble, elle gisait à moins d'un centimètre du crâne E12. Les mesures prises dans sa région ventrale diffèrent peu des mesures correspondantes relevées sur la scapula $\mathrm{B} 6$ du bison femelle $\mathrm{n}^{\circ} 1 \mathrm{AB}$.

En vue latérale, au-dessus de la cavité glénoïde, on remarque la forte convexité du bord caudal du col qui présente des reliefs d'insertion accentués. Ceux-ci indiquent un âge avancé: l'hypothèse d'une attribution de cette pièce au bison $\mathrm{n}^{\circ} 4 \mathrm{E}$ s'en trouve confortée. Cependant le cartilage scapulaire n'était pas ossifié : il n'en subsiste aucune trace le long du bord dorsal. Cela peut surprendre puisque ce cartilage est minéralisé chez la femelle $\mathrm{n}^{\circ} 1 \mathrm{AB}$ moins âgée que la femelle $\mathrm{n}^{\circ} 4 \mathrm{E}$ si l'on en juge d'après le degré d'usure dentaire. Mais, selon Barone (1986, p. 453), chez le Bœuf, le bord dorsal de l'omoplate "se laisse souvent envahir par l'ossification chez les vieux sujets" ce qui semble signifier que ce n'est pas toujours le cas.

191 Il existe des concrétions dans la partie supérieure de la fosse infra-épineuse, au sommet de l'épine scapulaire et sur le bord crânial de la fosse supra-épineuse.

192 La concavité de la glène s'accorde avec le galbe de la tête articulaire de l'humérus gauche E39 recueilli à 1,30 $\mathrm{m}$ de la scapula E9. L'infléchissement modéré mais net du bord latéral de cette cavité est bien caractéristique du Bison.

193 Dans sa région moyenne, la face médiale est largement et profondément déprimée dans le sens longitudinal. Si le tubercule sus-glénoïdal se révèle assez peu proéminent, le processus coracoïde est bien individualisé.

\subsubsection{Les os des membres antérieurs}

6.3.1.5.1 L'humérus droit E46 et l'humérus gauche E39 (tableau 14, figure 14-2; pl. 5) 
Planche 5 : Bison priscus Habarra. Os longs du membre antérieur de deux individus : 1 mâle (2AB) et 1 femelle (4E).

Plate 5: Bison priscus Habarra: Long bones of the forelimb of two individuals: 1 male (2AB) and one female (4E).

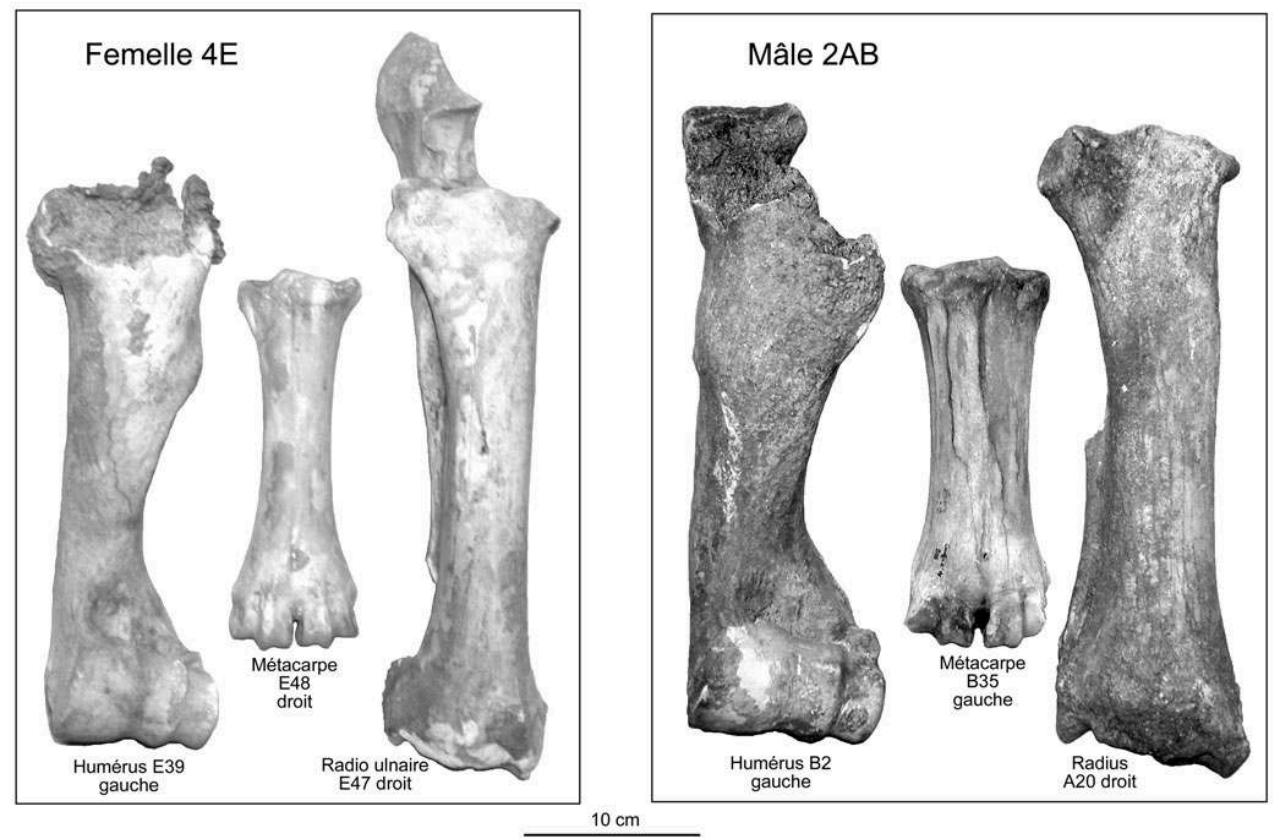

Comparé à la femelle, le mâle a des os plus robustes et un métacarpe plus court relativement aux humérus et radius.

Compared to the female, the bones of the male are more robust; in addition, the male metacarpal is shorter relative to the humerus and radius.

L'humérus droit E46 est réduit à une portion distale. Des fragments de sa diaphyse ont été envoyés pour datation ; nous n'en avons pas les résultats.

L'humérus gauche E39 est plus long que l'humérus A32 de la femelle $\mathrm{n}^{\circ} 1 \mathrm{AB}$ et aussi moins massif mais ses dimensions n'atteignent pas, il s'en faut de beaucoup, celles de l'humérus $\mathrm{B} 2$ du bison mâle $\mathrm{n}^{\circ} 2 \mathrm{AB}$. On remarque également la vigueur accusée des reliefs d'insertions musculaires, notamment de la ligne tricipitale et de la tubérosité deltoïdienne. Cette disposition est manifestement en relation avec le grand âge du sujet. On y observe les caractères "Bison " déjà signalés à propos des individus $\mathrm{n}^{\circ} 1 \mathrm{AB}$ et $n^{\circ} 2 \mathrm{AB}$, notamment l'allongement dans le sens crânio-caudal et de bas en haut de la dépression latérale du capitulum. L'extrémité proximale comporte quelques traces de morsures.

\subsection{Le radio-ulnaire droit E47 (tableaux 15 et 16, figure 15-2 ; pl. 5)}

Pratiquement complet. Les deux os de l'avant-bras présentent tous les caractères génériques déjà signalés chez les bisons $1 \mathrm{AB}$ et $2 \mathrm{AB}$. Il existe bien, par exemple, en vue dorsale une nette sinuosité du bord antérieur proximal du radius.

Le radio-ulnaire $\mathrm{E} 47$, plus long que les radio-ulnaires du bison $\mathrm{n}^{\circ} 1$ mais plus court que ceux du bison $n^{\circ} 2$, se distingue des uns et des autres par sa robustesse moins forte.

A l'extrémité proximale, la plage de fixation du ligament collatéral du coude ne montre qu'une légère tendance à s'orienter dorsalement, comme sur les radius déjà décrits. En fait, en vue antérieure, la zone d'insertion est à peine perceptible (fig. 15-2). La coulisse 
dorsale pour l'extenseur radial du carpe se trouve assez nettement délimitée, sa crête latérale (épaisse) et sa crête médiale (aiguë) étant fortement saillante en raison du grand âge de l'individu.

\subsection{Le radius gauche E45 (figure 24)}

Symétrique du radius E47, il a été « sacrifié » pour datation ${ }^{14} \mathrm{C}$ et daté de $14500 \pm 260$ ans BP (Gif-7106).

\subsection{Les os du carpe (tableaux 17 à 21)}

\subsection{Le pyramidal droit E73 et le pyramidal gauche (tableaux 17, figure 16-2)}

De même que chez les sujets $n^{\circ} 1 \mathrm{AB}$ et $\mathrm{n}^{\circ} 2 \mathrm{AB}$, le sommet de la région proximo-dorsale est arrondi vers le haut mais plus modérément. Quant au bord latéral de la zone en contact avec l'os crochu, franchement concave, il indique le genre Bison sans équivoque.

\subsection{Le semi-lunaire droit E73 (tableau 18, figure 16-4)}

Des quatre semi-lunaires de Bison recueillis à Habarra, c'est le seul qui soit plus haut que large. Le rapport centésimal de la hauteur de la face dorsale au diamètre transversal maximum égale 104,5, valeur plus fréquemment rencontrée chez Bos que chez Bison. Cependant la partie dorsale de sa face articulaire proximale est moins bombée que sur les autres spécimens du même gisement ce qui constituerait un caractère Bison mieux affirmé.

\subsection{Le scaphoïde droit E73 (tableau 19, figure 16-7)}

En vue médiale, son bord dorsal est rectiligne, légèrement oblique de haut en bas dans le sens dorso-palmaire, non convexe comme celui du scaphoïde du bison $n^{\circ} 2 \mathrm{AB}$. L'examen de la face articulaire distale (fig. 16-7) permet de constater, conformément à ce qu'a déjà signalé Slott-Moller (1988, p. 454) à propos d'autres bisons, que les trois facettes $(a, b, c)$ en appui sur le capitato-trapézoïde sont distinctes, celle se situant en position médio-palmaire (b), la plus étendue, ayant la forme d'une cavité glénoïde. D'autre part, la hauteur dorsale du scaphoïde dépasse celle du semi-lunaire, disposition propre au Bison.

\subsection{L'unciforme droit E73 (tableau 20, figure 16-12)}

La face articulaire qui répond au semi-lunaire comporte, mais de façon un peu moins nette que sur l'unciforme A103 (bison $n^{\circ} 2 \mathrm{AB}$ ), une zone dorsale relativement déprimée (a) et une partie palmaire saillante de forme tronconique (b).

\subsection{Le capitato-trapézoïde droit E73 (tableau 21, figure 16-9)}

En vue palmaire, on remarque que la lèvre médiale de la face articulaire en rapport avec le scaphoïde est relativement proéminente et la gorge qui la limite latéralement, profonde. Ce caractère, propre au bison (comparé au Bœuf), se révèle bien prononcé chez le sujet $n^{\circ} 4$ E d'Habarra. 
6.3.1.5.5 Les métacarpes (tableau 22), métacarpe droit $\mathrm{E48}$ (pl.5), métacarpe gauche B1 (figure 17-2 et 7)

Ils sont plus longs, même en valeur absolue que ceux du bison mâle $n^{\circ} 2 \mathrm{AB}$. Aucun des 276 canons antérieurs de Bison actuel d'Amérique (B. bison), sexes confondus, dont McDonald (1981, tabl. 30, p. 97) donne pour cette dimension, entre autres, l'intervalle de variation, n'est aussi grand. On constate aussi que leur diaphyse est assez nettement rétrécie vers le milieu de l'os et leur indice de robustesse sensiblement inférieur à celui du métacarpe $\mathrm{A} 38$ de la femelle $\mathrm{n}^{\circ} 1 \mathrm{AB}$. Cependant leur morphologie demeure tout à fait caractéristique du Bison. Par exemple on remarque le fort développement des tubercules distaux supra-articulaires.

206 La fossette synoviale de la face articulaire proximale s'ouvre sur la face palmaire entre les deux reliefs d'insertion, le plus médial de ceux-ci étant encore plus proéminent, mais moins étendu, que chez le mâle ${ }^{\circ} 2 \mathrm{AB}$ (fig. 17-6 et 7-a).

\subsection{Phalanges antérieures}

207 Aucune, à ce jour, n’a semble-t-il été découverte.

6.3.1.6 Les os des membres postérieurs

6.3.1.6.1 Fémur droit E2 (tableau 26 ; pl. 6) 
Planche 6 - Bison priscus Habarra. Os longs du membre postérieur de deux individus : 1 mâle (2AB) et 1 femelle (4E).

Plate 6 - Bison priscus Habarra: Long bones of the hindlimb of two individuals: 1 male (2AB) and 1 female (4E).

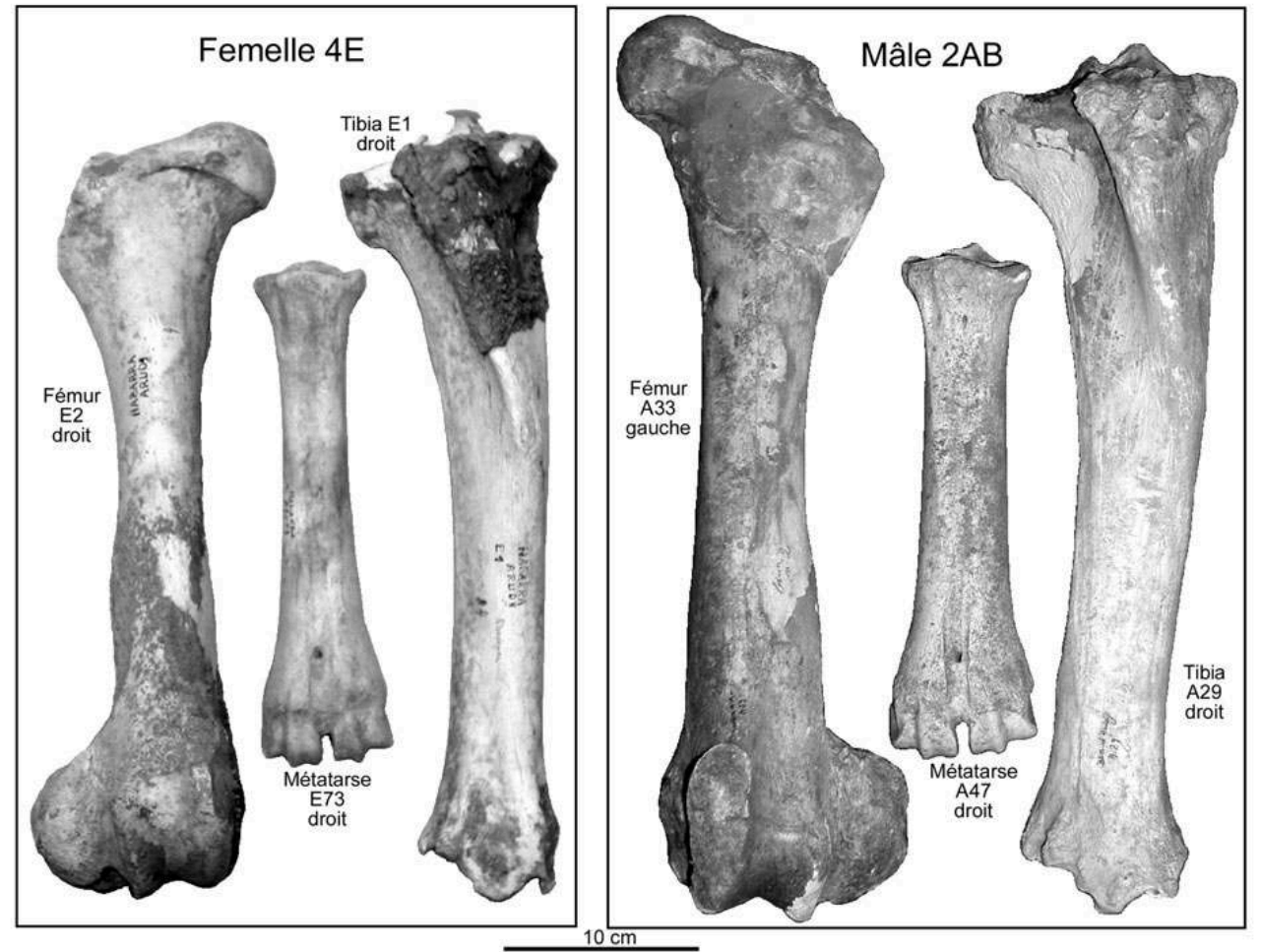

Comparé à la femelle, le mâle a des os plus robustes et un métatarse plus court relativement aux fémur et tibia.

Compared to the female, the bones of the male are more robust; in addition, the male metacarpal is shorter relative to the femur and tibia.

En gisement, son extrémité proximale, endommagée, surtout du côté latéral, était presque en contact avec le crâne $\mathrm{E} 12$ tandis que sa région distale reposait à moins de 15 $\mathrm{cm}$ de la face articulaire supérieure du tibia appartenant à la même patte.

\subsection{Le tibia droit E1 (tableau 27, figure 20 ; pl. 6)}

Son extrémité distale se trouvait relativement éloignée du métatarse droit E73, à environ $1,15 \mathrm{~m}$.

La face caudale (fig. 20-3)

210 La crête d'insertion $n^{\circ} 1$, dans sa partie proximale, se dédouble au niveau du trou nourricier. Plus bas, elle se prolonge par une ligne peu vigoureuse. La crête $\mathrm{n}^{\circ} 2$ prend naissance relativement haut, comme la crête $\mathrm{n}^{\circ} 1$. Extrêmement épaisse et proéminente, nettement oblique de haut en bas dans le sens latéro-médial, elle s'interrompt un peu au-dessous de la moitié proximale. En ce lieu elle parait comme " supplée " distalement par une crête également saillante, parallèle à la crête $n^{\circ} 1$ mais un peu plus proche que celle-ci du bord médial de la diaphyse. La crête $n^{\circ} 3$ est courte et beaucoup plus discrète que les crêtes $n^{\circ} 2$ et $n^{\circ} 4$ qui l'encadrent et lui sont parallèles. La crête $n^{\circ} 4$, peu allongée mais relativement puissante, disposée comme sur le tibia des bisons $n^{\circ} 1$ et $n^{\circ} 2$ d'Habarra, atteint le bord médial de l'os bien plus haut que le milieu de celui-ci. 


\section{Extrémité distale}

Les deux facettes articulaires répondant à l'os malléolaire sont complètement séparées l'une de l'autre et non décalées l'une par rapport à l'autre.

\subsection{L'os malléolaire gauche E25 (tableau 29)}

\subsection{Les os du tarse (tableaux 30 à 33)}

Talus droit E62 et talus gauche E37 (tabl. 32)

Calcanéum droit E74 et calcanéum gauche E37 (tabl. 33)

Cubo-naviculaire droit E73 et cubo-naviculaire gauche E37 (tabl. 30)

Grand cunéiforme droit E73 (tabl. 31)

Leur morphologie, typique du Bison, n'appelle pas de commentaires.

\subsection{Les métatarses E73 droit, E36 gauche (tableau 34 ; pl. 6)}

Parallèlement à ce que nous avons constaté à propos des canons antérieurs nous notons que ces deux os sont :

- relativement longs, sans doute plus longs que les plus grands des os canons des Bisons actuels, B. bonasus et B. bison tant mâles que femelles;

- moins massifs que ceux des bisons d'Habarra $n^{\circ} 2$ et $n^{\circ} 1$.

Les deux facettes caudales du plateau articulaire se trouvent séparées l'une de l'autre.

\subsection{Phalanges postérieures (tableaux 35 et 36, figures 18 et 19)}

$1^{\text {ère }}$ phalange médiale E73a - patte droite (tabl. 35)

$1^{\text {ère }}$ phalange latérale E73b - patte droite (tabl. 35 , fig. 18 -10 et 11)

$1^{\text {ère }}$ phalange E37 - patte gauche- sa région distale manque (tabl. 35)

$2^{\text {ème }}$ phalange médiale E73a - patte droite (tabl. 36, fig. 19 - 12, 13 et 14)

$2^{2 \mathrm{ème}}$ phalange latérale E73b - patte droite (tabl. 36)

$3^{\text {ème }}$ phalange E37, incomplète (endommagée vers l'avant)

\subsection{Sésamoïdes}

Grand sésamoïde axial E37 (tabl. 38)

Grand sésamoïde abaxial E37 (tabl. 38)

En gisement les phalanges E73 se trouvaient au contact du métatarse droit E73, les phalanges et les sésamoïdes E37 au contact du métatarse gauche E36.

\subsubsection{Le sujet $n^{\circ} 4 E$, conclusions}

2 Dans le troupeau des femelles qui l'accompagnaient la bisonne $n^{\circ} 4 \mathrm{E}$ se distinguait sûrement par sa taille relativement élancée. Si nous ignorons quel rôle elle jouait au sein de ce groupe, nous savons qu'elle avait atteint un âge vénérable.

Plus longues que celles des bisons actuels de même sexe (tabl. 5, 7 et 9) ses cornes étaient loin de posséder l'envergure du mâle $n^{\circ} 2 \mathrm{AB}$. Tout en s'orientant latéralement, 
la cheville osseuse gauche, seule entièrement conservée, se dresse dès le départ, au niveau du col. En même temps elle se dirige légèrement vers l'arrière puis, en s'élevant, décrit une courbe peu accentuée à convexité latérale. Lorsqu'on l'examine perpendiculairement à sa face latérale, son apex n'apparaît nettement incliné ni vers l'avant ni vers l'arrière. On se souvient que chez le " taureau " $n^{\circ} 2 A B$ les processus cornuaux s'affaissent dès leur naissance et que les points les plus ventraux de la grande concavité médio-dorsale dépassent vers le bas le plan des frontaux (pl. 2 et 3).

Skinner et Kaisen (1947) représentent en vue nucale des crânes de bisons mâles (pl. 17, 19 et 21) et femelles (pl. 23) appartenant à la forme américaine B. crassicornis Richardson, 1854 que McDonald $(1981$, p. 113) rattache à B. priscus. Ces documents montrent que les différences dans le mode d'érection des cornes, dans l'ensemble, sont les mêmes que celles constatées entre le mâle $\mathrm{n}^{\circ} 2 \mathrm{AB}$ et la femelle $4 \mathrm{E}$ d'Habarra. On voit sur quelques uns des spécimens figurés planche 23 par ces auteurs que le caractère propre aux femelles n'est pas toujours net. En effet, sur l'un d'eux (pl. 23, 7A) la concavité médiale des appendices cornuaux, au départ, descend au-dessous du plan des os frontaux. Cette disposition devait probablement apparaître aussi parfois chez des femelles de Bison des steppes européens.

Les rapports de longueur des principaux os des membres sont-ils les mêmes chez le bison $n^{\circ} 4 \mathrm{E}$ d'Habarra que chez les femelles des espèces B. bison et B. bonasus ? Notre documentation ne permet pas de répondre de façon sûre à cette question. En fait nous nous bornons à quelques constatations portant sur un trop petit nombre de sujets. Peut-être convient-il aussi d'évoquer le degré de précision des mesures quelque peu variable malgré toute l'attention portée à ces opérations. L'évaluation de la longueur d'un métacarpe, d'un métatarse et d'un tibia n'est-elle pas plus aisément reproductible de façon satisfaisante que celle de la longueur d'un humérus et d'un fémur prise au sommet de la tête articulaire (parfois plus ou moins endommagée) ou même d'un radius en connexion anatomique avec l'ulna?

Comme nous l'avons fait à propos des comparaisons du bison $n^{\circ} 2 \mathrm{AB}$ avec les bisons mâles des espèces bonasus et priscus, nous réutiliserons des diagrammes déjà tracés en vue de la distinction mâles-femelles (fig. 9 et 11).

Bison femelle $\mathrm{n}^{\circ} 4 \mathrm{E}$ et B. bison femelles (fig. 11)

Chez la bisonne d'Habarra,

- le métatarse serait plus court par rapport au fémur et au tibia ;

- le métacarpe serait plus court par rapport au fémur et au tibia.

- et, comparé à celui des sujets BM-1865-12-8-26 et BM-1850-11-22-102, le métacarpe serait plus court par rapport au radius; sur ce dernier point, il n'y a pas de différence sensible entre le sujet fossile et le bison actuel MP-1898-261.

Bison femelle $\mathrm{n}^{\circ} 4 \mathrm{E}$ et B. bonasus femelles (fig. 9)

En ce qui concerne les os du membre antérieur, les tracés présentent une certaine incohérence. Nous ignorons si cela tient à une variabilité particulière de la longueur relative de ces éléments du squelette ou à des problèmes de mesure déjà évoqués. Quoi qu'il en soit nous sommes contraints à une prudente réserve. En revanche nous devons souligner une dissemblance tout à fait remarquable touchant le membre postérieur: chez le Bison d'Europe actuel le métatarse est plus court par rapport au tibia.

Bison femelle $\mathrm{n}^{\circ} 4 \mathrm{E}$ et bison femelle $\mathrm{n}^{\circ} 1 \mathrm{AB}$ (fig. 13 et tabl.1) 
La femelle $1 \mathrm{AB}$ aurait un radius proportionnellement moins long par rapport à l'humérus et un métacarpe un peu moins court par rapport au radius. Cependant le rapport métatarse-tibia est à peu près le même chez les deux individus $(65,6$ et 65,3$)$. Il est plus fort que chez les 5 femelles " bonasus" ( rapport qui varie de 59,55 à 63,08) mais un peu plus faible que chez les trois femelles de B. bison (de 66,5 à 68,3) auxquelles nous les comparons.

\subsubsection{Bison $n^{\circ} 5 E$ femelle}

\subsubsection{Situation dans le gisement. Identification}

De cet individu nous ne possédons que quelques éléments des pattes postérieures et des vertèbres qui reposaient dans l'aire de répartition des ossements du bison $\mathrm{n}^{\circ} 4 \mathrm{E}$ mais, pour la plupart, dans la partie la plus basse de ce secteur. Ils se rapportent donc à une femelle (indice métatarso-tibial $=66,3$ ), un peu moins grande que la bisonne $\mathrm{n}^{\circ} 4 \mathrm{E}$, moins âgée aussi comme le prouve le stade d'ossification des différentes pièces : fémur (?), tibia, tarsiens et métatarsiens, vertèbres à épiphyses absentes ou imparfaitement soudées.

\subsubsection{Les restes du squelette}

\subsection{Vertèbres thoraciques}

T5 E73 ; T7 E20 ; T8 ou T9 E51 ; T11 ou T12 E61 ; T13 E58 ; T14 E53.

\subsection{Vertèbres lombaires}

L1 ou L2 E73 ; L3 E14 ; L4 E59.

\subsection{Fémur “E sous bloc" (tableau 26)}

Nous considérons son appartenance au bison $\mathrm{n}^{\circ} 5 \mathrm{E}$ seulement comme probable. Il proviendrait du secteur E mais n'a pas été découvert au cours des fouilles systématiques. Il ne figure donc pas sur le plan. Il est réduit à une portion distale. La ligne épiphysaire, encore apparente, tendait à disparaitre : elle est d'ailleurs effacée par endroits. Chez le Bison d'Europe, d'après Koch (1934), la soudure de l'épiphyse distale intervient au cours de la deuxième moitié de la $5^{\text {ème }}$ année.

En vue crâniale, il est surprenant de constater que le bord médial et le bord latéral de la trochlée ne convergent pas de façon régulière vers le bas comme cela se voit le plus souvent chez le Bison (fig. 7c). En effet, l'obliquité latéro-médiale de la lèvre trochléenne latérale s'accentue assez brusquement vers le bas de telle sorte que ce fémur eut été peut-être rapporté au genre Bos (fig. $7 \mathrm{~b}$ ) dans un autre contexte.

Ses dimensions sont compatibles avec celles du tibia droit E23, des tarsiens énumérés plus bas, des métatarses E16 (dr.) et E13 (g.), autant de pièces qui, pour chaque côté, permettent des mises en connexion pertinentes. Toutefois si l'ajustement en position anatomique du fémur "E sous bloc" au tibia E23 ne soulève pas d'objections il ne prouve pas de façon indiscutable que les deux os appartenaient au même individu, l'union des faces articulaires étant ici moins intime que dans le cas d'une connexion tibia-talus ou même grand cunéiforme-métatarse. 


\subsection{Le tibia droit E23 (tableau 27, figure 20)}

Il est un peu plus long que les tibias du bison $n^{\circ} 1 A B$ mais légèrement moins grand que le tibia $\mathrm{E} 1 \mathrm{du}$ bison $4 \mathrm{E}$. La ligne épiphysaire proximale, en voie de disparition au moment de la mort de l'animal, demeure encore perceptible, en particulier du côté médial. Or la soudure du plateau tibial à la diaphyse s'opère vers le milieu de la $5^{\text {ème }}$ année chez le Bison d'Europe actuel (Koch, loc. cit.). On ne peut donc s'empêcher de penser que le degré d'ossification du tibia E23 s'accorde assez bien avec celui du fémur “E sous bloc ", ce qui donne force à l'idée que les deux os se rapportent probablement au même sujet.

\section{Région proximale}

En vue externe, le bord supérieur du condyle latéral décrit une forte convexité (caractère "Bison ").

Face caudale (fig. 20-4)

En raison de l'âge peu avancé du bison, les lignes d'insertion sont peu prononcées mais on constate la brièveté et la forte obliquité de la crête $n^{\circ} 4$ qui, comme chez les autres individus représentés à Habarra, atteint le bord médial de la diaphyse beaucoup plus haut que le milieu de l'os.

\subsection{Les os du tarse (tableaux 30, 32 et 33)}

Talus droit E21 et talus gauche E19 (tabl. 32)

Calcanéum droit E16 (tabl. 33)et calcanéum gauche E22

Cubo-naviculaire droit E17 (tabl. 30)

Grand cunéiforme droit E16 et grand cunéiforme gauche E10 (tabl. 31)

La morphologie de ces os indique sans ambiguïté le genre Bison. On note que la longueur des deux calcanéums dépasse légèrement celle des calcanéums du bison $n^{\circ} 4$ pourtant un peu plus grand que le bison $n^{\circ} 5$.

La ligne de suture de l'épiphyse des calcanéums est décelable surtout sur la face plantaire.

\subsection{Les métatarses E16 droit, E13 gauche (tableau 34)}

Un peu moins longs et à peine moins sveltes que ceux du bison $\mathrm{n}^{\circ} 4 \mathrm{E}$. Les facettes caudales articulaires proximales du métatarse E13, qui ne se touchent pas, sont néanmoins proches l'une de l'autre. Sur le métatarse E16, la partie correspondante de l'os étant endommagée, l'observation ne peut être faite.

\subsection{Phalanges postérieures (tableaux 35, 36 et 37, figure 19)}

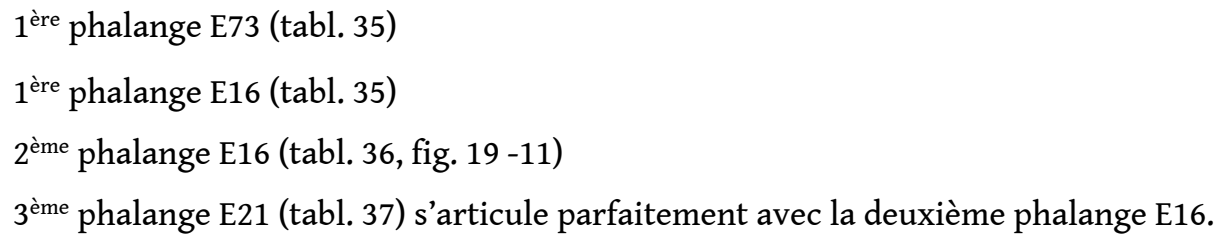




\subsubsection{Le sujet 5E, conclusions}

La figure 13 montre que dans le cas du bison $\mathrm{n}^{\circ} 5 \mathrm{E}$ le rapport de la longueur métatarsetibia est tout à fait comparable à ce qu'il est chez les deux autres femelles d'Habarra $\mathrm{n}^{\circ} 1 \mathrm{AB}$ et $4 \mathrm{E}$, donc différent de ce qu'il est chez les bisons mâles y compris le sujet fossile d'Habarra $n^{\circ} 2 \mathrm{AB}$ (fig. 13) et chez les bisons femelles d'Europe (fig. 9). La dissemblance avec les bisons femelles d'Amérique dont l'os canon postérieur est peutêtre encore moins court par rapport au tibia paraît évidente.

\subsubsection{La scapula droite E55}

Elle ne peut être attribuée ni au bison $n^{\circ} 1 \mathrm{AB}$, ni aux bisons $\mathrm{n}^{\circ} 4 \mathrm{E}$ et $\mathrm{n}^{\circ} 5 \mathrm{E}$, en raison des dimensions trop fortes de sa partie conservée (tabl. 13). Elle ne se rapporte pas davantage au bison $n^{\circ} 2 \mathrm{AB}$. Elle indique certes un sujet de grande taille mais l'insertion des muscles n'a laissé que de faibles traces ce qui dénote un âge moins avancé que celui atteint par le mâle $n^{\circ} 2 \mathrm{AB}$. Faut-il la rapprocher des restes du bison mâle $\mathrm{n}^{\circ} 10 \mathrm{du}$ secteur $\mathrm{D}$ dont il est question ultérieurement? A ce jour cette question restera sans réponse.

\subsection{Secteur F, gisement amont (fouilles et relevés G. Marsan et F. Certain)}

\subsection{Bison $\mathrm{n}^{\circ} 6 \mathrm{~F}$ femelle ( $=$ Bison $\mathrm{n}^{\circ} 5 \mathrm{E}$ femelle ?)}

258 Les ossements recueillis dans ce secteur gisaient non loin de ceux des secteurs D et E mais un peu plus bas sur la pente de l'éboulis (fig. 2). La plupart se rapportent au Renne mais deux portions crâniennes $\mathrm{F} 10$ et $\mathrm{F} 15$, deux vertèbres cervicales $\mathrm{F} 3$ et $\mathrm{F} 7$ et un coxal droit F2, qui étaient regroupés à l'intérieur d'un espace rectangulaire de $70 \mathrm{~cm}$ de long sur $40 \mathrm{~cm}$ de large, sont attribuables au Bison. Ces pièces, du moins les portions crâniennes sur lesquelles reposait un crâne de Renne, appartenaient sans doute au même individu.

\subsubsection{Le crâne F10-F15 (pl. 1, 2 et 3)}

\subsection{La portion crânienne F10 (tabl. 3, 4, 5)}

Elle est constituée en majeure partie des deux os frontaux, plus ou moins bien conservés, ornés chacun d'un processus cornual incomplet. Cet appendice dont la surface a presque perdu son caractère poreux mesure $135 \mathrm{~mm}$ du côté droit. Du côté gauche où il atteint $165 \mathrm{~mm}$ environ, sa morphologie autorisait une reconstitution et, partant, une évaluation de la distance entre les deux chevilles osseuses au niveau des apex selon le procédé déjà utilisé dans le cas des crânes E55-B1 et E12. Au-dessous des processus cornuaux la paroi des deux fosses temporales n'a guère subi de dégâts. De la crête nucale il ne reste environ qu'un tiers mais on voit bien que dans son ensemble elle ne devait être ni plus large ni plus saillante que chez la femelle ${ }^{\circ} 4 \mathrm{E}$.

Du côté gauche

L'os frontal concourt, avec l'extrémité caudale du lacrymal et l'os jugal, à la formation d'une orbite complète alors que du temporal adjacent ne subsistent, outre sa participation à l'édification de la fosse temporale, que le processus zygomatique et le 
tubercule articulaire. Le maxillaire supérieur, le prémaxillaire, l'os nasal et le palatin n'ont pas été découverts.

Du côté droit

Le frontal est fortement endommagé par suite d'une fracture ancienne ayant entraîné la destruction de l'os en avant d'une ligne joignant le bord postérieur de l'orbite à l'épine frontale. Du même côté il ne reste de vestige ni du lacrymal ni du nasal, ni du maxillaire ni du prémaxillaire. Cependant tout donne à penser que ces deux derniers os constituent la pièce F15 trouvée dans le même locus. L'os temporal comprend le processus articulaire, le tubercule articulaire fortement détérioré et une partie de l'écaille.

Les sillons supra-articulaires sont relativement accentués tant à droite qu'à gauche que sur les crânes déjà décrits (A55-B1 et E12).

L'occipital

Il n'en reste qu'un vestige rostral de sa partie basilaire : la cavité cérébrale se révèle donc largement ouverte.

Les quelques mesures effectuées sur le calvarium F10 diffèrent peu des dimensions correspondantes du spécimen E12 mais se situent bien en deçà de celles du crâne du bison mâle $n^{\circ} 2 \mathrm{AB}$. Il est clair que la pièce $\mathrm{F} 10$ représente une femelle.

Les os frontaux et les processus cornuaux

tes processus cornuaux, les os frontaux sont légèrement bombés de droite à gauche et dans le sens rostro-caudal, le maximum de cette convexité, modérée, se trouvant face à l'extrémité caudale des deux sillons supra-oculaires. Un peu plus en avant, en regard du bord dorsal des orbites, ils se creusent, formant une dépression proportionnellement plus accusée que celle signalée sur les crânes A55-B1 et E12.

266 La longueur de l'arc supérieur du processus cornual gauche reconstitué, évaluée à 261 $\mathrm{mm}$, se révèle légèrement plus courte que chez le bison femelle $\mathrm{n}^{\circ} 4 \mathrm{E}(270 \mathrm{~mm})$ tandis que l'envergure des cornes était probablement plus ample (tabl. 5) : entre 640 et 660 mm.

L'axe des chevilles osseuses réalise semble-t-il avec la suture frontale un angle caudal légèrement plus ouvert $\left(63^{\circ}\right.$ à gauche, $65^{\circ}$ à droite)( pl. 1) que sur le crâne E12 $\left(59^{\circ}\right)$ mais, comme chez ce dernier, chaque processus, tout en amorçant sa courbure latérale, se dirige simultanément vers l'arrière et le haut de telle façon que ses points les plus caudaux dépassent vers l'arrière le plan de l'occipital et que les points les plus ventraux de son arc supérieur se situent plus haut que le plan des os frontaux (pl. 3). A $130 \mathrm{~mm}$ environ du bord dorsal de la couronne on voit s'ébaucher une torsion dont on peut penser qu'elle dirigeait la région de l'apex vers l'avant.

chevilles osseuses du spécimen F10 ont donc bien des points communs avec celles du crâne E12 de même sexe. Mais leur comparaison révèle quelques différences, outre celles indiquées plus haut relatives aux dimensions. En effet, les processus cornuaux du bison $\mathrm{n}^{\circ} 6 \mathrm{~F}$ sont sensiblement moins dressés : ils réalisent avec le plan des os frontaux un angle latéral plus faible, d'où leur forme plus étalée (pl. 2). Leur section à peu près circulaire au niveau de la couronne, le reste à $10 \mathrm{~cm}$ de là et, semble-t-il, au niveau de la cassure (fig. 26). On remarque encore que l'appendice gauche présente sur ses faces ventrale et ventro-caudale des sillons longitudinaux moins profonds que les franches 
cannelures qui parcourent la cheville gauche du bison $n^{\circ} 4 \mathrm{E}$, ceci étant lié certainement à l'âge plus avancé de ce dernier.

Notons enfin que la cheville osseuse droite est creusée de sinus visibles au niveau de la cassure tandis que nous n'en observons pas au niveau de la fracture du processus gauche plus proche de l'apex.

\subsection{La portion crânienne F15 (tableaux 3, 4, 5 et 11)}

Il s'agit essentiellement d'un maxillaire supérieur droit encore en connexion avec le prémaxillaire et le palatin correspondants. Il porte la série des six dents jugales et, de même que le palatin, il s'est séparé de son homologue gauche (non retrouvé) suivant la ligne de suture médiane, la désarticulation s'étant produite sans opposition comme le prouve le très bon état des surfaces d'union avec l'os symétrique. Il y a de fortes chances pour que cette pièce ait appartenu au même animal que la pièce F10 à laquelle nous n'avons pu l'unir en raison de cassures ayant entraîné une importante perte de matière osseuse.

Le prémaxillaire

D'après Guthrie (1990, p. 182-184) les bisons d'Amérique actuels (B. bison), dont la nourriture est surtout constituée d'herbe rase, ont le mufle proportionnellement plus large que celui des bisons d'Europe qui s'alimentent de façon plus variée (graminées plus ou moins hautes et dicotylédones). A l'appui de cette remarque l'auteur représente (fig. 7-5) les prémaxillaires d'un bison d'Amérique et ceux, plus rétrécis vers l'avant, d'un bison d'Europe. En même temps il les compare aux prémaxillaires d'un bison des steppes (B. priscus) qui, en ce qui concerne leur largeur, occupent une position intermédiaire. Les contours du bord rostral du prémaxillaire droit F15 l'apparentent justement à ces derniers.

La rangée dentaire $P 2-M 3$

Le cément garnit la fossette des prémolaires et les deux fossettes des molaires, à l'exception de celle encore béante du lobe distal de M3 à peine atteint par l'usure. La pointe du métastyle de cette dent est encore intacte de même que celle de son entostyle. Un tel degré d'abrasion dentaire correspondrait à peu près au groupe 5 (4-6 ans) de Reher (1974) et au stade de la première maturité selon Skinner et Kaisen (1947). La face occlusale de M1 est presque plane tandis que celle de M2 et M3 possède des cuspides fortement saillantes.

\subsubsection{Le bison $n^{\circ} 6 \mathrm{~F}$ ou bison $n^{\circ} 5 E$}

Autant que l'on puisse en juger d'après leurs parties conservées, les crânes F10-15 et E12 étaient de dimensions, grosso modo, comparables. Comme les os du bison $n^{\circ} 5 \mathrm{E}$ (subadulte) sont à peu près du même ordre de grandeur que leurs homologues du bison $4 \mathrm{E}$ on en vient naturellement à penser que le calvarium F10-15 et les os des membres postérieurs du bison $n^{\circ} 5 \mathrm{E}$ qui indiquent, les uns et les autres, un individu sub-adulte ou adulte jeune, appartenaient peut-être au même animal. Nous n'en possédons pas aujourd'hui la preuve formelle mais nous sommes amenés à exprimer des réserves quant à l'existence d'un bison $\mathrm{n}^{\circ} 6$ distinct du bison $\mathrm{n}^{\circ} 5$.

274 D'autre part nous n'avons aucune raison sérieuse de rapprocher le maxillaire supérieur $\mathrm{F} 15$ de la mandibule gauche $\mathrm{B} 88$ du bison $\mathrm{n}^{\circ} 3 \mathrm{~B}$, plus petit que le bison $\mathrm{n}^{\circ} 1 \mathrm{AB}$ et à plus 
forte raison que le bison $n^{\circ} 5 \mathrm{E}$, d'autant plus que les reliefs des molaires du maxillaire droit F15 ne pourraient s'opposer correctement aux reliefs des molaires de la mandibule droite (non découverte) du bison $\mathrm{n}^{\circ} 3 \mathrm{~B}$ tels que nous permettent de les imaginer les dents de la mâchoire gauche B88.

Les dimensions de l'os coxal F2 figurent tableau 25. Les épiphyses des vertèbres F3 et F7, détachées du corps n'ont pas été retrouvées.

\subsection{Le secteur D, gisement amont (fouilles, relevés et déterminations in situ F. Delpech et J.-Ph. Rigaud)}

Outre des os de Bison le secteur D a livré des restes de Rhinocéros (Coelodonta antiquitatis), de Cheval (Equus caballus) et de Renne (Rangifer tarandus).

En l'état des découvertes cette partie de la caverne apparaît comme le lieu de rendezvous d'éléments assez hétérogènes qui, en ce qui concerne le Bison, n'autorisent que peu d'essais de mise en connexion. Plusieurs individus, qui diffèrent par l'âge et la taille, seulement représentés par quelques restes, y ont été plus ou moins clairement distingués mais il est relativement difficile de rassembler les ossements appartenant à chacun d'eux; les résultats de nos tentatives pour y parvenir ne sont pas toujours concluants.

278 Ces pièces reposaient sur la pente de l'éboulis, 4 mètres plus bas environ que celles des secteurs $\mathrm{A}$ et $\mathrm{C}$, non loin des os des bisons $4 \mathrm{E}$ et $5 \mathrm{E}$ qui se trouvaient légèrement plus au nord, en contrebas.

279 A ce jour rien ne donne à penser que certains des os qui manquent chez les individus des secteurs $\mathrm{A}, \mathrm{B}$, E et $\mathrm{F}$ se trouvent parmi ceux, déterminés, du secteur $\mathrm{D}$ : par exemple les vestiges des membres antérieurs du bison $5 \mathrm{E}$.

\subsubsection{Le bison $\mathrm{n}^{\circ}$ 7D femelle}

Nous lui rapportons un humérus droit, D1, dont la région proximale, en grande partie absente, pourrait avoir été rongée, et lui associons un radius gauche entier D7. Si les deux os, bien caractéristiques du genre, ne peuvent être placés en connexion anatomique, on remarque que chacun d'eux devait répondre parfaitement au symétrique de l'autre.

\subsubsection{Humérus D1 et radio-ulnaire D7 (tableaux 14 et 15)}

On ne voit ni sur l'humérus ni sur le radius la moindre trace de soudure (complètement réalisée) des épiphyses. Les dimensions de ces os indiquent un animal à la fois plus petit et plus gracile que les femelles $n^{\circ} 1 A B, n^{\circ} 4 \mathrm{E}$ et $n^{\circ} 5 \mathrm{E}$. La mandibule $\mathrm{B} 88$ (bison $n^{\circ} 3 \mathrm{~B}$ ) appartenait elle aussi à une femelle de faible stature peu trapue mais probablement plus jeune que le bison $\mathrm{n}^{\circ} 7 \mathrm{D}$.

De l'ulna ne subsiste que l'extrémité distale articulaire intimement soudée au radius. Au-dessus les deux os étaient indépendants: leur séparation s'est effectuée sans cassure comme le montre l'observation de la face palmaire du radius. Cela semble signifier que l'animal n'avait sans doute pas atteint un âge très avancé. 
6.5.1.2 Pièces du secteur ayant probablement appartenu au sujet $n^{\circ} 7 D$, du moins à un bison de taille comparable

\subsection{Le talus droit D48 et le calcanéum droit D86 (tabl. 32 et 33)}

Ils s'adaptent bien l'un à l'autre. L'astragale est plus petit en tous sens que les autres astragales de Bison d'Habarra.

\subsection{Le métatarse droit D59 et le métatarse gauche DB3 (tabl. 34)}

Relativement grêles, ils appartenaient à un même sujet que l'on est tenté de confondre avec le sujet 7D. Un peu plus grands que ceux de la femelle $n^{\circ} 1 \mathrm{~A}$, ils sont au contraire plus courts que ceux des femelles $4 \mathrm{E}$ et $5 \mathrm{E}$. Le rapport centésimal de leur longueur à celle du radius $\mathrm{D} 7$ entre dans le domaine de variation déterminé par des observations (trop peu nombreuses) portant sur des squelettes de bisons femelles d'Europe et d'Amérique.

\begin{tabular}{|l|l|}
\hline & Longueur métatarse/Longueur radius \\
\hline Bison n'7D d'Habarra & \\
\hline D59 dr/D7 & 81,3 \\
\hline DB3 g/D7 & 80,6 \\
\hline Bisons actuels (cf. tabl. 1 et 2) & \\
\hline Bisons d'Europe femelles & $79,5-79,5-82,2$ \\
\hline Bisons d'Amérique femelles & $80,4-82,0-82,3-82,3$ \\
\hline Bisons d'Europe mâles & $74,2-75,8-76,2$ \\
\hline Bisons d'Amérique mâles & $73,2-78,2$ \\
\hline Autres bisons fossiles d'Habarra & \\
\hline Bison femelle n' 1AB & 79,3 \\
\hline Bison femelle n' 4E & 80,2 \\
\hline Bison mâle n 2AB & 76,7 \\
\hline
\end{tabular}

\subsubsection{Le bison $n^{\circ} 8 \mathrm{D}$ mâle}

\subsubsection{Tibia gauche D91 (tableau 27, figure 20)}

Un peu moins grand et massif que les tibias du bison mâle $2 A B$, il ne se rapporte à aucun des bisons d'Habarra déjà cités dont les tibias n'ont pas été découverts. Ses dimensions dépassent très sensiblement celles des tibias des femelles $n^{\circ} 1 \mathrm{AB}, \mathrm{n}^{\circ} 4 \mathrm{E}$, 
$\mathrm{n}^{\circ} 5 \mathrm{E}$ et, a fortiori, devait dépasser celles des tibias (non trouvés) des bisons $\mathrm{n}^{\circ} 3 \mathrm{~B}, \mathrm{n}^{\circ} 6 \mathrm{~F}$ et $n^{\circ} 7 \mathrm{D}$ certainement plus petits que ces derniers. Nous l'attribuons donc à un mâle adulte.

Le condyle latéral de sa région proximale est franchement convexe vers le haut et les crêtes d'insertions musculaires de sa face postérieure sont dispersées comme chez les autres bisons d'Habarra (fig. 20-5). Elles sont remarquablement vigoureuses, plus saillantes même que chez le bison $n^{\circ} 2 \mathrm{AB}$ : elles indiquent un âge avancé.

6.5.2.2 Pièces du secteur $D$ paraissant se rapporter à un bison mâle moins grand et moins puissant que le sujet $n^{\circ} 2 A B$ donc, peut-être, au bison $n^{\circ} 8 \mathrm{D}$

6.5.2.2.1 Fragments proximaux d'une cheville osseuse de corne

Ces fragments, après reconstitution partielle de la cheville osseuse, permettent d'estimer à $100 \mathrm{~mm}$ environ le diamètre antéro-postérieur de la couronne.

\subsection{Ulna gauche D30-31 (tableau 16)}

L'ulna est pratiquement réduit à son olécrâne. Celui-ci est seulement un peu moins haut et moins large que celui des ulnas du bison $n^{\circ} 2 \mathrm{AB}$.

\subsection{Radio-ulnaire gauche D16 (tableau 15)}

Dépourvu de son olécrâne. il est plus allongé et plus robuste que ceux des bisons femelles d'Habarra. L'état de la face palmaire de son extrémité proximale, très friable, n'autorise pas une mise en connexion avec l'olécrâne D30-31. Peut-il être associé au tibia D91 ? Le rapport centésimal de sa longueur à la longueur de ce dernier égale 79,0.

Valeur de ce rapport chez d'autres bisons mâles :

Habarra $n^{\circ} 2 \mathrm{AB}: 79,4$

Bison bonasus : $76,58-76,70-76,85-77,24$

Bison bison : 81,68-85,14-85,31.

Les dimensions du radio-ulnaire D16 ne sont peut-être pas incompatibles avec celles du tibia D91. Nous préférons nous en tenir à cette seule conclusion.

\subsection{Unciforme gauche D87 (tableau 20)}

6.5.2.2.5 Partie proximale D87 d'un fémur gauche (tableau 26)

Cassé au-dessous du sommet de la fosse supra-condylaire.

\subsection{Calcanéum droit DB2 (tableau 33)}

Relativement grand et massif.

\subsection{Cubo-naviculaire droit D51 (tableau 30)}

Entre en contact, comme il faut, avec la pièce précédente mais, bien sûr, suivant une zone articulaire très étroite. 


\subsection{Vertèbre D54} C'est une 3ème cervicale plus grande et beaucoup plus robuste que la C3 du bison femelle $n^{\circ} 4 \mathrm{E}$.

\subsubsection{Le bison $n^{\circ}$ 9D femelle}

\subsubsection{Le métacarpe gauche D61 (tableau 22, figure 17-5)}

Il diffère des métacarpes des divers individus que nous avons présentés jusqu'ici. Plus allongé que le métacarpe du sujet femelle $1 \mathrm{AB}$, plus massif et plus court que celui de la grande femelle $4 \mathrm{E}$, il est moins trapu que les os canons antérieurs du mâle $\mathrm{n}^{\circ} 2 \mathrm{AB}$. Avec une certaine réserve nous l'attribuons à une femelle, nécessairement plus puissante que la bisonne $\mathrm{n}^{\circ} 6 \mathrm{~F}$, en soulignant que sa diaphyse est franchement rétrécie en son milieu et sa fossette synoviale ouverte vers l'arrière.

\subsubsection{Le métatarse gauche D72 (Bison $n^{\circ} 9 \mathrm{D}$ ?) (tableau 34)}

Nous aurions pu, tout aussi bien, fonder l'hypothèse de la présence d'un bison $\mathrm{n}^{\circ} 9$ dans le secteur D sur l'existence de cette pièce. Plus grand et moins massif que le métatarse $\mathrm{A} 13 \mathrm{du}$ bison $\mathrm{n}^{\circ} 1 \mathrm{AB}$, moins grand et relativement un peu plus large que les métatarses des autres femelles $n^{\circ} 4 \mathrm{E}$ et $n^{\circ} 5 \mathrm{E}$, il se révèle un peu moins long et plus « gracile » que ceux du mâle $\mathrm{n}^{\circ} 2 \mathrm{AB}$.

Le rapport centésimal de la longueur du métacarpe D61 en fonction de la longueur du métatarse D72, égal à 81,1 , ne diffère guère des rapports correspondants obtenus à partir de squelettes de bisons actuels ou fossiles, mâles et femelles (tabl. 1 et 2).

\subsubsection{Le bison $n^{\circ} 10 \mathrm{D}$ mâle}

\subsubsection{Le fémur droit D56-77 (tableau 26)}

Une tête fémorale D77, à l'état d'épiphyse isolée, se raccorde de façon adéquate à une portion proximale de fémur exépiphysée, D56. L'ensemble reconstitué indique un sujet de grande taille, de sexe mâle. Chez le Bison d'Europe la soudure des épiphyses du fémur intervient selon Koch (1934) vers le milieu de la 5ème année.

6.5.4.2 Autres pièces du secteur $D$ évoquant un sujet mâle immature (bison $n^{\circ} 10 D$ ?) étant donné leurs fortes dimensions, leur robustesse et leur degré d'ossification.

\subsection{Atlas D67}

Moitié latérale

\subsection{Axis D68}

Son épiphyse caudale, détachée, manque. 


\subsubsection{3 $5^{\text {ème }}$ vertèbre cervicale}

Vertèbre dépourvue de son épiphyse caudale et dont l'épiphyse crâniale n'est pas intimement soudée au corps

\subsubsection{4 $6^{\text {ème }}$ vertèbre cervicale D64}

Épiphyses apparemment rattachées au corps par des sédiments durcis. Peut-être faut-il associer à ces restes la scapula E55 déjà évoquée.

\subsubsection{Bison $n^{\circ}$ 8D ? Bison $n^{\circ} 10 D$ ?}

\subsubsection{Les métacarpes D78 gauche et droit (tableau 22)}

Ils tendent à se désagréger mais restent en partie mesurables. Ce sont les plus grands canons antérieurs trouvés à Habarra. En raison de leur taille, de leur caractère massif et de la forme de leur diaphyse à bords parallèles, nous sommes conduits à les rapporter à un bison mâle.

D'après Koch (1934), chez le Bison d'Europe, l'épiphyse des éminences articulaires des métacarpes se soude à la diaphyse vers la fin de la 4ème année. Les spécimens D78 d'Habarra, qui ne portent plus trace de cette fusion peuvent-ils, hypothétiquement, être associés au tibia D91 (sujet n $8 \mathrm{D}$ mâle) ? Ce n'est guère évident. En effet le rapport de leur longueur à celle de ce tibia atteint 52,3. Or, la valeur la plus élevée de ce rapport évaluée à partir des squelettes de bisons mâles (B. bonasus et B. bison) actuels, malheureusement peu nombreux, n'égale que 51,1. Chez le mâle $n^{\circ} 2 \mathrm{AB}$ d'Habarra le rapport est plus faible : 48,1 .

En conséquence si notre documentation, en raison de sa pauvreté, ne nous permet pas d'affirmer que les longueurs des métacarpes D78 sont incompatibles avec celles du tibia D91, elle ne nous incite sûrement pas à admettre le contraire.

D'autre part, la soudure des épiphyses des os canons ayant lieu plus tôt que celle des épiphyses du fémur, il n'est pas plus risqué de rapprocher les métacarpes D78 du fémur D56-77 (bison $n^{\circ} 10$ mâle) que du tibia D91 (bison n 8 mâle).

\subsubsection{Documents non attribués à un individu particulier}

\subsubsection{Les dents jugales supérieures D2 (tableau 11)}

\subsection{P4, M1, M2, M3 droites et P3, M1, M2 gauches}

On peut être tenté de rapprocher ces dents des pièces rapportées au bison $\mathrm{n}^{\circ} 7 \mathrm{D}$. En effet, en gisement, elles se trouvaient bien groupées auprès de l'humérus D1 et non loin du radio-ulnaire D7. Elles constituent, les premières une rangée droite incomplète, les secondes une partie de la série symétrique. D'autre part le degré d'usure de ces dents, si l'on se réfère aux figures publiées par Skinner et Kaisen (1947, pl. 9, fig. 7), indique un sujet adulte jeune : l'abrasion a nettement, mais modérément, entamé les cônes de M3 sans atteindre l'entostyle, parfaitement intact, comme celui de M2. Cette colonnette interlobaire, très courte, fusiforme, s'efface dans la région cervicale de la couronne, conformément à ce que l'un de nous considère comme une des caractéristiques de la denture supérieure des bisons (Delpech 1984). 
312 Sur M1 les cuspides sont saillantes par rapport au sillon vestibulo-lingual médian de la face occlusale, moins qu'elles ne le sont sur M2 et M3. En ce qui concerne l'hypothèse d'une possible appartenance au bison $\mathrm{n}^{\circ} 7 \mathrm{D}$, non à exclure, elle inspire beaucoup de retenue quand on sait qu'au même endroit gisait la molaire supérieure dont il est question ci-après.

\subsection{M2 gauche}

Elle est en tous points comparable à la M2 gauche de la série précédente (degré d'usure, dimensions, forme de l'entostyle...). Tout aussi bien que les dents décrites plus haut elle pourrait donc être jointe aux ossements du sujet $n^{\circ} 7 \mathrm{D}$. En fait, pas plus que les molaires déjà décrites elle ne peut être attribuée avec certitude à l'un des bisons déterminés du secteur $\mathrm{D}$.

\subsubsection{Fragment d'os temporal D11}

\subsubsection{Fragment d'os frontal D12}

\subsubsection{Fragments crâniens divers D15}

Ils ont appartenu à un (ou plusieurs) individu(s) non adulte(s). On distingue en particulier :

- une pièce comprenant une partie de pariétal et d'occipital avec protubérance nucale (ligne de suture pariéto-occipitale oblitérée)

- un fragment de maxillaire supérieur gauche réduit à la région alvéolaire des prémolaires

- un fragment de maxillaire supérieur gauche rongé

- une P3 supérieure gauche D15 (tabl. 11)

- une $\mathrm{P} 4$ inférieure gauche très usée dont le diamètre mésio-distal maximum occlusal égale 22 $\mathrm{mm}$ et le diamètre vestibulo-lingual $14,5 \mathrm{~mm}$.

\subsubsection{Molaire supérieure droite D27}

Hauteur de la couronne à l'extrémité apicale des cuspides vestibulaires: $23 \mathrm{~mm}$ environ. Diamètres mésio-distal et vestibulo-lingual au niveau occlusal : respectivement 30 et $24 \mathrm{~mm}$ environ.

\subsubsection{Os nasal droit isolé D52}

Détaché sans dommage d'un calvarium non retrouvé ou non identifié

Longueur totale : $199,5 \mathrm{~mm}$ $\mathrm{n}^{\circ} 7 \mathrm{D} ?$ ?).

\subsubsection{Vertèbre cervicale (C6) exépiphysée D9}

Dimensions relativement faibles 
6.5.6.8 Vertèbre cervicale (C4 ?) exépiphysée D34

\subsubsection{Vertèbre cervicale (C4) D49}

Indique un sujet mâle adulte plus puissant que le bison mâle $\mathrm{n}^{\circ} 8 \mathrm{D}$

\subsubsection{Vertèbre cervicale (C3 ?) D25}

Peut-être même individu que le précédent

Les vertèbres D49 et D25 représentent probablement un grand mâle distinct du bison $\mathrm{n}^{\circ} 2 \mathrm{AB}$ et du bison $\mathrm{n}^{\circ} 14$ (présenté plus bas).

\subsubsection{Fragments crâniens divers D55}

\subsubsection{Humérus gauche D80}

Sa région proximale est détruite et sa région distale très endommagée.

\subsection{Le secteur $\mathrm{H}$, gisement amont (fouilles et relevés $\mathrm{G}$. Marsan et $\mathrm{F}$. Certain)}

\subsubsection{Bison $\mathrm{n}^{\circ} 11 \mathrm{H}$ femelle}

\subsubsection{Métacarpe H1 droit (tableau 22, figure 17-3 et 8)}

Le secteur n'a livré jusqu'ici que quelques os de Renne et un os canon antérieur (H1) de Bison, le plus petit trouvé à Habarra. Il a été recueilli à environ $2,50 \mathrm{~m}$ des restes du bison $\mathrm{n}^{\circ} 7 \mathrm{D}$. Appartenait-il à cet individu ? Si l'on considère le rapport centésimal de sa longueur à celle du radius D7, cela paraît plausible : il égale 63,97 ce qui le situe dans l'éventail de variation (?) de cet indice chez le bisons femelles (cf. tabl. 1) :

\begin{tabular}{|l|l|}
\hline & Longueur métacarpe / Longueur radius * 100 \\
\hline Longueur Hl/Longueur D7 & 64,0 \\
\hline B. bison $\$(n=4)$ & $64,9-67,2-67,2-67,3$ \\
\hline B. bonasus $9(n=4)$ & $62,8-64,5-68,4$ \\
\hline B. prisais ${ }^{\circ}{ }^{d}$ 'Habarra & 66,0 \\
\hline$n^{\circ} 1 \mathrm{AB}$ & \\
\hline$n^{\circ} 4 \mathrm{E}$ & 65,2 \\
\hline
\end{tabular}

En revanche deux observations ne plaident pas en faveur de cette hypothèse :

1) L'indice de robustesse du métacarpe $\mathrm{H} 1$ indique un sujet plus trapu que la femelle $4 \mathrm{E}$ $\mathrm{d}^{\prime}$ Habarra alors que le radius $\mathrm{D} 7$ (bison $\mathrm{n}^{\circ} 7$ ) dénote au contraire un sujet plus gracile ( cf. tabl. 15 et 22) ; 
2) La longueur du métacarpe $\mathrm{H} 1$ semble ne pas être compatible avec celle des métatarses D59 et DB3 qui serait ( ?) en harmonie avec celle du radius D7.

\begin{tabular}{|c|c|}
\hline & $\begin{array}{l}\text { Longueur métacarpe / Longueur métatarse }{ }^{*} \mathbf{1 0 0} \\
\text { (cf. tabl. 1, 22et 34) }\end{array}$ \\
\hline Longueur H1/longueur D59 & 78,7 \\
\hline B. bison 우 $(\mathrm{n}=4)$ & $80,8-81,6-81,7-82,1$ \\
\hline B. bonasus $(\mathrm{n}=3)$ & $79,1-81,2-83,2$ \\
\hline B. bison ㅇ $(n=164)(*)$ & valeur moyenne : 80,1 \\
\hline B. priscus 우 d'Habarra & \\
\hline$n^{\circ} 1 \mathrm{AB}$ & 83,3 \\
\hline$n^{\circ} 4 \mathrm{E}$ & 81,4 \\
\hline
\end{tabular}

(*) d'après chiffres donnés par McDonald (1987, tabl. 30). En conséquence, en attendant la mise au jour de carpiens unissant parfaitement le radius $D 7$ au métacarpe $H 7$, nous rapportons ce dernier à un bison $n^{\circ} 11 \mathrm{H}$.

\subsection{Secteur J, gisement aval}

Sous-secteurs Ja et Jc (Fouilles, relevés et déterminations in situ J.-P. Lhomme, J.-F. Tournepiche, A. Turq)

Sous-secteur Jd (Fouilles, relevés et déterminations in situ G. Marsan)

Selon les observations des fouilleurs consignées dans le rapport rédigé par G. Marsan, le secteur $\mathrm{J}$ correspondrait à une véritable fosse à ossements. Il a été subdivisé en 4 soussecteurs $a, b, c$ et $d$, ayant chacun fait l'objet d'un plan de situation des pièces exhumées.

Nous examinerons en premier lieu le cas d'un calvarium provenant du sous-secteur Jd (Jd1), certes incomplet, mais moins détérioré que la portion crânienne découverte dans le sous-secteur Ja.

\subsubsection{Bison $\mathrm{n}^{\circ}$ 12Jd mâle(?)}

\subsubsection{Le crâne Jd1-Jd4 (tableaux 3, 4, 5 et $11 ;$ pl. 1 et 2)}

Le sous-secteur Jd a livré la « moitié » postérieure, Jd1, d'un crâne de bison non adulte auquel il faut sans doute associer un maxillaire supérieur gauche Jd4.

L'os frontal droit serait assez bien conservé mais il ne reste guère de son processus cornual que le col, la région de la couronne, trop endommagée pour permettre des mesures précises, et une partie de sa courbure dorsale. De l'orbite correspondante subsiste un petit segment dorso-caudal peu proéminent. 

souffert. décrits. anatomique.

L'os frontal gauche étant détérioré superficiellement dans sa partie latérale, les sinus sous-jacents se trouvent ainsi mis au jour. Du même côté le pariétal a également

Toute la face nucale, la partie basilaire de l'occipital, le corps du basisphénoïde et, dans une moindre mesure, la zone articulaire des temporaux, les bulles tympaniques et les processus mastoïdes sont conservés.

La croissance des différentes pièces crâniennes n'était pas terminée comme en témoigne le contact imparfait de la plupart des os adjacents. C'est notamment le cas de l'écaille de l'occipital et des deux exoccipitaux. Le corps du basioccipital et celui du basisphénoïde n'avaient pas davantage réalisé intimement leur jonction au niveau du tubercule musculaire. Celui-ci présente deux reliefs particulièrement saillants encore séparés l'un de l'autre. L'un, en position caudale, se développe à partir du basioccipital, l'autre, en position rostrale, moins proéminent, dépend du basisphénoïde. Une crête régulière, relativement épaisse, les relie incomplètement l'un à l'autre.

Faiblement convexe à partir de la protubérance nucale, le profil dorsal décrit une légère ensellure sus-orbitaire avant d'ébaucher une convexité peu accusée, interrompue par la ligne de fracture et de désarticulation majeure qui passe à peu près par l'épine nasale des os frontaux.

7 Le processus cornual droit réalise avec le plan sagittal un angle caudal d'environ $70^{\circ}(\mathrm{pl}$. 1), plus grand donc que celui mesuré sur les crânes des deux femelles E12 (bison $\mathrm{n}^{\circ} 4 \mathrm{E}$ ) et $\mathrm{F} 10$ (bison $\mathrm{n}^{\circ} \mathrm{6F}$ ). Les points les plus ventraux de sa courbure dorsale, s'il est permis d'en juger malgré le mauvais état de conservation de celle-ci, se situent au niveau du plan des os frontaux (pl. 2). Nous retiendrons surtout la valeur du diamètre dorsoventral de son col $(89 \mathrm{~mm})$ nettement plus forte que celle relevée sur les crânes des deux femelles citées (tabl. 5). Au-delà du col, la surface non détériorée (peu étendue) de la cheville osseuse est poreuse et lisse : on n'y voit aucune trace des cannelures et reliefs longitudinaux présents chez les adultes.

Le sillon supra-orbitaire droit est profond par rapport à ce qu'il est sur les crânes déjà

En vue latérale on constate que les condyles de l'occipital font saillie en arrière de l'écaille et de la protubérance nucale.

En vue postérieure, en raison de la netteté de la limite temporo-occipitale, de chaque côté, on constate que la crête nucale et la crête mastoïdienne, dans le prolongement l'une de l'autre, demeurent bien distinctes. Elles dessinent une courbe régulière et s'épaississent à leur point de rencontre.

1 En vue basale les deux reliefs caudaux du basioccipital sur lesquels s'appuient respectivement les revers ventraux des condyles, relativement larges et épais, divergent vers l'arrière, surtout si l'on considère leur bord latéral.

\subsubsection{Le maxillaire supérieur Jd4 (tableau 11)}

42 En gisement il se trouvait juste au-dessous de la cheville osseuse droite du calvarium mais les dégâts subis par les deux pièces rendent vains les essais de remise en place

3 Cette portion de mâchoire est essentiellement réduite à la région d'implantation des dents jugales parmi lesquelles P4, M1, M2 et M3 demeurent en place. Le tubercule facial 
est modérément épais et proéminent. Sa partie la plus saillante se situe à l'aplomb du lobe mésial de M1. Au-dessous il se prolonge par une crête discrète jusqu'au niveau du bord mésial de P4. Au-dessus, la crête faciale, également peu accentuée, oblique dans le sens rostro-caudal s'efface à l'aplomb du lobe mésial de M1.

Le crâne d'un jeune bison mâle d'Amérique conservé à l'Institut de Préhistoire et de Géologie du Quaternaire de l'Université Bordeaux I constitue ici un bon terme de comparaison. Aucun de ses os n'est en contact très étroit avec les pièces voisines. La surface de ses chevilles osseuses de corne est poreuse et lisse et ses orbites sont relativement peu saillantes. Ses dents lactéales D3 (à lobe mésial très usé) et D4 étaient encore en activité ; la P2, en cours d'éruption, n'avait pas atteint le niveau occlusal, pas plus que la M3 encore bien enfoncée dans son alvéole. Cette disposition correspond assez bien au stade 5 défini par Skinner et Kaisen (loc. cit. p. 145, pl. 8-5c) qui caractériserait la première adolescence. D'après Fuller (1959, p. 344), c'est au cours de la 3ème année que $\mathrm{P} 2$ et $\mathrm{P} 3$ remplacent respectivement $\mathrm{D} 2$ et $\mathrm{D} 3$ et que M3 apparaît chez B. bison. D4 perdure souvent plus longtemps que les deux autres molaires lactéales mais il lui arrive aussi de chuter avant celles-ci. Etant donné le stade d'usure de D3 on peut penser que l'animal en cause était dans sa troisième année.

Le calvarium Jd1 diffère du précédent par ses dimensions plus fortes et la soudure mieux réalisée de ses os frontaux qui n'auraient sans doute pu se disjoindre sans quelques dommages. Le stade d'évolution dentaire des molaires du maxillaire Jd4 fournit quelques informations complémentaires. L'usure avait entamé les cônes de P4 ainsi que le métacône et l'hypocône de M3. Si l'entostyle de M2 et M3 est encore intact, celui de M1 est franchement abrasé. Ce sujet avait donc certainement atteint, et peutêtre dépassé, l'âge de 3 ans. Si l'on s'en remet à Skinner et Kaisen le degré d'évolution dentaire que nous observons correspondrait à l'adolescence tardive. Il va de soi que ces conclusions ne seront admises qu'avec réserve puisqu'elles s'appuient sur des observations effectuées chez l'espèce actuelle d'Amérique du Nord.

Nous nous montrerons également réservés quant à la détermination du sexe. Certaines dimensions crâniennes s'écartent peu de celles relevées sur le crâne des femelles E12 et F10 alors que d'autres sont sensiblement plus grandes.

D'après Skinner et Kaisen (loc. cit. p. 147) la longueur du crâne d'un bison parvenu au stade de l'adolescence ne mesure que les 4/5ème de sa longueur définitive. Certes le crâne du bison $n^{\circ} 12 \mathrm{Jd}$ en était (?) au stade de l'adolescence tardive mais on peut imaginer que si l'animal avait vécu un peu plus longtemps, la largeur du frontal mesurée entre la suture sagittale et le point le plus médial de la couronne du processus cornual, qui égale $137 \mathrm{~mm}$ environ, eut été alors plus proche de celle du crâne du mâle $\mathrm{n}^{\circ} 2 \mathrm{AB}(165 \mathrm{~mm})$ que de celle du crânes des femelles $\mathrm{n}^{\circ} 4 \mathrm{E}$ et $\mathrm{n}^{\circ} 6 \mathrm{~F}$ (respectivement 112 et $113 \mathrm{~mm}$ ). Nous serions ainsi plutôt enclins à considérer que les vestiges crâniens du sous-secteur Jd sont ceux d'un individu de sexe mâle.

La découverte dans la même zone d'une vertèbre dorsale a bien été signalée mais la pièce n'a pas été retrouvée.

\subsubsection{Bison $n^{\circ}$ 13Ja mâle ( ?)}

Cette partie du secteur J a surtout livré quelques côtes et vertèbres, de nombreuses esquilles, un tibia (Ja29) non retrouvé et une portion de crâne. 


\subsubsection{Le crâne Ja19 (tableau 3)}

350 A peu près réduit à son frontal gauche, il conserve la base de sa cheville osseuse de corne ainsi qu'une partie de l'orbite. En examinant le bord médial de la pièce, on voit bien que l'union des os frontaux était vraisemblablement moins intime que sur le spécimen Jd1 (bison $\mathrm{n}^{\circ} 12 \mathrm{Jd}$ ) : les deux os se sont séparés l'un de l'autre sans opposition.

Entre la suture sagittale et le bord dorsal de la couronne de la cheville osseuse (plus exactement de son point le plus médial) la largeur du frontal mesure environ $137 \mathrm{~mm}$, comme chez le bison $n^{\circ} 12 \mathrm{Jd}$ qui paraît un peu plus âgé.

Dans l'hypothèse où nous aurions affaire à un individu n'ayant pas atteint le stade de l'adolescence tardive, on peut supposer que la taille du crâne de cet individu parvenu à l'âge adulte eut pu dépasser sensiblement celle du crâne des femelles $\mathrm{n}^{\circ} 4 \mathrm{E}$ et $\mathrm{n}^{\circ} 6 \mathrm{~F}$. Nous pourrions donc avoir affaire, comme dans le cas du crâne 12Jd à un jeune « taurillon».

\subsubsection{Bison $n^{\circ} 14 \mathrm{Jc}$ mâle}

\subsubsection{Le crâne Jc63 (tableaux 3 et 5 ; pl. 1 et 2)}

Au moment de sa découverte, le crâne Jc63, figuré à l'échelle de 1/10ème sur le plan, était orné de ses processus cornuaux complets dont l'envergure atteignait $1200 \mathrm{~mm}$ environ, soit l'ordre de grandeur de celle des processus cornuaux du crâne de Lurbe (tabl. 5). Malheureusement, en raison de l'extrême fragilité de leur partie distale, ces chevilles osseuses n'ont pu être intégralement conservées.

Il ne reste rien du massif facial. En arrière des arcades sourcilières, la région frontale est pratiquement seule étudiable. Le pariétal, détérioré caudalement, ne fait qu'amorcer la saillie nucale dont on ne peut évaluer le développement.

En vue nucale, les os frontaux décrivent chacun une convexité prononcée qui correspond à la face dorsale du col du processus cornual. Vus de face, de part et d'autre de la ligne de suture le long de laquelle ils forment un léger bourrelet, ils apparaissent presque plats dans leur ensemble, voire légèrement concaves, avant de décrire la convexité précédemment signalée.

Entre les deux chevilles osseuses la largeur frontale, mesurée au bord dorsal des deux couronnes, est inférieure à celle du crâne A55-E1 (bison $n^{\circ} 2$ ) mais à peu près égale à celle du crâne de Lurbe (tabl. 3).

L'occipital et les temporaux étant presque totalement détruits, il n'y a rien à voir en vue caudale et en vue ventrale sauf la paroi interne de la voûte dorsale protégeant la cavité cérébrale.

Le processus cornual droit s'est brisé à environ $363 \mathrm{~mm}$ du bord dorsal de la couronne (mesure prise en ligne droite) et la face ventro-caudale de la partie restante a ellemême subi de gros dommages. Son symétrique n'a pas moins souffert : il ne mesure que $325 \mathrm{~mm}$. Les deux appendices, détériorés, corrodés superficiellement, sont creusés de sinus jusqu'au niveau de la cassure.

Comme sur le crâne du bison mâle $n^{\circ} 2 \mathrm{AB}$, chaque processus cornual, dans son mouvement de latéralité, se dirige d'abord nettement vers le bas et l'arrière, son axe réalisant avec le plan sagittal un angle caudal de $76^{\circ}$, plus grand que l'angle correspondant mesuré sur les crânes E12 et F10 attribuables à des femelles 
(respectivement $59^{\circ}$ et $63^{\circ}$ ). Ces appendices, dont nous ignorons s'ils dépassaient ou non le plan de l'os occipital, se dressent ensuite en décrivant une courbe moins accusée que celle des chevilles osseuses du bison $\mathrm{n}^{\circ} 2 \mathrm{AB}$ aux cornes moins étalées (pl. 2). Cependant, comme chez ce dernier et contrairement à ce que nous constatons sur les spécimens E12 et F10, les points les plus ventraux de l'arc supérieur se situent audessous du plan des os frontaux. En regardant les processus cornuaux (ou plutôt ce qu'il en reste), perpendiculairement à leur face latérale, nous ne décelons pas l'ébauche d'un changement de direction de ces appendices qui tendrait à diriger vers l'avant ou vers l'arrière leur partie distale.

Il faut souligner enfin qu'au niveau de la couronne et du col le périmètre et les diamètres rostro-caudal et dorso-ventral des axes osseux des cornes sont plus forts que chez le bison $n^{\circ} 2 \mathrm{AB}$.

Nous rapportons, bien entendu, le calvarium Jc63 à un mâle adulte. Son attribution au bison $\mathrm{n}^{\circ} 8 \mathrm{D}$, de même sexe mais de stature médiocre, ne paraît pas s'imposer.

\subsubsection{Bison $\mathrm{n}^{\circ} 15 \mathrm{Jc}$ femelle}

Quelques ossements de Bison découverts à proximité du crâne $n^{\circ} 63$ n'appartiennent pas au sujet mâle $\mathrm{n}^{\circ} 14 \mathrm{Jc}$.

\subsubsection{L'humérus gauche Jc61 (tableau 14)}

Sa longueur égale, à très peu près, celle de l'humérus E39 de la femelle $\mathrm{n}^{\circ} 4$ mais sa robustesse est moindre. D'autre part la ligne de suture de son épiphyse proximale reste nette sur tout son pourtour, ce qui indique un sujet relativement jeune.

Proviennent, selon toute vraisemblance, du même squelette :

\subsubsection{Le fémur droit Jc45 (tableau. 26)}

Il est endommagé tant à son extrémité supérieure qu'à son extrémité inférieure

\subsubsection{Le fémur gauche Jc43 (tableau 26)}

C'est le symétrique du précédent. Sa région proximale a subi de très gros dégâts mais sa région distale, moins détériorée, présente une ligne de suture épiphysaire oblitérée par endroits. A rappeler que chez le Bison d'Europe, d'après Koch (1934), en ce qui concerne l'humérus, les soudures des épiphyses proximales se font vers 6 ans alors que la soudure de l'épiphyse distale du fémur se réalise au cours de la 2ème moitié de la 5ème année.

\subsubsection{Le talus Jc62}

Il est presque réduit à sa trochlée proximale. 


\subsubsection{Bison $\mathrm{n}^{\circ} 16 \mathrm{Jc}$ femelle}

\subsubsection{L'humérus droit Jc62}

Il n'en reste qu'un fragment de diaphyse qui correspond à un os moins large que l'humérus Jc61.

\subsection{Secteur M, gisement aval (Fouilles, relevés et déterminations in situ E. de Valicourt et F.M. Callot)}

\subsubsection{Bison $\mathrm{n}^{\circ} 17 \mathrm{M}$ mâle}

Ce secteur comprend une zone haute et une zone basse. De la zone haute ont été exhumés quelques os fragmentaires ainsi qu'un radius et un ulna M4 dépourvus d'épiphyse distale. Il faut souligner que leurs dimensions (tabl. 15) dépassent, pour la plupart, celles des os correspondants du bison $n^{\circ} 2 \mathrm{AB}$. En conséquence nous les rapportons à un mâle de haute stature n'ayant pas atteint 6 ans.

On se souvient que dans le secteur $\mathrm{D}$ du gisement amont se trouvaient les restes (vertèbres et fémur) d'un grand mâle immature $\mathrm{n}^{\circ}$ 10D. En l'état des découvertes il serait téméraire de lui rapporter le radio-ulnaire du secteur $\mathrm{M}$.

Le secteur $M$ recèle toujours ( ?) un crâne de bison très friable qui n'a pu être dégagé de sa gangue de calcite.

La zone basse, quant à elle, a livré une portion d'omoplate droite M11 dont nous ignorons si elle appartient ou non au bison $n^{\circ} 17 \mathrm{M}$ (tabl. 13).

\subsection{Secteur?}

\subsubsection{Bison $n^{\circ} 18$ mâle}

Nous ne savons de quel secteur de la caverne provient un radio-ulnaire gauche (tabl. 15) marqué 1 . Ses épiphyses distales, détachées, n'ont pas été retrouvées. Cet os appartenait à un sujet mâle non adulte aussi grand que le bison $n^{\circ} 17 \mathrm{M}$.

\section{Conclusions}

Au total 18 bisons en provenance du gisement d'Habarra ont été distingués à ce jour, 10 femelles et 8 mâles, adultes ou non (cf. tableau 42 indiquant la répartition des vestiges par individu). Il ne s'agit là que d'une approximation, plusieurs pièces n'ayant pu être rapportées à l'un ou à l'autre des individus identifiés alors que d'autres ne l'ont été qu'avec réserve. Dans certains cas nos décisions ont pu conduire à une surestimation de l'effectif. Est-il bien sûr, par exemple, que la mandibule B88 qui, dans le secteur B représenterait seule le bison $n^{\circ} 3$, n'appartenait pas au bison $n^{\circ} 5$ du secteur $E$ dont nous ne possédons que des os des membres postérieurs? En fait il est probable que le nombre indiqué plus haut ne donne qu'une faible idée de la représentation de l'espèce dans la grotte puisque la couche ossifère n'a été qu'à peine entamée. 
On notera aussi, sans en tirer de conclusions en l'état actuel des découvertes, que les femelles semblent majoritaires dans le gisement du haut (8 sur 10) et les mâles dans celui du bas ( 5 sur 8 ).

Nous avons assez peu insisté sur les dispositions anatomiques qui, sur les os de Bovinés, désignaient le Bison et non le Bœuf. La morphologie des crânes et des os des membres, souvent bien conservés, ne laissait guère place au doute. Cependant nous avons une fois encore constaté que les caractères déterminants, très justement mis en évidence par divers auteurs, n'apparaissent pas toujours avec la même netteté et la même constance. Il en a été donné quelques exemples au passage. Il faut donc comprendre l'embarras des paléontologistes qui, sachant que Bison des steppes et Aurochs ont parfois cohabité, doivent fréquemment établir leur diagnose sur des os fragmentaires ce qui les engage à partir à la recherche de nouveaux critères d'identification générique. Ce ne fut donc pas notre cas : dès le début l'appartenance des ossements de Bovinés d'Habarra à Bison priscus apparut évidente.

Les précisions chronologiques étant momentanément insuffisantes nous ne pouvons entrevoir l'ordre de mise en place des carcasses et tenter une détermination des événements qui ont amené leur démantèlement et leur dispersion. D'autre part, l'examen des plans qui montrent de quelle façon se situent, les uns par rapport aux autres, les éléments des squelettes gisant dans un même secteur, n'apporte guère d'information à ce sujet. Considérant les relevés des plans $\mathrm{A}$ et $\mathrm{B}$ nous constatons ce qui suit :

- les os des bisons $\mathrm{n}^{\circ} 1 \mathrm{AB}$ et $\mathrm{n}^{\circ} 2 \mathrm{AB}$ ne se chevauchent pas ;

- la branche montante de la mandibule A59 de Rhinocéros recouvre en partie le métatarse A47 du bison $n^{\circ} 2 \mathrm{AB}$ tandis que son apophyse angulaire se place sur le calcanéum A46 et le talus A67 du même bison

- les premières phalanges A53, A54 et $\mathrm{A} 60$ du bison $n^{\circ} 2 \mathrm{AB}$ reposent sur la scapula $\mathrm{A} 61 \mathrm{du}$ rhinocéros;

- une partie du corps de la vertèbre thoracique $A 89$ du bison $n^{\circ} 2 A B$ est sous-jacente à l'acétabulum du coxal A86 de Rhinocéros alors que l'extrémité distale de son processus épineux recouvre le bord de l'ilium de ce coxal.

Il est pourtant vrai que dans les secteurs $\mathrm{D}$ et $\mathrm{E}$, chaque fois que les restes de rhinocéros et d'un bison étaient découverts en superposition, l'os du rhinocéros se trouvait audessus de celui du bison. Cela ne signifie pas nécessairement que le bovidé habitait la région dans des temps plus anciens mais, peut-être, qu'il est tombé dans le gouffre un peu plus tôt mettant ainsi un terme à la cohabitation des deux bêtes. On ne peut davantage exclure l'hypothèse selon laquelle la répartition actuelle des restes serait le résultat de déplacements postérieurs à la chute des animaux.

En ce qui concerne les bisons femelles $4 \mathrm{E}$ et $5 \mathrm{E}$ on remarque sur le plan (fig. 24) que le fémur E2 du sujet $\mathrm{n}^{\circ} 4 \mathrm{E}$ surmontait le métatarse $\mathrm{E} 16$ du bison $\mathrm{n}^{\circ} 5 \mathrm{E}$ alors que la vertèbre thoracique E73 de celui-ci reposait en grande partie sur la mandibule et le métatarse de la vieille bisonne.

Les bisons d'Habarra, comme les individus des autres espèces présents dans le site, devraient être datés individuellement. A ce jour on n'a que peu d'informations à ce sujet mais les trois datations concernant les chevaux du secteur $\mathrm{K}$, le Cerf du secteur $\mathrm{L}$ et un bison du secteur $\mathrm{E}$ indiquent que la grotte d'Habarra a constitué un piège naturel pour la grande faune pléistocène au cours du Würm récent, au moins de 28-29000 à 
15-14000 ans BP. Des grands bovidés d'Habarra, seule la bisonne $4 \mathrm{E}$ a été datée : son radius gauche $\mathrm{E} 45$, symétrique de $\mathrm{E} 47$, indique un âge ${ }^{14} \mathrm{C}$ de $14500 \pm 260 \mathrm{BP}$ (Gif 7106). Nous croyons donc que Bison, Renne et Rhinocéros laineux, dont les os se mêlent plus ou moins au sommet du remplissage de la caverne d'Habarra, vivaient au même moment dans la région d'Arudy, pendant une période froide et relativement sèche.

Encore assez peu favorable aux grands Ongulés, cette période ( vers $14500 \mathrm{BP}$ ) est nettement moins contraignante que les précédentes, proches et contemporaines du dernier maximum glaciaire que l'on situe vers 18500 BP. Les espaces d'altitude, qui constituaient jusque là des déserts froids, intègrent de nouveau la biosphère (Delpech, 1999a, 1999b, 2003; Delpech et Lenoir, 1996). La bisonne $4 \mathrm{E}$ et ses congénères contemporains font certainement partie des premiers ongulés recolonisateurs des montagnes Pyrénées restées désertiques pendant plusieurs milliers d'années. Sachant que le piège d'Habarra a commencé à fonctionner au moins vers 28-29000 BP, certains bisons représentés dans la caverne ont pu tomber, comme des chevaux du secteur $\mathrm{K}$ et des cerfs du secteur L, lors de la première moitié du Würm récent avant que le désert froid du maximum glaciaire ne se soit mis en place. De nouvelles datations sont nécessaires pour aller plus loin dans l'avancée des hypothèses de chronologie et nous considérons les restes de Bison d'Habarra comme représentant une population de Bison priscus du Würm récent pyrénéen.

Tout porte à croire que la taille des bisons d'Habarra n'atteignait pas (du moins en moyenne) celle des bisons plus anciens (ante-würmiens) de Mosbach (Rhénanie), de Châtillon-Saint-Jean (Drôme) et de Riverenert (Ariège). La lecture des tableaux 22 et 34 est à ce sujet très instructive.

Le nombre, relativement élevé de talus de Bison recueillis par F. Daleau à Pair-non-Pair en Gironde (48 conservés au Muséum d'Histoire Naturelle de Bordeaux) nous a suggéré l'idée de tracer un diagramme de dispersion de ces pièces en tenant compte de leur longueur et de leur largeur et de faire figurer parmi eux (ou à leur côté) ceux d'Habarra (fig. 27). Certes la situation dans le temps du gisement girondin demeure relativement imprécise et l'on peut encore reprendre à leur sujet ce que l'un de nous (F.P.) écrivait en 1968 (p. 329) : « ils ne sont... vraisemblablement ni antérieurs au Würm » ancien « ni de beaucoup postérieurs (s'ils le sont) au début du Würm »récent. Il est même probable que la plupart des restes de bisons, chevaux, mammouths et rhinocéros (Coelodonta antiquitatis), souvent peu brisés quand il s'agit d'os particulièrement compacts (os canons des Equidés et Bovinés, astragales et calcanéums en tout genre) proviennent d'une couche à ossements résultant d'une occupation de la grotte de Pair-non-Pair par l'Hyène des cavernes (Crocuta spelaea) elle-même très présente. Cela plaiderait en faveur de l'hypothèse d'une certaine homogénéité chronologique des talus mesurés. 
Figure 27 - Bison priscus. Diagramme de dispersion des talus de Pair-non-Pair (Gironde) et d'Habarra (Pyrénées Atlantiques).

Figure 27 - Bison priscus. Scattergram of talus measurements for specimens from Pair-non-Pair (Gironde) and Habarra (Pyrénées Atlantiques).

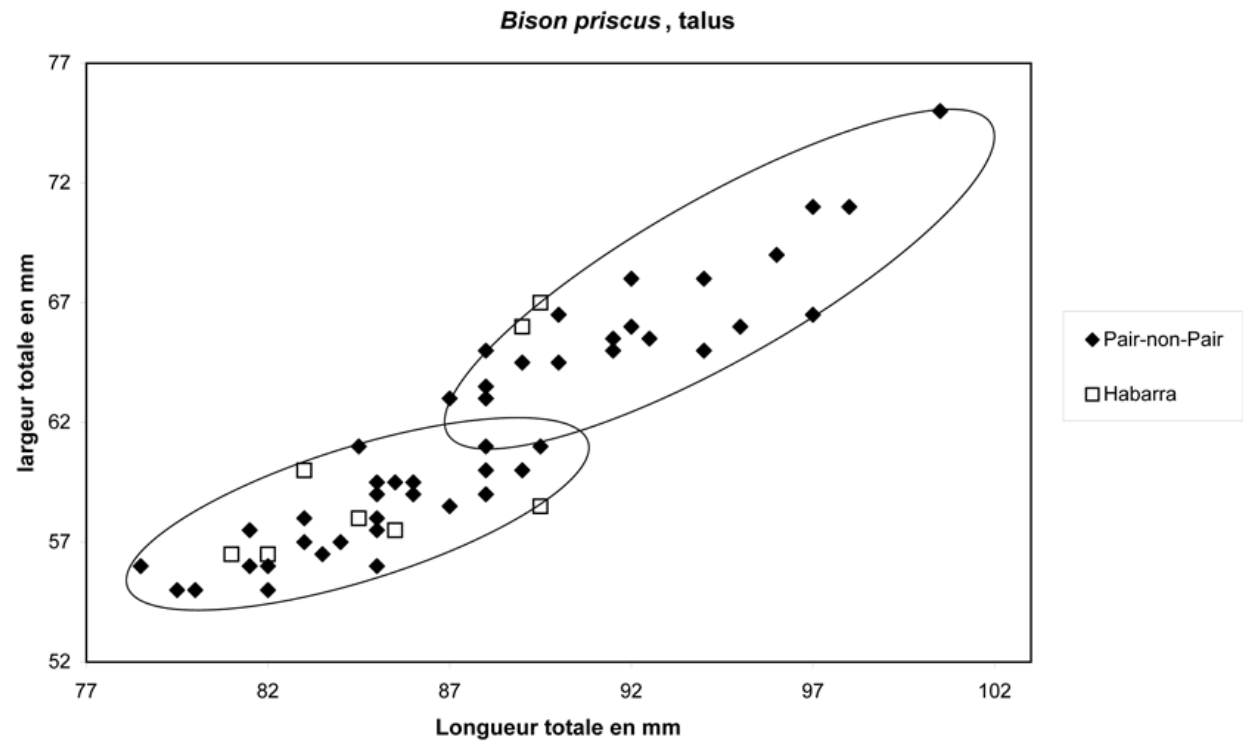

Deux ensembles de dimensions comparables tendent à s'individualiser. L'un, un peu moins dense, comprend des talus souvent plus grands et presque toujours plus massifs : il correspondrait, grosso modo, à des sujets mâles. Le bison $n^{\circ} 2 \mathrm{AB}$ d'Habarra y figure, à peu près en position moyenne, mais on remarque que ses astragales se révèlent relativement trapus. Dans l'autre ensemble, conformément à ce que l'on pouvait attendre, se trouvent les femelles $1 \mathrm{AB}, 5 \mathrm{E}, 7 \mathrm{D}$ et, bien entendu, $4 \mathrm{E}$ qui verrait son statut de femelle exceptionnellement "élancée» confirmé, du moins dans le cadre chronologique (würmien) et géographique (Aquitaine) considéré. Selon toute probabilité le Bison d'Habarra ne différait guère de celui de Pair-non-Pair. Reste à savoir si des découvertes plus substantielles ne viendront pas infirmer cette opinion. En effet, il est possible qu'au cours du temps, même à l'échelle d'une période glaciaire, et suivant les lieux, la stature et la robustesse des individus au sein des troupeaux de bisons aient varié en fonction de l'abondance et/ou de la qualité de la nourriture. Aujourd'hui il semble difficile de déterminer ce qui distingue le Bison d'Habarra de ses congénères pléistocènes, soit un peu plus anciens, soit contemporains ou plus récents. Quoi qu'il en soit, que des bisons datés de 28-29000 à 14500 BP apparaissent assez proches de ceux de Pair-non-Pair n'a rien de très surprenant.

Ce que l'on retiendra aussi du diagramme de la figure 27 c'est l'existence de deux ensembles qui témoignent d'un net dimorphisme sexuel. Ceci nous conduit à souligner l'importance de l'identification du sexe (pas toujours évidente, peut-être impossible parfois) dans toute étude comparative relative à l'espèce. Avant de revenir sur ce problème à propos du Bison des steppes, nous indiquerons brièvement en quoi cette espèce se distingue de ses congénères actuels.

Poplin (1984) a étudié le profil de la ligne du dos chez les Bisons d'Amérique, d'Europe et Bison priscus prenant en compte, pour ce dernier, non seulement la forme du rachis mais aussi les représentations paléolithiques. Il mit ainsi en évidence des différences dans le modelé et la position de la bosse entre les trois espèces, différences liées au plus ou moins grand développement du processus épineux des vertèbres thoraciques. Nous 
nous bornerons à rappeler ici que chez B. bison la ligne dorsale déterminée par le sommet des dits processus s'abaisse de façon régulière du sommet de la bosse à la croupe tandis que chez B. bonasus et B. priscus la partie postérieure de la bosse, qui décrit une nette concavité, se trouve bien dégagée vers l'arrière. Malheureusement aucun des squelettes d'Habarra, y compris les moins incomplets ( $n^{\circ} 1 \mathrm{AB}, 2 \mathrm{AB}$ et $\left.4 \mathrm{E}\right)$, ne conserve une colonne vertébrale intacte et le processus épineux de ses vertèbres thoraciques est souvent détruit en partie ou en totalité.

387 Reshetov (1974) a aussi montré que chez B. bonasus la longueur des membres était plus grande par rapport à la longueur du corps dans sa partie correspondant aux vertèbres dorsales que chez B. bison. Là encore les squelettes d'Habarra ne fournissent pas d'éléments de comparaison.

Les auteurs ont naturellement mis en avant la stature plus imposante de B. priscus et l'envergure beaucoup plus grande de ses cornes. Il faut y ajouter, comme notamment Skinner et Kaisen (1947, p. 161, 213 et 229) l'ont fait, que chez B. bison et B. bonasus (fig. 28-1) la partie distale des processus cornuaux est très souvent affectée d'une torsion, plus ou moins accentuée, qui tend à diriger l'apex vers l'arrière. Rien de tel ne s'observe sur les chevilles osseuses suffisamment bien conservées ou reconstituables des bisons d'Habarra. On constate bien sur celles-ci l'ébauche d'une torsion mais elle semble n'avoir d'autre effet que de diriger l'apex vers l'avant. 
Figure 28 - Bisons. Cheville osseuse de corne et représentations paléolithiques diverses d'encornures.

Figure 28 - Twisted distal end of a bison horn core, and selected Paleolithic depictions of bison horns.

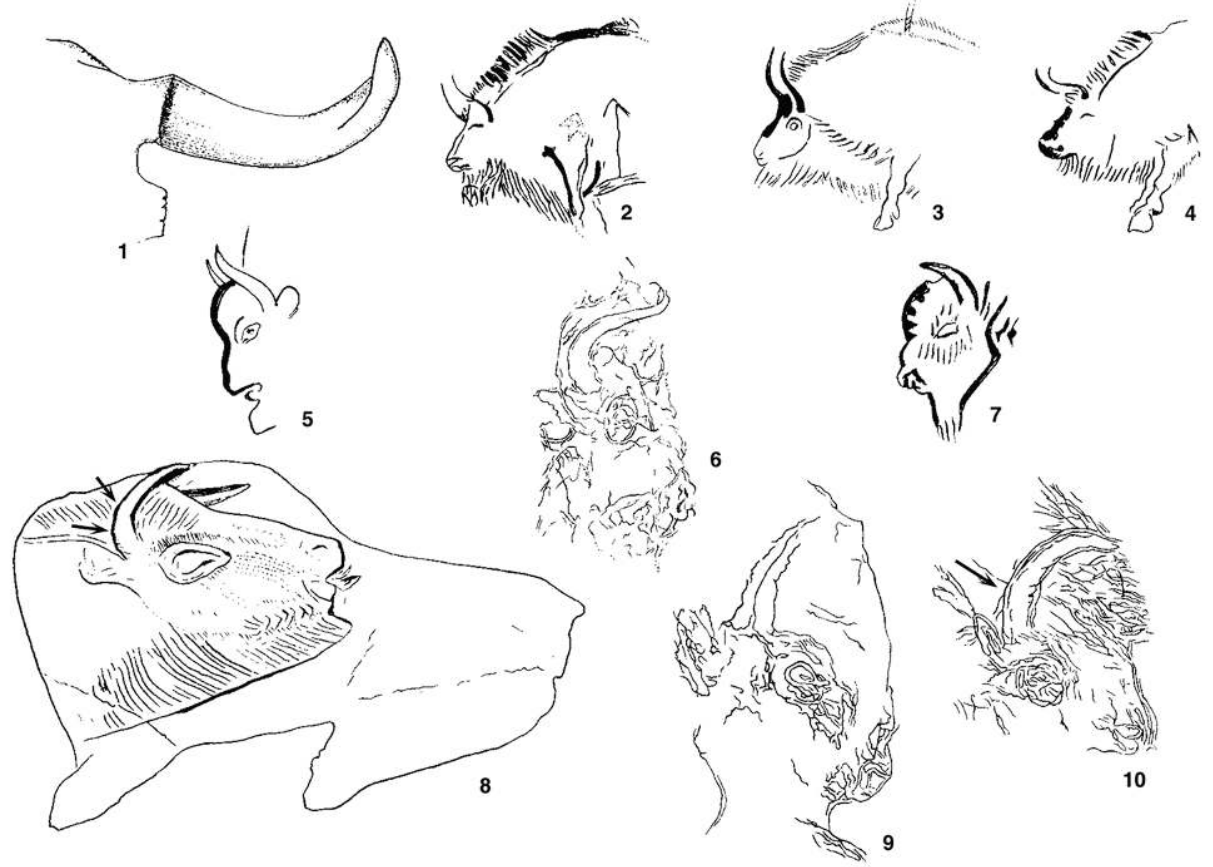

1 - Cheville osseuse de corne dont la région distale présente une torsion qui oriente l'apex vers l'arrière. Cette disposition commune chez $B$. bison et $B$. bonasus n'affecte pas les processus osseux, connus à ce jour, du Bison d'Habarra.

2, 3 (inversé) et 4 - Bisons du Salon noir de Niaux. D'après Henri Breuil $(1950,1952)$

6,9 et 10 - Têtes de Bison gravées de La Marche (Vienne). D'après Pales (1981, fig. 35)

7 - Bison gravé sur plaquette de la grotte de Raymonden à Chancelade (Dordogne). Musée du Périgord. D'après cliché Alain Roussot (in Sonneville-Bordes,1967, fig. 131).

8 - Bison sur bois de Renne. La Madeleine à Tursac (Dordogne). Musée des Antiquités Nationales.

D'après une photo des Archives photographiques (in Collectif, 1969).

L'examen des graphiques 9 à 12 qui expriment les rapports de longueur des grands os longs, déjà pratiqué pour la distinction des sexes chez le Bison, fait aussi apparaitre des différences entre espèces auxquelles nous n'accordons qu'un caractère hypothétique étant donné le nombre assez faible d'individus considérés. Nous les signalons néanmoins ci-après.

\begin{tabular}{|l|l|l|}
\hline & femelles (fig. 9) & mâles (fig. 10) \\
\hline $\begin{array}{l}\text { B. priscus } \\
\text { Habarra } \\
\text { comparé à } \\
\text { B. bonasus }\end{array}$ & $\begin{array}{l}\text { Les métatarses apparaissent moins courts par aux tibias chez B. priscus. En revanche ces } \\
\text { derniers seraient moins allongés par rapport aux } \\
\text { fémurs. }\end{array}$ & $\begin{array}{l}\text { Entre les deux espèces il n'existe } \\
\text { pas différences très nettes } \\
\text { autres que celles liées à la taille } \\
\text { des individus. }\end{array}$ \\
\hline & femelles (fig. 11) & mâles (fig. 12) \\
\hline
\end{tabular}




\begin{tabular}{|c|c|c|}
\hline $\begin{array}{l}\text { B. priscus } \\
\text { Habarra } \\
\text { comparé à } \\
\text { B. bison }\end{array}$ & $\begin{array}{l}\text { Chez B. priscus, les métacarpes seraient plus } \\
\text { courts par rapport aux radius, aux fémurs et aux } \\
\text { tibias et les métatarses le seraient par rapport } \\
\text { aux tibias et aux fémurs. On notera toutefois } \\
\text { que le tracé relatif au bison } 4 \mathrm{E} \text { d'Habarra, } \\
\text { à condition de faire abstraction des différences } \\
\text { liées à la stature particulièrement haute de ce } \\
\text { sujet, présente des analogies avec le tracé } \\
\text { correspondant au bison d'Amérique actuel M.P. } \\
\begin{array}{l}\text { 1898-261. Celui-ci se distingue surtout du } \\
\text { précédent par ses } \\
\text { proportionnellement plus courts. }\end{array}\end{array}$ & $\begin{array}{l}\text { Chez le sujet } \mathrm{n}^{\circ} 2 \mathrm{AB} \text { fossile les } \\
\text { métacarpes et métatarses sont } \\
\text { proportionnellement plus courts } \\
\text { par rapport aux tibias et aux } \\
\text { fémurs. }\end{array}$ \\
\hline
\end{tabular}

De l'ensemble de ces comparaisons deux points semblent se dégager plus nettement:

- chez B. bison les métapodes seraient relativement plus longs par rapport aux autres os longs que chez B. priscus et B. bonasus.

- chez B. bonasus les femelles se distingueraient des femelles des deux autres espèces par leurs métatarses plus courts par rapport aux tibias.

Conformément à ce que divers auteurs ont observé sur d'autres documents appartenant à la même espèce, les squelettes d'Habarra témoignent d'un fort dimorphisme sexuel qui, selon Guthrie (1990, p. 158 et 159), serait plus accentué chez B. priscus que chez les espèces actuelles.

A propos du squelette appendiculaire nous rappelons que l'examen des graphiques 9 à 12 , déjà utilisés, révèle chez le Bison d'Habarra, comme chez B. bonasus et B. bison, des différences concernant les rapports de longueur métapode-stylopode et, surtout, métapode-zeugopode (pl. 5 et 6 ). Chez les femelles ( $1 \mathrm{AB}, 4 \mathrm{E}$ et $5 \mathrm{E})$ métacarpes et métatarses sont proportionnellement plus longs que chez le mâle 2AB. L'exemple suivant est très significatif. Chez la femelle $4 \mathrm{E}$ la longueur de l'humérus (au sommet de la tête articulaire) égale $333,5 \mathrm{~mm}$, celle du radius $355,5 \mathrm{~mm}$ et celle du métacarpe 232 $\mathrm{mm}$. Chez le mâle $2 \mathrm{AB}$ les mesures correspondantes atteignent respectivement $372 \mathrm{~mm}$, $378 \mathrm{~mm}$ et $229 \mathrm{~mm}$.

Le dimorphisme sexuel se manifeste aussi, et de façon particulièrement nette, dans la morphologie du crâne. Chez les femelles celui-ci est plus petit et plus étroit tandis que les reliefs d'insertion de la région temporo-occipitale indiquent une puissance moins grande des muscles du cou et de la tête. Les chevilles osseuses de cornes, considérablement moins développées, sont aussi plus nettement dressées dès leur base. Chez les mâles, en effet, ces appendices, dans leur partie proximale, tout en s'écartant du plan sagittal, se dirigent vers l'arrière et le bas, comme ployant sous leur propre poids ( $\mathrm{pl} .2$ et 3). Cependant, chez les premières comme chez les seconds, chaque processus cornual présente une courbure principale médiale (plus ou moins accentuée) qui, en vue frontale, détermine la forme générique dite "entre parenthèses » de l'encornure (pl. 1), tandis que la torsion distale conduisant la pointe en direction caudale, commune chez les bisons actuels, n'existerait pas. Or, sur la majorité des représentations paléolithiques du genre, pariétales et sur objets mobiliers, on constate que la corne, dessinée de profil, après avoir suivi le mouvement de l'axe osseux vers l'avant, tel que l'indiquent les pièces fossiles, finit par s'orienter vers l'arrière (fig. 28-2 
à 6). L. Pales (1981), exposant ses vues sur ce problème, note justement (p. 91) que l'étui corné qui se prolonge au-delà de la pointe de la cheville pouvait bien «dessiner des courbures que le mandrin osseux ne laisse pas présager ». Chez le Chamois, les cornes (stricto sensu) franchement coudées vers l'arrière à leur sommet ne recouvrent-elles pas chacune un appendice crânien rectiligne?

Mais il est également vrai que quelques bisons préhistoriques, peints ou gravés, montrent des cornes moins sinueuses (en lame de sabre), entièrement recourbées vers l'avant. C'est le cas du bison se léchant le flanc de La Madeleine (Dordogne) (fig. 28-8) et de celui de Raymonden à Chancelade (Dordogne) gravé sur pendeloque (fig. 28-7). Deux têtes gravées de La Marche (Vienne) (fig. 28-9 et 10), publiées par Pales (loc. cit., fig. 35$\mathrm{A}$ et B), présentent aussi ce même caractère. Pour Pales de telles figurations traduiraient l'intention de l'artiste de présenter le segment latéral de la corne qui, en profil absolu, serait invisible. Les limites de ce segment, à peu près vertical sur le dessin, seraient perceptibles (fig. 28-8 et 10, voir flèches). Plus haut la corne se dirige régulièrement vers l'avant. Selon Pales (p. 106) ce souci de « représenter, dans un seul plan, la corne en totalité a fait s'éloigner l'artiste de la réalité naturelle». Sans pour cela rejeter cette hypothèse nous constatons que ces «limites" marquées par des inflexions du trait restent très discrètes (fig. 28-8 et 10) ou absentes (fig. 28-7). Nous nous demandons en quoi la réalisation de ce segment latéral, indiqué de façon aussi peu appréciable, pouvait empêcher des dessinateurs aussi habiles que l'auteur du bison de La Madeleine et celui (ou ceux) des bisons de La Marche de terminer le contour de la corne en lui donnant sa forme réelle. Ne peut-on imaginer chez Bison priscus l'existence d'individus ne possédant pas la morphologie typique, comme cela se voit chez les espèces actuelles? Il serait bien étonnant que la relative versatilité des caractères spécifiques ait épargné cette partie du corps chez les formes pléistocènes.

\section{Remerciements}

Les auteurs remercient Marie-Françoise Bonifay, Chercheur honoraire du CNRS (France), et Donald K. Grayson, Professeur d'Anthropologie à l'Université de Washington (U.S.A.), pour leur relecture attentive et leurs suggestions qui ont contribué à l'amélioration de ce travail.

\section{Figures 3 à 26}


Figure 3 - Bison - tête osseuse, vues dorsale, nucale et ventrale ; emplacement des mesures effectuées.

Figure 3 - The bison skull in dorsal, nuchal, and ventral view, showing the location of measurements reported in the text.

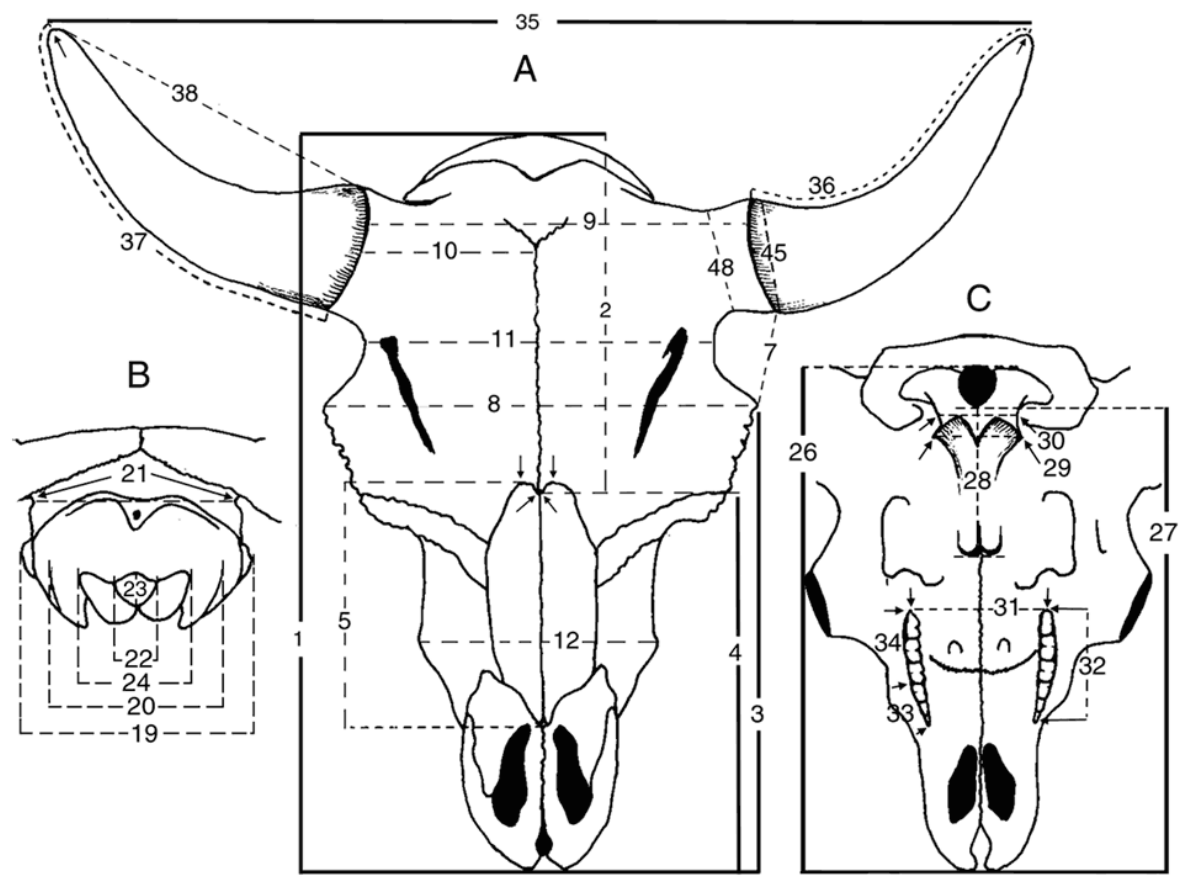


Les longueurs $(\mathrm{L})$ sont mesurées dans le plan sagittal ou parallèlement à celui-ci. Exceptions : mesures $7,32,34,36,37,38$ et 45 .

Les largeurs (I) sont mesurées perpendiculairement au plan sagittal

A - Vue dorsale

1 - (L) Distance entre la saillie nucale et la ligne touchant l'extrémité antérieure de chaque os incisif

2 - (L) Distance entre la saillie nucale et l'épine frontale

3 - (L) Distance entre la ligne joignant les bords postérieurs des orbites et la ligne touchant l'extrémité antérieure de chaque os incisif

4 - (L) Distance entre l'épine frontale et la ligne touchant l'extrémité antérieure de chaque os incisif

5 - (L) Longueur de l'os nasal

7 - (L) Longueur minimum entre le bord antérieur de la couronne du processus cornual et le bord postérieur de l'orbite

8 - (I) Largeur maximum au niveau des orbites

9 - (I) Largeur minimum entre le bord dorsal du processus cornual droit et son symétrique

10 - (I) Largeur minimum entre la ligne sagittale frontale et le bord dorsal du processus cornual

11 - (I) Largeur frontale minimum entre les orbites et les processus cornuaux

12 - (I) Largeur au niveau des tubercules faciaux

35 - Distance entre l'apex du processus cornual droit et l'apex du processus cornual gauche

36 - Longueur du processus cornual suivant la courbe supérieure

37 - Longueur du processus cornual suivant la courbe inférieure

38 - Longueur du processus cornual en ligne droite : distance minimum entre l'apex et le bord dorsal

de la couronne

45 - Diamètre antéro-postérieur de la couronne du processus cornual

48 - Diamètre antéro-postérieur minimum du col du processus cornual

La mesure $n^{\circ} 6$ n'apparaît pas dans cette liste. Elle correspond à la largeur maximum de l'os nasal. Elle est prise perpendiculairement à l'axe principal de cet os.

D'autres indications de mesures concernant les processus cornuaux sont portées figures 24 et 25 .

B - Vue nucale

19 - (I) Largeur maximum au niveau des processus mastoïdes

20 - (I) Largeur maximum au niveau des processus jugulaires

21 - (I) Largeur minimum entre les lignes temporales

22 - (I) Largeur maximum du foramen magnum

23 - Hauteur du foramen magnum mesurée dans le plan sagittal

24 - (I) Largeur bicondylienne

$\mathrm{C}$ - Vue ventrale

26 - (L) Longueur condylo-basale

27 - (L) Longueur basale

28 - (L) Longueur de la partie basilaire de l'occipital

29 - (I) Largeur maximum du basioccipital au niveau des revers basilaires des condyles

30 - (I) Largeur minimum de la partie basilaire des condyles

31 - (I) Largeur du palais au niveau du bord alvéolaire distal des M3

32 - Longueur de la série P2-M3, du bord alvéolaire mésial de P2 au bord alvéolaire distal de M3

33 - Longueur de la série P2-P4 mesurée du côté vestibulaire

34 - Longueur de la série P4-M3 également mesurée du côté vestibulaire 
Figure 4 - Bison - tête osseuse, vue latérale ; mandibule vue latérale ; dents isolées ; emplacement des mesures effectuées ; usure des molaires en fonction du pouvoir abrasif des aliments.

Figure 4 - The bison skull and mandible in lateral view and isolated bison teeth, showing the location of measurements reported in the text and molar usewear as a function of dietary abrasion.

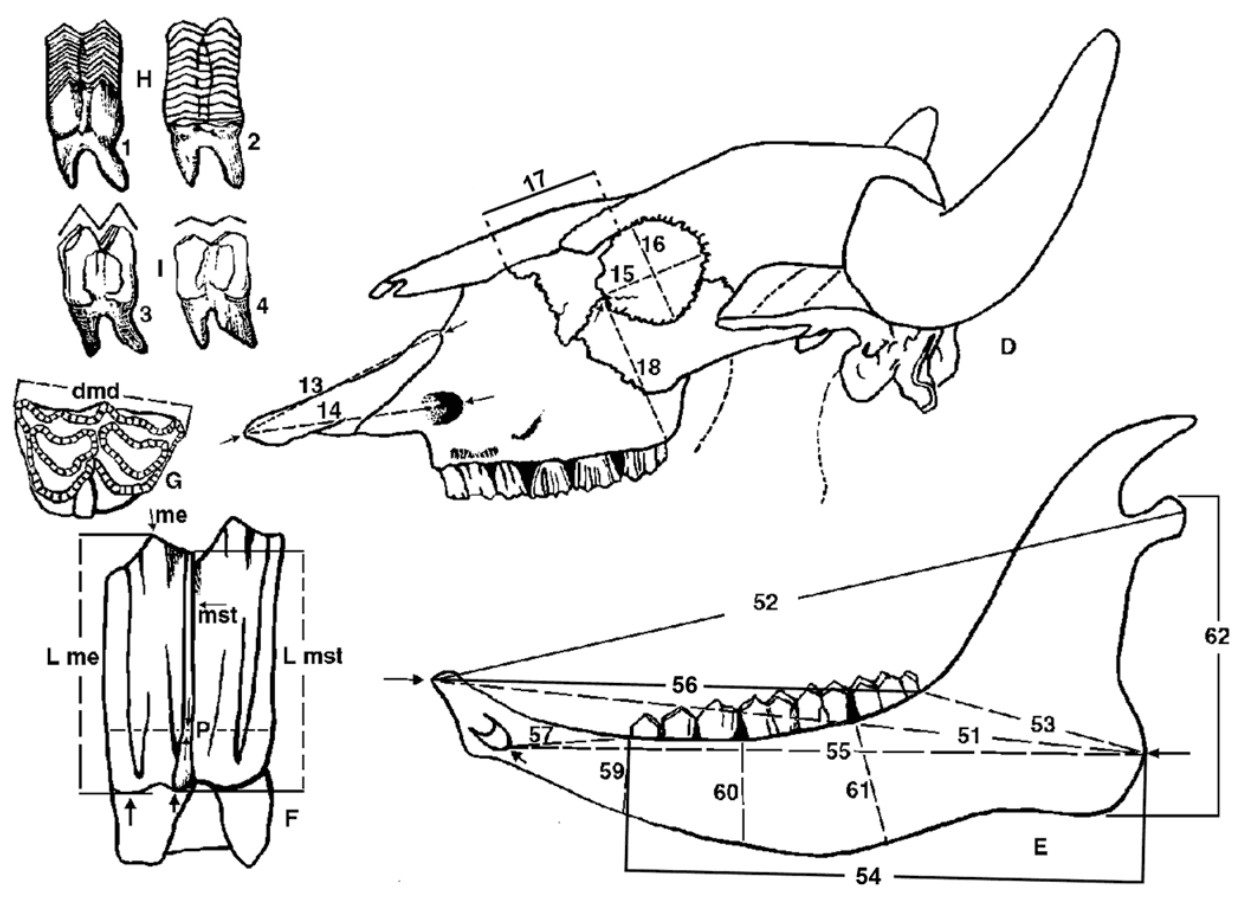


D - Vue latérale

13 - Longueur maximum de l'os incisif

14 - Distance maximum entre l'extrémité antérieure de l'os incisif et le bord postérieur du foramen infra-orbitaire

15 - Diamètre antéro-postérieur maximum de l'orbite mesuré à partir du point où la suture lacrymojugale rejoint le bord de l'orbite

16 - Diamètre maximum de l'orbite perpendiculaire au précédent (15)

17 - Longueur maximum de l'os lacrymal, du point le plus oral au point le plus aboral

18 - Distance du bord alvéolaire distal de M3 au point où la suture lacrymo-jugale recoupe le bord de l'orbite

E - Mandibule, vue latérale

51 - Distance entre le point le plus oral de la symphyse et le point le plus aboral de l'angle mandibulaire

52 - Distance entre le point le plus oral de la symphyse et le point le plus aboral du condyle

53 - Distance entre le bord alvéolaire distal de M3 et le point le plus aboral de l'angle mandibulaire

54 - Distance entre le bord alvéolaire mésial de P2 et le point le plus aboral de l'angle mandibulaire

55 - Distance entre le bord distal du foramen mentonnier et le point le plus aboral de l'angle mandibulaire

56 - Distance entre le point le plus oral de la symphyse et le bord alvéolaire distal de M3

57 - Distance entre le bord distal du foramen mentonnier et le bord alvéolaire mésial de P2

58 - Longueur du diastème séparant le bord alvéolaire distal de la canine du bord alvéolaire mésial de P2 (non situé sur le schéma)

59 - Hauteur du corps au niveau du bord alvéolaire mésial de P2

60 - Hauteur du corps au niveau du bord alvéolaire mésial de M1

61 - Hauteur du corps au niveau du bord alvéolaire distal de M3

62 - Hauteur du bord ventral de l'angle mandibulaire au sommet du condyle

F - Molaire supérieure gauche. Face vestibulaire

me - métacône L me - Longueur du métacône

mst - mésostyle L mst - Longueur du mésostyle

$\mathrm{P}$ - point situé sur le mésostyle à $1 \mathrm{~cm}$ du « départ » de celui-ci. A son niveau peuvent être mesurés les diamètres mésio-distal et vestibulo-lingual

G - Molaire supérieure gauche. Face occlusale

$\mathrm{d}$ m-d - diamètre mésio-distal

$\mathrm{H}$ - Usure des molaires en fonction du pouvoir abrasif des aliments (d'après Guthrie, 1990, p. 181, fig 7.3

1 - M1 inférieure. Usure relativement lente. Bison du Wood Buffalo Park se nourrissant

essentiellement de Carex.

2 - M1 inférieure. Usure plus rapide en relation avec une nourriture essentiellement herbacée. Bison

des Grandes Plaines.

I - Reliefs de la face occlusale (d'après Guthrie, 1990, p. 181, fig. 7.3.)

3 - Caractère aigu des cuspides d'une M1 inférieure à usure lente

4 - Caractère peu aigu des cuspides d'une molaire à usure rapide : la face occlusale tend à s'aplanir. 
Figure 5 - Bison, scapula, os coxal, troisième phalange ; emplacement des mesures effectuées. Figure 5 - The bison scapula, innominate, and third phalanx, showing the location of measurements reported in the text.

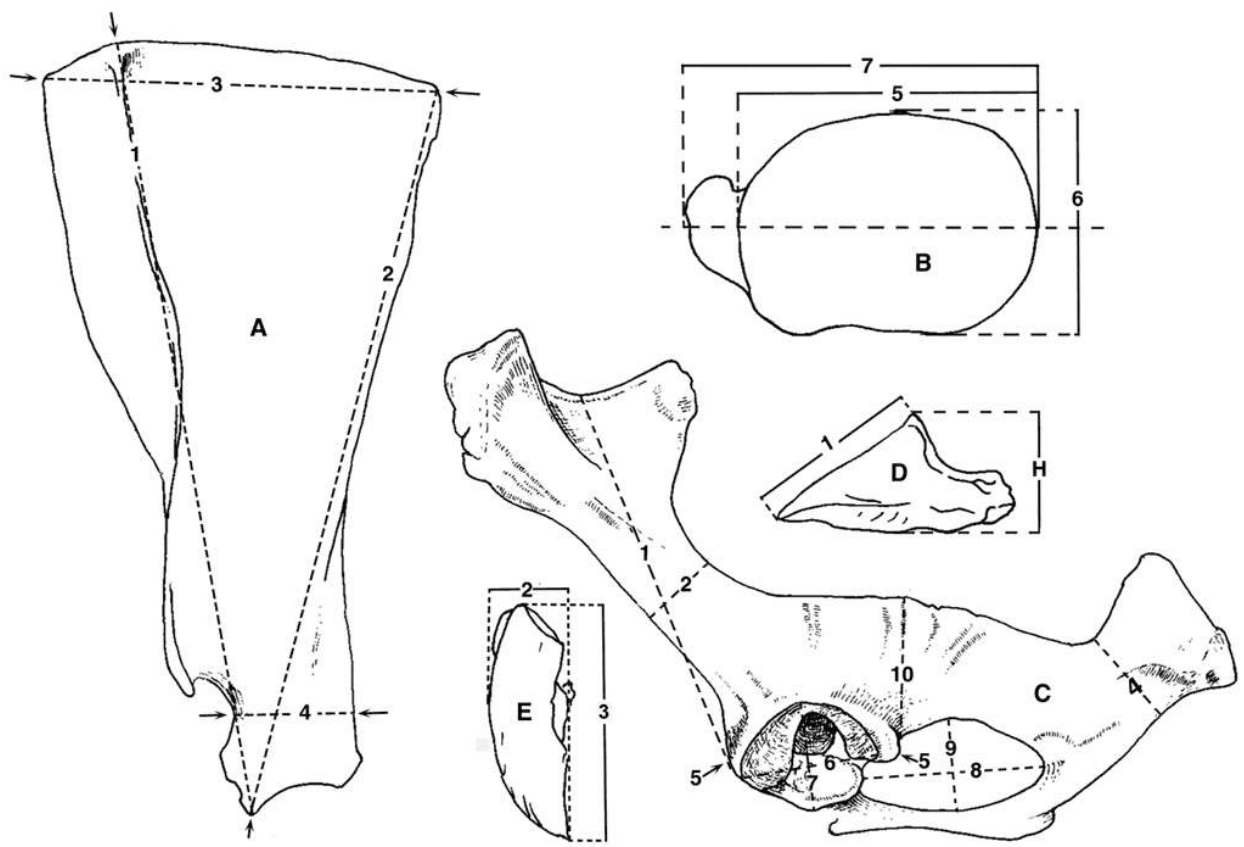

A - Scapula gauche, face latérale

1 - Longueur du point le plus crânial de la cavité glénoïde au point le plus dorsal de l'épine scapulaire

2 - Longueur du point le plus crânial de la cavité glénoïde au point le plus caudal du bord dorsal de l'os

3 - Largeur maximum au niveau du bord dorsal

4 - Largeur minimum du col

B - Scapula gauche, face ventrale

5 - Diamètre antéro-postérieur de la cavité glénoïde

6 - Diamètre transversal maximum de la cavité glénoïde

7 - Diamètre antéro-postérieur total de la région ventrale

C - Os coxal gauche, face latérale (d'après Barone, 1986, p. 592)

1 - Distance entre le point le plus crânial de l'acétabulum et le point correspondant au maximum de la concavité de la crête iliaque

2 - Largeur minimum du col de l'ilium

4 - Largeur minimum de la table de l'ischium

5 - Diamètre antéro-postérieur maximum de l'acétabulum, du rebord crânial au rebord caudal (5-5)

6 - Diamètre antéro-postérieur maximum de la surface semi-lunaire

7 - Diamètre dorso-ventral de l'acétabulum mesuré perpendiculairement au diamètre antéro-postérieur

(5)

8 - Plus grand diamètre du trou ovalaire

9 - Diamètre dorso-ventral maximum du trou ovalaire

10 - Distance entre le point le plus dorsal de la crête sciatique et le point le plus caudal du bord de

l'acétabulum (5)

D - Troisième phalange, vue latérale

1 - Diamètre antéro-postérieur dorsal, de l'apex au sommet de l'éminence pyramidale

$\mathrm{H}$ - Hauteur au sommet de l'épinence pyramidale, l'os reposant sur sa face palmaire (3ème antérieure)

ou plantaire (3ème postérieure)

E - Troisième phalange, face palmaire (3ème antérieure) ou plantaire (3ème postérieure)

1 - Diamètre antéro-postérieur

2 - Diamètre transversal (perpendiculairement au précédent (1)) 
Figure 6 - Bison, humérus, radius, ulna ; emplacement des mesures effectuées. Figure 6 - The bison humerus, radius, and ulna, showing the location of measurements reported in the text.
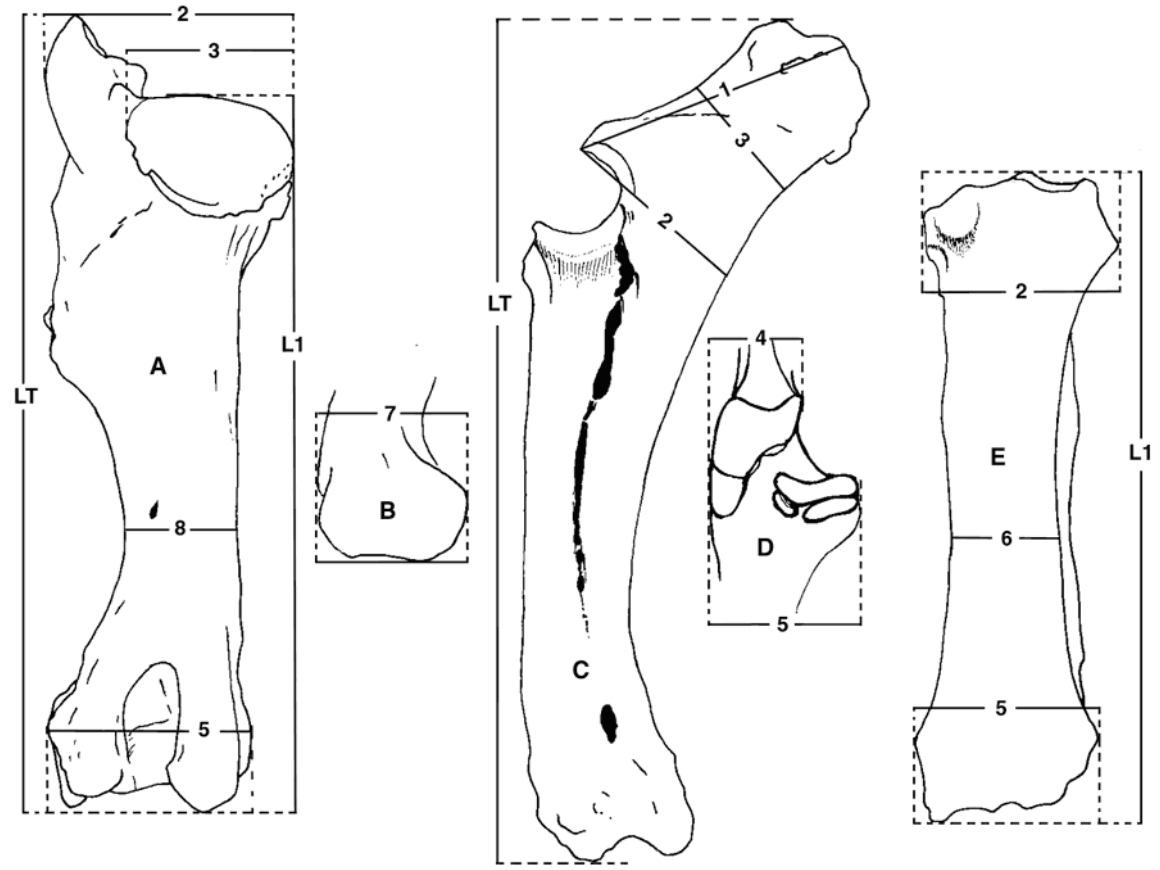

Les longueurs $(\mathrm{L})$ sont mesurées parallèlement à l'axe longitudinal de l'os et les largeurs (I) ou diamètres transversaux perpendiculairement à cet axe. Exceptions : mesures 1, 2 et 3 de la figure $5 \mathrm{C}$. A - Humérus gauche, face caudale (postérieure)

$\mathrm{LT}$ - Longueur $(\mathrm{L})$ totale

L1 - Longueur ( $\mathrm{L}$ ) mesurée du point le plus distal de l'épicondyle au sommet de la tête articulaire

2 - Diamètre transversal (I) proximal total

3 - Diamètre transversal (I) de la tête articulaire

5 - Diamètre transversal (I) distal total

8 - Diamètre transversal minimum (I minimum) de la diaphyse

B - Humérus gauche, face médiale

7 - Diamètre antéro-postérieur distal maximum de la face médiale au niveau de l'épicondyle médial

C - Radio-ulnaire gauche, face latérale

LT - Longueur totale mesurée parallèlement à l'axe longitudinal du radius

1 - Longueur de l'olécrâne, du bec au point diamétralement opposé

2 - Diamètre antéro-postérieur (I) maximum de l'olécrâne au niveau du bec

3 - Diamètre antéro-postérieur (I) minimum de l'olécrâne

D - Ulna gauche, face dorsale (antérieure)

4 - Diamètre transversal (I) de la face articulaire pour l'humérus

5 - Diamètre transversal (I)total au niveau de la face articulaire pour le radius

E - Radius gauche, face dorsale (antérieure)

L1 - Longueur totale $(\mathrm{L})$

2 - Diamètre transversal (I) proximal total

5 - Diamètre transversal (I) distal total

6 - Diamètre transversal (I) minimum de la diaphyse 
Figure 7 - Bovinés, fémur, tibia, talus ; emplacement des mesures effectuées.

Figure 7 - The bovine femur, tibia, and astragalus, showing the location of measurements reported in the text.
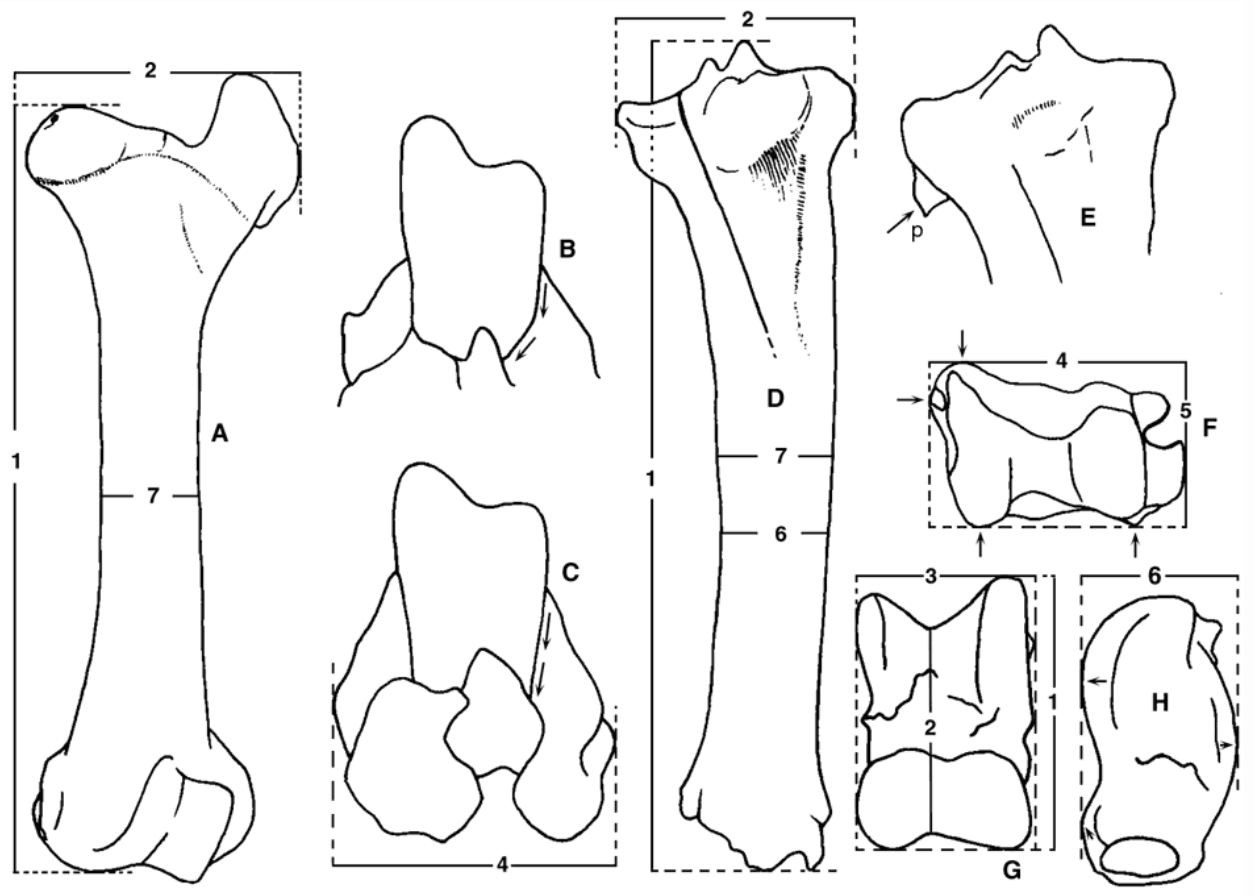

En $A, D$, G, les longueurs $(L)$ sont mesurées parallèlement à l'axe longitudinal de l'os et les largeurs (I) ou diamètres transversaux perpendiculairement à cet axe.

A - Fémur gauche, face crâniale (antérieure)

1 - Longueur ( $\mathrm{L}$ ) du sommet de la tête articulaire au point le plus distal de la lèvre médiale de la trochlée

2 - Diamètre transversal (I) proximal total

3 - Diamètre transversal (I) minimum de la diaphyse

B - Bos, fémur gauche, face distale

C - Bison, fémur gauche, face distale

4 - Largeur distale totale

D - Bison, Tibia droit, face crâniale (antérieure)

1 - Longueur totale $(\mathrm{L})$

2 - Diamètre transversal (I) proximal total

6 - Diamètre transversal (I) minimum de la diaphyse

7 - Diamètre transversal (I) au milieu de l'os

$\mathrm{E}$ - Bos, partie proximale de tibia droit avec vestige de péroné (p), face crâniale

$F$ - Tibia gauche, face distale

4 - Diamètre transversal (I) distal total

5 - Diamètre antéro-postérieur

G - Talus gauche, face dorsale (antérieure)

1 - Longueur totale $(\mathrm{L})$

2 - Longueur axiale

3 - Diamètre transversal (I) total

$\mathrm{H}$ - Talus gauche, face latérale

6 - Diamètre antéro-postérieur total 
Figure 8 - Bovinés, carpiens, métacarpe, première et deuxième phalanges ; emplacement des mesures effectuées.

Figure 8 - Bovine carpals, metacarpal, and first and second phalanges, showing the location of measurements reported in the text. 


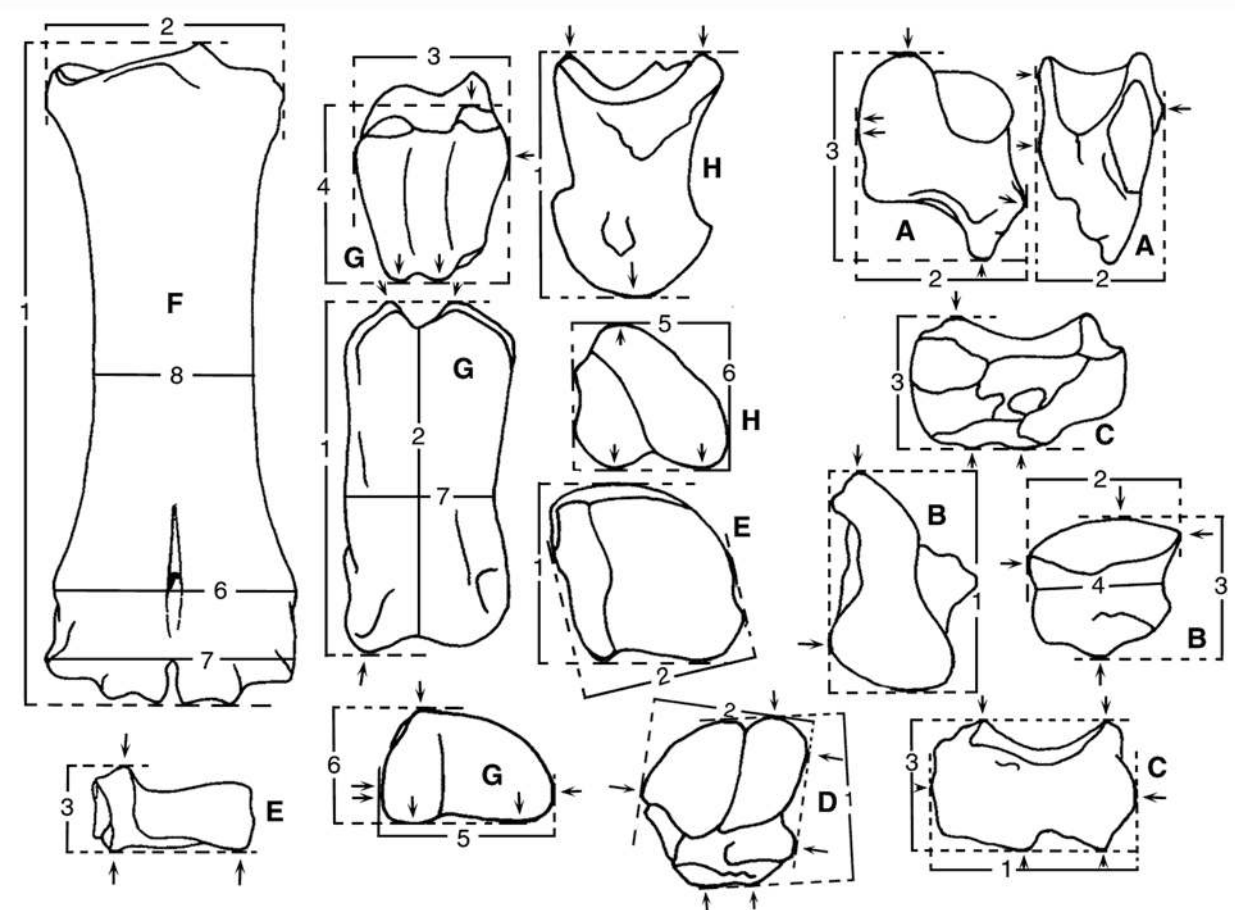

A - Pyramidal gauche

Vue latérale

1 - Diamètre antéro-postérieur

3 - Hauteur totale

Vue latéro-palmaire

2 - Diamètre transversal

$\mathrm{B}$ - Semi-lunaire gauche

Vue proximale

1 - Diamètre antéro-postérieur

Vue dorsale

2 - Diamètre transversal dorsal

3 - Hauteur dorsale ( voir aussi vue latérale)

4 - Diamètre transversal dorsal minimum

C - Scaphoïde droit

Vue médiale

1 - Diamètre antéro-postérieur

3 - Hauteur totale

D - Unciforme gauche

Vue proximale

1 - Diamètre antéro-postérieur

2 - Diamètre transversal

E - Capitato-trapézoïde gauche

Vue proximale

1 - Diamètre antéro-postérieur

2 - Diamètre transversal

Vue plantaire

3 - Hauteur latérale maximum

F - Métacarpe gauche

Vue dorsale

La longueur (L) est mesurée parallèlement à l'axe longitudinal de l'os et les largeurs (I) ou diamètres transversaux perpendiculairement à cet axe.

1 - (L) Longueur totale

2 - (I) Diamètre transversal proximal total

6 - (I) Diamètre transversal distal supra-articulaire

7 - (I) Diamètre transversal distal articulaire

8 - (I) Diamètre transversal minimum de la diaphyse. II diffère peu du diamètre transversal mesuré au milieu de l'os dans la plupart des cas

Les métatarses sont mesurés de la même façon que les métacarpes

G - Premières phalanges (antérieures et postérieures)

Les longueurs $(\mathrm{L})$ sont mesurées parallèlement à l'axe longitudinal de l'os et les largeurs (I) ou 
diamètres transversaux perpendiculairement à cet axe

Vue dorsale

1 - Longueur $(\mathrm{L})$ totale

2 - Longueur axiale

7 - Diamètre transversal (I) minimum de la diaphyse

Vue proximale

3 - Diamètre transversal proximal total

4 - Diamètre antéro-postérieur proximal total

Vue distale

5 - Diamètre transversal (I) distal articulaire

6 - Diamètre antéro-postérieur distal total

$\mathrm{H}$ - Deuxièmes phalanges (antérieures et postérieures)

Vue abaxiale

1 - Longueur totale : mesurée parallèlement à l'axe proximo-distal de l'os

Vue distale

5 - Diamètre transversal articulaire maximum

6 - Diamètre antéro-postérieur distal

Figure 9 - Bisons femelles. Bison priscus Habarra, Bison bonasus. Comparaison de la longueur relative des principaux os longs.

Figure 9 - A comparison of the relative lengths of the principal long bones of female Bison priscus Habarra and Bison bonasus.

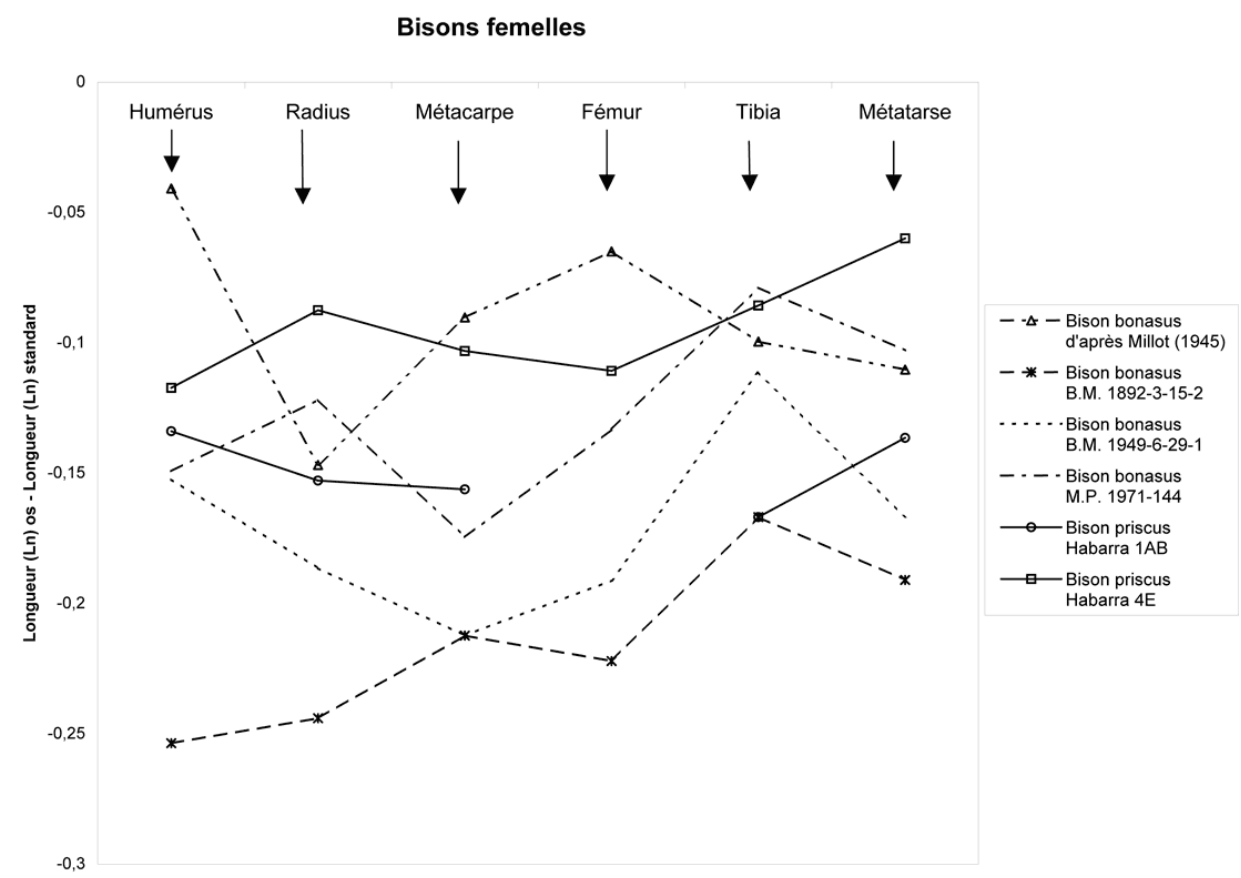

humérus $(H)$, radius $(R)$, métacarpe $(M c p)$, fémur $(F)$, tibia $(T)$, métatarse (Mts). Diagramme établi selon la méthode de Simpson. Référence 0 : Bos primigenius de la Prévalerie (Torsac, Charente) d'après J. Massaud (1984). Précisions : chapitre 4 (détermination du sexe) 
Figure 10 - Bisons mâles. Bison priscus Habarra, Bison bonasus. Comparaison de la longueur relative des principaux os longs.

Figure 10 - A comparison of the relative lengths of the principal long bones of male Bison priscus Habarra and Bison bonasus.

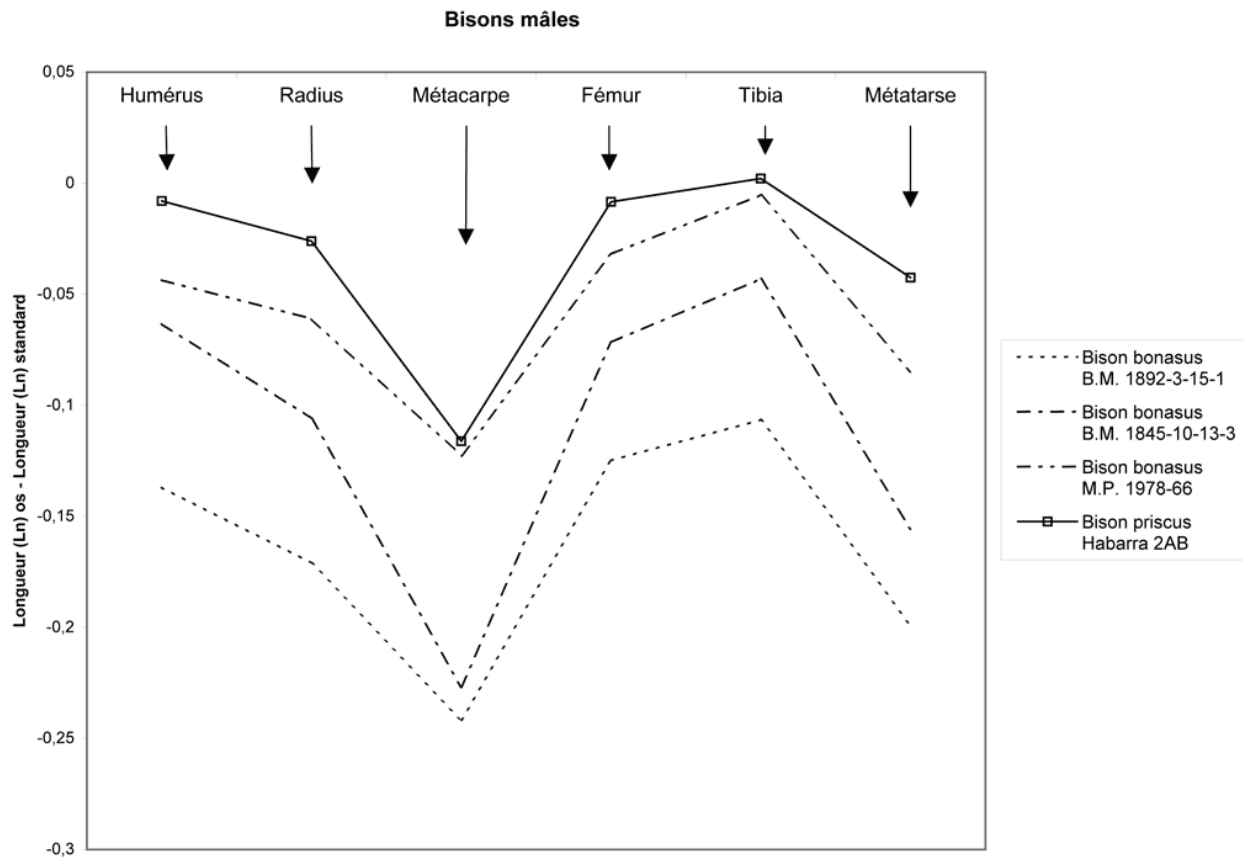

humérus $(H)$, radius $(R)$, métacarpe $(M c p)$ fémur $(F)$, tibia $(T)$, métatarse (Mts). Diagramme établi selon la méthode de Simpson. Référence 0 : Bos primigenius de la Prévalerie (Torsac, Charente) d'après J. Massaud (1984). Précisions : chapitre 4 (détermination du sexe) 
Figure 11 - Bisons femelles. Bison priscus Habarra, Bison bison. Comparaison de la longueur relative des principaux os longs.

Figure 11 - A comparison of the relative lengths of the principal long bones of female Bison priscus Habarra and Bison bison.

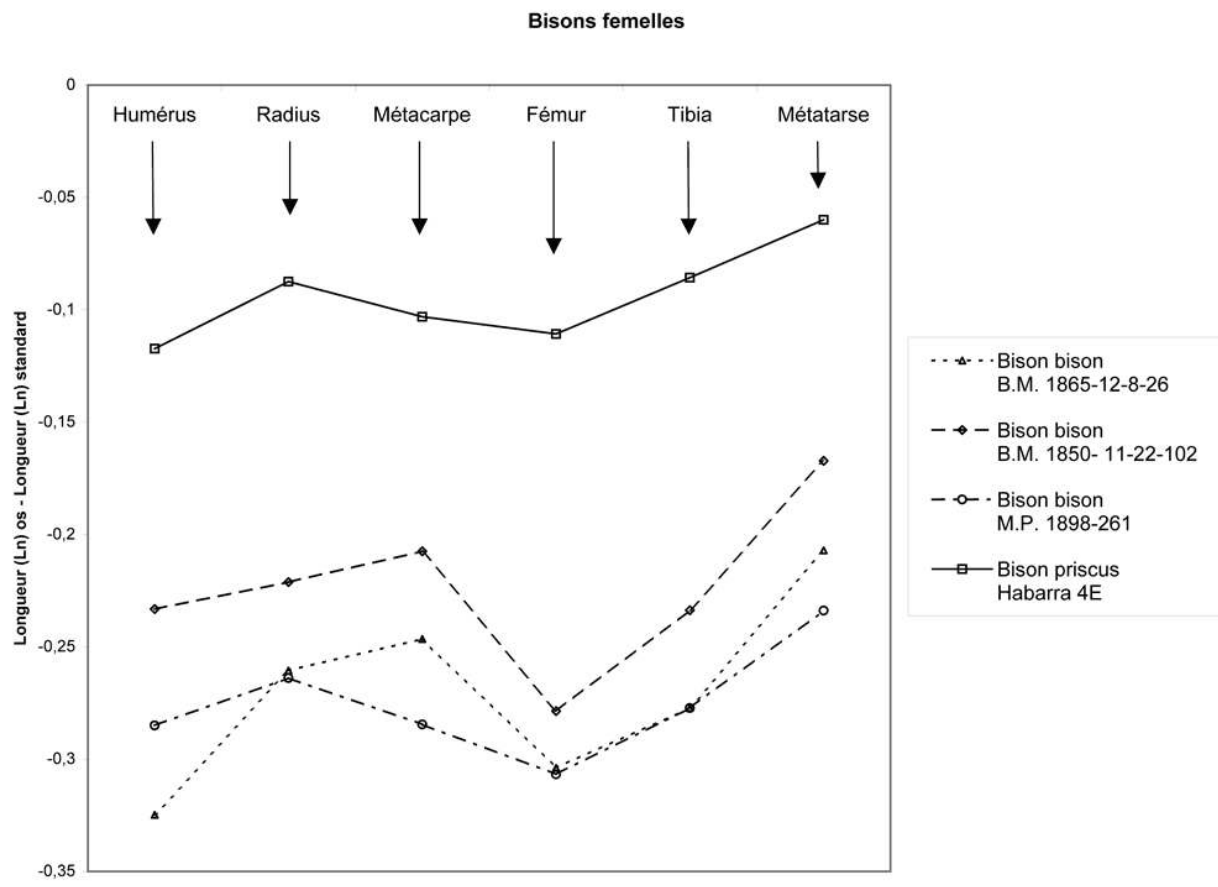

humérus $(H)$, radius $(R)$, métacarpe $(M c p)$ fémur $(F)$, tibia $(T)$, métatarse (Mts). Diagramme établi selon la méthode de Simpson. Référence 0 : Bos primigenius de la Prévalerie (Torsac, Charente) d'après J. Massaud (1984). Précisions : chapitre 4 (détermination du sexe) 
Figure 12 - Bisons mâles. Bison priscus Habarra, Bison bison. Comparaison de la longueur relative des principaux os longs.

Figure 12 - A comparison of the relative lengths of the principal long bones of male Bison priscus Habarra and Bison bison.

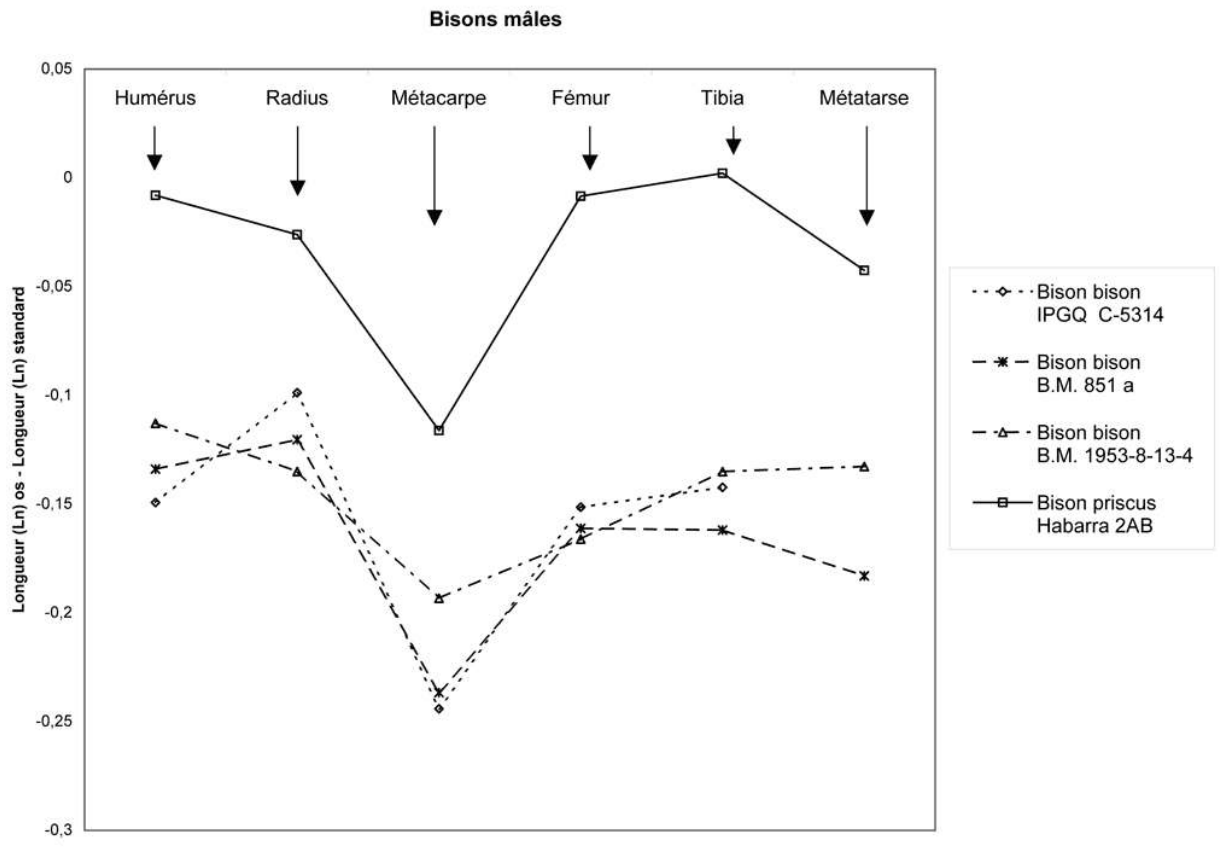

humérus $(H)$, radius $(R)$, métacarpe $(M c p)$ fémur $(F)$, tibia $(T)$, métatarse $(M t s)$. Diagramme établi selon la méthode de Simpson. Référence 0 : Bos primigenius de la Prévalerie (Torsac, Charente) d'après J. Massaud (1984). Précisions : chapitre 4 (détermination du sexe) 
Figure 13 - Bison priscus Habarra. Mâles et femelles. Comparaison de la longueur relative des principaux os longs.

Figure 13 - A comparison of the relative lengths of the principal long bones of male and female Bison priscus Habarra.

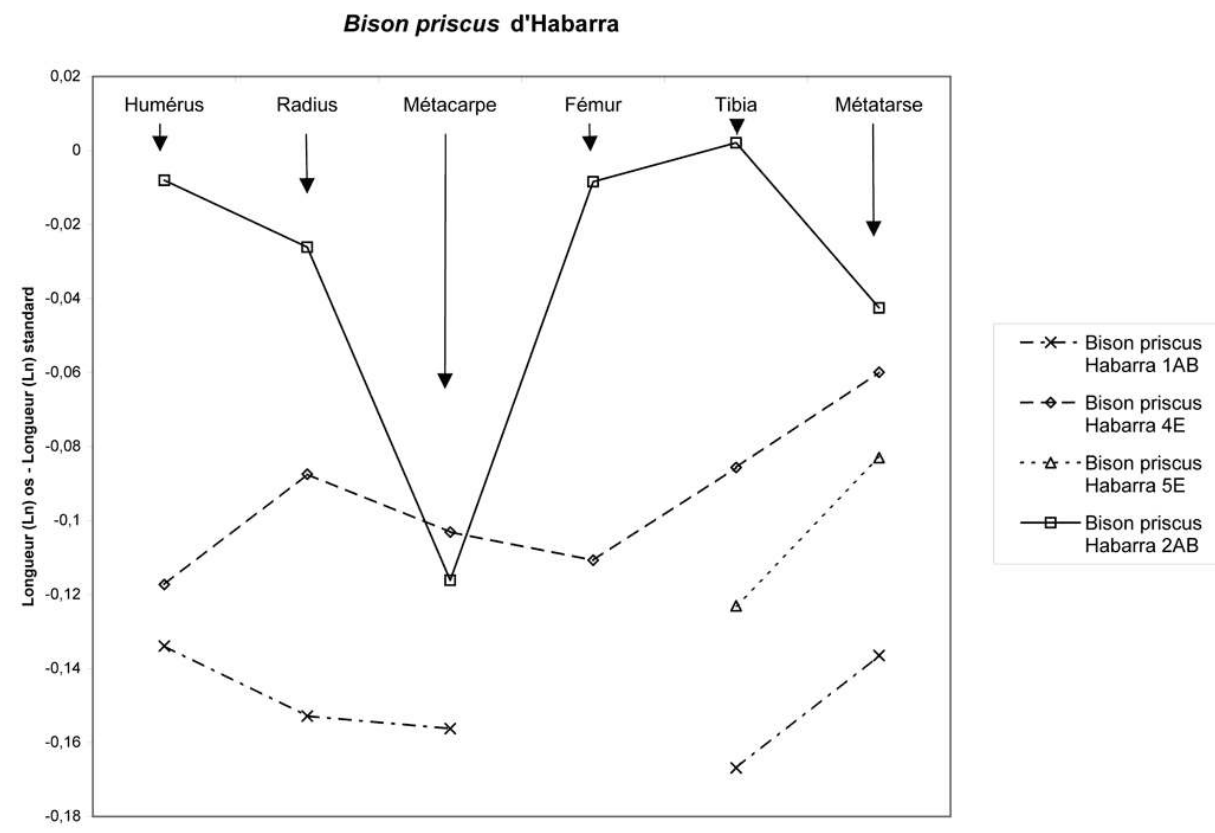

humérus $(H)$, radius $(R)$, métacarpe $(M c p)$ fémur $(F)$, tibia $(T)$, métatarse (Mts). Diagramme établi selon la méthode de Simpson. Référence 0 : Bos primigenius de la Prévalerie (Torsac, Charente) d'après J. Massaud (1984). Précisions : chapitre 4 (détermination du sexe) 
Figure 14 - Bos et Bison priscus Habarra. Humérus gauches. Extrémité distale. Face latérale. Figure 14 - Bos and Bison priscus Habarra: The distal extremity of the left humerus in lateral view.

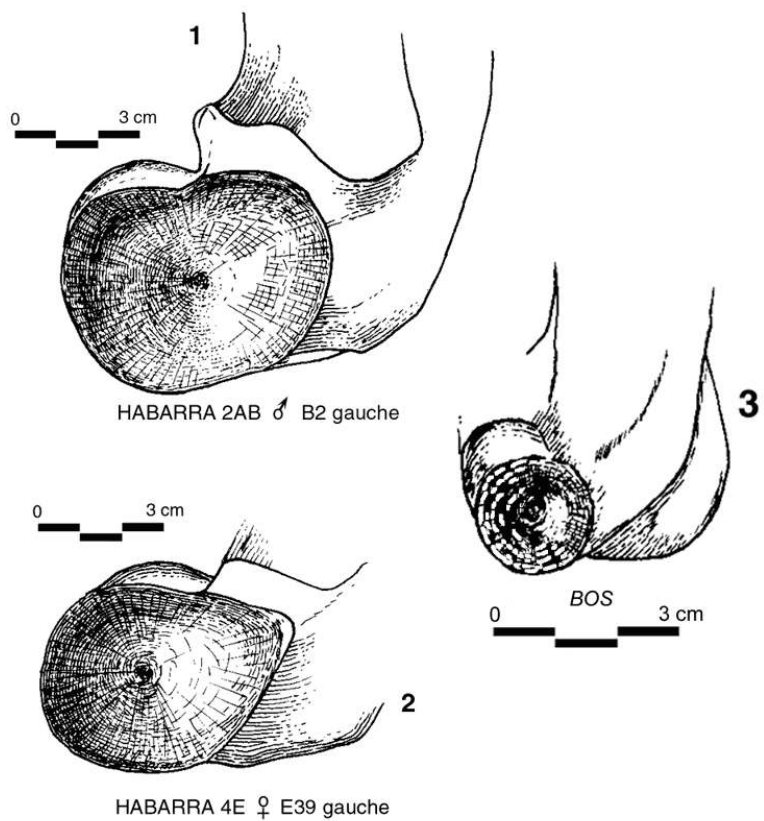

1 - Habarra. Bison priscus. Mâle $n^{\circ} 2 A B$

2 - Habarra. Bison priscus. Femelle n ${ }^{\circ} 4 \mathrm{E}$

3 - Bos (non à l'échelle) 
Figure 15 - Bos et Bison priscus Habarra. Radius. Extrémité proximale. Face dorsale. Figure 15 - Bos and Bison priscus Habarra: The proximal extremity of the radius in dorsal view.

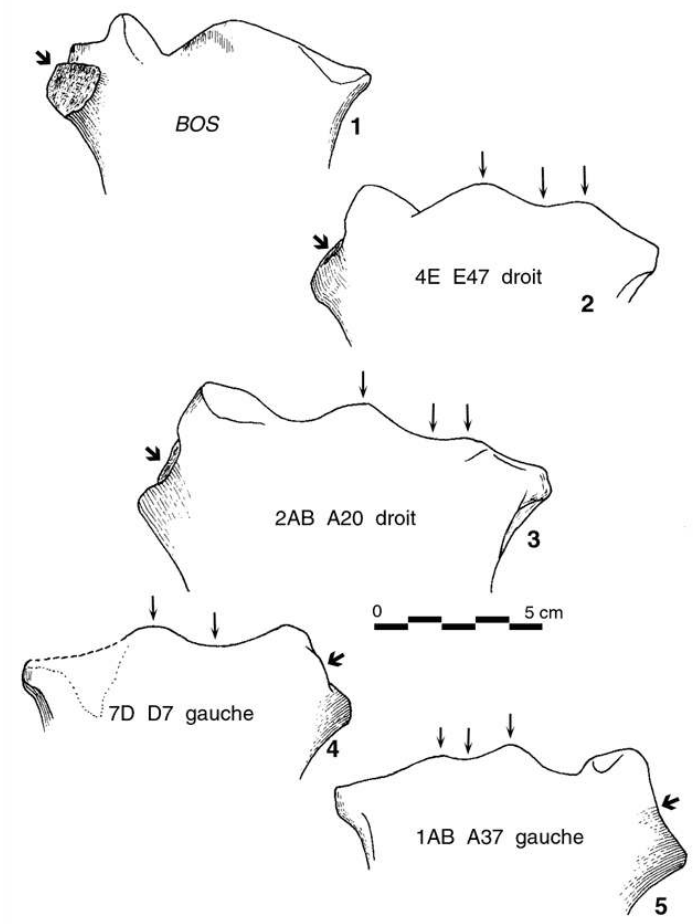

1 - Bos ; 2, 3, 4, 5 - Bison priscus. Habarra. Sur chaque dessin figurent la référence du squelette, celle de la pièce et sa latéralisation.

Au niveau du relief latéral d'insertion, la surface d'implantation du ligament collatéral (flèche épaisse) est nettement moins visible chez Bison que chez Bos, moins nettement orientée dorsalement. A noter le caractère sinueux du bord dorsal sur les quatre spécimens d'Habarra (petites flèches). 
Figure 16 - Bos et Bison priscus Habarra. Carpiens (Os pyramidal, semi-lunaire, scaphoïde, capitatotrapézoïde, unciforme) et tarsien (grand cunéiforme).

Figure 16 - Bos and Bison priscus Habarra: Carpals (pyramidal, semilunar, scaphoid, capitato-trapezoid, unciform) and tarsal (greater cuneiform).
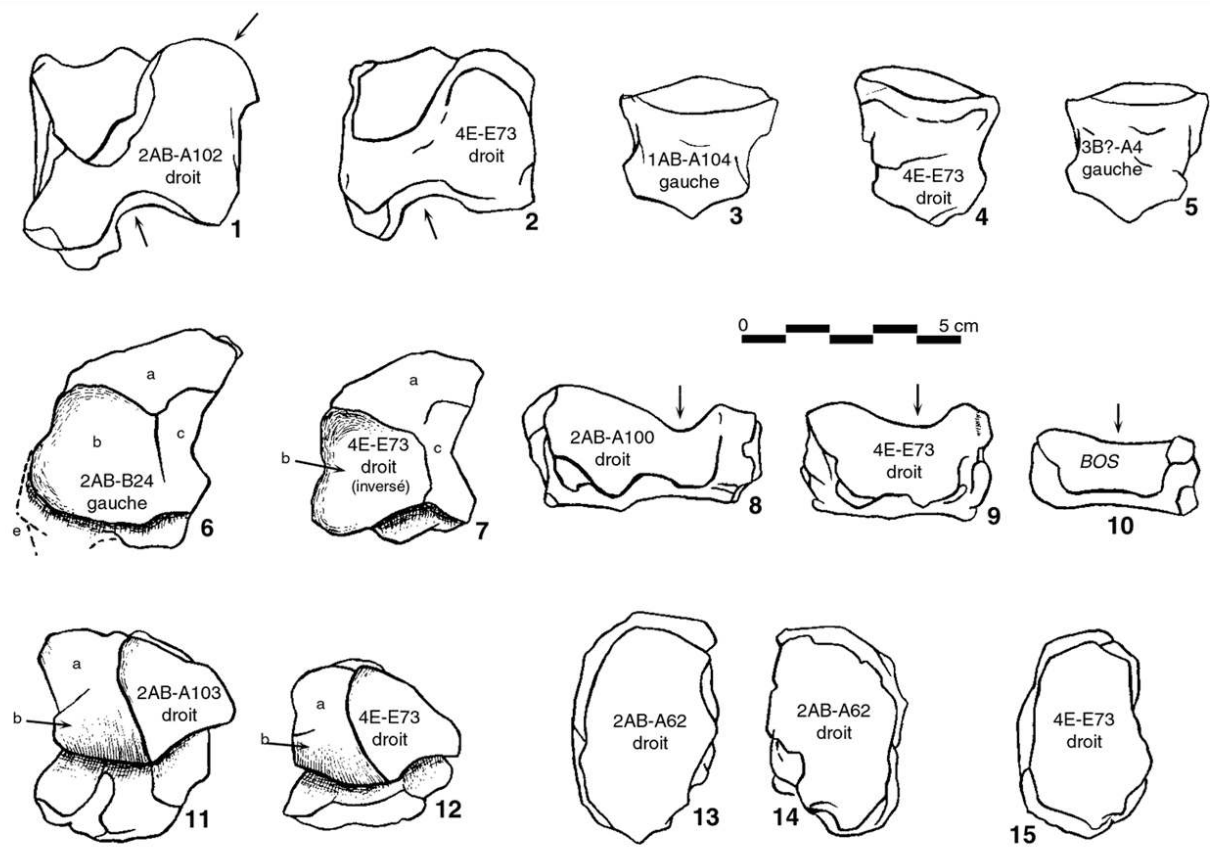

1 et 2 - Habarra. Bison priscus. Pyramidaux, face latérale

3,4 et 5 - Habarra. Bison priscus. Semi-lunaires, face dorsale (E73 droit inversé)

6 et 7 - Habarra. Bison priscus. Scaphoïdes, face distale

8 et 9 - Habarra. Bison priscus. Capitato-trapézoïdes, face palmaire

10 - Bos taurus. Capitato-trapézoïde, face palmaire

11 et 12 - Habarra. Bison priscus. Unciforme, face proximale

13 et 15 - Habarra. Bison priscus. Grand cunéiforme, face proximale

14 - Habarra. Bison priscus. Grand cunéiforme, face distale

Sur chaque dessin figurent la référence du squelette, celle de la pièce et sa latéralisation. 
Figure 17 - Bison priscus Habarra. Métacarpes. Face dorsale, face proximale. Figure 17 - Bison priscus Habarra : Metacarpals in dorsal and proximal view.
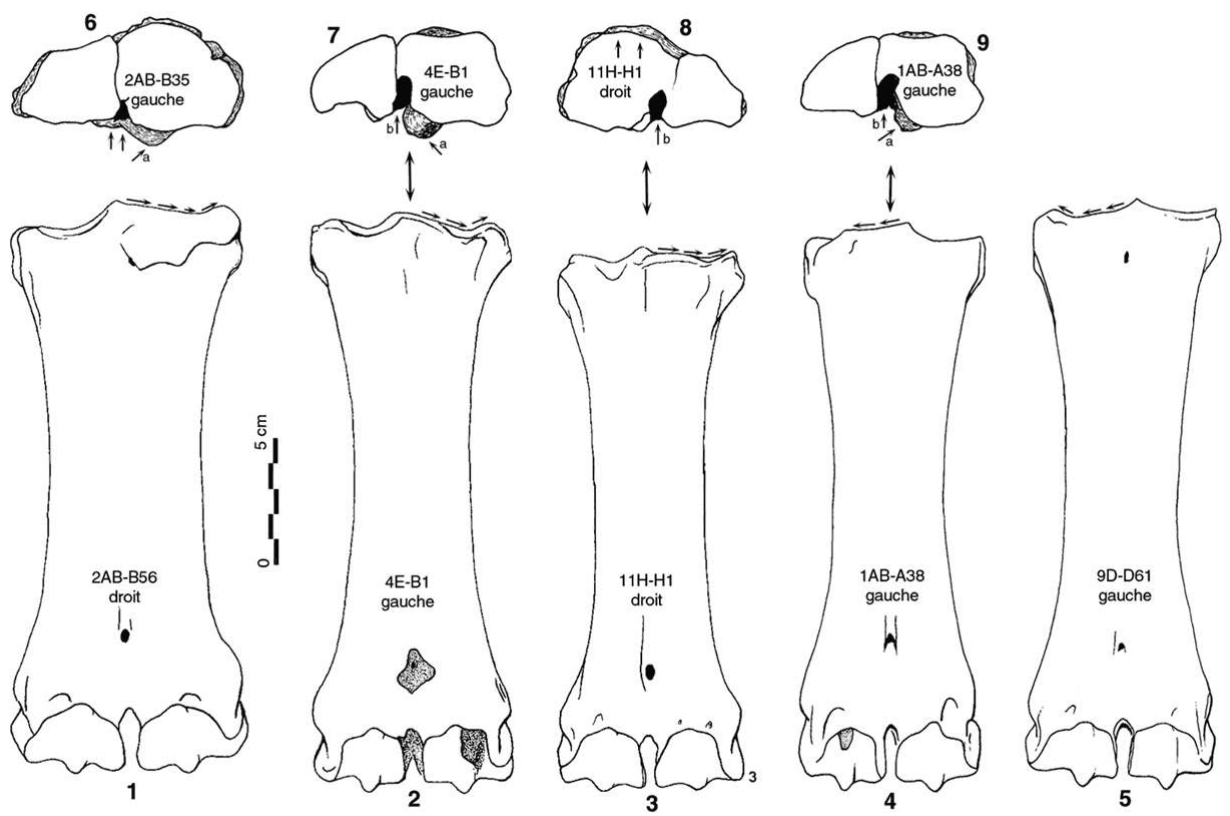

1 à 5 : face dorsale ; 1 - sujet mâle $2 A B ; 2$ - sujet femelle $4 \mathrm{E} ; 3$ - sujet femelle $11 \mathrm{H} ; 4$ - sujet femelle $1 A B ; 5$ - sujet femelle $9 D$

6 à 9 : face articulaire proximale ; 6 - sujet mâle $2 A B ; 7,8,9$ - sujets femelles $4 \mathrm{E}, 11 \mathrm{H}, 1 \mathrm{AB}$

$a$ : reliefs d'insertion musculaire; $b$ : fossette synoviale.

Sur chaque dessin figurent la référence du squelette, celle de la pièce et sa latéralisation. 
Figure 18 - Bos et Bison priscus Habarra. Premières phalanges antérieures et postérieures. Figure 18 - Bos and Bison priscus Habarra: First phalanges of the fore- and hind-limbs.
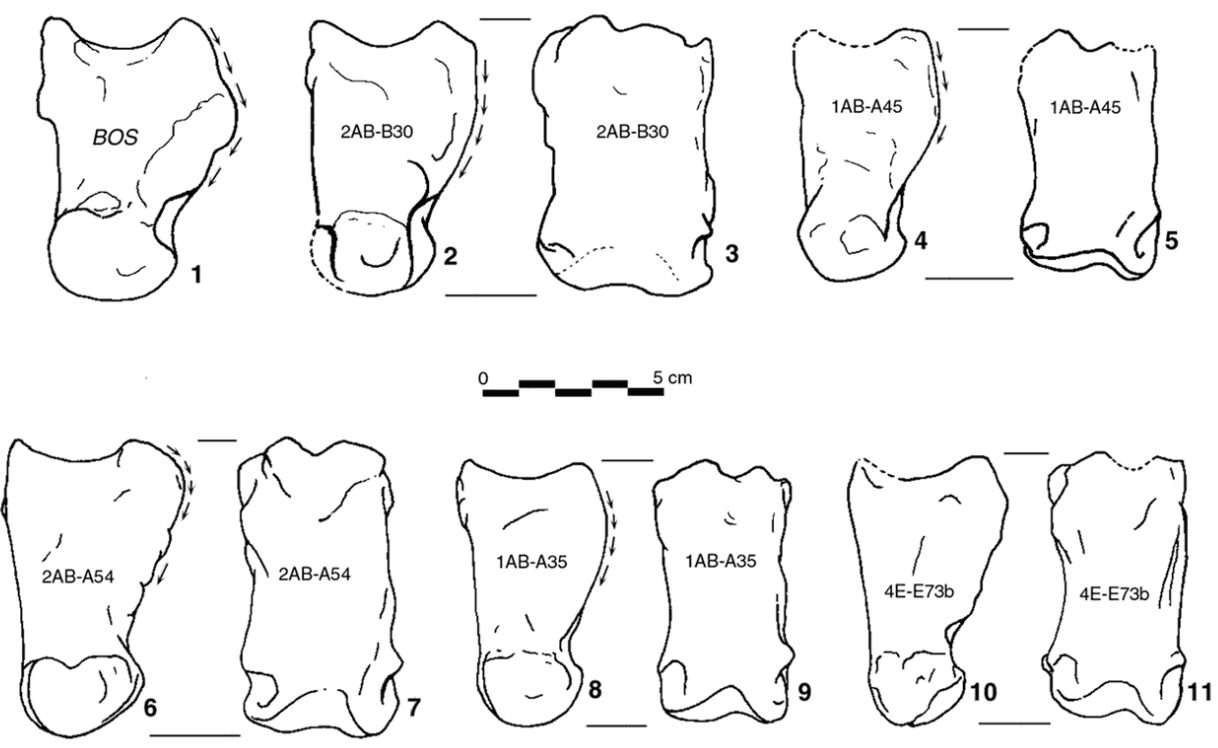

1 à 5 - phalanges antérieures; 1,2 et 3 face axiale ; 3 et 5 face dorsale 6 à 11 - phalanges postérieures; 6,8 et 10, face axiale ; 7, 9 et 11, face dorsale.

Sur chaque dessin figurent la référence du squelette et celle de la pièce. Tous les os représentés se rapportent au Bison d'Habarra (B. priscus) sauf le $n^{\circ} 1$ qui appartient au genre Bos.

Figure 19 - Bison priscus Habarra. Deuxièmes phalanges.

Figure 19 - Bison priscus Habarra : Second phalanges.
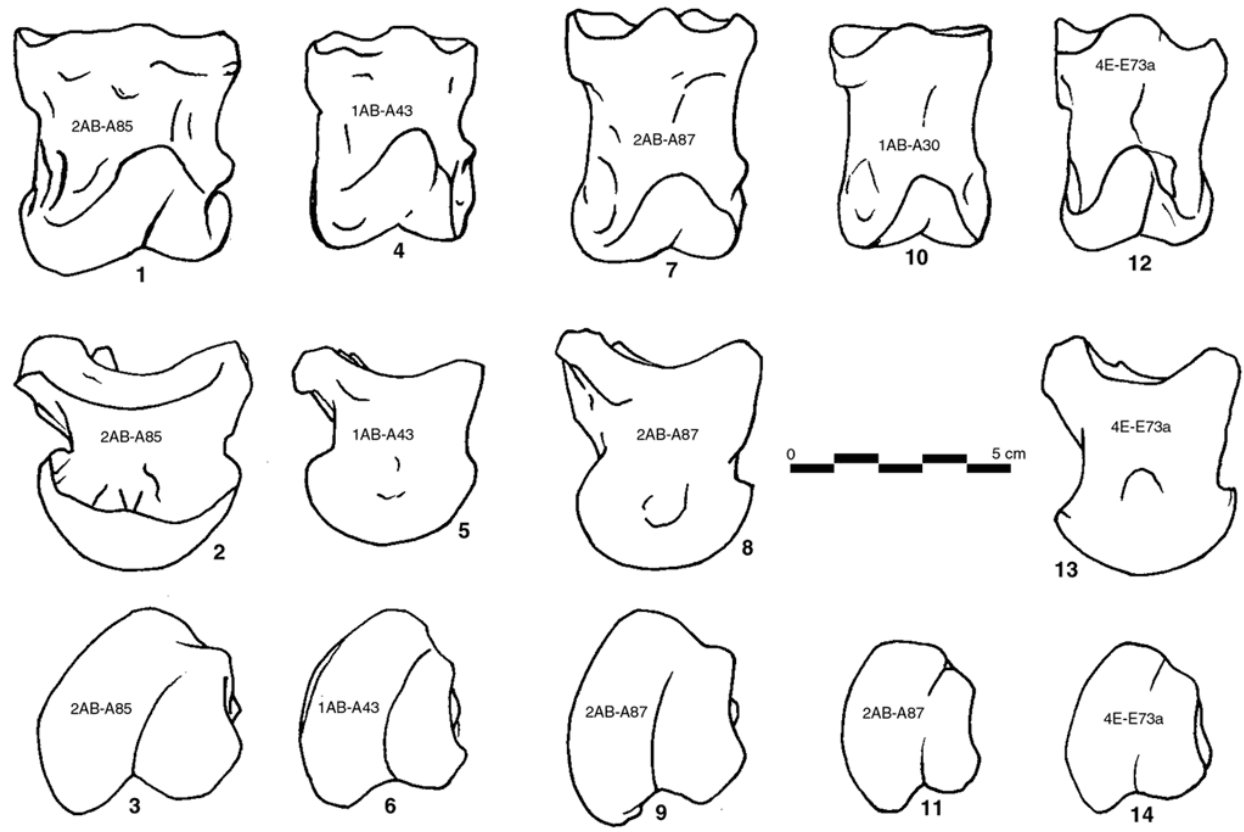

1 à 6 - phalanges antérieures ; 1 et 4, face dorsale ; 2 et 5 , face abaxiale ; 3 et 6 , face distale 7 à 14 - phalanges postérieures ; 7,10 et 12, face dorsale ; 8 et 13, face abaxiale ; 9,11 et 14, face distale

Sur chaque dessin figurent la référence du squelette et celle de la pièce. 
Figure 20 - Bison priscus Habarra. Tibias, face caudale ; morphologie des reliefs d'insertion musculaire.

Figure 20 - Bison priscus Habarra: Morphology of the muscle attachment ridges on the posterior face of the tibia.
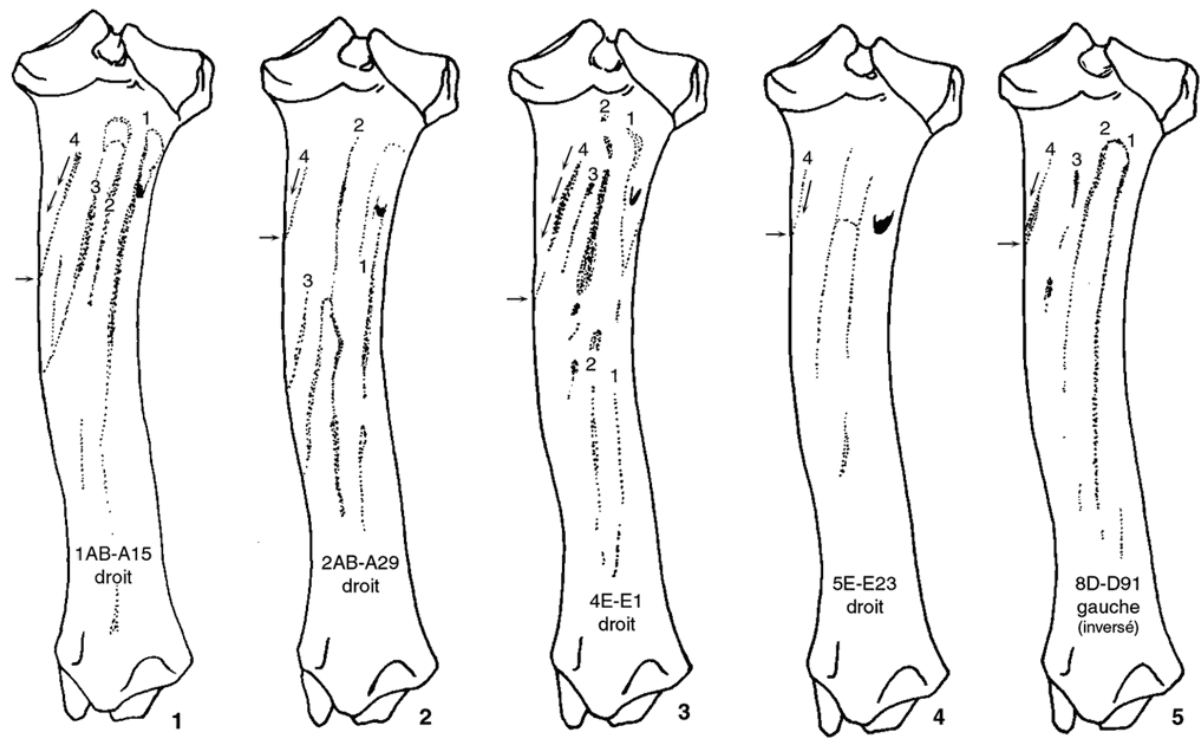

1 - tibia droit A15 du bison femelle $1 A B ; 2$ - tibia droit A29 du bison mâle 2AB ; 3 - tibia droit E1 du bison femelle 4E; 4 - tibia droit E23 du bison femelle $5 \mathrm{E} ; 5$ - tibia gauche (inversé) D91 du bison mâle 8D.

Obliquité relativement accusée des crêtes d'insertion musculaire $n^{\circ} 4$ et $n^{\circ} 3$ dans les sens latéromédial et proximo-distal. 
Figure 21 - Bos et Bison bison. Tibias, face caudale ; morphologie des reliefs d'insertion musculaire. Figure 21 - Bos and Bison bison: Morphology of the muscle attachment ridges on the posterior face of the tibia.
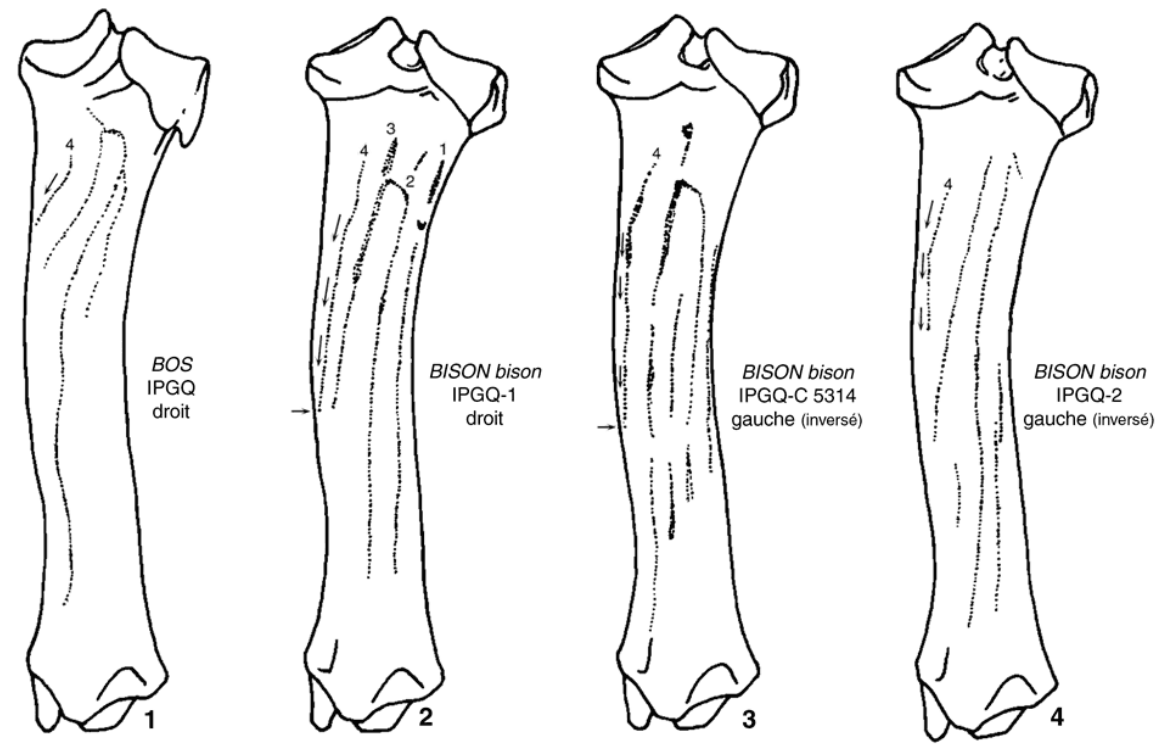

1 - Bos tibia droit ; 2 - Bison bison tibia droit ; 3 - Bison bison tibia gauche inversé ; 4 - Bison bison tibia gauche inversé.

Les flèches accompagnent la crête d'insertion musculaire $n^{\circ} 4$. Os de la collection de l'Institut de Préhistoire et Géologie du Quaternaire de l'Université Bordeaux I.

Figure 22 - Bovinés. Vertèbre thoracique et sacrum ; emplacement des mesures effectuées. Figure 22 - The bovine thoracic vertebra and sacrum, showing the location of measurements reported in the text.
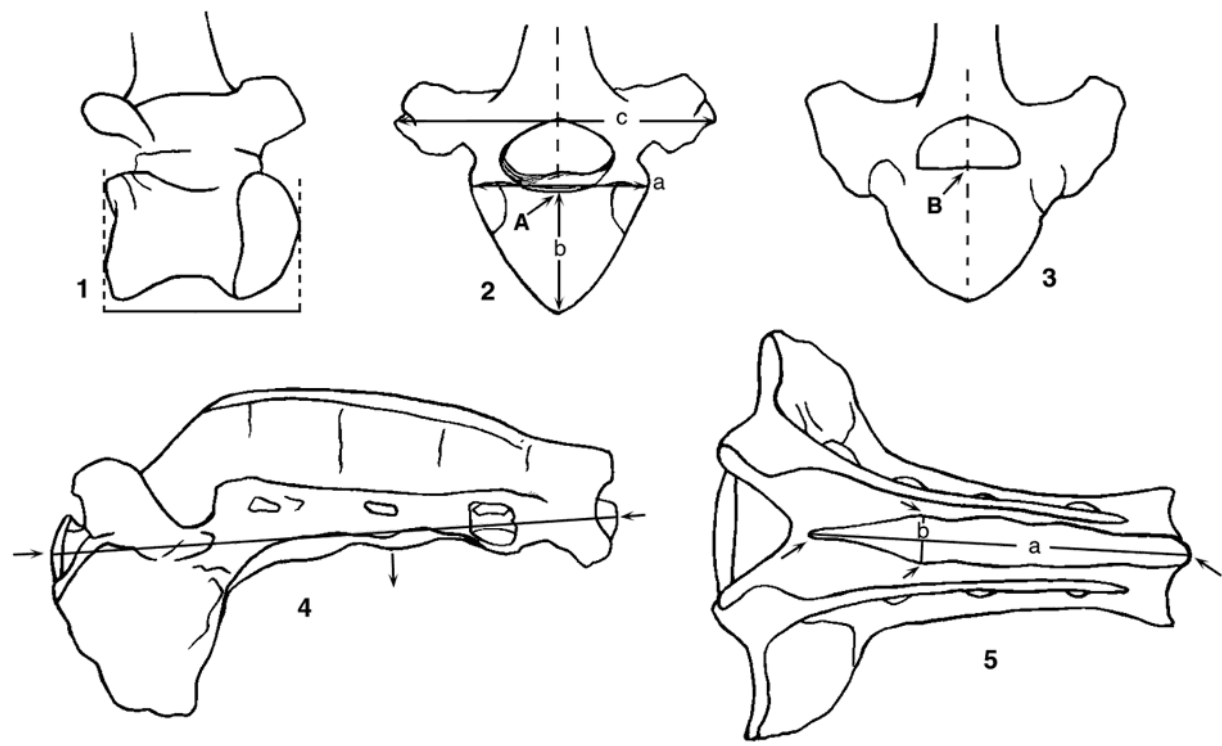

1, 2 et 3 - Vertèbre thoracique ; 1 - vue latérale ; 2 - vue crâniale ; 3 - vue caudale, 4 et 5 - Sacrum, 4 vue latérale ; 5 - vue dorsale. 
Figure 23 : Bovinés. Vertèbres cervicales ; emplacement des mesures effectuées.

Figure 23: Bovine cervical vertebrae, showing the location of measurements reported in the text.
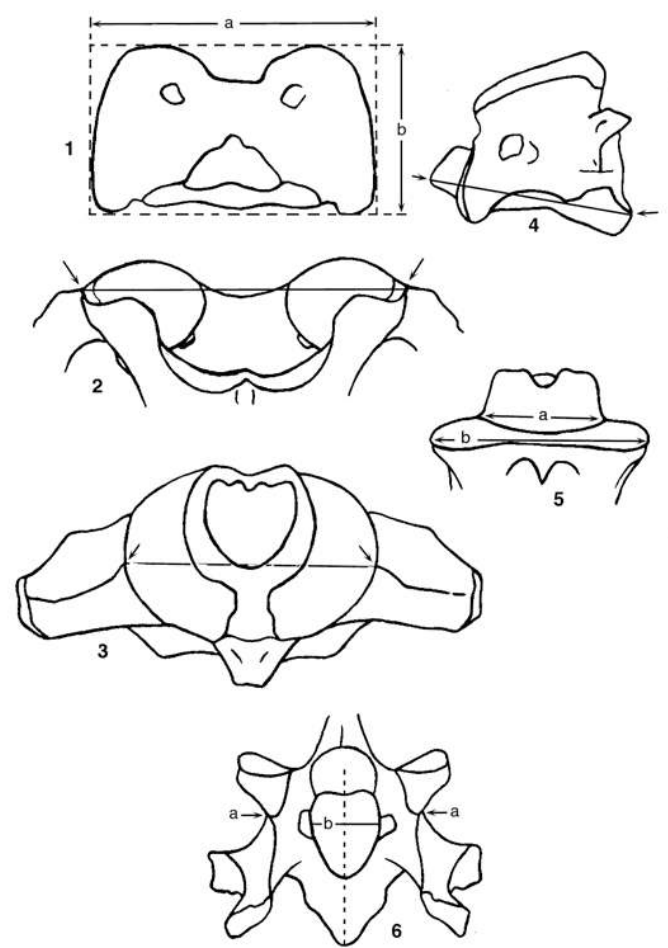

1, 2 et 3 - Atlas ; 1 - vue dorsale ; 2 - région articulaire antérieure ; 3 - vue caudale 4 et 5 - Axis ; 4 - vue latérale ; 5 - région articulaire antérieure

6 - Autre vertèbre cervicale; vue crâniale 
Figure 24 - Habarra. Secteur E. Plan de répartition des ossements. Figure 24 - The distribution of bones in Habarra Cave, Sector $E$.

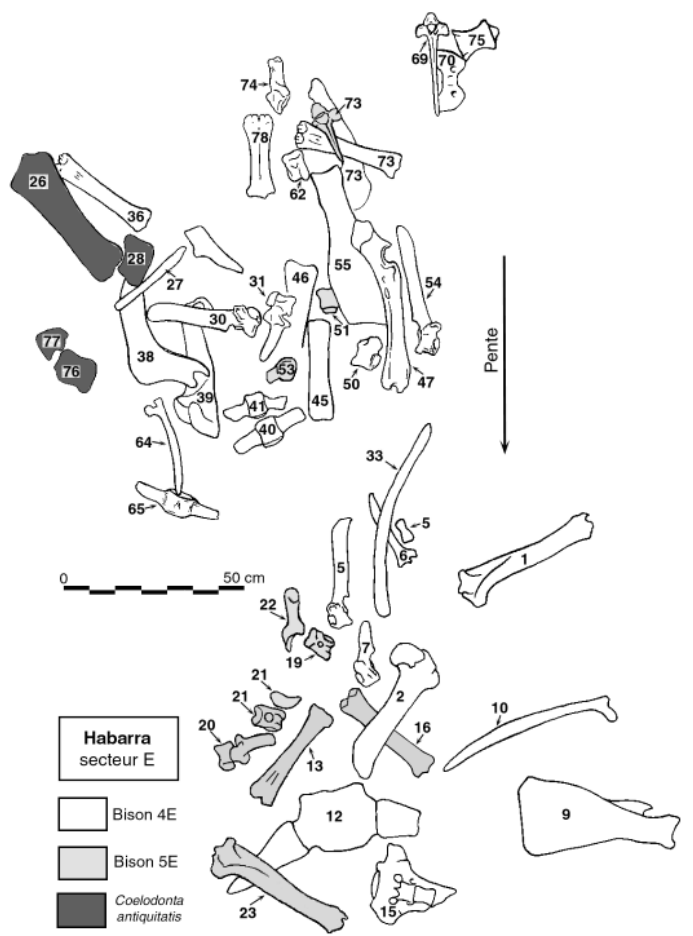

En blanc : bison femelle 4E; i en gris clair : bison femelle $5 \mathrm{E}$; en gris foncé : Coelodonta antiquitatis 
Figure 25 - Bison femelle 4E d'Habarra. Processus cornual gauche E12, sections. Figure 25 - Bison female 4E from Habarra Cave: Cross-sections of left horn core E12.

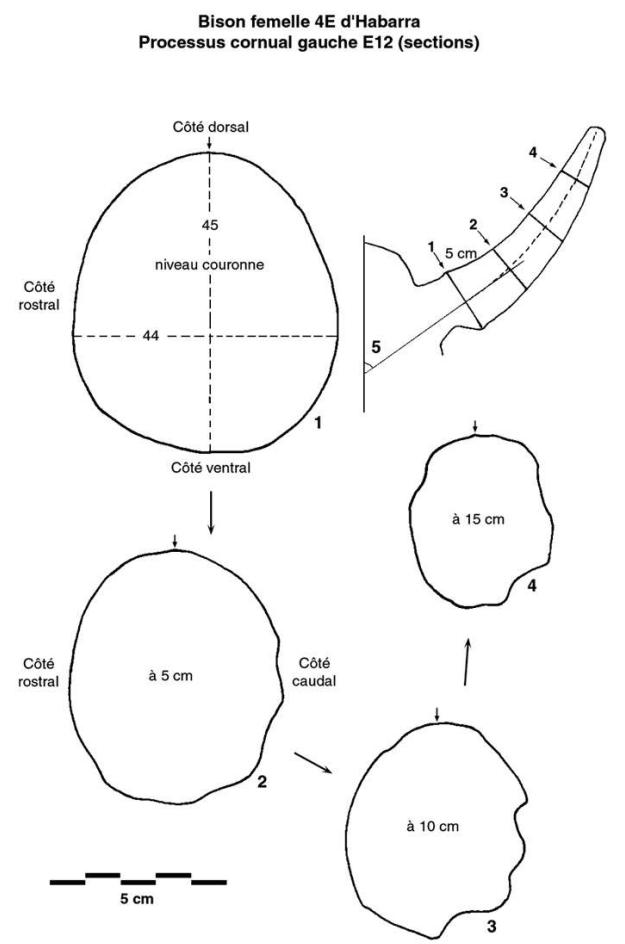

5 : angle caudal réalisé par la région proximale du processus cornual avec le plan sagittal

Figure 26 - Bison femelle 6F d'Habarra. Processus cornual gauche F10, sections. Figure 26 - Bison female 6F from Habarra Cave: Cross-sections of left horn core F10.

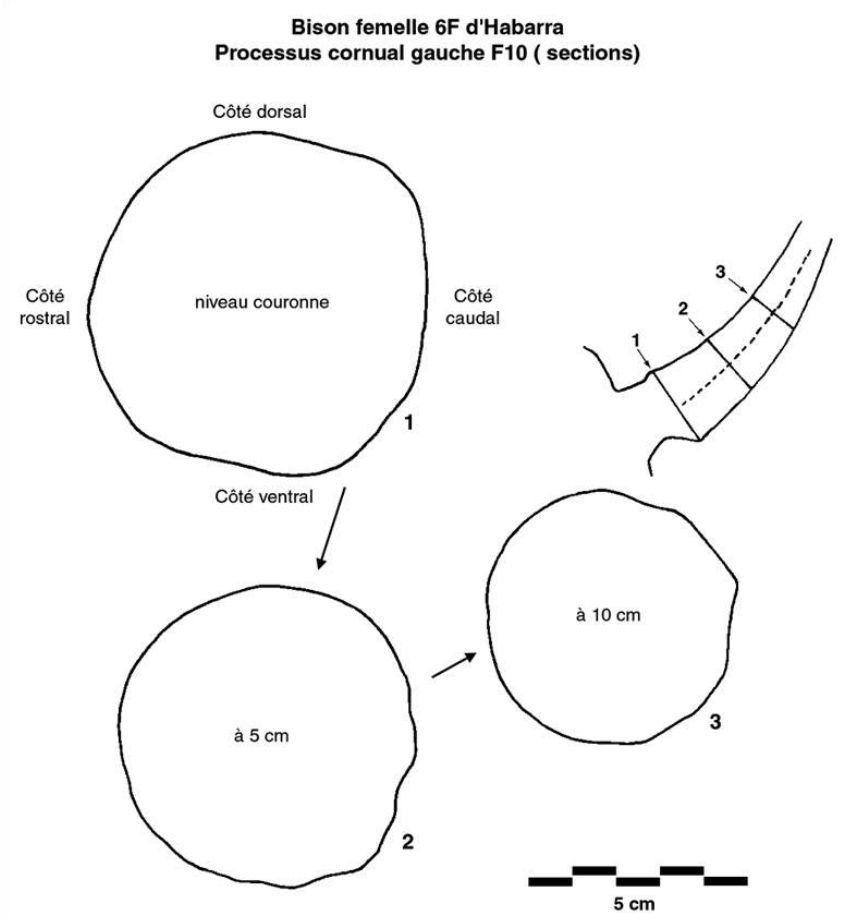




\section{Tableaux 1 à 42}

Tableau 1 - Genre Bison. Bisons femelles. Os longs, dimensions et indices. Table 1 - Long bone dimensions and index values for female Bison.

\begin{tabular}{|c|c|c|c|c|c|c|c|c|c|c|c|c|c|}
\hline & \multicolumn{5}{|c|}{ Bison bonasuss } & & \multicolumn{3}{|c|}{ Bison priscus d'Habarra } \\
\hline & $\begin{array}{c}\text { B.M. } \\
1892 \\
3.15 \\
2\end{array}$ & $\begin{array}{c}\text { B.M. } \\
1947 \\
7.16 \\
5\end{array}$ & $\begin{array}{c}\text { B.M. } \\
1949 \\
6-29 \\
1\end{array}$ & $\begin{array}{l}\text { M.P. } \\
1971 \\
144\end{array}$ & & $\begin{array}{c}\text { B.M. } \\
1850 \\
11.22 \\
102 \\
\end{array}$ & $\begin{array}{l}\text { B.M. } \\
1865 \\
12-8 \\
26 \\
\end{array}$ & $\begin{array}{l}\text { M.P. } \\
1898 \\
261\end{array}$ & $\begin{array}{c}\text { I.P.G.Q. } \\
C \\
5313\end{array}$ & $\begin{array}{c}\text { D'après } \\
\text { McDonald, 1981 } \\
\text { tabl. 30, p. } 97\end{array}$ & $\mathrm{AAB}$ & $4 \mathrm{E}$ & $5 \mathrm{E}$ \\
\hline $\begin{array}{l}\text { (1) - Humérus } \\
\text { Longueur }\end{array}$ & 2910 & 326.0 & 3220 & 323,0 & & 297,0 & 2710 & 282.0 & 283.5 & & 328 & 333,5 & \\
\hline $\begin{array}{l}\text { (2)- Humérus } \\
\text { largeur minimum } \\
\text { 100/Longueur }\end{array}$ & 17,8 & 16,8 & 16,7 & 000,0 & & 17,1 & 16,9 & 202,0 & 14,1 & & 16,1 & 14,9 & \\
\hline $\begin{array}{l}\text { (3) - Radius } \\
\text { Longueur }\end{array}$ & 304,0 & 340,0 & 322,0 & 343,5 & $\begin{array}{l}\text { (Millot, 1945) } \\
335,0 ; 340,0\end{array}$ & 311,0 & 2990 & 2980. & 2940 & & 3330 & 355,5 & \\
\hline \begin{tabular}{|c|} 
(4) - Radius \\
largeur minimum * \\
100/Longueur
\end{tabular} & 14,1 & 12,8 & 13,6 & & & 13,5 & 13,5 & & 14,3 & & 15,6 & 14,4 & \\
\hline $\begin{array}{l}\text { (5)-Métacarpe } \\
\text { Longueur }\end{array}$ & 208,0 & & 208,0 & 216,0 & $\begin{array}{c}\text { (Reshetov, 1974) } \\
\text { IV. 202-209 } \\
\text { moy. }=205 ; \mathrm{n}=11\end{array}$ & 209,0 & 201,0 & 193,5 & 198,0 & $\begin{array}{l}\text { I.V. : } 182-219 ; n=164 \\
\text { I.C. moy. }=198,9 \pm 0,5\end{array}$ & 220,0 & 232,0 & \\
\hline 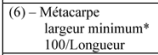 & 17,5 & & 17,8 & 18,5 & $\begin{array}{l}\text { d'apress moyennes } \\
\text { (Reshetov, } 1994 \text { ) } \\
\text { moy. }=17,2\end{array}$ & 16,3 & 17,9 & 17,6 & 18,7 & $\begin{array}{c}\text { d'après moyennes } \\
18,4\end{array}$ & 22,2 & 17,8 & \\
\hline \begin{tabular}{|l}
$(7)-(5) * * 00 /(1)$ \\
\end{tabular} & 71,4 & & 64,5 & 66,8 & & 70,3 & 74,1 & 68,6 & 69,8 & & 67,0 & 69,5 & \\
\hline$(8)-(5)^{*}+100 /(3)$ & 68,4 & & 64,5 & 62,8 & & 67,2 & 67,2 & 64,9 & 67,3 & & 66,0 & 65,2 & \\
\hline$(9)-(5) * 100 /(14)$ & 83,2 & & 81,2 & & & 81,6 & 81,7 & 80,7 & 82,1 & d'après moyennes 81,2 & 83,3 & 81,4 & \\
\hline $\begin{array}{l}\text { (10) }- \text { Fémur } \\
\text { Longueur }\end{array}$ & 382.0 & 414,0 & 394,0 & 417,5 & & 361,0 & 352.0 & 351,0 & 365.5 & & & 427,0 & \\
\hline \begin{tabular}{|c|} 
(11) - F́́mur \\
largeur minimum * \\
100/Longueur
\end{tabular} & 10,2 & 10,4 & 10,1 & & & 10,5 & 10,2 & & 10,7 & & & 10,6 & \\
\hline \begin{tabular}{|l|} 
(12) - Tibia \\
Longueur
\end{tabular} & 402,0 & 414,0 & 425,0 & 439,0 & $\begin{array}{l}\text { (Millot, 1945) } \\
430 ; 445\end{array}$ & 376,0 & 360,0 & 360,0 & & & 402,0 & 436,0 & 420,6 \\
\hline $\begin{array}{l}\text { (13) - Tibia } \\
\text { largeur minimum * } \\
\text { 100/Longueur }\end{array}$ & 10,9 & 10,1 & 10,3 & & & 10,4 & 11,7 & & & & 12,6 & 11,2 & 11,9 \\
\hline \begin{tabular}{|c|} 
(14) - Metatararse \\
Longueur
\end{tabular} & 250,0 & & 256,0 & 273,0 & $\begin{array}{l}\text { (Millot, 1945) } \\
271 ; 265\end{array}$ & 256,0 & 246,0 & 239.5 & 241,0 & $\begin{array}{l}\text { I.V. : } 224-264 ; n=177 \\
\text { I.C. moy }=245,2 \pm 0,6\end{array}$ & 264,0 & 285,0 & 278,5 \\
\hline \begin{tabular}{|c|} 
(15) Métatarse \\
largeur minimum * * \\
100/Longueur
\end{tabular} & 12,8 & & 11,7 & 13,2 & & 11,1 & 11,8 & & 11,4 & $\begin{array}{c}\text { d'après moyennes } \\
11,9\end{array}$ & 15,1 & 12,6 & 13,2 \\
\hline$(16)-(14) * 100 /(10)$ & 65,4 & & 64,9 & 65,3 & & 70,9 & 69,8 & 68,2 & 65,9 & & & 66,7 & \\
\hline$(17)-(14) * 100 /(12)$ & 02,1 & & 60,2 & $62, \mathrm{I}$ & $\begin{array}{l}\text { (Millot, 1945) } \\
63,02 ; 59,55\end{array}$ & \begin{tabular}{|l|l}
68,0 \\
\end{tabular} & 68,3 & 66,5 & & & 65,4 & 65, &, 3 \\
\hline
\end{tabular}

Toutes les mesures sont en millimètres; I.V. $=$ intervalle de variation; moy $=$ moyenne $;$ I.C. moy. $=$ intervalle de confiance de la moyenne; $n=$ nombre de spécimen B.M. = British Museum (les mesures y ont eté prises par Norah Byrne); M.P. = Mure
Institut de Préhistoire et de Géologie du Quaternaire de l'Université Bordeaux I.

Tableau 2 - Genre Bison. Bisons mâles. Os longs, dimensions et indices.

Table 2 - Long bone dimensions and index values for male Bison.

\begin{tabular}{|c|c|c|c|c|c|c|c|c|c|c|}
\hline & \multicolumn{5}{|c|}{ Bison bonasus } & \multicolumn{4}{|c|}{ Bison bison } & \multirow{3}{*}{$\begin{array}{c}\text { Bison priscus Habarra } \\
2 \mathrm{AB}\end{array}$} \\
\hline & $\begin{array}{l}\text { B.M. } \\
1892 \\
315\end{array}$ & B.M. & $\begin{array}{l}\text { B.M. } \\
1845\end{array}$ & \begin{tabular}{|l|} 
M.P. \\
1978 \\
\end{tabular} & & $\begin{array}{l}\text { B.M. } \\
851 \mathrm{a}\end{array}$ & $\begin{array}{l}\text { B.M. } \\
1953\end{array}$ & I.P.G.Q. & $\begin{array}{c}\text { D'après } \\
\text { McDonald, } 1981\end{array}$ & \\
\hline & & & & & & & & & tabl. 30,p. 97 & \\
\hline Longueur & 327,0 & 333,0 & 352,0 & 359,0 & & 328,0 & 335,0 & 323,0 & & $372 \mathrm{env}$ \\
\hline $\begin{array}{l}\text { (2)- Humérus } \\
\text { largeur minimum } \\
100 / \text { Longueur } \\
\end{array}$ & 19,1 & 14,4 & 18,4 & & & 18,9 & 21,1 & 17,2 & & $16,9 ?$ \\
\hline $\begin{array}{l}\text { (3)- } \text { Radius } \\
\text { Longueur }\end{array}$ & 327,0 & 342,0 & 349,0 & 365,0 & $\begin{array}{c}\text { (Millo, 1945) } \\
\text { I.V.= }=336,0-371,0 \\
\text { moy. }=354,3 ; \mathrm{n}=6\end{array}$ & 344,0 & 339,0 & 351,5 & & 378,0 \\
\hline \begin{tabular}{|l|} 
(4) - Radius \\
largeur minimum * \\
100 /Longueur \\
\end{tabular} & 14,8 & 12,9 & 15,2 & & & 13,4 & 16,2 & 17,4 & & 16,9 \\
\hline $\begin{array}{l}\text { (5)-Métacarpe } \\
\text { Longueur }\end{array}$ & 202,0 & & 205,0 & 227,5 & $\begin{array}{l}\text { (Reshetov, 1974) } \\
I .,=202,0-225,0 \\
\text { moy. }=215 ; \mathrm{n}=26\end{array}$ & 203,0 & 212,0 & 201,5 & $\begin{array}{l}\text { I.V. }=192-227 ; n=112 \\
\text { I.C. moy. }=206,3 \pm 0,6\end{array}$ & 229 \\
\hline \begin{tabular}{|c|} 
(6) - Métacarpe \\
largeur minimum \\
100/Longueur
\end{tabular} & 21,5 & & 22,9 & 19,8 & $\begin{array}{l}\text { d'après moyennes } \\
\text { (Reshetov, 1974) } \\
\text { moy. = 20,5 }\end{array}$ & 21,7 & 22,6 & 25,5 & $\begin{array}{l}\text { d'après moyennes } \\
\begin{array}{c}45,4 * 100 / 206,3 \\
=\end{array} \\
\end{array}$ & 25,1 \\
\hline$\frac{(7)-(5) * 100 /(1)}{(8)-(5) * 100 /(3)}$ & $\begin{array}{l}61,7 \\
61,7 \\
\end{array}$ & & $\begin{array}{l}58,2 \\
58,7\end{array}$ & \begin{tabular}{|l|}
63,3 \\
62,3 \\
\end{tabular} & & $\begin{array}{l}61,8 \\
59,0\end{array}$ & $\begin{array}{l}63,2 \\
62,5 \\
\end{array}$ & $\begin{array}{l}62,3 \\
60,7\end{array}$ & & $\frac{61,5 ?}{60,5}$ \\
\hline$(9)-(5) * 100 /(14)$ & 81,4 & & 79,1 & 81,8 & & 82,1 & 80,0 & & & 78,9 \\
\hline $\begin{array}{c}\text { (10) - Fémur } \\
\text { Longueur }\end{array}$ & 421,0 & 421,0 & 444,0 & 462.0 & & 406.0 & 404,0 & 410.0 & & 473 \\
\hline $\begin{array}{l}\text { (11)- Fémur } \\
\text { largeur minimum * } \\
\text { 100/Longueur }\end{array}$ & 10,7 & 9,5 & 10,6 & & & 10,8 & 11,5 & 11,8 & & 11,5 \\
\hline $\begin{array}{l}\text { (12) - Tibia } \\
\text { Longueur }\end{array}$ & 427,0 & 445,0 & 455,0 & 472,5 & $\begin{array}{c}\text { (Millot, 1945) } \\
\text { I.V. }=436,0-478,0 \\
\text { moy }=464,3 ; \mathrm{n}=6\end{array}$ & 404,0 & 415,0 & 412,0 & & 476,0 \\
\hline $\begin{array}{l}\text { (13) - Tibia } \\
\text { largeur minimum * } \\
\text { 100/Longueur }\end{array}$ & 11,9 & & 9,5 & $11,6 ?$ & & 11,4 & 12,3 & 13,1 & & 13,0 \\
\hline $\begin{array}{l}\text { (14) - Métatarse } \\
\text { Longucur }\end{array}$ & 248,0 & & 259,0 & 278,0 & $\begin{array}{c}\text { (Millot, 1945) } \\
\text { I.V.= }=249,0-275,0 \\
\text { moy }=264,0: n=4 \\
\end{array}$ & 252,0 & 265,0 & & $\begin{array}{l}\text { I.V. }=232-276 ; n=118 \\
\text { I.C. moy. }=255,2 \pm 0,7\end{array}$ & 290,0 \\
\hline $\begin{array}{l}\text { (15) Métatarse } \\
\text { largeur minimum * } \\
100 / \text { Longueur } \\
\end{array}$ & 15,1 & & 15,8 & 13,7 & & 13,1 & 13,4 & & $\begin{array}{l}\text { daprès moyennes } \\
35,3 * 100 / 255,2 \\
=13,8\end{array}$ & 15,5 \\
\hline \begin{tabular}{|l|}
$(16)-(14)^{*}$ \\
$1000 /(10)$ \\
\end{tabular} & 58,9 & & 58,3 & 60,1 & & 62,0 & 65,5 & & & 61,3 \\
\hline$(17)-(14) * 100 /(12)$ & 58,0 & & 56,9 & 58,8 & & 62,3 & 63,8 & & & 60,9 \\
\hline
\end{tabular}


Tableau 3 - Bison priscus. Crâne, dimensions et indices. Table 3 - Bison priscus skull dimensions and index values.

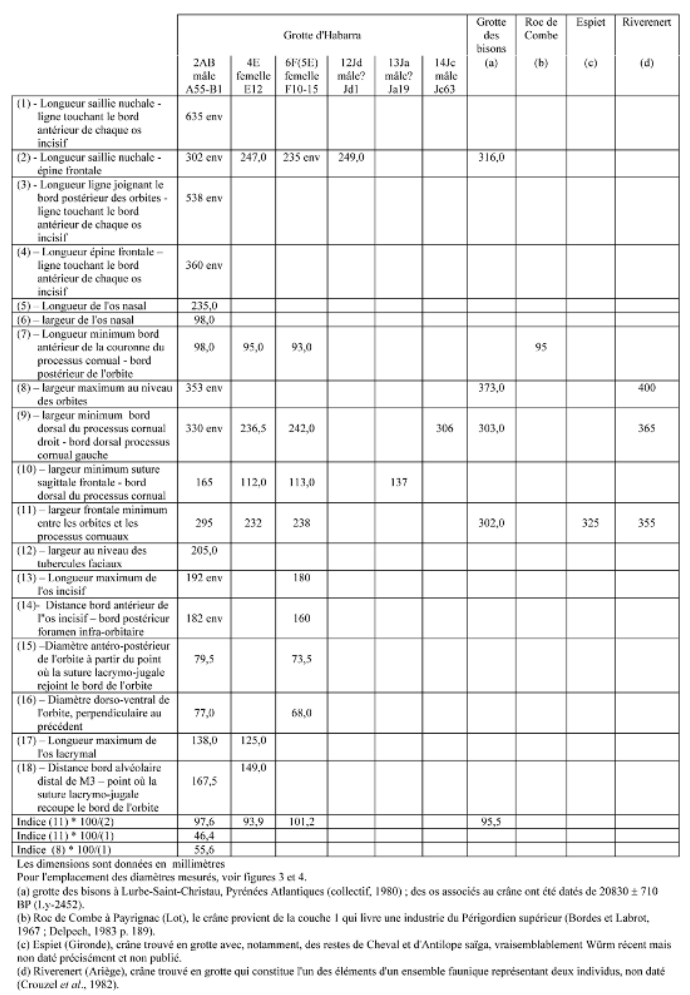

Tableau 4 - Bison priscus. Crâne, dimensions. Table 4 - Bison priscus skull dimensions.

\begin{tabular}{|c|c|c|c|c|c|c|c|}
\hline & \multicolumn{4}{|c|}{ Grotte d'Habarra } & \multirow[b]{2}{*}{$\begin{array}{c}\text { Grotte } \\
\text { des } \\
\text { bisons } \\
\text { (a) }\end{array}$} & \multirow[b]{2}{*}{$\begin{array}{l}\text { Roc de } \\
\text { Combe } \\
\text { (b) }\end{array}$} & \multirow[b]{2}{*}{$\begin{array}{c}\text { Espiet } \\
\text { (c) }\end{array}$} \\
\hline & $\begin{array}{c}2 \mathrm{AB} \\
\text { mâle } \\
\mathrm{A} 55-\mathrm{B} 1\end{array}$ & $\begin{array}{c}4 \mathrm{E} \\
\text { femelle } \\
\mathrm{E} 12\end{array}$ & $\begin{array}{c}6 \mathrm{~F}(5 \mathrm{E}) \\
\text { femelle } \\
\text { F10-15 }\end{array}$ & $\begin{array}{c}12 \mathrm{Jd} \\
\text { mâle? } \\
\mathrm{Jdl} \text { ? }\end{array}$ & & & \\
\hline $\begin{array}{l}\text { (19) - largeur maximum au niveau des } \\
\text { processus mastö̈des } \\
\end{array}$ & $297 ?$ & 227,0 & & 231,5 & & & 265 \\
\hline $\begin{array}{l}\text { (20) - largeur maximum de l'occipital au } \\
\text { niveau des processus jugulaires }\end{array}$ & 237,0 & 187,0 & & 187,5 & & & \\
\hline $\begin{array}{l}\text { (21) - largeur minimum entre les deux } \\
\text { lignes temporales }\end{array}$ & & 166 & 147 & & & 185 & 201 \\
\hline $\begin{array}{l}\text { (22) - largeur maximum du foramen } \\
\text { magnum }\end{array}$ & 36,0 & 49,0 & & 44,5 & & 52 & \\
\hline (23) - hauteur du foramen magnum & 49,0 & 45,0 & & 42,0 & & & \\
\hline (24) - largeur bicondylienne & 139,5 & 127,0 & & 129,0 & & 150,0 & 144,0 \\
\hline $\begin{array}{l}\text { (25) - distance minimum du bord } \\
\text { supérieur du foramen magnum à la } \\
\text { saillie nuchale }\end{array}$ & 113 env & 86,5 & & 97,5 & 121 & & \\
\hline \begin{tabular}{|l|} 
(26) - Longueur condylo-basale \\
\end{tabular} & $597 \mathrm{env}$ & & & & & & \\
\hline (27) - Longueur basale & $556 \mathrm{env}$ & & & & & & \\
\hline $\begin{array}{l}\text { (28) - Longueur de la partie basilaire de } \\
\text { l'occipital }\end{array}$ & 133 & 115 & & 107,5 & & 135 & \\
\hline $\begin{array}{l}\text { (29) - largeur maximum du basioccipital } \\
\text { au niveau des revers basilaires des } \\
\text { condyles }\end{array}$ & 88,5 & 67,0 & & 66,5 & & & \\
\hline $\begin{array}{l}\text { (30) - largeur minimum de la partie } \\
\text { basilaire des condyles }\end{array}$ & 70,5 & 59,0 & & 64,5 & & & 76,0 \\
\hline $\begin{array}{l}\text { (31) - largeur du palais au niveau du } \\
\text { bord alvéolaire distal des M3 }\end{array}$ & & 88,5 & & & & & \\
\hline $\begin{array}{l}\text { (32) - Longueur de la série P2-M3 : du } \\
\text { bord alvéolaire mésial de P2 au bord } \\
\text { alvéolaire distal de M3 }\end{array}$ & 160,0 & & 156,5 & & & & \\
\hline $\begin{array}{l}\text { (33) - Longueur de la série P2-P4 } \\
\text { mesurée du côté vestibulaire }\end{array}$ & 62,0 & & 62,5 & & & & \\
\hline $\begin{array}{l}\text { (34) - Longueur de la série P4-M3 } \\
\text { mesurée du côté vestibulaire }\end{array}$ & 104 & & 94,5 & & 106,5 & & \\
\hline $\begin{array}{l}\text { Les dimensions sont données en millimètrte } \\
\text { Pour l'emplacement des diamètres mesures } \\
\text { (a) grotte des bisons à Lurbe-Saint-Christar } \\
\text { été datés de } 20830 \pm 710 \text { BP (Ly-2452). } \\
\text { (b) Roc de Combe à Payrignac (Lot), le cra } \\
\text { supérieur (Bordes et Labrot, 1967; Delpect } \\
\text { (c) Espiet (Gironde), crâne trouvé en grotte } \\
\text { vraisemblablement Würm récent mais non }\end{array}$ & $\mathrm{ec}, \mathrm{n}$ & A & 1 & & 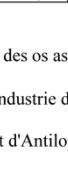 & ciés & $\begin{array}{l}\text { râne ont } \\
\text { ien }\end{array}$ \\
\hline
\end{tabular}


Tableau 5 - Bison priscus. Crâne, processus cornuaux ; dimensions. Table 5 - Bison priscus horn core dimensions.

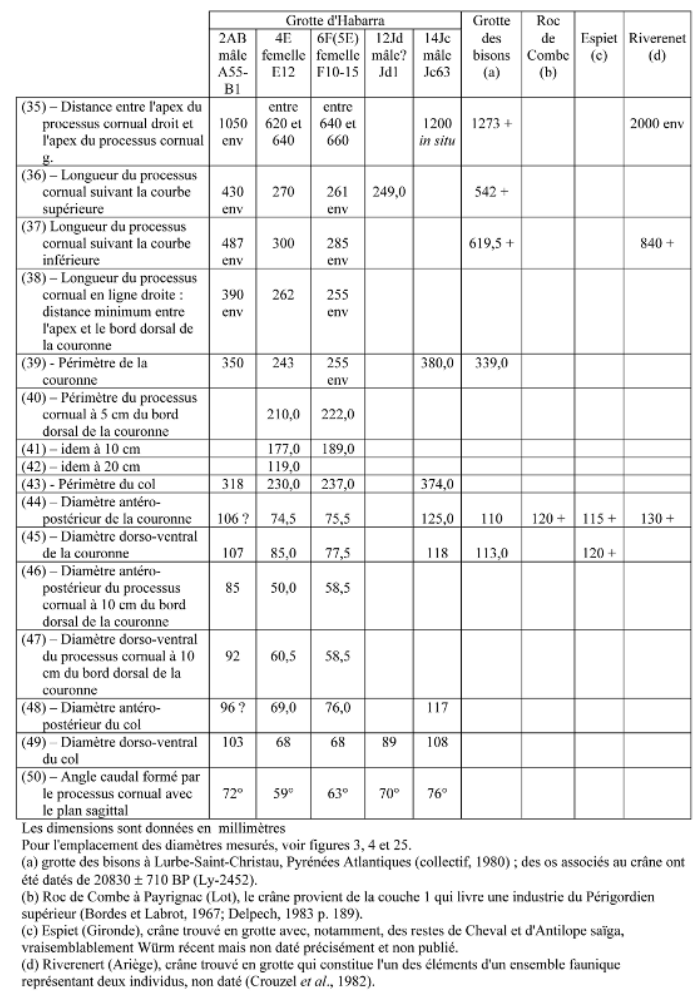

Tableau 6 - Bison priscus. Crâne, dimensions et indices. Table 6 - Bison priscus skull dimensions and index values.

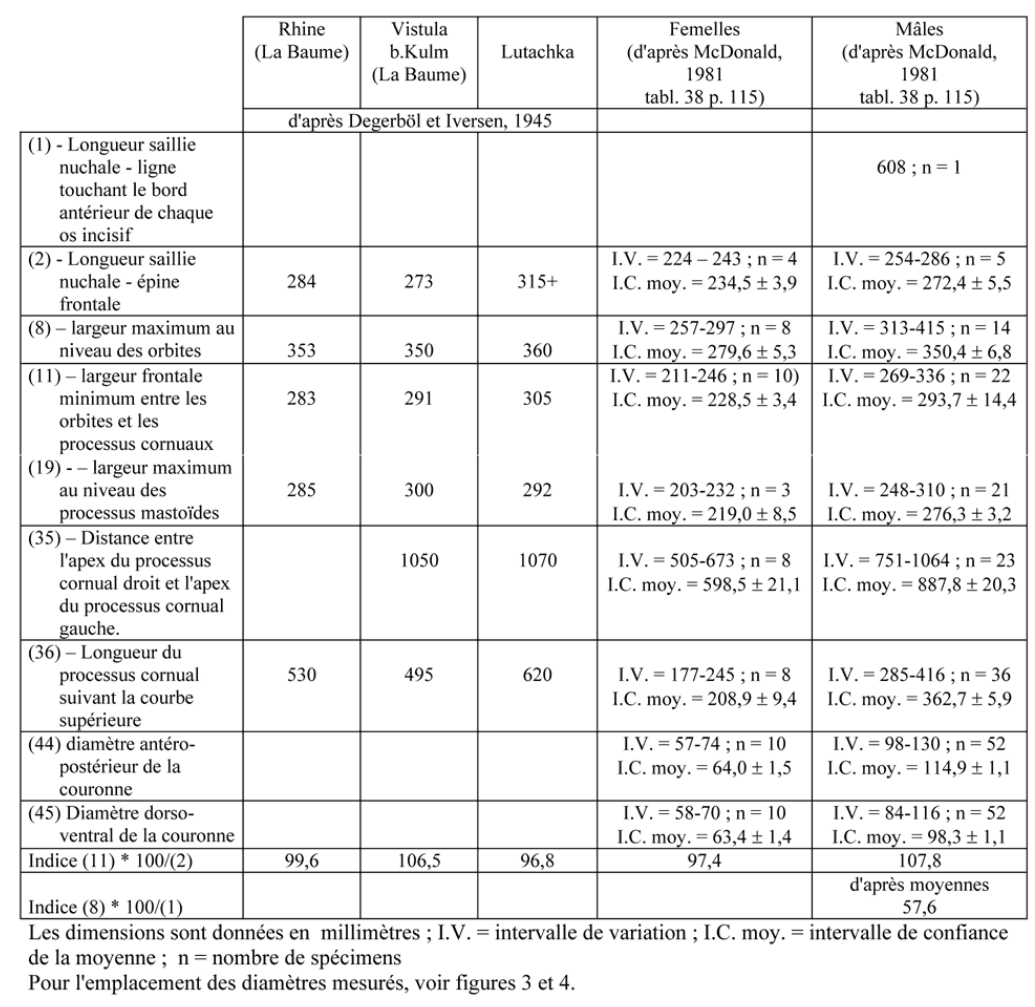


Tableau 7 - Bison bonasus femelles. Crâne, dimensions et indices. Table 7 - Bison bonasus female skull dimensions and index values.

\begin{tabular}{|c|c|c|c|c|c|c|}
\hline & $\begin{array}{l}\text { B.M. } \\
\text { 1949-6- } \\
29-1\end{array}$ & $\begin{array}{l}\text { B.M. } \\
1892-3- \\
15-2\end{array}$ & $\begin{array}{l}\text { B.M. } \\
1947-7- \\
16-5\end{array}$ & $\begin{array}{l}\text { M.P. } \\
1971- \\
144\end{array}$ & $\begin{array}{l}\text { Lithuanie (La } \\
\text { Baume) } \\
\text { in Degerböl et } \\
\text { Iversen (1945) }\end{array}$ & $\begin{array}{l}\text { d'après Allen } \\
\text { in Degerböl et } \\
\text { Iversen (1945) }\end{array}$ \\
\hline $\begin{array}{l}\text { (1) - Longueur saillie nuchale - } \\
\text { ligne touchant le bord antérieur } \\
\text { de chaque os incisif }\end{array}$ & 490 & 483 & 515 & 506 & & \\
\hline $\begin{array}{l}\text { (2) - Longueur saillie nuchale - } \\
\text { épine frontale }\end{array}$ & & & & 227 & 234 & 208 \\
\hline $\begin{array}{l}\text { (8) - largeur maximum au niveau } \\
\text { des orbites }\end{array}$ & 249 & 247 & 276 & 273 & 278 & 230 \\
\hline $\begin{array}{l}\text { (9) - largeur minimum bord dorsal } \\
\text { du processus cornual droit - } \\
\text { bord dorsal processus cornual } \\
\text { gauche }\end{array}$ & 205 & 214 & 207 & 225 & 226 & \\
\hline $\begin{array}{l}\text { (11) - largeur frontale minimum } \\
\text { entre les orbites et les } \\
\text { processus cornuaux }\end{array}$ & 194 & 203 & 218 & 220 & 217 & 206 \\
\hline $\begin{array}{l}\text { (35) - Distance entre l'apex du } \\
\text { processus cornual droit et } \\
\text { l'apex du processus cornual } \\
\text { gauche }\end{array}$ & 402 & 389 & & 431 & 440 & \\
\hline $\begin{array}{l}\text { (36) - Longueur du processus } \\
\text { cornual suivant la courbe } \\
\text { supérieure }\end{array}$ & 108 & 128 & & 153 & & 153 \\
\hline Indice $(11) * 100 /(2)$ & & & & 96,9 & 92,7 & 99,0 \\
\hline Indice $(11) * 100 /(1)$ & 39,5 & 42,0 & 42,3 & 43,4 & & \\
\hline Indice $(8) * 100 /(1)$ & 50,8 & 51,1 & 53,6 & 54,0 & & \\
\hline
\end{tabular}

Les dimensions sont données en millimètres

Pour l'emplacement des diamètres mesurés, voir figures 3 et 4

B.M. = British Museum ( les mesures y ont été prises par Norah Byrne)

M.P. = Muséum National d'Histoire Naturelle, Laboratoire d'Anatomie Comparée.

Tableau 8 - Bison bonasus mâles. Crâne, dimensions et indices. Table 8 - Bison bonasus male skull dimensions and index values.

\begin{tabular}{|c|c|c|c|c|c|c|c|c|}
\hline \multirow[b]{2}{*}{$\begin{array}{l}\text { (1) - Longueur saillie nuchale - ligne } \\
\text { touchant le bord antérieur de } \\
\text { chaque os incisif }\end{array}$} & \multirow{2}{*}{$\begin{array}{c}\text { B.M. } \\
1892-3- \\
15-1 \\
\\
518\end{array}$} & \multirow{2}{*}{$\begin{array}{c}\text { B.M. } \\
1845- \\
10- \\
13-2 \\
\\
524\end{array}$} & \multirow{2}{*}{$\begin{array}{l}\text { M.P. } \\
510\end{array}$} & \multirow{2}{*}{$\begin{array}{c}\text { Lithuanie } \\
\text { (La Baume) } \\
\text { in Degerböl et } \\
\text { Iversen (1945) }\end{array}$} & \multicolumn{4}{|c|}{$\begin{array}{c}\text { d'après Allen } \\
\text { in Degerböl et Iversen (1945) }\end{array}$} \\
\hline & & & & & & & & \\
\hline $\begin{array}{l}\text { (2) - Longueur saillie nuchale - } \\
\text { épine frontale }\end{array}$ & & & 245 & 275 & 285 & 257 & 250 & 265 \\
\hline $\begin{array}{l}\text { (8) - largeur maximum au niveau } \\
\text { des orbites }\end{array}$ & 295 & 326 & 285 & 335 & 310 & 328 & 310 & 312 \\
\hline $\begin{array}{l}\text { (9) - largeur minimum bord dorsal } \\
\text { du processus cornual droit - bord } \\
\text { dorsal processus cornual gauche }\end{array}$ & 260 & 223 & 246 & 296 & & & & \\
\hline $\begin{array}{l}\text { (11) - largeur frontale minimum } \\
\text { entre les orbites et les processus } \\
\text { cornuaux }\end{array}$ & 248 & 246 & 231 & 271 & 270 & 245 & 231 & 267 \\
\hline $\begin{array}{l}\text { (35) - Distance entre l'apex du } \\
\text { processus cornual droit et l'apex } \\
\text { du processus cornual gauche }\end{array}$ & 616 & 612 & & 635 & & & & \\
\hline $\begin{array}{l}\text { (36) - Longueur du processus } \\
\text { cornual suivant la courbe } \\
\text { supérieure }\end{array}$ & 240 & 237 & & 225 & 190 & 230 & & 270 \\
\hline Indice $(11) * 100 /(2)$ & & & 94,2 & 98,5 & 94,7 & 95,3 & 92,4 & 100,7 \\
\hline Indice $(11) * 100 /(1)$ & 47,8 & 46,9 & 45,2 & & & & & \\
\hline Indice $(8) * 100 /(1)$ & 56,9 & 62,2 & 55,9 & & & & & \\
\hline
\end{tabular}

Les dimensions sont données en millimètres

Pour l'emplacement des diamètres mesurés, voir figures 3 et 4

B.M. = British Museum ( les mesures y ont été prises par Norah Byrne)

M.P. = Muséum National d'Histoire Naturelle, Laboratoire d'Anatomie Comparée 
Tableau 9 - Bison bison femelles. Crâne, dimensions et indices. Table 9 - Bison bison female skull dimensions and index values.

\begin{tabular}{|c|c|c|c|}
\hline & $\begin{array}{c}\text { B.M. } \\
1850-11- \\
22-102\end{array}$ & $\begin{array}{c}\text { M.P. } \\
1854- \\
154 \mathrm{n}^{\circ} 8\end{array}$ & $\begin{array}{c}\text { Bison bison bison } \\
\text { in McDonald } \\
(1981, \text { tabl. 29, p. } 96)\end{array}$ \\
\hline $\begin{array}{l}\text { (1) - Longueur saillie nuchale - ligne } \\
\text { touchant le bord antérieur de } \\
\text { chaque os incisif }\end{array}$ & 488 & 513 & $\begin{array}{l}\text { I.V. }=464-516 ; n=19 \\
\text { I.C. moy. }=484,7 \pm 3,2\end{array}$ \\
\hline $\begin{array}{l}\text { (2) - Longueur saillie nuchale - épine } \\
\text { frontale }\end{array}$ & & 227 & $\begin{array}{l}\text { I.V. }=195-228 ; n=24 \\
\text { I.C. moy. }=212,4 \pm 1,6\end{array}$ \\
\hline $\begin{array}{l}\text { (8) - largeur maximum au niveau } \\
\text { des orbites }\end{array}$ & 256 & 272 & $\begin{array}{l}\text { I.V. }=248-291 ; n=25 \\
\text { I.C. moy. }=267,5 \pm 2,0\end{array}$ \\
\hline $\begin{array}{l}\text { (9) - largeur minimum bord dorsal } \\
\text { du processus cornual droit - bord } \\
\text { dorsal processus cornual gauche }\end{array}$ & 207 & 249 & \\
\hline $\begin{array}{l}\text { (11) - largeur frontale minimum } \\
\text { entre les orbites et les processus } \\
\text { cornuaux }\end{array}$ & 205 & 215 & $\begin{array}{l}\text { I.V. }=198-233 ; \mathrm{n}=27 \\
\text { I.C. moy. }=216,7 \pm 1,7\end{array}$ \\
\hline $\begin{array}{l}\text { (35) - Distance entre l'apex du } \\
\text { processus cornual droit et l'apex } \\
\text { du processus cornual gauche }\end{array}$ & $475+$ & & $\begin{array}{l}\text { I.V. }=397-514 ; \mathrm{n}=28 \\
\text { I.C. moy. }=451,0 \pm 6,6\end{array}$ \\
\hline $\begin{array}{l}\text { (36) - Longueur du processus } \\
\text { cornual suivant la courbe } \\
\text { supérieure }\end{array}$ & 128 & & $\begin{array}{l}\text { I.V. }=93-177 ; \mathrm{n}=34 \\
\text { I.C. moy. }=124,1 \pm 3,0\end{array}$ \\
\hline $\begin{array}{l}\text { (44) - diamètre antéro-postérieur de } \\
\text { la couronne du processus cornual }\end{array}$ & & & $\begin{array}{l}\text { I.V. }=44-612 ; n=38 \\
\text { I.C. moy. }=51,5 \pm 0,6\end{array}$ \\
\hline Indice $(11) * 100 /(2)$ & & 94,7 & $\begin{array}{c}\text { d'après moyennes } \\
102,0 \\
\end{array}$ \\
\hline Indice $(11) * 100 /(1)$ & 42,0 & 41,9 & $\begin{array}{l}\text { d'après moyennes } \\
44,7\end{array}$ \\
\hline Indice $(8) * 100 /(1)$ & 52,5 & 53,0 & $\begin{array}{l}\text { d'après moyennes } \\
55,2\end{array}$ \\
\hline
\end{tabular}

Les dimensions sont données en millimètres ; I.V. = intervalle de variation ;

I.C. moy. = intervalle de confiance de la moyenne; $\mathrm{n}=$ nombre de spécimens

Pour l'emplacement des diamètres mesurés, voir figures 3 et 4

B.M. $=$ British Museum ( les mesures y ont été prises par Norah Byrne)

M.P. = Muséum National d'Histoire Naturelle, laboratoire d'Anatomie Comparée

Tableau 10 - Bison bison mâles. Crâne, dimensions et indices.

Table 10 - Bison bison male skull dimensions and index values.

\begin{tabular}{|c|c|c|c|c|}
\hline & $\begin{array}{c}\text { IPGQ } \\
\text { (juvénile) }\end{array}$ & $\begin{array}{c}\text { M.B. } \\
952 \\
\text { (juvénile) }\end{array}$ & $\begin{array}{l}\text { M.P. } \\
\mathrm{n}^{\circ} 17\end{array}$ & $\begin{array}{c}\text { Bison bison bison } \\
\text { in McDonald } \\
\text { (1981, tabl. 29, p. 96) }\end{array}$ \\
\hline $\begin{array}{l}\text { (1) - Longueur saillie nuchale - } \\
\text { ligne touchant le bord antérieur } \\
\text { de chaque os incisif }\end{array}$ & 452 & 490 & 557 & $\begin{array}{l}\text { I.V. }=500-583 ; n=56 \\
\text { I.C. moy. }=535,3 \pm 2,3\end{array}$ \\
\hline $\begin{array}{l}\text { (2) - Longueur saillie nuchale - } \\
\text { épine frontale }\end{array}$ & 201 & 217 & 244 & $\begin{array}{l}\text { I.V. }=214-279 ; \mathrm{n}=106 \\
\text { I.C. moy. }=245,7 \pm 1,2\end{array}$ \\
\hline $\begin{array}{l}\text { (8) - largeur maximum au niveau } \\
\text { des orbites }\end{array}$ & 269 & 280 & 343 & $\begin{array}{l}\text { I.V. }=289-356 ; n=117 \\
\text { I.C. moy. }=324,6 \pm 1,2\end{array}$ \\
\hline $\begin{array}{l}\text { (9) - largeur minimum bord dorsal } \\
\text { du processus cornual droit - } \\
\text { bord dorsal processus cornual } \\
\text { gauche }\end{array}$ & 294 & & 319 & \\
\hline $\begin{array}{l}\text { (11) - largeur frontale minimum } \\
\text { entre les orbites et les processus } \\
\text { cornuaux }\end{array}$ & 255 & 251 & 271 & $\begin{array}{l}\text { I.V. }=237-318 ; \mathrm{n}=135 \\
\text { I.C. moy. }=271,1 \pm 1,1\end{array}$ \\
\hline $\begin{array}{l}\text { (35) - Distance entre l'apex du } \\
\text { processus cornual droit et l'apex } \\
\text { du processus cornual gauche }\end{array}$ & 610 & 526 & & $\begin{array}{l}\text { I.V. }=510-778 ; \mathrm{n}=128 \\
\text { I.C. moy. }=603,9 \pm 3,9\end{array}$ \\
\hline $\begin{array}{l}\text { (36) - Longueur du processus } \\
\text { cornual suivant la courbe } \\
\text { supérieure }\end{array}$ & & & & $\begin{array}{l}\text { I.V. }=124-270 ; n=134 \\
\text { I.C. moy. }=190,7 \pm 2,1\end{array}$ \\
\hline $\begin{array}{l}\text { (44) diamètre antéro-postérieur de la } \\
\text { couronne du processus cornual }\end{array}$ & & & & $\begin{array}{l}\text { I.V. }=67-103 ; \mathrm{n}=142 \\
\text { I.C. } \text { moy. }=83,4 \pm 0,5\end{array}$ \\
\hline Indice $(11) * 100 /(2)$ & 126,8 & 115,6 & 111,0 & $\begin{array}{c}\text { d'après moyennes } \\
110,3 \\
\end{array}$ \\
\hline $\begin{array}{l}\text { Indice }(11) * 100 /(1) \\
\text { Indice }(8) * 100 /(1)\end{array}$ & $\begin{array}{l}56,4 \\
59,5\end{array}$ & $\begin{array}{l}51,2 \\
57,1\end{array}$ & $\begin{array}{l}48,6 \\
61,6\end{array}$ & $\begin{array}{c}\text { d'après moyennes } \\
50,6 \\
\text { d'après moyennes } \\
60,6 \\
\end{array}$ \\
\hline
\end{tabular}

Les dimensions sont données en millimètres

I.V. = intervalle de variation $;$ moy = moyenne $;$ I.C. moy. = intervalle de confiance de la moyenne ;

$\mathrm{n}=$ nombre de spécimens

Pour l'emplacement des diamètres mesurés, voir figures 3 et 4

IPGQ = Institut de Préhistoire et de Géologie du Quaternaire, Université Bordeaux I

M.B. = Muséum d'Histoire Naturelle de Bordeaux

M.P. = Muséum National d'Histoire Naturelle, laboratoire d'Anatomie Comparée 
Tableau 11 - Bison priscus Habarra. Molaires supérieures, dimensions.

Table 11 - Bison priscus Habarra upper molar dimensions.

\begin{tabular}{|c|c|c|c|c|c|c|c|c|}
\hline & $\begin{array}{c}2 \mathrm{AB} \\
\text { mâle } \\
\mathrm{A} 55-\mathrm{B} 1\end{array}$ & $\begin{array}{c}\text { 4E } \\
\text { femelle } \\
\text { E12 }\end{array}$ & $\begin{array}{c}6 \mathrm{~F}(5 \mathrm{E}) \\
\text { femelle } \\
\mathrm{F} 15\end{array}$ & $\begin{array}{c}\text { 12Jd } \\
\text { mâle? } \\
\text { Jd4 }\end{array}$ & $\begin{array}{c}? \\
\text { D2 (dr.) }\end{array}$ & $\begin{array}{c}? \\
\text { D2 (g.) }\end{array}$ & $\begin{array}{c}? \\
\text { D2 (g.) }\end{array}$ & $\begin{array}{c}? \\
\text { D15(g) }\end{array}$ \\
\hline (1) Longueur de la série P2-M3 & 160 & & 156,5 & & & & & \\
\hline (2) Longueur de la série P2-P4 & 62 & & 62,5 & & & & & \\
\hline (3) P2 : DMD & & & 18,0 & & & & & \\
\hline (4) P3 : DMD & 20 & & 20,0 & & & & & 19,5 \\
\hline (5) P4 : DMD occlusal & 18,5 & & 18,0 & 21,5 & 19,5 & & & \\
\hline (6) P4 : DMD à $1 \mathrm{~cm}$ du collet & & & & & 17,0 & & & \\
\hline (7) M1 : DMD occlusal & 24,0 & & 27 & & 30,0 & 30,0 & & \\
\hline (8) M1 : DMD à $1 \mathrm{~cm}$ du collet & 24,0 & & & 32 & 28,0 & 27,5 & & \\
\hline (9) M1 : Longueur du métacône & 1,5 & & & & $33 ?$ & $33 ?$ & & \\
\hline (10) M1 Longueur du mésostyle & & & & & 28 & 28 & & \\
\hline (11) M2 : DMD occlusal & 34,0 & 26,0 & 33,0 & 35,5 & 34,5 & 34,0 & 35,0 & \\
\hline (12) M2 : DMD à $1 \mathrm{~cm}$ du collet & & & & & 32 & 32 & 32 & \\
\hline $\begin{array}{l}\text { (13) M2 : Longueur du } \\
\text { métacône }\end{array}$ & 23,5 & 17,5 & & & $49 ?$ & & & \\
\hline $\begin{array}{l}\text { (14) M2 : Longueur du } \\
\text { mésostyle }\end{array}$ & 14 & 1,5 & & & 43,0 & 43,0 & & \\
\hline (15) M3 : DMD occlusal & 41,0 & 32,0 & 36,0 & 34,0 & 33,5 & & & \\
\hline (16) M3 : DMD à $1 \mathrm{~cm}$ du collet & & & & & 34,5 & & & \\
\hline $\begin{array}{l}\text { (17) M3 : Longueur du } \\
\text { métacône }\end{array}$ & 23,5 & 15,5 & & & & & & \\
\hline $\begin{array}{l}\text { (18) M3 : Longueur du } \\
\text { mésostyle }\end{array}$ & 19,0 & & 41 & & 40,0 & & & \\
\hline
\end{tabular}

Les dimensions sont données en millimètres ;

Pour l'emplacement des diamètres mesurés, voir figure 4G ;

$\mathrm{DMD}=$ diamètre mésio-distal

Tableau 12 - Bison priscus Habarra. Mandibule, dimensions.

Table 12 - Bison priscus Habarra mandible dimensions.

\begin{tabular}{|c|c|c|c|c|c|c|}
\hline & $\begin{array}{c}1 \mathrm{AB} \\
\text { femelle } \\
\text { mand. } \\
\text { droite } \\
\mathrm{B} 18 \\
\end{array}$ & $\begin{array}{l}1 \mathrm{AB} \\
\text { femelle } \\
\text { mand. } \\
\text { gauche } \\
\text { B5 }\end{array}$ & $\begin{array}{c}2 \mathrm{AB} \\
\text { mâle } \\
\text { mand. } \\
\text { droite } \\
\text { A83 }\end{array}$ & $\begin{array}{c}\text { 3B } \\
\text { femelle } \\
\text { mand. } \\
\text { gauche } \\
\text { B88 }\end{array}$ & $\begin{array}{c}\text { 4E } \\
\text { femelle } \\
\text { mand. } \\
\text { droite } \\
\text { E38 }\end{array}$ & $\begin{array}{c}4 \mathrm{E} \\
\text { femelle } \\
\text { mand. } \\
\text { gauche } \\
\text { E73 }\end{array}$ \\
\hline $\begin{array}{l}\text { (51) du point de plus oral de la symphyse au point le plus } \\
\text { aboral de l'angle mandibulaire }\end{array}$ & & & & & 426,5 & $432 ?$ \\
\hline $\begin{array}{l}\text { (52) du point de plus oral de la symphyse au point le plus } \\
\text { aboral du condyle }\end{array}$ & & & & & 450,0 & $461 ?$ \\
\hline $\begin{array}{l}\text { (53) du bord alvéolaire distal de M3 au point le plus aboral } \\
\text { de l'angle mandibulaire }\end{array}$ & $132 ?$ & 133 & 150 & & 139,0 & $143 ?$ \\
\hline $\begin{array}{l}\text { (54) du bord alvéolaire mésial de P2 au point le plus } \\
\text { aboral de l'angle mandibulaire }\end{array}$ & $290 ?$ & 286 & 328,5 & & 246,0 & $293 ?$ \\
\hline $\begin{array}{l}\text { (55) du bord distal du foramen mentonnier au point le plus } \\
\text { aboral de l'angle mandibulaire }\end{array}$ & & 359 & & & 352 & $367 ?$ \\
\hline $\begin{array}{l}\text { (56) du point le plus oral de la symphyse au bord } \\
\text { alvéolaire distal de M3 }\end{array}$ & & & & & 289 & 290 \\
\hline $\begin{array}{l}\text { (57) du bord distal du foramen mentonnier au bord } \\
\text { alvéolaire mésial de P2 }\end{array}$ & & 71,5 & 80,5 & 62,0 & 65,9 & \\
\hline (58) Longueur du diastème C-P2 & & & & 97,0 & 124 & \\
\hline $\begin{array}{l}\text { (59) Hauteur du corps au niveau du bord alvéolaire mésial } \\
\text { de P2 }\end{array}$ & 52,5 & 48,0 & 48,5 & 45,0 & 52,8 & \\
\hline $\begin{array}{l}\text { (60) Hauteur du corps au niveau du bord alvéolaire mésial } \\
\text { de M1 }\end{array}$ & & 62 & 67,0 & 55,0 & 64,5 & 65,5 \\
\hline $\begin{array}{l}\text { (61) Hauteur du corps au niveau du bord alvéolaire mésial } \\
\text { de M3 }\end{array}$ & 80 & 79 & 78,5 & 68,0 & 70,5 & 73,0 \\
\hline $\begin{array}{l}\text { (62) Hauteur du bord ventral de l'angle mandibulaire au } \\
\text { sommet du condyle }\end{array}$ & 157 & 161 & 181,5 & & 158 & \\
\hline (13) Epaisseur du corps entre M1 et M2 & 33,5 & 33,0 & 34,0 & 31,0 & 32,0 & 32,0 \\
\hline (14) Longueur de la série P2-M3 & 158,0 & 163,0 & 174,5 & 169,5 & 151,5 & \\
\hline (15) Longueur de la série P2-P4 & 56,5 & 62,5 & 64,0 & 58,0 & 56,0 & \\
\hline (16) Longueur de la série M1-M3 & 102 & 102 & 112,5 & 108 & 99 & 93,5 \\
\hline
\end{tabular}


Tableau 13 - Bison priscus Habarra. Scapula, dimensions.

Table 13 - Bison priscus Habarra scapula dimensions.

\begin{tabular}{|l|c|c|c|c|c|}
\cline { 2 - 6 } \multicolumn{1}{c|}{} & $\begin{array}{c}1 \mathrm{AB} \\
\text { femelle }\end{array}$ & $\begin{array}{c}2 \mathrm{AB} \\
\text { mâle }\end{array}$ & $\begin{array}{c}4 \mathrm{E} \\
\text { femelle } \\
\text { f6, B60, } \\
\text { B48 } \\
\text { A22 } \\
\text { E9 } \\
\text { gauche } \\
\text { gauche }\end{array}$ & $\begin{array}{c}17 \mathrm{M} \text { ? } \\
\text { mâle } \\
\text { E55 } \\
\text { mâle } \\
\text { M11 } \\
\text { droite } \\
\text { droite }\end{array}$ \\
\hline $\begin{array}{l}\text { (1) Longueur du point le plus bas de la cavité glénoïde au sommet } \\
\text { de l'épine scapulaire }\end{array}$ & & 540 & 468 & & \\
\hline $\begin{array}{l}\text { (2) Longueur du point le plus bas de l'épine scapulaire au point le } \\
\text { plus haut du bord caudal }\end{array}$ & & & 480,5 & & \\
\hline (3) largeur maximum au sommet & & & 224 & & \\
\hline (4) largeur minimum du col & & 88,0 & 72,0 & 83,0 & 82,5 \\
\hline (5) diamètre antéro-postérieur de la cavité glénoïde & 72 & 81,5 & 71 & 79 & \\
\hline (6) Diamètre transversal maximum de la cavité glénoïde & 63 & 68 & 60 & 64 & \\
\hline (7) Diamètre antéro-postérieur total de l'extrémité ventrale & 90,0 & 107,5 & 90,5 & 105 & $110 ?$ \\
\hline
\end{tabular}

Les dimensions sont données en millimètres;

Pour l'emplacement des diamètres mesurés, voir figure $5 \mathrm{~A}$ et $\mathrm{B}$

Tableau 14 - Bison priscus Habarra. Humérus, dimensions.

Table 14 - Bison priscus Habarra humerus dimensions.

\begin{tabular}{|c|c|c|c|c|c|c|c|c|}
\hline & $\begin{array}{c}1 \mathrm{AB} \\
\text { femelle } \\
\text { A32 } \\
\text { droit }\end{array}$ & $\begin{array}{c}1 \mathrm{AB} \\
\text { femelle } \\
\text { A10 } \\
\text { gauche }\end{array}$ & $\begin{array}{l}2 \mathrm{AB} \\
\text { mâle } \\
\text { A111 } \\
\text { droit }\end{array}$ & $\begin{array}{c}2 \mathrm{AB} \\
\text { mâle } \\
\mathrm{B} 2 \\
\text { gauche }\end{array}$ & $\begin{array}{c}\text { 4E } \\
\text { femelle } \\
\text { E39 } \\
\text { gauche }\end{array}$ & $\begin{array}{c}7 \mathrm{D} \\
\text { femelle } \\
\text { D1 } \\
\text { droit } \\
\end{array}$ & $\begin{array}{c}? \\
\text { D80 } \\
\text { gauche }\end{array}$ & $\begin{array}{c}15 \mathrm{Jc} \\
\text { femelle } \\
\text { Jc61 } \\
\text { gauche }\end{array}$ \\
\hline LT Longueur totale & & & & & & & & 372,0 \\
\hline $\begin{array}{l}\text { (1) Longueur du point le plus bas de } \\
\text { l'épicondyle médial au sommet de la } \\
\text { tête articulaire }\end{array}$ & 328 & & & $\begin{array}{l}372 \\
\text { env. }\end{array}$ & 333,5 & & & 334,0 \\
\hline (2) Diamètre transversal proximal total & & & & & & & & 116,0 \\
\hline $\begin{array}{l}\text { (3) Diamètre transversal de la tête } \\
\text { articulaire }\end{array}$ & & & & & 79,0 & & & 68 \\
\hline $\begin{array}{l}\text { (4) Diamètre antéro-postérieur proximal } \\
\text { total }\end{array}$ & 134,0 & & & & $129 ?$ & & & 124,5 \\
\hline $\begin{array}{l}\text { (5) Diamètre transversal distal total, } \\
\text { perpendiculaire à l'axe principal de } \\
\text { l'os }\end{array}$ & & 107,0 & 120,5 & 121,5 & 103,5 & $92 ?$ & & 101 \\
\hline $\begin{array}{l}\text { (6) Diamètre transversal distal articulaire } \\
\text { suivant l'axe de la face articulaire }\end{array}$ & & 101,5 & $113 ?$ & 114,0 & 97,0 & 89 & & 97,0 \\
\hline $\begin{array}{l}\text { (7) Diamètre antéro-postérieur distal } \\
\text { maximum de la face médiale au } \\
\text { niveau de l'épicondyle médial }\end{array}$ & & 104,0 & & 122,5 & 110,0 & $88 ?$ & 120,5 & 101,0 \\
\hline $\begin{array}{l}\text { (8) Diamètre transversal minimum de la } \\
\text { diaphyse }\end{array}$ & 53,0 & 53,0 & & 63,0 & 50,0 & 46,5 & 54,0 & 46,5 \\
\hline Indice de robustesse $(8)^{*} 100 /(1)$ & 16,1 & & & 16,9 & 14,9 & & & 13,9 \\
\hline
\end{tabular}

Les dimensions sont données en millimètres;

Pour l'emplacement des diamètres mesurés, voir figure $6 \mathrm{~A}$ et $\mathrm{B}$ 
Tableau 15 - Bison priscus Habarra. Radius et ulna, dimensions et indice. Table 15 - Bison priscus Habarra radius and ulna dimensions and index values.

\begin{tabular}{|c|c|c|c|c|c|c|c|c|c|}
\hline & $\begin{array}{c}\mathrm{AB} \\
\text { femelle } \\
\text { A11 } \\
\text { droit }\end{array}$ & $\begin{array}{c}1 \mathrm{AB} \\
\text { femelle } \\
\text { A37 } \\
\text { gauche }\end{array}$ & \begin{tabular}{c|}
$2 \mathrm{AB}$ \\
mâle \\
$\mathrm{A} 20$ \\
droit
\end{tabular} & $\begin{array}{c}2 \mathrm{AB} \\
\text { mâle } \\
\mathrm{B} 4 \\
\text { gauche }\end{array}$ & $\begin{array}{c}\mathrm{E} \\
\text { femelle } \\
\mathrm{E} 47 \\
\text { droit }\end{array}$ & $\begin{array}{c}7 \mathrm{D} \\
\text { femelle } \\
\mathrm{D} 7 \\
\text { gauche }\end{array}$ & $\begin{array}{c}\text { 8D? } \\
\text { mâle } \\
\text { D16 } \\
\text { gauche }\end{array}$ & $\begin{array}{c}17 \mathrm{M} \\
\text { mâle } \\
\text { M4 } \\
\text { gauche }\end{array}$ & $\begin{array}{c}18 \\
\text { mâle } \\
\alpha 1 \\
\text { gauche }\end{array}$ \\
\hline $\begin{array}{l}\text { Longueur de l'ensemble radio- } \\
\text { ulnaire }\end{array}$ & 431,5 & & & & & & & & \\
\hline (1) Longueur totale du radius & 333,0 & & 378,0 & 380,0 & 355,5 & 340,0 & 362 & & \\
\hline $\begin{array}{l}\text { (2) Diamètre transversal } \\
\text { proximal total }\end{array}$ & 102,0 & 105,0 & 120,5 & 118,5 & 102,5 & 96,5 & 105,5 & 122,5 & 122,5 \\
\hline $\begin{array}{l}\text { (3) Diamètre transversal de la } \\
\text { face articulaire proximale } \\
\text { du radius }\end{array}$ & 97,0 & 98,0 & 109,0 & 108,0 & 95,0 & 88,5 & 102,0 & 107,0 & 109,0 \\
\hline $\begin{array}{l}\text { (4) Diamètre antéro-postérieur } \\
\text { proximal total du radius }\end{array}$ & 52 & 53,0 & 60,0 & 60,0 & 58,5 & 50,5 & 54,0 & 61 & 59,5 \\
\hline $\begin{array}{l}\text { (5) Diamètre transversal distal } \\
\text { total }\end{array}$ & 93,0 & & 111,0 & 108,0 & 105,0 & 86,5 & 105,5 & & \\
\hline $\begin{array}{l}\text { (6) Diamètre transversal } \\
\text { minimum du radius }\end{array}$ & 52,0 & 52,0 & 64,0 & 63,5 & 51,5 & 46,0 & 57,5 & 61,5 & 61,5 \\
\hline $\begin{array}{l}\text { Indice de robustesse } \\
(6)^{*} 100 /(1)\end{array}$ & 15,6 & & 16,9 & 16,7 & 14,4 & 13,5 & 15,8 & & \\
\hline
\end{tabular}

Les dimensions sont données en millimètres ;

Pour l'emplacement des diamètres mesurés, voir figure $6 \mathrm{C}$ et $\mathrm{E}$

Tableau 16 - Bison priscus Habarra. Ulna, dimensions.

Table 16 - Bison priscus Habarra ulna dimensions.

\begin{tabular}{|c|c|c|c|c|c|c|c|c|c|}
\cline { 2 - 10 } \multicolumn{1}{c|}{} & $\begin{array}{c}1 \mathrm{AB} \\
\text { femelle } \\
\mathrm{A} 11 \\
\text { droit }\end{array}$ & $\begin{array}{c}1 \mathrm{AB} \\
\text { femelle } \\
\mathrm{A} 6 \\
\text { gauche }\end{array}$ & $\begin{array}{c}2 \mathrm{AB} \\
\text { mâle } \\
\mathrm{B} 31 \\
\mathrm{droit}\end{array}$ & $\begin{array}{c}2 \mathrm{AB} \\
\text { mâle } \\
\mathrm{B} 4 \\
\text { gauche }\end{array}$ & $\begin{array}{c}4 \mathrm{E} \\
\text { femelle } \\
\mathrm{E} 47 \\
\text { droit }\end{array}$ & $\begin{array}{c}4 \mathrm{E} \\
\text { femelle } \\
\mathrm{E} 29 \\
\text { gauche }\end{array}$ & $\begin{array}{c}8 \mathrm{D} ? \\
\text { mâle } \\
\mathrm{D} 30-31 \\
\text { gauche }\end{array}$ & $\begin{array}{c}17 \mathrm{M} \\
\text { mâle } \\
\text { M4 } \\
\text { gauche }\end{array}$ & $\begin{array}{c}18 \\
\text { mâle } \\
\text { a1 } \\
\text { gauche }\end{array}$ \\
\hline $\begin{array}{c}\text { (1) Longueur de l'olécrâne } \\
\text { (du bec au sommet) }\end{array}$ & 131 & 130 & 164 & & 128 & & 143,5 & 166,5 & 167,0 \\
\hline $\begin{array}{c}\text { (2) Diamètre antéro- } \\
\text { postérieur au niveau du } \\
\text { bec }\end{array}$ & 97,5 & 97,5 & 108 & 109,0 & 92,5 & 92,0 & 104,5 & 114,0 & 113,0 \\
\hline $\begin{array}{c}\text { (3) Diamètre antéro- } \\
\text { postérieur minimum }\end{array}$ & 74,5 & 75,0 & 85,5 & 86,5 & 75,5 & 75,0 & 81,5 & & \\
\hline $\begin{array}{c}\text { (4) Diamètre transversal de } \\
\text { la face articulaire pour } \\
\text { l'humérus }\end{array}$ & 30 & 31,5 & $37 ?$ & 35,5 & 32,0 & 31,5 & 30,5 & 38,5 & 39,5 \\
\hline $\begin{array}{c}\text { (5) Diamètre transversal au } \\
\text { niveau de la face } \\
\text { articulaire pour le radius }\end{array}$ & 60 & 59,5 & & 67,0 & 62,0 & 62,0 & 61,0 & & \\
\hline
\end{tabular}

Les dimensions sont données en millimètres ;

Pour l'emplacement des diamètres mesurés, voir figure $6 \mathrm{C}$ et $\mathrm{D}$

Tableau 17 - Bison priscus Habarra. Pyramidal, dimensions.

Table 17 - Bison priscus Habarra pyramidal dimensions.

\begin{tabular}{|c|c|c|c|c|c|}
\hline & $\begin{array}{c}1 \mathrm{AB} \\
\text { femelle } \\
\text { A40 } \\
\text { gauche }\end{array}$ & $\begin{array}{l}2 \mathrm{AB} \\
\text { mâle } \\
\text { A102 } \\
\text { droit } \\
\end{array}$ & $\begin{array}{c}\text { 2AB } \\
\text { mâle } \\
\text { B46 } \\
\text { gauche }\end{array}$ & $\begin{array}{c}\mathrm{EE} \\
\text { femelle } \\
\text { E73 } \\
\text { droit } \\
\end{array}$ & $\begin{array}{c}\mathrm{E} \\
\text { femelle } \\
\text { E63 } \\
\text { gauche }\end{array}$ \\
\hline (1) Diamètre antéro-postérieur & 42,5 & 50,5 & 50,5 & 45,0 & 44,5 \\
\hline (2) Diamètre transversal & 27,5 & 32,0 & 34,0 & 28,0 & 28,0 \\
\hline (3) Hauteur maximum & 47,5 & 53,0 & 55,0 & 47,0 & 48,0 \\
\hline
\end{tabular}

Les dimensions sont données en millimètres ;

Pour l'emplacement des diamètres mesurés, voir figure $8 \mathrm{~A}$ 
Tableau 18 - Bison priscus Habarra. Semilunaire, dimensions. Table 18 - Bison priscus Habarra semilunar dimensions.

\begin{tabular}{|c|c|c|c|c|}
\hline & $\begin{array}{c}1 \mathrm{AB} \\
\text { femelle } \\
\text { A104 } \\
\text { gauche }\end{array}$ & $\begin{array}{l}2 \mathrm{AB} \\
\text { mâle } \\
\text { A103 } \\
\text { droit }\end{array}$ & $\begin{array}{c}\mathrm{EE} \\
\text { femelle } \\
\text { E73 } \\
\text { droit }\end{array}$ & $\begin{array}{c}3 \mathrm{~B} ? \\
\text { femelle } \\
\text { A4 } \\
\text { gauche }\end{array}$ \\
\hline (1) Diamètre antéro-postérieur & & & 54,0 & 48,5 \\
\hline (2) Diamètre transversal dorsal & 34 & 37,5 & 33 & 32,5 \\
\hline (3) Hauteur dorsale & 32,0 & 36,0 & 34,5 & 31,0 \\
\hline (4) Diamètre transversal minimum dorsal & & 26 & & \\
\hline
\end{tabular}

Les dimensions sont données en millimètres ;

Pour l'emplacement des diamètres mesurés, voir figure $8 \mathrm{~B}$

Tableau 19 - Bison priscus Habarra. Scaphoïde, dimensions.

Table 19 - Bison priscus Habarra scaphoid dimensions.

\begin{tabular}{|l|c|c|}
\cline { 2 - 3 } \multicolumn{1}{c|}{} & $2 \mathrm{AB}$ & $4 \mathrm{E}$ \\
mâle & femelle \\
\cline { 2 - 3 } \multicolumn{1}{c|}{} & $\mathrm{B} 24$ & $\mathrm{E} 73$ \\
(1) Diamètre antéro-postérieur & 63,5 & 57,5 \\
\hline (2) Diamètre transversal total & 41 & 36,5 \\
\hline (3) Hauteur & 42,5 & 40,0 \\
\hline
\end{tabular}

Les dimensions sont données en millimètres ;

Pour l'emplacement des diamètres mesurés, voir figure $8 \mathrm{E}$

Tableau 20 - Bison priscus Habarra. Unciforme, dimensions.

Table 20 - Bison priscus Habarra unciform dimensions.

\begin{tabular}{|l|c|c|c|}
\cline { 2 - 4 } \multicolumn{1}{c|}{} & $2 \mathrm{AB}$ & $4 \mathrm{E}$ & $8 \mathrm{D} ?$ \\
& mâle & femelle & mâle \\
$\mathrm{A} 103$ & $\begin{array}{c}\mathrm{E} 73 \\
\text { (1) Diamètre antéro-postérieur }\end{array}$ & $\begin{array}{c}\mathrm{D} 87 \\
\text { droit }\end{array}$ & $\begin{array}{c}\text { droit } \\
\text { gauche }\end{array}$ \\
\hline (2) Diamètre transversal & 35,0 & 37,5 & \\
\hline (3) Hauteur maximum & 34 & 31,5 & 44,5 \\
\hline
\end{tabular}

Les dimensions sont données en millimètres ;

Pour l'emplacement des diamètres mesurés, voir figure $8 \mathrm{D}$ 
Tableau 21 - Bison priscus Habarra. Capitato-trapézoïde, dimensions. Table 21 - Bison priscus Habarra capitato-trapezoid dimensions.

\begin{tabular}{|l|c|cc|}
\cline { 2 - 4 } \multicolumn{1}{c|}{} & $\begin{array}{c}2 \mathrm{AB} \\
\text { mâle }\end{array}$ & $\begin{array}{c}2 \mathrm{AB} \\
\text { mâle }\end{array}$ & $\begin{array}{c}\text { 4E } \\
\text { femelle }\end{array}$ \\
& $\begin{array}{c}\text { A100 } \\
\text { droit }\end{array}$ & $\begin{array}{c}\mathrm{B} 46 \\
\text { gauche }\end{array}$ & $\begin{array}{c}\text { E73 } \\
\text { droit }\end{array}$ \\
\hline (1) Diamètre antéro-postérieur & 49,5 & 49,5 & 45,0 \\
\hline (2) Diamètre transversal & 52,5 & 53,5 & 45,5 \\
\hline (3) Hauteur dorsale & 23 & 22,5 & 20,5 \\
\hline (4) Hauteur latérale maximum & 27,5 & 28,0 & 27,0 \\
\hline
\end{tabular}

Les dimensions sont données en millimètres ;

Pour l'emplacement des diamètres mesurés, voir figure $8 \mathrm{C}$

Tableau 22 - Bison priscus. Métacarpe, dimensions et indices.

Table 22 - Bison priscus Habarra metacarpal dimensions and index values.

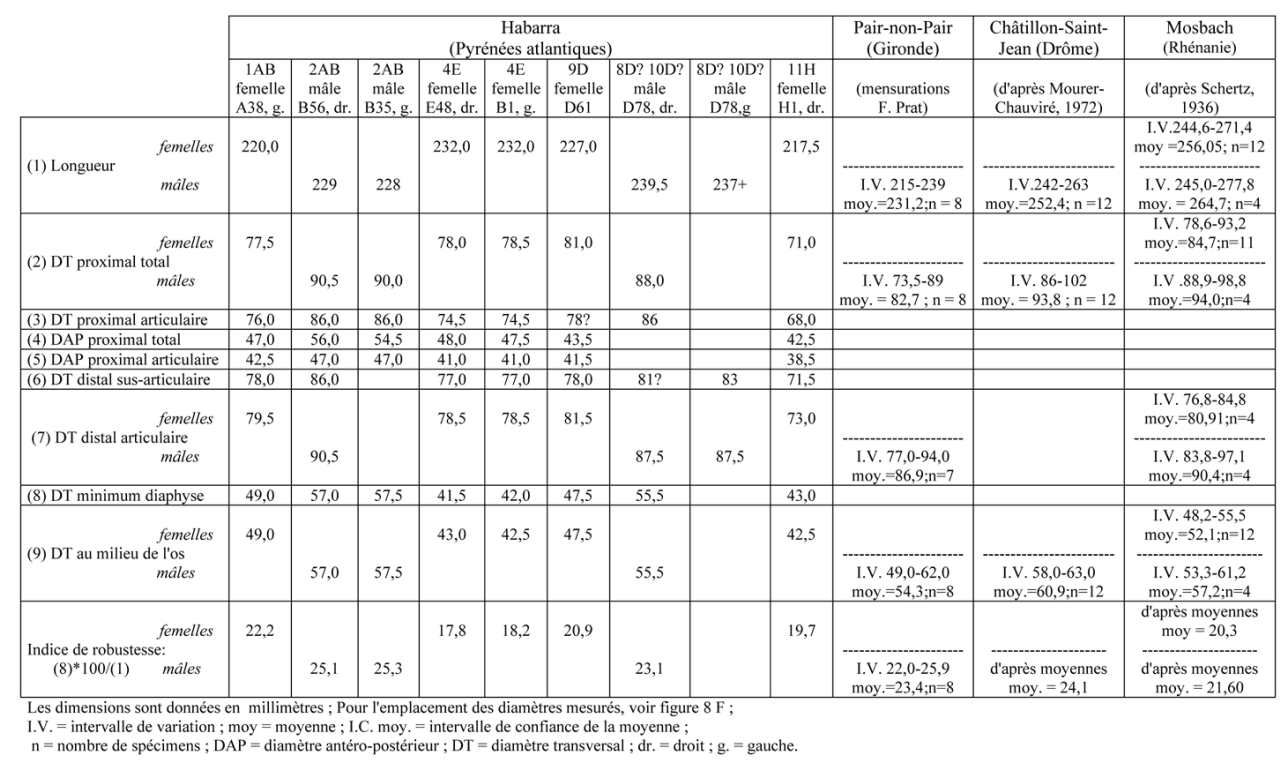

Tableau 23 - Bison priscus Habarra. Premières phalanges antérieures, dimensions. Table 23 - Bison priscus Habarra: Dimensions of the first phalanges of the forelimb.

\begin{tabular}{|c|c|c|c|c|c|c|}
\hline & $\begin{array}{c}1 \mathrm{AB} \\
\text { femelle } \\
\mathrm{A} 45 \\
\end{array}$ & \begin{tabular}{|c|}
$1 \mathrm{AB}$ \\
femelle \\
$\mathrm{A} 44$ \\
\end{tabular} & $\begin{array}{c}1 \mathrm{AB} \\
\text { femelle } \\
\mathrm{A} 41 \\
\end{array}$ & \begin{tabular}{c|}
$2 \mathrm{AB}$ \\
mâle \\
$\mathrm{B} 30$ \\
\end{tabular} & \begin{tabular}{|c|}
$2 \mathrm{AB}$ \\
mâle \\
$\mathrm{B} 60$ \\
\end{tabular} & \begin{tabular}{|l} 
AB \\
mâle \\
B62 \\
\end{tabular} \\
\hline (1) Longueur totale & 72,5 & 72,5 & & 79,5 & 81 & \\
\hline (2) Longueur axiale & 62,5 & 62 & & 72,0 & & \\
\hline (3) Diamètre transversal proximal total & 38,0 & 39,5 & & 46,5 & 47,0 & 46,5 \\
\hline (4) Diamètre antéro-postérieur proximal total & 38,0 & 35,5 & & 45,0 & & 47,5 \\
\hline (5) Diamètre transversal distal articulaire & 38,5 & 39,0 & & 46,0 & 45,5 & \\
\hline (6) Diamètre antéro-postérieur distal total & 27,0 & 26,5 & 25,5 & & 32,0 & \\
\hline (7) Diamètre transversal minimum de la diaphyse & 37,0 & 36,0 & 36,5 & 42,0 & 41,0 & \\
\hline
\end{tabular}

Les dimensions sont données en millimètres ;

Pour l'emplacement des diamètres mesurés, voir figure $8 \mathrm{G}$ 
Tableau 24 - Bison priscus Habarra. Deuxième phalanges antérieures, dimensions. Table 24 - Bison priscus Habarra: Dimensions of the second phalanges of the forelimb.

\begin{tabular}{|l|c|c|c|c|c|c|}
\cline { 2 - 7 } \multicolumn{1}{c|}{} & $\begin{array}{c}\text { 1AB } \\
\text { femelle } \\
\text { A43 }\end{array}$ & $\begin{array}{c}\text { 2AB } \\
\text { mâle } \\
\text { A101 }\end{array}$ & $\begin{array}{c}\text { 2AB } \\
\text { mâle } \\
\text { A85 }\end{array}$ & $\begin{array}{c}\text { 2AB } \\
\text { mâle } \\
\text { B62 }\end{array}$ & $\begin{array}{c}2 \mathrm{AB} \\
\text { mâle } \\
\text { B62 }\end{array}$ & $\begin{array}{c}\text { 2AB? } \\
\text { mâle } \\
\text { B13 } \\
\text { antér? }\end{array}$ \\
\hline (1) Longueur totale & 50,5 & 55,0 & 56,5 & 53,5 & 58,0 & 50 \\
\hline (3) Diamètre transversal proximal total & 40,0 & 49,5 & 49,5 & 46,0 & 48,5 & \\
\hline (4) Diamètre antéro-postérieur proximal & 39,0 & 47 & 48,0 & & & 38 \\
\hline (5) Diamètre transversal distal & 37,0 & 46,0 & 46,5 & 45,5 & & \\
\hline (6) Diamètre antéro-postérieur distal & 34,5 & 42,0 & 45,0 & 42,0 & 43,5 & 35,5 \\
\hline (7) Diamètre transversal de la région moyenne & 37,5 & & 43,0 & 37,5 & 38,5 & \\
\hline
\end{tabular}

Les dimensions sont données en millimètres;

Pour l'emplacement des diamètres mesurés, voir figure $8 \mathrm{H}$

Tableau 25 - Bison priscus Habarra. Os coxal, dimensions.

Table 25 - Bison priscus Habarra innominate dimensions.

\begin{tabular}{|l|c|c|}
\cline { 2 - 3 } \multicolumn{1}{c|}{} & $\begin{array}{c}1 \mathrm{AB} \\
\text { femelle } \\
\text { A1 }\end{array}$ & $\begin{array}{c}6 \mathrm{~F}(5 \mathrm{E} ?) \\
\text { femelle } \\
\text { F2 } \\
\text { droit }\end{array}$ \\
\hline $\begin{array}{l}\text { (1) Distance entre le point le plus crânial de l'acétabulum et le point correspondant au } \\
\text { maximum de la concavité de la crête iliaque }\end{array}$ & & $\# 260$ \\
\hline (2) Largeur minimum du col de l'ilium & 52,5 & 57,5 \\
\hline (3) Epaisseur minimum du col de l'ilium & 34,0 & 37,0 \\
\hline (4) Largeur minimum de la table de l'ischium & & 72,0 \\
\hline (5) Diamètre antéro-postérieur maximum de l'acétabulum & & 92,0 \\
\hline (6) Diamètre antéro-postérieur maximum de la surface semi-lunaire & & 89,0 \\
\hline (7) Diamètre dorso-ventral de l'acétabulum (perpendiculaire au précédent) & & 84,0 \\
\hline (8) Plus grand diamètre du trou ovalaire & & 115,0 \\
\hline (9) Diamètre dorso-ventral maximum du trou ovalaire & & 67,0 \\
\hline $\begin{array}{l}\text { (10) Distance entre le point le plus dorsal de la crête sciatique et le point le plus caudal du } \\
\text { bord de l'acétabulum }\end{array}$ & & 114 \\
\hline
\end{tabular}

Les dimensions sont données en millimètres ;

Pour l'emplacement des diamètres mesurés, voir figure $5 \mathrm{C}$

Tableau 26 - Bison priscus Habarra. Fémur, dimensions et indice. Table 26 - Bison priscus Habarra femur dimensions and index values.

\begin{tabular}{|c|c|c|c|c|c|c|c|c|}
\hline & $\begin{array}{c}2 \mathrm{AB} \\
\text { mâle } \\
\mathrm{A} 114 \\
\text { droit }\end{array}$ & $\begin{array}{c}2 \mathrm{AB} \\
\text { mâle } \\
\mathrm{A} 33 \\
\text { gauche }\end{array}$ & $\begin{array}{c}4 \mathrm{E} \\
\text { femelle } \\
\mathrm{E} 2 \\
\text { droit }\end{array}$ & $\begin{array}{c}5 \mathrm{E} \\
\text { femelle } \\
\text { Essbloc } \\
\text { droit }\end{array}$ & $\begin{array}{c}\text { 8D? } \\
\text { mâle } \\
\text { D87 } \\
\text { gauche }\end{array}$ & $\begin{array}{c}10 \mathrm{D} \\
\text { mâle } \\
\text { D56- } \\
77 \\
\text { droit }\end{array}$ & $\begin{array}{c}15 \mathrm{Jc} \\
\text { femelle } \\
\text { Jc45 } \\
\text { droit }\end{array}$ & $\begin{array}{l}15 \mathrm{Jc} \\
\text { femelle } \\
\mathrm{Jc} 43 \\
\text { gauche }\end{array}$ \\
\hline \begin{tabular}{|l} 
(1) Longueur du sommet de la tête à la \\
base de la lèvre médiale de la trochlée
\end{tabular} & 471 & 473 & 427,0 & & & & & \\
\hline \begin{tabular}{|l|} 
(2) Diamètre transversal proximal total \\
\end{tabular} & & & & & 165,0 & & $144 ?$ & \\
\hline (3) Daimètre antéro-postérieur tête & 66,0 & 66,5 & 62,0 & & & 65,5 & $55 ?$ & \\
\hline (4) Diamètre transversal distal total & & 137,5 & 120,0 & 117,5 & & & & 111 \\
\hline $\begin{array}{l}\text { (5) Diamètre transversal de la trochlée à sa } \\
\text { base }\end{array}$ & & 56,0 & 44,5 & 44,5 & & & & 41,5 \\
\hline $\begin{array}{l}\text { (6) Longueur de la lèvre médiale de la } \\
\text { trochlée }\end{array}$ & & $125 ?$ & & 118,0 & & & & 106,0 \\
\hline $\begin{array}{l}\text { (7) Diamètre transversal minimum de la } \\
\text { diaphyse }\end{array}$ & 55,0 & 54,5 & 45,5 & 46,5 & 52,7 & & 46,0 & 46,0 \\
\hline (8) Grand diamètre du condyle médial & 81,5 & 80,5 & 74,5 & 72,0 & & & & 69,5 \\
\hline (9) Petit diamètre du condyle médial & $55 ?$ & 57,5 & 51,0 & 45,5 & & & & 45,0 \\
\hline Indice de robustesse $(7) * 100 /(1)$ & 11,6 & 11,5 & 10,6 & & & & & \\
\hline
\end{tabular}

Les dimensions sont données en millimètres ;

Pour l'emplacement des diamètres mesurés, voir figure 7 A, B, C 
Tableau 27 - Bison priscus Habarra. Tibia, dimensions et indices. Table 27 - Bison priscus Habarra tibia dimensions and index values.

\begin{tabular}{|c|c|c|c|c|c|c|c|}
\hline & $\begin{array}{c}1 \mathrm{AB} \\
\text { femelle } \\
\text { A15 } \\
\text { droit }\end{array}$ & \begin{tabular}{|c|}
$1 \mathrm{AB}$ \\
femelle \\
A16 \\
gauche
\end{tabular} & $\begin{array}{l}2 \mathrm{AB} \\
\text { mâle } \\
\text { A29 } \\
\text { droit }\end{array}$ & $\begin{array}{c}2 \mathrm{AB} \\
\text { mâle } \\
\text { A49 } \\
\text { gauche }\end{array}$ & $\begin{array}{c}4 \mathrm{E} \\
\text { femelle } \\
\text { E1 } \\
\text { droit }\end{array}$ & \begin{tabular}{|c}
$\mathrm{E}$ \\
femelle \\
E23 \\
droit
\end{tabular} & $\begin{array}{c}\text { 8D } \\
\text { mâle } \\
\text { D91 } \\
\text { gauche }\end{array}$ \\
\hline (1) Longueur totale & 402,0 & 399,0 & 476,0 & & 436,0 & 420 & 458 \\
\hline (2) Diamètre transversal proximal & & & 145,5 & & 124,0 & & 140,0 \\
\hline (3) Diamètre antéro-postérieur proximal total & & & $135 ?$ & & $115 ?$ & & 123 \\
\hline (4) Diamètre transversal distal total & 79,5 & $79,5 ?$ & 93,5 & 90,5 & 77,5 & 82,5 & 85,5 \\
\hline (5) Diamètre antéro-postérieur distal total & 59 & 60,0 & 65,5 & 65,0 & 57,0 & 57,5 & 64,0 \\
\hline (6) Diamètre transversal minimum de la diaphyse & 51,0 & 52,0 & 62,0 & 63,0 & 50,0 & 50,0 & 56,6 \\
\hline (7) Diamètre transversal au milieu de l'os & 56,5 & 55,5 & & & 53,5 & & 62,0 \\
\hline Indice $(6)^{*} 100 /(1)$ & 12,6 & 13,0 & 13,0 & & 11,2 & 11,9 & 12,0 \\
\hline Indice $(7) * 100 /(1)$ & 14,0 & 15,0 & & & & & 13,5 \\
\hline
\end{tabular}

Les dimensions sont données en millimètres ;

Pour l'emplacement des diamètres mesurés, voir figure 7 D, E, F

Tableau 28 - Bison priscus Habarra. Rotule, dimensions. Table 28 - Bison priscus Habarra patella dimensions.

\begin{tabular}{|l|c|c|}
\cline { 2 - 3 } \multicolumn{1}{c|}{} & $4 \mathrm{E}$ & $4 \mathrm{E}$ \\
femelle & femelle \\
E18 & E25 \\
& droit & gauche \\
\hline Diamètre proximo-distal & 79,5 & 77,5 \\
\hline Diamètre transversal & 65,0 & 62,5 \\
\hline
\end{tabular}

Les dimensions sont données en millimètres.

Tableau 29 - Bison priscus Habarra. Os malléolaire, dimensions.

Table 29 - Bison priscus Habarra fibula dimensions.

\begin{tabular}{|l|c|c|}
\cline { 2 - 3 } \multicolumn{1}{c|}{} & $2 \mathrm{AB}$ & 4E \\
\multicolumn{1}{c|}{} & mâle & femelle \\
& $\mathrm{A} 63$ & $\mathrm{E} 25$ \\
(1) Diamètre antéro-postérieur & gauche \\
\hline (2) Diamètre transversal & 48,0 & 43,5 \\
\hline (3) Hauteur au niveau de la région plantaire & 26,0 & 22,5 \\
\hline
\end{tabular}

Les dimensions sont données en millimètres. 
Tableau 30 - Bison priscus Habarra. Cubo-naviculaire, dimensions. Table 30 - Bison priscus Habarra naviculo-cuboid dimensions.

\begin{tabular}{|c|c|c|c|c|c|c|c|}
\hline & $\begin{array}{c}1 \mathrm{AB} \\
\text { femelle } \\
\text { A18 } \\
\text { gauche }\end{array}$ & \begin{tabular}{|l|}
$2 \mathrm{AB}$ \\
mâle \\
$\mathrm{A} 53$ \\
droit
\end{tabular} & $\begin{array}{c}2 \mathrm{AB} \\
\text { mâle } \\
\text { A117 } \\
\text { gauche }\end{array}$ & $\begin{array}{c}4 \mathrm{E} \\
\text { femelle } \\
\text { E73 } \\
\text { droit }\end{array}$ & $\begin{array}{c}4 \mathrm{E} \\
\text { femelle } \\
\text { E37 } \\
\text { gauche }\end{array}$ & $\begin{array}{c}\mathrm{E} \\
\text { femelle } \\
\text { E17 } \\
\text { droit }\end{array}$ & \begin{tabular}{|l|} 
8D? \\
mâle \\
D51 \\
droit \\
\end{tabular} \\
\hline (1) Diamètre antéro-postérieur & 66,5 & 76,0 & 71,5 & 65,5 & 69,5 & 66,5 & 70,0 \\
\hline (2) Diamètre transversal & 69,0 & 83,0 & 82 & 70,0 & 75,0 & 69,0 & 77,0 \\
\hline $\begin{array}{l}\text { (3) Longueur facette articulaire distale latéro- } \\
\text { plantaire }\end{array}$ & 18,5 & 30,0 & 28,0 & 22,0 & 19,0 & 23 & \\
\hline (4) largeur facette articulaire distale latéro-plantaire & 10,0 & 12,5 & 12,0 & 11,2 & 11,0 & 10,5 & 11,0 \\
\hline
\end{tabular}

Les dimensions sont données en millimètres.

Tableau 31 - Bison priscus Habarra. Grand cunéiforme, dimensions. Table 31 - Bison priscus Habarra greater cuneiform dimensions.

\begin{tabular}{|l|c|c|c|c|}
\cline { 2 - 5 } \multicolumn{1}{c|}{} & $2 \mathrm{AB}$ & $4 \mathrm{E}$ & $5 \mathrm{E}$ & $5 \mathrm{E}$ \\
\multicolumn{1}{c|}{} & mâle & femelle & femelle & femelle \\
& A62 & E73 & E16 & E10 \\
\cline { 2 - 5 } \multicolumn{1}{c|}{} & droit & droit & droit & gauche \\
\hline (1) Diamètre antéro-postérieur & 51,5 & 46,5 & 45,0 & 43,5 \\
\hline (2) Diamètre transversal & 33,5 & 29,5 & 26,5 & 25,5 \\
\hline (3) Hauteur & 22,0 & 20,5 & 18,5 & 18,5 \\
\hline
\end{tabular}

Les dimensions sont données en millimètres.

Tableau 32 - Bison priscus Habarra. Talus, dimensions et indice. Table 32 - Bison priscus Habarra talus dimensions and index values.

\begin{tabular}{|l|c|c|c|c|c|c|c|c|c|}
\cline { 2 - 10 } \multicolumn{1}{c|}{} & $\begin{array}{c}1 \mathrm{AB} \\
\text { femelle } \\
\mathrm{A23} \\
\text { droit }\end{array}$ & $\begin{array}{c}1 \mathrm{AB} \\
\text { femelle } \\
\mathrm{A} 24 \\
\text { gauche }\end{array}$ & $\begin{array}{c}2 \mathrm{AB} \\
\text { mâle } \\
\mathrm{A} 67 \\
\text { droit }\end{array}$ & $\begin{array}{c}2 \mathrm{AB} \\
\text { mâle } \\
\text { A116 } \\
\text { gauche }\end{array}$ & $\begin{array}{c}4 \mathrm{E} \\
\text { femelle } \\
\text { E62 } \\
\text { droit }\end{array}$ & $\begin{array}{c}4 \mathrm{E} \\
\text { femelle } \\
\text { E37 } \\
\text { gauche }\end{array}$ & $\begin{array}{c}5 \mathrm{E} \\
\text { femelle } \\
\text { E21 } \\
\text { droit }\end{array}$ & $\begin{array}{c}5 \mathrm{E} \\
\text { femelle } \\
\text { E19 } \\
\text { gauche }\end{array}$ & $\begin{array}{c}\text { 7D? } \\
\text { femelle } \\
\mathrm{D} 48 \\
\text { droit }\end{array}$ \\
\hline $\begin{array}{c}\text { (1) Longueur totale (=Hauteur } \\
\text { totale) }\end{array}$ & 84,5 & 82 & 89,5 & 89,0 & 89,5 & 89,5 & $85,5 ?$ & $83 ?$ & 81,0 \\
\hline (2) Longueur axiale & 65,0 & 63,5 & 67,0 & 67,0 & 68,5 & 68,5 & & 68,5 & 63,0 \\
\hline (3) Diamètre transversal total & 58,0 & 56,5 & 67,0 & 66,0 & 58,5 & & 57,5 & 60 & 56,5 \\
\hline $\begin{array}{c}\text { (4) Diamètre transversal trochlée } \\
\text { proximale (au sommet) }\end{array}$ & 51,0 & 49,5 & 55,0 & 55,5 & 51,0 & 53,0 & 51,0 & 52,0 & 49,0 \\
\hline $\begin{array}{c}\text { (5) Diamètre transversal } \\
\text { maximum de la trochlée } \\
\text { distale }\end{array}$ & 57,5 & & 63,0 & 62,0 & 53,0 & 55,5 & 54,5 & 57,5 & 53,5 \\
\hline $\begin{array}{c}\text { (6) Diamètre antéro-postérieur } \\
\text { total }\end{array}$ & 47,5 & 46,5 & 52,5 & 52,5 & 49,0 & 50,0 & 49,0 & 50,0 & 45,0 \\
\hline Indice (3)*100/(1) & 68,6 & 68,4 & 74,8 & 74,5 & 65,3 & & 67,2 & 72,2 & 69,7 \\
\hline
\end{tabular}

Les dimensions sont données en millimètres

Pour l'emplacement des diamètres mesurés, voir figure $7 \mathrm{G}$ 
Tableau 33 - Bison priscus Habarra. Calcanéum, dimensions. Table 33 - Bison priscus Habarra calcaneus dimensions.

\begin{tabular}{|l|c|c|c|c|c|c|c|c|c|}
\cline { 2 - 10 } \multicolumn{1}{c|}{} & $\begin{array}{c}1 \mathrm{AB} \\
\text { femelle } \\
\mathrm{A} 12 \\
\text { droit }\end{array}$ & $\begin{array}{c}1 \mathrm{AB} \\
\text { femelle } \\
\text { A39 } \\
\text { gauche }\end{array}$ & $\begin{array}{c}2 \mathrm{AB} \\
\text { mâle } \\
\mathrm{A} 46 \\
\text { droit }\end{array}$ & $\begin{array}{c}2 \mathrm{AB} \\
\text { mâle } \\
\text { A88 } \\
\text { gauche }\end{array}$ & $\begin{array}{c}4 \mathrm{E} \\
\text { femelle } \\
\text { E74 } \\
\text { droit }\end{array}$ & $\begin{array}{c}4 \mathrm{E} \\
\text { femelle } \\
\text { E37 } \\
\text { gauche }\end{array}$ & $\begin{array}{c}5 \mathrm{E} \\
\text { femelle } \\
\text { E16 } \\
\text { droit }\end{array}$ & $\begin{array}{c}7 \mathrm{D} ? \\
\text { femelle } \\
\text { D86 } \\
\text { droit }\end{array}$ & $\begin{array}{c}8 \mathrm{D} ? \\
\text { mâle } \\
\text { DB2 } \\
\text { droit }\end{array}$ \\
\hline (1) Longueur totale & 159,5 & & 191,0 & 187,0 & 166,5 & 168,0 & 171,0 & 161,0 & 173,5 \\
\hline (2) Diamètre transversal total & 54,0 & 60,0 & 68,0 & 68,0 & 58,5 & 60,0 & 59,0 & 54,0 & 61,0 \\
\hline $\begin{array}{c}\text { (3) Diamètre transversal de la } \\
\text { tubérosité proximale }\end{array}$ & & & 51,5 & & 43,5 & 44,5 & 43,5 & 43,0 & 51,5 \\
\hline $\begin{array}{c}\text { (4) Diamètre antéro-postérieur de } \\
\text { la tubérosité proximale }\end{array}$ & 43,0 & & & & 44,5 & 45,0 & 49,5 & 44,5 & 54,0 \\
\hline $\begin{array}{c}\text { (5) Diamètre antéro-postérieur } \\
\text { total }\end{array}$ & 68,0 & 70,0 & 75,5 & 73,5 & 69,0 & 70,0 & 70,5 & 69,0 & \\
\hline $\begin{array}{c}\text { (6) Longueur de la facette } \\
\text { articulaire pour l'os } \\
\text { malléolaire }\end{array}$ & 36,5 & 36,5 & 41,0 & & 40,0 & 44,3 & 39,5 & 35,0 & 35,5 \\
\hline $\begin{array}{c}\text { (7) Largeur de la facette } \\
\text { articulaire pour l'os } \\
\text { malléolaire }\end{array}$ & 17,5 & 19,5 & 22,5 & & 19,5 & 19,5 & 21,0 & 20,0 & 19,0 \\
\hline $\begin{array}{c}\text { (8) Longueur de la facette } \\
\text { articulaire pour le cubo- } \\
\text { naviculaire }\end{array}$ & 43,0 & 46,0 & 53,0 & 53 & 48,0 & 48,0 & 48,0 & 46,0 & 44,5 \\
\hline
\end{tabular}

Les dimensions sont données en millimètres.

Tableau 34 - Bison priscus. Métatarse, dimensions et indices. Table 34 - Bison priscus Habarra metatarsal dimensions and index values.

\begin{tabular}{|c|c|c|c|c|c|c|c|c|c|c|c|c|}
\hline & \multicolumn{10}{|c|}{$\begin{array}{c}\text { Habarra } \\
\text { (Pyrénées atlantiques) }\end{array}$} & \multirow{2}{*}{$\begin{array}{l}\text { Châtillon-Saint-Jean } \\
\text { (Drôme) } \\
\text { (d'après Mourer- } \\
\text { Chauviré, 1972) }\end{array}$} & \multirow{2}{*}{$\begin{array}{c}\text { Mosbach } \\
\text { (Rhénanie) } \\
\\
\text { (d'après Schertz } \\
\text { 1936) } \\
\end{array}$} \\
\hline & $\begin{array}{l}1 \mathrm{AB} \\
\text { femelle } \\
\mathrm{A} 34, \mathrm{dr} .\end{array}$ & \begin{tabular}{|r|}
$1 \mathrm{AB}$ \\
femelle \\
$\mathrm{A} 13, \mathrm{~g}$
\end{tabular} & $\begin{array}{r}2 \mathrm{AB} \\
\text { mâle } \\
\text { A47, d. }\end{array}$ & $\begin{array}{c}2 \mathrm{AB} \\
\text { mâle } \\
\mathrm{B} 35, \mathrm{~g} .\end{array}$ & $\begin{array}{l}\text { Pyrénées } \\
4 \mathrm{E} \\
\text { femelle } \\
\text { E73, dr. }\end{array}$ & $\begin{array}{c}4 \mathrm{E} \\
\text { femelle } \\
\mathrm{E} 36, \mathrm{~g} .\end{array}$ & $\begin{array}{l}\text { 5E } \\
\text { femelle } \\
\text { E16, dr. }\end{array}$ & \begin{tabular}{|c|}
$5 \mathrm{E}$ \\
femelle \\
$\mathrm{E} 13, \mathrm{~g}$.
\end{tabular} & \begin{tabular}{|l} 
7D? \\
femelle \\
D59, dr.
\end{tabular} & \begin{tabular}{|l} 
9D? \\
femelle \\
D72, g.
\end{tabular} & & \\
\hline \multirow{2}{*}{ (1) Longueur } & & 264,0 & & & 285,0 & 284,0 & 278,5 & 278,0 & 276,5 & 280,0 & & $\begin{array}{c}292,8 ; 302,8 \\
\mathrm{n}=2\end{array}$ \\
\hline & & & 290,0 & & & & & & & & $\begin{array}{c}\text { I.V.288-313 } \\
\text { moy }=302,5 ; n=16\end{array}$ & $\begin{array}{l}\text { I.V.302-316,6 } \\
\text { moy. }=303,3 ; n=3\end{array}$ \\
\hline \multirow{2}{*}{$\begin{array}{c}\text { femelles } \\
\text { (2) DT proximal total } \\
\text { máles }\end{array}$} & & 62,0 & & & 64,5 & 64,0 & 61,5 & 60,5 & 59,0 & 66,0 & & $\begin{array}{l}67,6 ; 65,0 \\
\mathrm{n}=2\end{array}$ \\
\hline & & & & & & & & & & & $\begin{array}{c}\text { I.V. } 68,0-74,0 \\
\text { moy. }=70,6 ; \mathrm{n}=16\end{array}$ & $\begin{array}{l}\text { I.V } .72,0-74,7 \\
\text { moy. }=73,6 ; n=3\end{array}$ \\
\hline \multirow{3}{*}{$\begin{array}{l}\text { (3) DT proximal articulaire } \\
\text { (4) DAP proximal articulaire } \\
\text { (5) prticulare }\end{array}$} & 60,5 & 59,0 & 70,5 & 68,5 & 62,5 & 63,5 & 60,0 & 59,5 & 59,0 & 65,5 & & \\
\hline & & 54,5 & 67,0 & & 60,0 & 59,5 & 56,0 & 56,0 & 54,5 & 59,0 & & \\
\hline & & & 80,0 & 80,0 & 69,5 & 69,5 & 68,5 & 68,0 & 67,0 & & & \\
\hline \multirow{2}{*}{$\begin{array}{c}\text { femelles } \\
\text { (6) DT distal articulaire } \\
\text { mâles }\end{array}$} & & & & & 73,5 & 74,0 & 72,5 & 71,5 & 68,5 & & & $\begin{array}{c}80,4 ; 81,8 \\
\mathrm{n}=2\end{array}$ \\
\hline & & & 83,5 & 82,5 & & & & & & & $\begin{array}{l}\text { I.V. } .77,0-86,0 \\
\text { moy. }=80,5: \mathrm{n}=16\end{array}$ & $\begin{array}{l}\text { I.V. } 84,2-88,3 \\
\text { moy. }=85,76 ; n=3\end{array}$ \\
\hline (7) DT minimum diaphyse & 38 & 40,0 & 45,0 & & 36,1 & 37,0 & 37,0 & 36,0 & 34,5 & 39,0 & & \\
\hline \multirow{2}{*}{$\begin{array}{c}\text { femelles } \\
\text { (8) DT au milieu de l'os } \\
\text { mâles }\end{array}$} & & 41,0 & & & & 37,5 & 38,5 & 37,5 & 37,0 & 41 & & $\begin{array}{c}44,3 ; 44,4 \\
n=2\end{array}$ \\
\hline & & & 48,0 & & & & & & & & $\begin{array}{l}\text { I.V. } 46,0-55,6 \\
\text { moy. }=49 ; n=16\end{array}$ & $\begin{array}{l}\text { I.V. } 47,8-48,8 \\
\text { moy. }=48,2 ; n=3\end{array}$ \\
\hline $\begin{array}{l}\text { Indice de robustesse } \\
(7)^{*} 100 /(1)\end{array}$ & & 15,1 & 15,5 & & 12,6 & 13,0 & 13,2 & 12,9 & 12,4 & 13,8 & & \\
\hline \multirow{2}{*}{$\begin{array}{l}\text { femelles } \\
\text { Indice de robustesse: } \\
(8)^{*} 100 /(1) \quad \text { mâles }\end{array}$} & & 15,5 & & & & 13,2 & 13,8 & 13,4 & 13,3 & 14,2 & & $\begin{array}{c}15,1 ; 14,6 \\
\mathrm{n}=2\end{array}$ \\
\hline & & & 16,6 & & & & & & & & $\begin{array}{c}\text { d'après moyennes } \\
\text { moy. }=16,2\end{array}$ & $\begin{array}{c}\text { d'après moyennes } \\
\text { moy. }=15,9\end{array}$ \\
\hline
\end{tabular}

Les dimensions sont données en millimètres ;

I.V. $=$ intervalle de variation $;$ moy $=$ moyenne,$I . C$. moy. $=$ intervalle de confiance de la moyenne

$\mathrm{n}=$ nombre de spécimens ; DAP = diamètre antéro-postérieur ; DT = diamètre transversal ; dr. = droit ; g. = gauche. 
Tableau 35 - Bison priscus Habarra. Premières phalanges postérieures, dimensions. Table 35 - Bison priscus Habarra: Dimensions of the first phalanges of the hindlimb.

\begin{tabular}{|c|c|c|c|c|c|c|c|c|c|c|c|c|}
\hline & \begin{tabular}{|l|}
$1 \mathrm{AB}$ \\
fem. \\
$\mathrm{A} 35$ \\
\end{tabular} & \begin{tabular}{|l}
$1 \mathrm{AB}$ \\
fem. \\
$\mathrm{A} 84$
\end{tabular} & \begin{tabular}{|c}
$1 \mathrm{AB}$ \\
femelle \\
A21 \\
\end{tabular} & $\begin{array}{l}2 \mathrm{AB} \\
\text { mâle } \\
\mathrm{A} 122\end{array}$ & $\begin{array}{l}2 \mathrm{AB} \\
\text { mâle } \\
\text { A119 }\end{array}$ & \begin{tabular}{|l|}
$2 \mathrm{AB}$ \\
mâle \\
$\mathrm{A} 54$
\end{tabular} & \begin{tabular}{|c|}
$2 \mathrm{AB}$ \\
mâle \\
$\mathrm{A} 60$
\end{tabular} & \begin{tabular}{|c|}
$4 \mathrm{E}$ \\
fem. \\
$\mathrm{E} 73 \mathrm{a}$
\end{tabular} & \begin{tabular}{|c|}
$4 \mathrm{E}$ \\
fem. \\
$\mathrm{E} 73 \mathrm{~b}$ \\
\end{tabular} & \begin{tabular}{|c|}
$4 \mathrm{E}$ \\
fem. \\
E37 \\
\end{tabular} & \begin{tabular}{|c|}
$5 \mathrm{E}$ \\
fem. \\
$\mathrm{E} 73$ \\
\end{tabular} & \begin{tabular}{|c|}
$5 \mathrm{E}$ \\
fem. \\
$\mathrm{E} 16$
\end{tabular} \\
\hline (1) L & 77,0 & 76,0 & 77,5 & 84,5 & 86,0 & 86,5 & 85,0 & 79,5 & 79,5 & & & 81,0 \\
\hline (2) D & 38,0 & 37,0 & 38,5 & 44,0 & 42,5 & 43,5 & & 38,0 & 37,0 & 37,0 & 37,0 & 37,0 \\
\hline & 41,5 & 40,5 & 42,5 & 48,0 & 48,5 & 49,0 & & 43,5 & 43,5 & 38,5 & 43,0 & 40,5 \\
\hline ticulai & 36,0 & 34,5 & 35,0 & 40,5 & 41,5 & 41,5 & 42,0 & 36,5 & 34,0 & & & 33,5 \\
\hline $\begin{array}{l}\text { (5) DAP distal } \\
\end{array}$ & 26,5 & 25,5 & 25,5 & 30,5 & 30,0 & 30,0 & 30,0 & 26,5 & 26,0 & & & 27,0 \\
\hline (6) DT minimum de la & 33,5 & 32,0 & 32,5 & 37,0 & 37,5 & 38,0 & 38,0 & 32,5 & 31,5 & & & 30,5 \\
\hline
\end{tabular}

Les dimensions sont données en millimètres ;

Pour l'emplacement des diamètres mesurés, voir figure 8

$\mathrm{DAP}=$ diamètre antéro-postérieur ; DT = diamètre transversal

Tableau 36 - Bison priscus Habarra. Deuxièmes phalanges postérieures, dimensions.

Table 36 - Bison priscus Habarra: Dimensions of the second phalanges of the hindlimb.

\begin{tabular}{|c|c|c|c|c|c|c|c|c|}
\hline & $\begin{array}{l}1 \mathrm{AB} \\
\text { fem. } \\
\mathrm{A} 30\end{array}$ & $\begin{array}{c}2 \mathrm{AB} \\
\text { mâle } \\
\mathrm{A} 95\end{array}$ & $\begin{array}{l}2 \mathrm{AB} \\
\text { mâle } \\
\mathrm{A} 120\end{array}$ & $\begin{array}{c}2 \mathrm{AB} \\
\text { mâle } \\
\mathrm{A} 87\end{array}$ & $\begin{array}{l}2 \mathrm{AB} \\
\text { mâle } \\
\mathrm{A} 121\end{array}$ & $\begin{array}{c}4 \mathrm{E} \\
\text { fem. } \\
\text { E73a }\end{array}$ & $\begin{array}{c}4 \mathrm{E} \\
\text { fem. } \\
\text { E73b }\end{array}$ & $\begin{array}{c}5 \mathrm{E} \\
\text { fem. } \\
\mathrm{E} 16\end{array}$ \\
\hline (1) Longueur totale & 52,0 & 58,0 & 57,5 & 57,5 & 58,0 & 55,5 & 55,5 & 57,5 \\
\hline (2) DT proximal total & 37,0 & 44,5 & 43,5 & 45,0 & 45,0 & 40,0 & 39,5 & 39,0 \\
\hline (3) DT proximal articulaire & 35,5 & 41,5 & 40,0 & 41,0 & 43,0 & 37,0 & 37,0 & 36,0 \\
\hline (4) DAP proximal & 36,5 & 43,5 & 43,0 & 43,5 & 45,0 & 40,0 & 39,5 & 39,0 \\
\hline (5) DT distal & 31,0 & 38,0 & 37,5 & 38,0 & 38,0 & 32,0 & 31,5 & 30,5 \\
\hline (6) DAP distal & 34,0 & 39,5 & 38,0 & 38,0 & 39,5 & 36,0 & 34,5 & 36,0 \\
\hline (7) DT de la région moyenne & 28,5 & 34,5 & 32,5 & 32,0 & 34,5 & 30,5 & 28,5 & 28,5 \\
\hline (8) DAP minimum de la région 1 & 27,0 & 33,0 & 30,5 & 30,5 & 33,0 & 30,5 & 27,5 & 29,0 \\
\hline
\end{tabular}

Les dimensions sont données en millimètres ;

Pour l'emplacement des diamètres mesurés, voir figure $8 \mathrm{H}$

$\mathrm{DAP}=$ diamètre antéro-postérieur ; DT = diamètre transversal

Tableau 37 - Bison priscus Habarra. Troisièmes phalanges antérieures et postérieures, dimensions. Table 37 - Bison priscus Habarra: Dimensions of the third phalanges of the fore- and hind-limbs.

\begin{tabular}{|l|c|c|c|c|c|}
\cline { 2 - 6 } \multicolumn{1}{c|}{} & \multicolumn{3}{c|}{ antérieures } & \multicolumn{2}{c|}{ postérieures } \\
\cline { 2 - 6 } \multicolumn{1}{c|}{} & \begin{tabular}{c}
$2 \mathrm{AB}$ \\
mâle \\
\cline { 2 - 5 } \multicolumn{1}{c|}{}
\end{tabular} & $\begin{array}{c}2 \mathrm{AB} \\
\text { mâle } \\
\mathrm{A} 96\end{array}$ & $\begin{array}{c}? \\
\mathrm{~B} 65\end{array}$ & $\begin{array}{c}2 \mathrm{AB} \\
\text { mâle } \\
\text { N1 }\end{array}$ & $\begin{array}{c}5 \mathrm{E} \\
\text { femelle } \\
\text { E21 }\end{array}$ \\
\hline $\begin{array}{l}\text { Face latérale } \\
\text { (1) Diamètre antéro-postérieur dorsal } \\
\text { (H) Hauteur }\end{array}$ & 109 & 107,5 & 102,5 & 94 env. & 78,5 \\
\hline Face palmaire ou face plantaire & 54,5 & 54,5 & 49,5 & 56 & 43 \\
\hline $\begin{array}{l}\text { (3) Diamètre antéro-postérieur } \\
\text { (2) Diamètre transversal }\end{array}$ & 78 & 79 env. & 76,5 & 77 env. & 61 \\
\hline
\end{tabular}

Les dimensions sont données en millimètres ;

Pour l'emplacement des diamètres mesurés, voir figure $5 \mathrm{D}$ et $\mathrm{E}$ 
Tableau 38 - Bison priscus Habarra. Sésamoïdes abaxiaux et axiaux, dimensions. Table 38 - Bison priscus Habarra abaxial and axial sesamoid dimensions.

\begin{tabular}{|l|c|c|c|c|}
\cline { 2 - 5 } \multicolumn{1}{c|}{} & \multicolumn{2}{c|}{ abaxiaux } & \multicolumn{2}{c|}{ axiaux } \\
\cline { 2 - 5 } \multicolumn{1}{c|}{} & $2 \mathrm{AB}$ & $4 \mathrm{E}$ & $2 \mathrm{AB}$ & $4 \mathrm{E}$ \\
& mâle & femelle & mâle & femelle \\
\cline { 2 - 5 } & $\mathrm{A} 86$ & $\mathrm{E} 37$ & $\mathrm{~A} 57$ & E37 \\
\hline Diamètre proximo-distal total & 36,5 & & 33,0 & 28,0 \\
\hline Diamètre proximo-distal articulaire maximum & 27,0 & 23,0 & 29,5 & 26,0 \\
\hline Diamètre transversal total & 25,5 & 19,0 & 21,5 & 17,5 \\
\hline Diamètre transversal articulaire maximum & 24,0 & 18,5 & 20,5 & 17,0 \\
\hline
\end{tabular}

Les dimensions sont données en millimètres

Tableau 39 - Bison priscus Habarra. Atlas et axis, dimensions. Table 39 - Bison priscus Habarra axis and atlas dimensions.

\begin{tabular}{|c|c|c|c|}
\hline & $\begin{array}{c}1 \mathrm{AB} \\
\text { femelle }\end{array}$ & \multicolumn{2}{|c|}{$\begin{array}{c}4 \mathrm{E} \\
\text { femelle }\end{array}$} \\
\hline ATLAS & A19 & E70 & \\
\hline Diamètre transversal total (fig. 23-1a) & 166 & 166,5 & \\
\hline Diamètre crânio-caudal total (fig. 23-1b) & & 120,0 & \\
\hline Diamètre crânio-caudal de la face articulaire crâniale & & & \\
\hline à la face articulaire caudale suivant le plan sagittal & 102 & 105,5 & \\
\hline Diamètre transversal maximum de la face & & & \\
\hline articulaire crâniale (fig. 23-2) & 126 & 127 & \\
\hline Diamètre transversal maximum de la face & & & \\
\hline articulaire caudale (fig. 23-3) & 115 & 115 & \\
\hline En vue dorsale : distance minimum entre les & 80 & 835 & \\
\hline $\begin{array}{l}\text { foramens alaıres } \\
\text { En vue crâniale : largeur maximum du trou vertébral }\end{array}$ & 80 & 83,5 & \\
\hline perpendiculairement au plan sagittal & $55 ?$ & 50 & \\
\hline $\begin{array}{l}\text { En vue caudale : largeur maximum du trou vertébral } \\
\text { perpendiculairement au plan sagittal }\end{array}$ & & 64 & \\
\hline AXIS & & & E75 \\
\hline Longueur (fig. 23-4) & & & 120 \\
\hline $\begin{array}{l}\text { Diamètre transversal de la face articulaire crâniale } \\
\text { (fig. 23-5b) }\end{array}$ & & & 112 \\
\hline Diamètre transversal de la face articulaire caudale & & & $57 ?$ \\
\hline $\begin{array}{l}\text { Diamètre transversal au niveau des processus } \\
\text { articulaire caudaux }\end{array}$ & & & 82 \\
\hline En vue ventrale, diamètre transversal minimum & & & 67 \\
\hline $\begin{array}{l}\text { Diamètre transversal maximum du processus } \\
\text { odontoïde (fig. 23-5a) }\end{array}$ & & & 59 \\
\hline
\end{tabular}

Les dimensions sont données en millimètres. Le degré de précision de la mesure est satisfaisant si la valeur comporte un chiffre après la virgule (ex. 166,5), est relativement satisfaisant si il n'y a pas de virgule après l'unité ( ex. 115), est approximatif si la valeur est suivie d'un point d'interrogation (ex. 57?) 
Tableau 40 - Bison priscus Habarra, femelle $n^{\circ} 4 \mathrm{E}$. Vertèbres cervicales, thoraciques et lombaires ; dimensions.

Table 40 - Bison priscus Habarra female 4E : Cervical, thoracic, and lumbar vertebrae dimensions.

\begin{tabular}{|c|c|c|c|c|c|c|c|c|c|c|c|c|c|c|c|}
\hline & \begin{tabular}{|c|} 
C3 \\
E73
\end{tabular} & \begin{tabular}{|c|} 
C4 \\
E73
\end{tabular} & $\begin{array}{c}\text { C5 } \\
\text { E56 }\end{array}$ & \begin{tabular}{|c|}
$\mathrm{C} 6$ \\
$\mathrm{E} 32$ \\
\end{tabular} & \begin{tabular}{c|c|} 
C7 \\
E31
\end{tabular} & \begin{tabular}{|c|} 
T1 \\
E30
\end{tabular} & $\begin{array}{l}\mathrm{T} 2 \\
\mathrm{E} 7\end{array}$ & \begin{tabular}{|c|} 
T8ouT9 \\
E44
\end{tabular} & \begin{tabular}{|c|} 
T9ouT10 \\
E34
\end{tabular} & \begin{tabular}{|l|} 
T12 \\
E43
\end{tabular} & \begin{tabular}{|l|} 
T13 \\
E35 \\
\end{tabular} & \begin{tabular}{|c|} 
L1 \\
E50
\end{tabular} & \begin{tabular}{|c|} 
L2 \\
E41 \\
\end{tabular} & \begin{tabular}{|c|} 
L4 \\
E40 \\
\end{tabular} & $\begin{array}{c}\text { L5 } \\
\text { E65 }\end{array}$ \\
\hline Longueur du corps (fig. 22-1) & 90,0 & 88,0 & 85,5 & 83,5 & 70,0 & 74,5 & 68,5 & 62 & 61,5 & 65,5 & 66,0 & 74,0 & 75,5 & 79,5 & 73,0 \\
\hline $\begin{array}{l}\text { Longueur du canal (fig. } 22-2 \text { et } 3 \text { : distance } \\
\text { entre A et B) }\end{array}$ & 67,0 & 60,0 & 58,0 & 57,0 & 53,0 & 57,0 & 59,0 & 57,5 & 58,0 & 60,0 & 62,5 & 67,5 & 70,0 & $71 ?$ & 67,0 \\
\hline $\begin{array}{l}\text { Diamètre transversal de la tête perpendicu- } \\
\text { lairement au plan axial (fig. 23-6b) }\end{array}$ & 38,5 & 37,5 & 36,5 & 36,0 & 38,5 & 42,0 & 42,0 & & & & & \begin{tabular}{|l|}
49,0 \\
\end{tabular} & 49,0 & 55,0 & 61,5 \\
\hline $\begin{array}{l}\text { Diamètre dorso-ventral de la tête suivant le } \\
\text { plan axial (fig. 22-1) }\end{array}$ & 51,5 & 49 & 49 & 53,5 & 55,5 & 54,5 & 52,0 & & & & & 44,5 & 45 & 48,5 & 46,5 \\
\hline $\begin{array}{l}\text { Diamètre transversal maximum de la fosse } \\
\text { perpendiculairement au plan axial }\end{array}$ & 58,5 & $59 ?$ & 58,5 & 53,0 & 52,5 & 47,0 & 43,0 & & & & & \begin{tabular}{|l|}
56,0 \\
\end{tabular} & 56,5 & 70,5 & 87,5 \\
\hline $\begin{array}{l}\text { Diamètre dorso-ventral de la fosse } \\
\text { (maximum) dans le plan axial }\end{array}$ & 55,5 & & & 56,0 & 50 & 48,5 & & & & & & \begin{tabular}{|l|}
43,0 \\
\end{tabular} & 45,0 & 42,5 & 38,0 \\
\hline $\begin{array}{l}\text { Diamètre transverse maximum de la vertèbre au } \\
\text { niveau des processus transverses (fig. } 22-2 \mathrm{c} \text { ) }\end{array}$ & & & & & & 46,5 & & & & & & & & & \\
\hline $\begin{array}{l}\text { Diamètre transversal au-dessus des processus } \\
\text { transverses (fig. } 23-6 \mathrm{a}-\mathrm{a} \text { ) }\end{array}$ & 53,0 & 66,0 & 72,5 & 77,0 & 80,5 & 114 & & & & & & & & & \\
\hline $\begin{array}{l}\text { Diamètre crânio-caudal au niveau des } \\
\text { processus articulaires }\end{array}$ & 95,5 & & $84 ?$ & 84,5 & & & & & & & & & & & \\
\hline $\begin{array}{l}\text { Diamètre transversal maximum au niveau des } \\
\text { processus articulaires crâniaux }\end{array}$ & & & & & & & & & & & & & 69,0 & & 98,5 \\
\hline $\begin{array}{l}\text { Diamètre transversal entre les processus } \\
\text { articulaires crâniaux et les processus transverses }\end{array}$ & & & & & & & & & & & & & 62,5 & 71 & 91,5 \\
\hline $\begin{array}{l}\text { Diamètre transversal maximum au niveau des } \\
\text { processus articulaires caudaux }\end{array}$ & & & & & & & & & & & & & 46,0 & 68,0 & $76 ?$ \\
\hline $\begin{array}{l}\text { Diamètre transversal entre les processus } \\
\text { articulaires caudaux et les processus transverses }\end{array}$ & & & & & & & & & & & & & 55,5 & 72,0 & 77,5 \\
\hline
\end{tabular}

Tableau 41 - Genre Bison. Sacrum, dimensions.

Table 41 - Bison sacrum dimensions.

Longueur totale (fig. 22-4)

Longueur de l'épine sacrale -fig. 22-5a)

Longueur maximum : de la tête de la première vertèbre sacrée

à la fosse de la dernière

Diamètre transversal de la tête de la $1^{\text {ére }}$ vertèbre sacrée

Diamètre dorso-ventral de la tête de la $1^{\text {ère }}$ vertèbre sacrée

Hauteur du processus de la $1^{\text {ère }}$ vertèbre sacrée à partir de la voûte du canal

Largeur maximum de l'épine sacrale (au niveau de la $2^{\text {ème }}$

vertèbre sacrée (fig. 22-5b)

\begin{tabular}{|c|c|}
\hline $\begin{array}{l}\text { Bison priscus } \\
\text { femelle }\end{array}$ & $\begin{array}{l}\text { Bison } \\
\text { bison }\end{array}$ \\
\hline Habarra $n^{\circ} 4 \mathrm{E}$ & IPGQ \\
\hline E15 & $\begin{array}{c}\text { KU. } \\
23002\end{array}$ \\
\hline 274 & 248 \\
\hline 234 & 216,5 \\
\hline 261 & 244,5 \\
\hline 95 & 72 \\
\hline 41 & 37 \\
\hline 71 & 90 \\
\hline 23 & 29 \\
\hline
\end{tabular}

N.B. : sur la pièce fossile l'épine sacrale est nettement moins haute que sur le sacrum, pourtant plus petit, du bison actuel.

Les dimensions sont données en millimètres. Le degré de précision de la mesure est satisfaisant si la valeur comporte un chiffre après la virgule (ex. 244,5), est relativement satisfaisant si il n'y a pas de virgule après l'unité ( ex. 71). 
Tableau 42 - Liste, références, latéralisation et localisation par secteur des vestiges rapportés aux 18 bisons identifiés dans la grotte d'Habarra.

Table 42 - Specimen list by body part, side, and sector location for the 18 Habarra Cave bison.

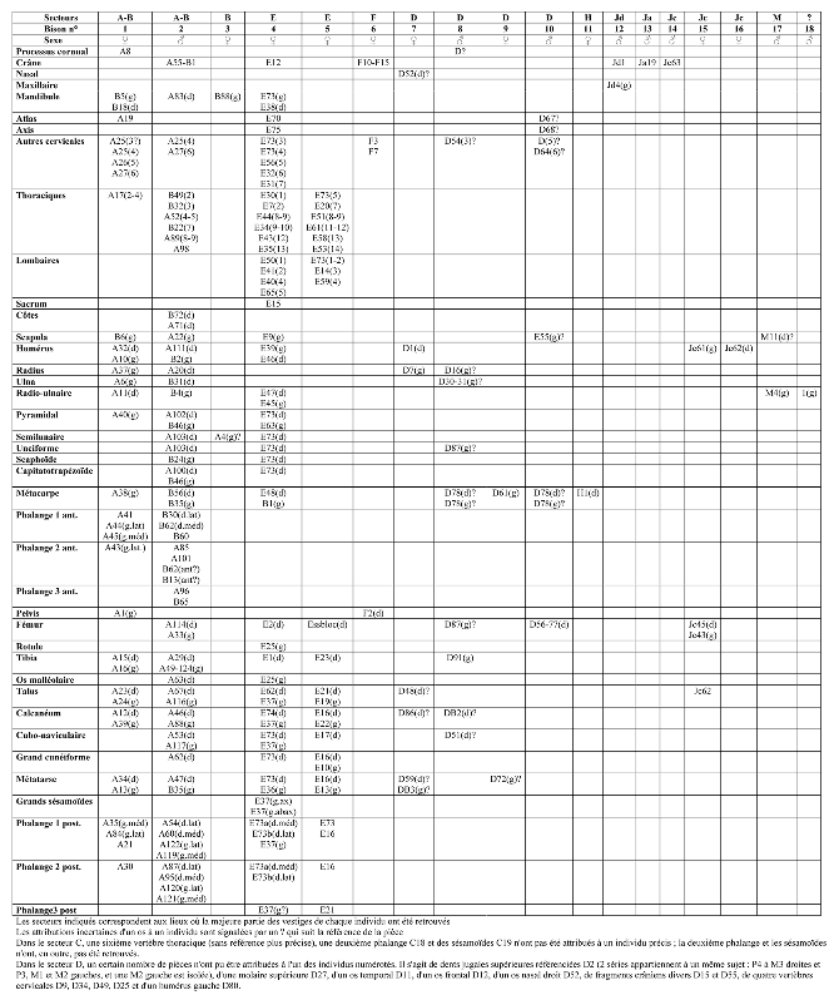

\section{BIBLIOGRAPHIE}

BARONE R. 1986 - Anatomie comparée des animaux domestiques. Tome 1 : Ostéologie. Paris. Vigot. 761 p. $423 \mathrm{pl}$.

BEDFORD J.N. 1978 - A technique for determination of nature bison metapodials. Plains Anthrop., 23, p. 40-43.

BIBIKOVA V.I. 1958 - Some distinguishing features in the bones of the genera Bison and Bos (en russe). Bull. Mosk. Obschschestwa Isp. Priroda. 63/6, p. 23-35.

BORDES Fr. et LABROT J., 1967 - La stratigraphie du gisement de Roc de Combe (Lot) et ses implications. Bulletin de la Société Préhistorique Française, LXIV, n 1, p. 15-28.

BREUIL H. 1950 - Les peintures et gravures de la caverne de Niaux. Bulletin de la Société préhistorique de l'Ariège, t. 5, p. 9-34, 22 fig., 2 pl. h.t.

BREUIL H. 1952 - La caverne de Niaux. Compléments inédits sur la décoration. Bulletin de la Société préhistorique de l'Ariège, t. 7, p. 1-35, 27 fig., 4 pl. h.t. 
BRUGAL J.-Ph., 1983 - Application des analyses multidimensionnelles à l'étude du squelette des membres des grands Bovidés pléistocènes (grotte de Lunel-Viel, Hérault : perspectives évolutives. Thèse de 3ème cycle, Géologie, Université d'Aix-Marseille II, 449 p., 97 fig., 40 tabl.

BYRNE N. 1979 - Le gisement de La Roche-Cotard à Langeais : Bovidae. In J.-Cl. Marquet (Ed.) : La Roche Cotard à Langeais, Université François Rabelais, Tours, p. 64-79, 1 fig., 1 tabl., 2 pl.

CLOT A. et DURANTHON Fr. 1990 - Les mammifères fossiles du Quaternaire dans les Pyrénées. Muséum d'Histoire naturelle de Toulouse, Accord Edit., 159 p., 90 fig., 21 tabl.

Collectif, 1969 - La France au temps des mammouths. Hachette, Coll. Ages d'Or et Réalités, 256 p., 247 fig.

Collectif, 1980 - Spéléologie et Préhistoire béarnaises. La grotte des Bisons à Lurbe-Saint-Christau. Catalogue de l'exposition, Musée béarnais, château de Pau, 27 mars-24 avril 1980. Groupe archéologique des Pyrénées Occidentales éd. 8 p.

CROUZEL F., MANENC A. et REVEL J-C. 1982 - Squelettes de Bison priscus Bojanus ssp gigas Flerov 1969 dans une grotte ariégeoise. Bulletin de la Société d'Histoire naturelle de Toulouse, 118, p. 71-100, 9 fig.

DEGERBÖL M. et IVERSEN J. 1945 - The bison of Denmark. Geoloske Undesogelse, 73, p. 1-62, 13 fig., 3 pl., 2 tabl.

DELPECH Fr. 1972 - Fouilles de sauvetage dans le gisement magdalénien de Fongaban, commune de Saint-Emilion (Gironde). La faune. L'Anthropologie, t. 76, p. 615-629, 2 fig., 10 tabl.

DELPECH Fr. 1984 - La Ferrassie. Carnivores, Artiodactyles et Périssodactyles. In Henri Delporte (Ed.) : Le grand abri de La Ferrassie. Fouilles 1968-1973. Etudes Quaternaires, I.P.H. Paris, mémoire 7, p. 61-89, 5 fig., 27 tabl., 4 pl.

DELPECH Fr. 1999a - Biomasse d'Ongulés au paléolithique et inférences démographiques. Paléo, 11, p. 19-42, 10 fig., 4 tabl.

DELPECH Fr. 1999b - La chasse au Bison dans le Sud-Ouest de la France au cours du Würm : Choix humain ou contraintes paléoenvironnementales ? : In J.-Ph. Brugal, Fr. David, J. G. Enloe ; J. Jaubert (eds) : Le Bison : gibier et moyen de subsistance des hommes du Paléolithique aux Paléoindiens des grandes plaines, Actes du Colloque International, Toulouse 1995, Editions APDCA, Antibes, 1999, p. 63-84, 12 fig., 4 tabl.

DELPECH Fr. 2003 - L'environnement animal des européens au Paléolithique supérieur In R. Desbrosse et A. Thévenin (eds.) : Préhistoire de l'Europe. Des origines à l'Age du Bronze, Actes des congrès nationaux des sociétés historiques et scientifiques, 125è Lille, 2000, Editions du C.T.H.S., p. 271-289, 4 fig., 2 tabl.

DELPECH Fr. et LENOIR M. 1996 - Eléments d'interprétation sur l'occupation magdalénienne de la bordure nord du Massif pyrénéen. In H. Delporte et J. Clottes (eds) : Pyrénées préhistoriques, arts et sociétés, Actes du 118e Congrès National des Sociétés Savantes, Pau 1993, Editions du C.T.H.S., p. 217-224, 3 tabl.

DRIESCH Ang. von den 1976 - A guide to the measurement of animals bones from archaeological sites. Peabody Museum, Harvard University Cambridge (U.S.A.), bull. n 1, 136 p., 62 fig.

FULLER W A 1959 - The horns and teeth as indicators of age in bison. I : Wildlife Manadgment., 23, p. 342-344. 
GUADELLI J.-L. 1987 - Contribution à l'étude des zoocoenoses préhistoriques en Aquitaine (Würm ancien et interstade würmien). Thèse de Doctorat de 3ème cycle, Université Bordeaux I, texte : 568 p., 163 fig., 424 tabl.

GUÉRIN Cl. et PATOU-MATHIS M. (eds), 1996 - Les grands mammifêres plio-pléistocènes d'Europe. Masson, collection Préhistoire, 292 p., 73 fig.

GUTHRIE R. D. 1990 - Frozen Fauna of the Mammoth Steppe. The story of Blue Babe. The University of Chicago Press, Chicago and London, 323 p., 178 fig., 8 tabl.

HAYNES G. 1984 - Tooth wear in northern bison. Journal of Mammalogy, 65, p. 487-490.

KOCH W. 1934 - The age order of the European bison (Bos bonasus). Anat. Record, 61, p. 371-376.

KOWALSKI K. 1967 - The evolution and fossil remains of the european bison. ActaTheriologica, vol. XII, 21, p. 335-338.

LEHMAN U. von 1949 - Der Ur im Diluvium Deutchlands und seine Verbreitung. Neues Jahrb. Min. Geol. und Paleont., Abt. 90/2, p. 163-266. Stuttgart.

MCCUAIG BALKWILL D. et CUMBAA S. L. 1992 - A guide to the identification of postcranial bones of Bos taurus and Bison bison. Syllogeus 71. Canadian Museum of Nature, ottawa, Canada K1 P6 P4, 277 p., 96 fig.

McDONALD J. N. 1981 - North American bison: their classification and evolution. Berkeley, University of California Press, 316 p., 104 fig., 75 tabl.

MARSAN G. 1985 - Rapport de fouilles de sauvetage (1984) à Arudy (Pyrénées Atlantiques), lieudit Habarra. Document interne au S.R.A. Midi-Pyrénées.

MASSAUD J. 1984 - Le Bos primigenius de la vallée des Eaux-Claires (Torsac, Charente). Bulletin de la Société de Spéléologie et de Préhistoire de Bordeaux, t. XXV, 72 p., 4 fig., 28 tabl., 12 pl.

MILLOT J. 1945 - Les bisons européens des collections du Muséum d'Histoire Naturelle. Mammalia, vol. 9, n 10, p. 1-19, 4 tabl., 4 fig.

MOURER-CHAUVIRÉ C. 1972 - Etude de nouveaux restes de vertébrés provenant de la carrière Fournier à Châtillon-Saint-Jean (Drôme). III : Artiodactyles, Chevaux, Oiseaux. Bulletin de l'Association Française d'Etude du Quaternaire, Paris, t. 33, n 4, p. 271-305, 3 pl.

OLSEN St. J. 1960 - Postcranial skeletal characters of Bison and Bos. Papers of the Peabody Museum, Harvard University, t. XXXV, nº 4, 15 p., 24 fig.

PALES L. 1981 - Les gravures de La Marche, III : Equidés et Bovidés. Ophrys, 150 p., 52 fig., 124 pl. POPLIN Fr. 1984 - Sur le profil dorso-lombaire des bisons dans l'art paléolithique. In H.G. Bandi, W. Huber, M.R. Sauter et B. Sitter (éds) : La contribution de la zoologie et de l'éthologie à l'interprétation de l'art des peuples chasseurs préhistoriques. Editions universitaires, Fribourg, Suisse, p. 217-242.

PRAT Fr. 1968 - Recherches sur les Equidés pléistocènes en France. Thèse de doctorat d'Etat ès Sciences Naturelles nº 226, Faculté des Sciences de Bordeaux, 696 p. de texte, 149 fig., 126 tabl. REHER C. A. 1974 - Population of the Casper Site bison. In Georges C. Frison (ed.): The Casper Site, New-York: Academic Press, p. 113-124.

RESHETOV V. Y. 1974 - Etude comparative ostéologique du squelette post-crânien des bisons en relation avec l'histoire du genre Bison (en russe). Teriologija, 2, p. 57-64.

RIGAUD J.-Ph. 1986 - Gouffre d'Habarra. Circonscription d'Aquitaine. Gallia Préhistoire, t. 29, 2, p. 253-254. 
ROSKOSZ T. et EMPEL W. 1961 - The size of the head and the height of the spinous processes in the region of the withers of the European bison. Acta Theriologica, vol. 6, p. 63-69, 4 tabl.

SCHERTZ E. 1936a - Zur Untersscheidung von Bison priscus Boj. und Bos primigenius Boj. an Metapodien und Astragalus, nebst Bemerkungen über einige diluviale Fundstellen. Senckenbergiana, 18/1-6, p. 37-71.

SCHERTZ E. 1936b - Der Geschlets-Unterschied an metapodien von Bison. Senckenbergiana, 18/1-6, p. 357-381.

SIMPSON G. G. 1941 - Large Pleistocene Felines of North America. American Museum Novitates, 1136, p. 1-27.

SIMPSON G. G., Roe A. et Lewontin R. C. 1960 - Quantitative Zoology. Harcourt, Bruce and Compagny. New York Burlingam

Skinner M. F. et Kaisen O. C. 1947 - The fossil bison of Alaska and preliminary revision of the genus. Bulletin of American Museum of Natural History, 89, p. 126-256, 5 fig., 25 tabl., 26 pl., 3 cartes.

SLOTT-MOLLER R. 1988 - Contribution à l'étude paléontologique d'un gisement préhistorique : l'exemple du Roc de Marcamps (Gironde). Diplôme d'études supérieures de Sciences naturelles, Université Bordeaux I, 173 p., 146 tabl., 70 pl.

SLOTT-MOLLER R. 1990 - La faune de La Borde. In J. Jaubert, M. Lorblanchet, H. Laville, R. SlottMoller, A. Turq et J.-Ph. Brugal (eds) : Les chasseurs d'Aurochs de La Borde. D.A.F. éditions des Sciences de l'Homme, Paris, p. 33-68, fig. 23-47, 35 tabl.

SONNEVILLE-BORDES D. de 1967 - La Préhistoire moderne. P. Fanlac, Périgueux, 146 p., 46 fig., 140 ill.

STAMPFLI H. R. 1963 - Wisent, Ur und Hausrind. In J. Boessneck, J.P. Jéquier et H.R. Stampfli (eds) : Seeberg, burgäschisee Sud. Die Tierreste. Acta Bernensia, 2, fasc. 3, Berne, 215 p., 42 fig., XXIII pl., 17 tabl.

TOURNEPICHE J.-Fr. 1991 - Découverte d'un Bison priscus Boj., 1827 à Sainte-Croix de Mareuil (Dordogne). Paléo, 3, p. 93-100, 13 fig.

\section{NOTES}

1. Toutes les datations ont été effectuées sur ossements suivant la méthode classique $d u{ }^{14} \mathrm{C}$. Pour le Cheval, il s'agissait d'un troisième métatarsien et de cinq fragments de côtes provenant du carré $\mathrm{K}$, secteur $\mathrm{Kb}$, qui appartenaient vraisemblablement à un même individu ; pour le Cerf, ce sont des ossements de même sujet fossilisés dans le carré $\mathrm{L}$; pour le Bison, il s'agit d'un radius gauche complet du carré $\mathrm{E}$; on verra qu'il est attribuable à la femelle qui porte le $\mathrm{n}^{\circ} 4$ (cf. infra).

2. Nous avons écrit le mot bison (bisons) avec une minuscule quand il désigne un individu ou des individus (ex. les ossements du bison $n^{\circ} 2$ ), avec une majuscule quand il désigne le genre (ex. les ossements de Bison abondent).

3. Ce sont des fragments osseux non déterminés trouvés en association avec le crâne qui ont été datés suivant la méthode classique $\mathrm{du}^{14} \mathrm{C}$.

4. Ce sont des fragments osseux non déterminés trouvés en association avec le crâne qui ont été datés suivant la méthode classique $\mathrm{du}^{14} \mathrm{C}$. 


\section{RÉSUMÉS}

Au cours du Quaternaire, vraisemblablement à plusieurs reprises, la grotte d'Habarra a piégé de nombreux grands mammifères passés par des deux ouvertures naturelles du toit de la caverne. Le gisement paléontologique a été découvert en 1984 et des fouilles furent pratiquées la même année réunissant des membres du Groupe Spéléologique de la Vallée d'Ossau (G.S.V.O.), du Groupe Archéologique des Pyrénées-Occidentales (G.A.P.O), de la Direction des Antiquités Préhistoriques d'Aquitaine et de l'Institut du Quaternaire de l'Université de Bordeaux I. Bien que le remplissage fossilifère n'ait été que faiblement entamé, de nombreux ossements furent exhumés se rapportant à plusieurs taxons de grands mammifères pléistocènes : Renne, Cheval, Cerf, Rhinocéros... mais le taxon le mieux représenté est le Bison des steppes tant en nombre de restes qu'en nombre d'individus. Vingt bisons au moins sont représentés et quatre d'entre eux le sont par un nombre élevé d'éléments de leurs squelettes. L'ensemble constitue une documentation sur Bison priscus exceptionnelle qui a été exploitée dans des directions qui ne peuvent être abordées qu'à partir d'échantillons importants.

Des éléments de morphologie et de biométrie ont été recherchés pour une distinction 1) au niveau sexuel : pour Bison priscus de nombreux caractères distinctifs mâle-femelle ont été établis ; 2) au niveau spécifique: quelques points concernant notamment les dimensions relatives de grands segments osseux ont pu être précisés ; ils aident à caractériser Bison priscus ; relativement à Bison bison et Bison bonasus ; 3 ) au niveau générique : l'évidence de l'appartenance des ossements de Bovinés d'Habarra à Bison priscus a permis d'aborder la question de la pertinence de certains critères de différenciation entre les deux genres Bos et Bison.

Ce travail a été conçu afin d'en rendre aisée sa consultation, un peu comme un manuel d'anatomie comparée propre aux grands Bovidés qui serait fondé non seulement sur des données actuelles mais aussi sur des restes fossiles : la table des matières, détaillée, permet de localiser rapidement l'emplacement des descriptions et informations relatives soit à un os soit à un individu particulier et le tableau 42 donne pour chaque individu la liste des os lui appartenant.

Tout en sachant qu'il n'est pas sans défauts, nous souhaitons que ce travail soit utile et contribue efficacement à faire avancer les connaissances sur ce grand ongulé pléistocène qu'était le Bison des steppes; en effet, si la silhouette de Bison priscus nous est familière grâce aux représentations que nous en ont laissées les peintres et graveurs préhistoriques, son squelette était jusqu'à présent assez mal documenté, du moins dans sa variabilité morphologique et biométrique.

During the Quaternary, Habarra cave acted as a natural trap: many large mammals fell through two natural openings in the roof and became entombed within it. Discovered in 1984, the site was subsequently excavated by members of the Groupe Spéléologique de la Vallée d'Ossau (G.S.V.O.), the Groupe Archéologique des Pyrénées-Occidentales (G.A.P.O), the Direction des Antiquités Préhistoriques d'Aquitaine, and the Institut du Quaternaire of the Université de Bordeaux I. Even though the excavations were not extensive, many fossil specimens from a variety of large Pleistocene mammals were retrieved, including those of reindeer, horse, red deer, and rhinoceros. Best documented in terms of both specimens and individuals is the steppe bison (Bison priscus). The remains of at least 20 of these animals were collected, of which four individuals are particularly well-represented. The collection as a whole provides remarkably thorough documentation of the Bison priscus skeleton, in turn allowing detailed osteological analyses.

We have used morphological and biometric analyses to improve our understanding of 1) sexual differences within Bison priscus, establishing a number of skeletal characters that can be used to distinguish males and females of this species; 2) species-level differences, providing biometric 
data that allow more secure differentiation of Bison priscus from Bison bison and Bison bonasus, and, 3) genus-level differences, since we have been able to use the Habarra Cave Bison priscus material to examine the validity of a series of criteria that may allow the differentiation of Bos and Bison. We have presented our results in the form of a manual of comparative anatomy for Bos and Bison, a manual founded on both modern material and on the fossil remains of the animals involved. The detailed table of contents will allow the reader to quickly find descriptions and information relevant to a particular skeletal element or individual, while Table 42 provides a list of specimens for each Habarra Bison priscus individual. The profile of Bison priscus is familiar to us from the depictions left by prehistoric artists, but much less has been known of the skeleton of this remarkable Pleistocene ungulate, at least in terms of morphological and biometric variability. Although we are fully aware that this work is not without fault, it is our hope that it will help fill this significant gap in our knowledge.

\section{INDEX}

Keywords : Bison priscus, Bovinae, paleontology, comparative anatomy, morphological and biometrical variability

Mots-clés : Bison priscus, Bovinae, paléontologie, anatomie comparée, variabilité morphologique et biométrique

\section{AUTEURS}

\section{FRANÇOIS PRAT}

Professeur honoraire de l'Université Bordeaux I, Institut de Préhistoire et Géologie du Quaternaire, 33405 Talence Cedex et rue Fanning Lafontaine, 33400 Pessac.

\section{FRANÇOISE DELPECH}

Université Bordeaux I, Institut de Préhistoire et de Géologie du Quaternaire, UMR 5808 du CNRS, Avenue des Facultés, 33405 Talence Cedex.

\section{NICOLAS CANCEL}

Avenue de la République 17770 Burie.

\section{JEAN-LUC GUADELLI}

Université Bordeaux I, Institut de Préhistoire et de Géologie du Quaternaire, UMR 5808 du CNRS, Avenue des Facultés, 33405 Talence Cedex.

\section{RENÉ SLOTT-MOLLER}

Domaine de Camblanne, 33710 Saint-Seurin de Bourg. 